\title{
ANALYSIS OF DELAYS IN NETWORKED \\ FLIGHT SIMULATORS
}

\author{
By \\ MOHAMMAD B. MENHAJ \\ Bachelor of Science \\ Melli University \\ Tehran, Iran \\ 1980 \\ Master of Science \\ Oklahoma State University \\ Oklahoma, stillwater \\ 1988
}

Submitted to the Faculty of the

Graduate College of the

Oklahoma State University

in partial fulfillment of

the requirements for

the Degree of

DOCTOR OF PHILOSOPHY

May, 1992 


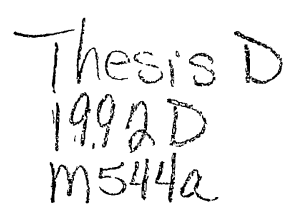




\section{ANALYSIS OF DELAYS IN NETWORKED}

\section{FLIGHT SIMULATORS}

Thesis Approved:

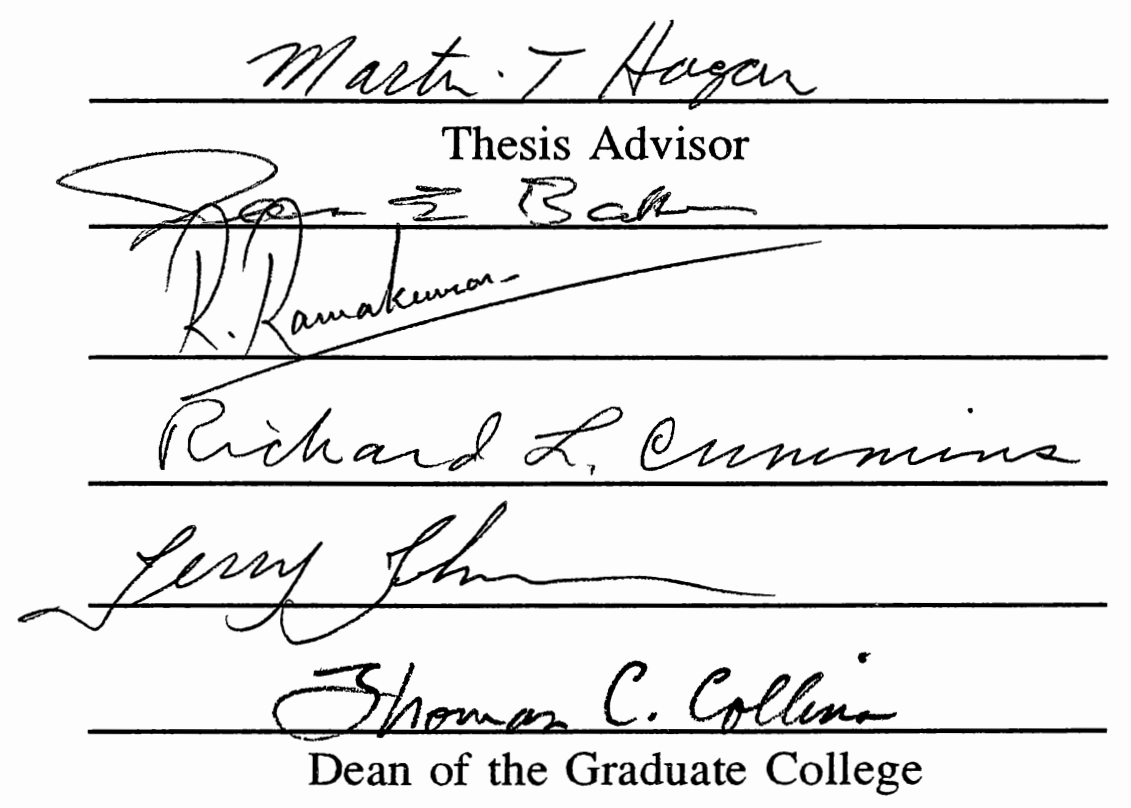




\section{ACKNOWLEDGEMENTS}

I wish to express my deepest gratitude to Dr. Martin T. Hagan, Chairman of my doctoral committee and my thesis advisor, for suggesting the problem, providing unstinted guidance, inspiration, and many hours of valuable time during my research.

I extend my sincere appreciation to Dr. Richard Cummins, Dr. Ramachandra G. Ramakumar, and Dr. Jerry Johnson, my doctoral committee members, for their interest and assistance. My thanks are due to Dr. Richard Cummins for making my graduate study at O.S.U possible and his continued assistance.

I am indebted to the Air Force Office of Scientific Research and the School of Electrical Engineering for their financial support of my graduate study and research. 


\section{TABLE OF CONTENTS}

$\begin{array}{lll}\text { Chapter } & \text { Page }\end{array}$

I. INTRODUCTION $\ldots \ldots \ldots \ldots \ldots \ldots \ldots \ldots \ldots \ldots \ldots$ 1-1

1.1 Problem Statement $\ldots \ldots \ldots \ldots \ldots \ldots \ldots \ldots \ldots 1-1$

1.2 Organization $\ldots \ldots \ldots \ldots \ldots \ldots \ldots \ldots \ldots \ldots \ldots$ 1-3

II. HUMAN PERFORMANCE MODELING ........... 2 - 1

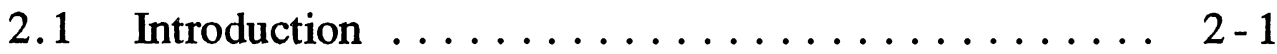

2.2 Manual Control Characteristics ........... 2-2

2.3 Operation of a Simple Manually-Controlled System ..................... 2-6

2.4 Analysis Techniques For Human Performance 2-10 2.4.1 Quasi-linear Controller Model ......... 2-11

2.4.2 Cross-Over Model ................. 2-14

2.4.3 Optimal Control Model ............ 2-20

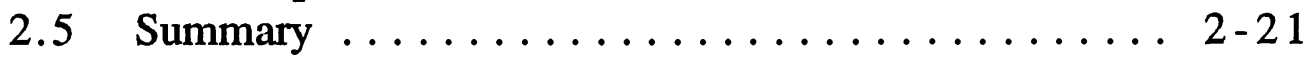

III. OPTIMAL CONTROL $\ldots \ldots \ldots \ldots \ldots \ldots \ldots \ldots \ldots . \ldots \ldots$

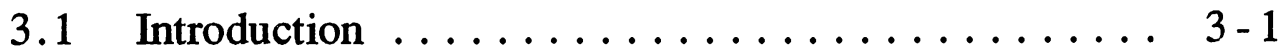

3.2 State Variable ................... 3-4

3.3 Linear Systems ................ 3-6

3.4 Linear Optimal Controller ............. 3-8

3.5 Noise Free Regulator Problems . . . . . . . . 3-10

3.5.1 Problem Statement ............. 3-10

3.5.2 The Solution ................ 3-12

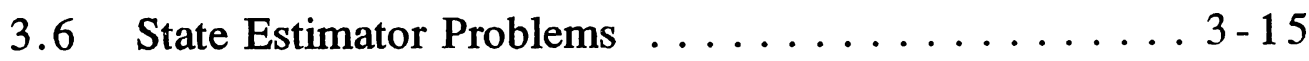

3.6.1 Optimal State Estimator .......... 3-17

3.6.2 The Solution of the Optimal State

Estimator .................. 3-19 
3.7 Optimal Controller for Stochastic Linear Systems ..................... 3-22

3.8 Optimal Tracking Problems .......... 3-24 3.8.1 Noise Free Tracking Problem ....... 3-24 3.8.2 Linear Optimal Stochastic Tracking Problem .................. 3-32

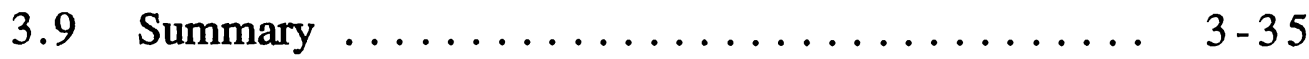

IV. OPTIMAL CONTROL MODEL (OCM) .......... $4-1$

4.1 Introduction ................. 4-1

4.2 Kleinman-Baron-Levison's Model ......... 4-3

4.2.1 General Descriptions \& Assumptions ... 4-3

4.2.2 Vehicle Dynamics, Task Description and Representation of Human

Operator's Limitations . . . . . . . . . . . 4-6

4.2.3 Basic Structure of the OCM ........ 4-10

4.2.4 The Solution Of OCM ........... 4-12

4.3 Modified OCM (Using Pade Approximantion for Delay Elements) ................ . 4-19

4.3.1 Pade Approximant ............. 4-20

4.3.2 State Space Model ............. 4-23

4.3.3 Solution of the Modified OCM ....... 4-26

4.4 Model Validation .................. 4-31 4.4.1 An Example of Aircraft Landing Approach ................. 4-33

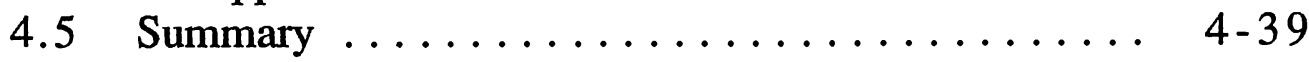

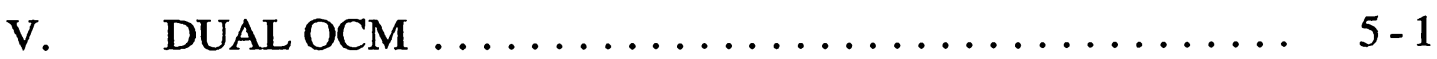

5.1 Introduction ................ 5-1

5.2 The Dual OCM and its Solution ......... 5-2

5.2.1 OCM1 Development .......... 5-3

5.22 OCM2 Development .......... 5-10

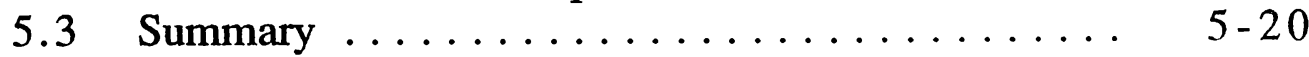


VI. Analytical Results $\ldots \ldots \ldots \ldots \ldots \ldots \ldots \ldots \ldots$ 6 6 .

6.1 Analytical Results of a Formation Flight Task 6-1

6.2 A Simple Optimal Tracking Problem ........ 6-20

6.3 A Simple Tracking Problem With

Intrasimulator Delay ............. 6-24

6.4 Simple Tracking With OCM ............ 6-32

6.4.1 Effect of $\tau_{2}$ on OCM Response ..... 6-36

6.4.2 Effect of $\tau_{2}$ on MSE .......... 6-37

6.5 Lower Order Dual OCMs' Results .......... 6-39

6.5.1 Analytical Results of a First Order

Dual OCM ................... 6-40

6.5.2 Second Order Dual OCM ......... 6-41

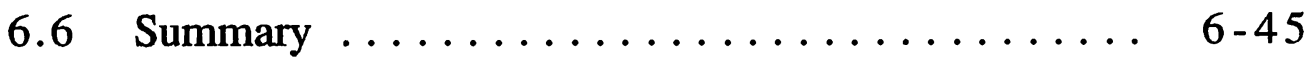

VII. An Introduction to an Air-to-Air Combat Task ..... 7-1

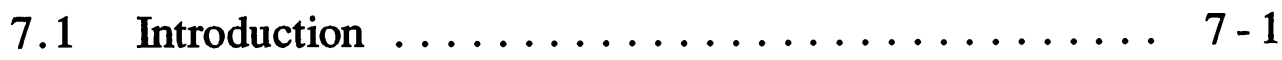

7.2 Differential Games ............... 7-3

7.2.1 Game theory .............. 7-3

7.2.2 Differential Games ............ 7-6

7.3 Linear Deterministic Pursuit-Evasion Game ... 7-8

7.3.1 The Solution ............... 7-10

7.3.2 An Example. Guidance Law

for Target-Interception . . . . . . . . . 7-14

7.4 Linear Stochastic Differential Games ........ 7-19

7.4.1 Introduction .............. 7-19

7.4.2 Problem Statement ........... 7-20

7.4.3 The Solution .............. 7-22

7.4.4 Delayed Commitment Solution ....... 7-27

7.5 An Example of a Stochastic Differential Game . . 7-31

7.6 Differential Games \& OCM ........... 7-38

7.6.1 Development of a Simple Air-to-Air

Combat Task ............... 7-38

7.6.1.1 Evader Development . . . . . . . 7-42

7.6.1.2 Pursuer Development . . . . . . . . 7-43

7.6.1.3 Development of Pursuer-Evader

System ................. 7-44 
Chapter

7.6.2 A Missile-Aircraft Example ....... 7-46 Summary $\ldots \ldots \ldots \ldots \ldots \ldots \ldots \ldots \ldots \ldots$ 7 -50

VIII. CONCLUDING REMARKS $\ldots \ldots \ldots \ldots \ldots \ldots \ldots \ldots, 8-1$

8.1 Conclusions .................. 8-1

8.2 Suggested Topics for Further Research ...... 8 8-3

8.2.1 Noisy-State Pursuit-Evasion Differential Games .............. 8-4

8.2.2 Non-Zero Sum Differential Games ..... 8- 8

8.2.3 Two-Target Qualitative Games ....... 8-6

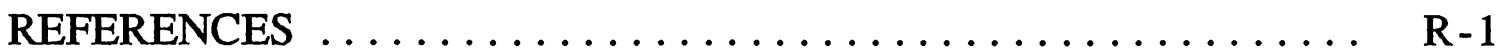

APPENDIX A - OCM SOLUTION $\ldots \ldots \ldots \ldots \ldots \ldots \ldots \ldots$ A-1

APPENDIX B - STABILIZABILITY OF THE SYSTEM . . . . . . . B - 1

APPENDIX C - SOLUTION OF A SIMPLE OPTIMAL

TRACKING PROBLEM $\ldots \ldots \ldots \ldots \ldots \ldots \ldots$ C-1 


\section{LIST OF TABLES}

Table

Page

2.1. Human operator's adaptation to plant dynamics $\ldots \ldots \ldots \ldots \ldots \ldots \ldots \ldots \ldots$ 2-19

4.1. Measured and Theoretical Mean Squared Values of Error, Error Rate and Control

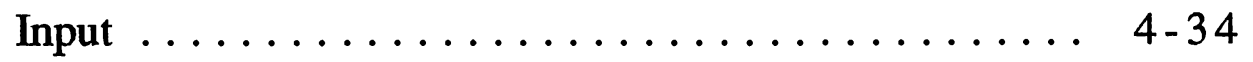




\section{LIST OF FIGURES}

$\begin{array}{ll}\text { Figure } & \text { Page }\end{array}$

2.1. General Scheme of a Manual Control System ....... 2-5

2.2. Major Internal Pathways of Human Operator ...... 2-5

2.3. A Simple Compensatory Manual Control System .... . 2-8

2.4. A Simple Pursuit Manual Control System . . . . . . . . 2-9

2.5. Typical Displays for Compensatory and Pursuit

Tasks ........................ 2-9

2.6. Quasi-linear Human Operator Model ........... . 2-12

2.7. Quasi-linear Model of Human in a Compensatory

Tracking Task ................. 2-14

2.8. Frequency Domain Configuration of Figure $2.7 \ldots \ldots$ 2- 16

2.9. The block-diagram of the Cross-Over model ...... 2-18

3.1. General Scheme of Estimation \& Control ......... 3-3

3.2. A Simple Black-Box Diagram of a Physical System .... . 3-5

3.3. Block-Diagram for a Continuous Linear Time Invariant Stochastic System ........... 3-7

3.4. General Representation Of A Control Problem ....... 3-12

3.5. Noise-Free Linear Regulator Controller . . . . . . . . . 3-13 
Figure

Page

3.6. Using estimator in control law $\ldots \ldots \ldots \ldots \ldots \ldots$ 3-17

3.7. Plant-Estimator Structure For Linear Stochastic Systems .................. 3-20

3.8. Optimal controller for linear stochastic systems ... . 3-23

3.9. Block-Diagram Of Linear Optimal Stochastic Regulator................... 3-27

3.10. Block-Diagram of the Regulator Control Of The Augmented System $\underline{x}_{T}$ (Tracking Problem) ... . 3-30

3.11. Block-Diagram Of Optimal Tracking Problem ...... 3-31

3.12. A Linear Optimal Stochastic Tracking Problem ..... 3-35

4.1. OCM Model Structure $\ldots \ldots \ldots \ldots \ldots \ldots \ldots \ldots$ 4-5

4.2. Perceptual Submodel for Human Operator . . . . . . . . 4-11

4.3. The Controller Submodel ............... 4-11

4.4. Frequency Domain Representation of the OCM . . . . 4 4-17

4.5. Controller System With Perceived Output Variables .................... 4-20

4.6. Input/Output Relationship for Delay $\tau \ldots \ldots \ldots \ldots$ 4-24

4.7. Equivalent Configuration of Figure $4.5 \ldots \ldots \ldots \ldots$. . . 27

4.8. Modified OCM Structure $\ldots \ldots \ldots \ldots \ldots \ldots \ldots \ldots$ 4-30

4.9. Block Diagram Of Example1 Of Ref. [55] ....... 4-34 
Figure

4.10. Experimental Validation Of KBL's Model in a simple Tracking Task, $\mathrm{Y}_{\mathrm{c}}=1 / \mathrm{s}$., Taken From Reference [55] . . . . . . . . . . . . 4-35

4.11. Controlling Aircraft Against Wind Gust During Landing ................ 4-36

4.12. Frequency Plots for Open-Loop System Figure $4.11 \ldots \ldots \ldots \ldots \ldots \ldots \ldots \ldots . \ldots \ldots$ 4. 37

4.13. The Human Operator as a Phase Lead Compensator .................... 4-38

4.14. Resonant Peak In Human Operator Transfer Function ..................... 4-39

5.1. Dual OCM Structure for Formation Flight ....... 5-3

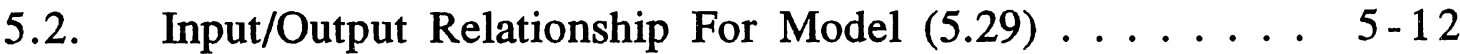

5.3. Tracking Configuration of the Dual OCM ......... 5-14

5.4. Using Modified OCM in Figure $5.3 \ldots \ldots \ldots \ldots \ldots$ 5-14

5.5. Block-Diagram Of Modified OCM For Wingman .... 5 5-17

5.6. Reconfiguration Of Figure $5.1 \ldots \ldots \ldots \ldots \ldots \ldots$ 5-21

6.1. Dual OCM For a simplified Formation Flight Problem ............... 6-3

6.2. Vehicle Dynamics in the Dual OCM .......... $6-4$

6.3. Effect of Intra-simulator Delay on System Performance................. 6-6

6.4. Comparison Of The Errors Plotted In Figure $6.3 \ldots 6$ 6- 7 
Figure

Page

6.5. The Effect of $\tau_{1}$ on Mean Square Error ....... 6-9

6.6. The Effect of $\tau_{2}$ on Mean Square Error ........ 6-14

6.7. Mixed Effects Of $\tau_{1}$ And $\tau_{2}$ On Mean Square Error ... 6-18

6.8. Simple optimal tracking problem ........... 6-21

6.9. The effect of plant parameter $\tau_{2}$ on optimal mean square tracking error ...........6 6-22

6.10. Simple Optimal Tracking Problem With an Intra-Simulator Time Delay $\tau_{3} \ldots \ldots \ldots \ldots$ 6-25

6.11. Actual Error and Delayed Error Versus. $\tau_{3} \ldots \ldots \ldots 6-28$

6.12. Magnitude and Phase Of $\mathrm{H}_{1}^{\mathrm{d}}(\mathrm{s}) \ldots \ldots \ldots \ldots \ldots \ldots$ 6-29

6.13. Phase and Magnitude of $\mathrm{H}_{2}(\mathrm{~s}) \ldots \ldots \ldots \ldots \ldots \ldots$ 6-30

6.14. A simple Tracking Problem With OCM ......... 6-33

6.15. The Effect of Plant Parameter "a" on MSE ....... 6-34

6.16. Frequency Plots Of $\mathrm{H}_{2}(\mathrm{~s}) \& \mathrm{H}_{1}$ (s)vs.

$$
\tau_{1}\left(\mathrm{a}=\tau_{2}=0.15, \tau_{3}=0\right) \ldots \ldots \ldots \ldots \ldots \ldots \ldots 6 \text { 6 } 6 \text {. } 35
$$

6.17. Frequency Plots of OCM $\left(a=\tau_{1}=0.15, \tau_{3}=0\right) \ldots \ldots 6-36$

6.18. The Effect of Internal Delay $\tau_{2}$. on MSE

$$
\left(\mathrm{a}=\tau_{1}=0.15\right) \ldots \ldots \ldots \ldots \ldots \ldots \ldots \ldots \ldots \ldots \ldots \ldots \ldots
$$

6.19. Reconfiguration of Figure 6.14 (when delayed error is minimized) $\ldots \ldots \ldots \ldots \ldots \ldots \ldots \ldots$ 6-39 
6.20. Effects of Delays on MSE in a First Order Dual OCM .................... 6- 6-42

6.21. Effect of Delay $\tau_{2}$ on MSE for a Second Order Dual OCM .................... 6-43

6.22. Effect of Delay $\tau_{1}$ on MSE for a Second Order Dual OCM .................... 6-44

7.1. Simulation of a Simple Pursuit-Evasion (Target Interception) Problem . . . . . . . 7-18

7.2. Stochastic Pursuit-Evasion Structure ........ 7-24

7.3. The Feedback Strategy Gains ........... 7-34

7.4. Terminal Miss Versus Time, $\mathrm{V}_{\mathrm{x}}=1 \ldots \ldots \ldots \ldots$ 7-35

7.5. The Error Variance of The Evader's Estimate ..... 7-36

7.6. The Relative Payoff as a Function of $v_{x} \ldots \ldots$ 7-37

7.7. The Relative Payoff $\ldots \ldots \ldots \ldots \ldots \ldots \ldots$ 7-37

7.8. Block Diagram of a Simple Air-to-Air Combat Task ............... 7-39

7.9. The Effect of $\tau_{3}$ on a Missile-Aircraft Problem ... 7-49

7.10. The Effect of $\tau_{2}$ on Missile-Aircraft Problem, $\tau_{3}=0 . \ldots$ 7-49 


\section{NOMENCLATURE}

\begin{tabular}{|c|c|}
\hline Matrices & - Upper case alphabet \\
\hline Vectors & - Underscored lower case alphbet \\
\hline Scalars & - Lower case alphabet \\
\hline$I_{n}$ & - $(\mathrm{n} \times \mathrm{n})-$ identity matrix \\
\hline$A^{\prime}$ & - Transpose of a matrix \\
\hline$A^{-1}$ & - Inverse of a matrix \\
\hline Diag [.] & - Diagonal matrix \\
\hline Det $[\mathrm{A}]$ & - Determinant of a matrix \\
\hline $\operatorname{Tr}[\mathrm{A}]$ & - Trace of a matrix \\
\hline$\|\|$. & - Matrix or vector norm \\
\hline |. & - Absolute value \\
\hline$\{\cdot\}$ & - Set \\
\hline$\epsilon$ & - Element of a set \\
\hline$\neq$ & - Equality does not hold \\
\hline$\equiv$ & - Identity \\
\hline$\leq$ & - Less than or equal to \\
\hline$>>$ & - Far greater than \\
\hline$\cong$ & - Approximately equal to \\
\hline & \\
\hline
\end{tabular}




\section{CHAPTER I}

\section{INTRODUCTION}

\subsection{Problem Statement}

There is a great deal of interest within the Air Force community in developing a system for networking multiple high-performance aircraft simulators. Interest is especially high because of the successful testing of SIMNET, a network developed by BBN under sponsorship of the Defense Advanced Research Projects Agency (DARPA) and the United States Army. SIMNET is a research project whose goal is to build a large-scale network of interactive combat simulators.

One of the results of this research has been the development of a SIMNET protocol. This protocol defines an environment in which a variety of simulator types can be interfaced, with minimum additional hardware and software requirements for the individual simulators. The protocol defines the types and formats of messages which can be passed over the network. It also defines the manner in which such things as vehicle appearance, location, and orientation, weapons fire, etc., are communicated.

One of the key questions to be answered during the development of a simulator network is "How much delay can be tolerated in inter-simulator communication?". The SIMNET experience can provide only limited answers to this question, because of the 
requirements for high-performance aircraft simulation. The original SIMNET tests involved tank simulators. Tank simulators have relatively slow response times and modest accuracy requirements, therefore delays caused by the operation of the network would not be as critical.

Although the adverse effects of delays in single flight simulators have been discussed fully in the literature [1-7], less effort has been made in the analysis of delay for networked high-performance flight simulators [8]. For the purposes of this research the key characteristic of SIMNET, or any other networking protocol, is the network delay. There are two components to the network delay. The first component is transport delay, caused by the physical distances over which the signal must travel. For local area networks this delay is small, but for long-haul satellite networks the delay can be several hundred milliseconds. The second component is the delay caused by limitations in network bandwidth. As the number of simulators in the network grows, it will become more likely that messages will be stuck in queues for significant periods. These delays are especially problematic, because they will vary with time.

The SIMNET protocol has attempted to deal with the second type of delay by limiting the amount of traffic on the network. Vehicle information is transmitted only when the vehicle state has changed significantly. In order to mitigate the effects of both types of delay, a time stamp is added to each message so that the receiver will know when the message was sent. (Of course, this requires all simulators to be time-synchronized.) 
In addition, the SIMNET protocol requires each simulator to have a simplified (dead-reckoning) model of every vehicle in the simulation, in order to extrapolate vehicle states between updates. Each simulator also has a dead-reckoning model of itself. Updates are transmitted whenever the dead-reckoning model state differs by more than a specified amount from the true vehicle state.

These measures appear to be satisfactory solutions to the delay problem, when networking simulators for relatively slow-moving vehicles that require limited fidelity. However, there is concern that these measures may not be sufficient when networking high fidelity simulators for high-performance aircraft.

There appear to be four tasks in which delays might cause problems in networks of high-performance aircraft simulators: air-toair combat, formation flight, air-to-air refueling, and target handoff. For the purposes of this research the formation-flight task was the major task chosen in the first phase of study. It is the most amenable to standard analysis techniques, and therefore was most appropriate for the initial study. Some work has also been done on the more challenging air-to-air combat task.

\subsection{Organization}

In order to analyze the networked-simulator problem, it is necessary to have a mathematical model of the pilot. There have been two basic approaches for modeling humans performing manual control tasks: the cross-over model and the optimal control model (OCM). Chapter II will discuss the field of human performance modeling, and 
will provide brief descriptions of these two techniques. The OCM will be used to analyze the networked-simulator problem, and it will be described in detail in Chapter IV. The necessary optimal-control background for the OCM will be presented in Chapter III.

The OCM has been used in the past to analyze the effects of delays within single simulators, but has not been used to analyze the effects of delays between networked simulators. The formation flight task can be analyzed with a modification to the standard OCM. This modification is called the "dual" OCM, and is described in Chapter V.

The basic formation-flight task has been carefully analyzed, and a number of interesting phenomena have been found. These phenomena are described and explained in Chapter VI. As a logical extension, the second phase of this dissertation studies the air-to-air combat task, which is more sensitive to the effects of the delays between simulators. Chapter VII considers the feasibility of developing an OCM air-to-air combat model. This chapter first presents a review of the literature on pursuit-evasion differential game theory, then develops an OCM model for a simple air-to-air combat task, and finally analyzes a missile-aircraft problem and summarizes its main results. Chapter VIII summarizes the principal results of this dissertation and contains suggestions for further research. 


\section{CHAPTER II}

\section{HUMAN PERFORMANCE MODELING}

\subsection{Introduction}

Among primates, the human being is recognized as a tool user. For the whole of his/her history, the human, in order to survive, has used simple hand tools for eating, building and fighting.

During the last four decades, humans have built very complex technological systems such as sophisticated vehicles and production machines, and he is expected to control these systems. As a result, man and machine have to come together; this is called the manmachine interaction.

In order to design the interface between man and machinery, one needs to define precisely the individual tasks which must be executed by the human, and by the machine. These task assignments have to be the best, i.e. optimal in some sense. The optimization will occur if the capabilities and limitations of both the human and the machine are considered carefully $[11,12]$. In other words, in order to have an optimum man-machine performance, it is necessary to consider the demands that a certain individual task imposes on the human's limited resources [13].

In any system design, in order to describe or to optimize a system, one first needs to model the system mathematically. These 
models must describe the dynamic behavior of both the human and the controlled system. Furthermore, they must be able to predict effectively the human performance on the system which is controlled by the human operator, because the total human-system behavior is the main concern of system designers. In order to know analytically the total man-in-the-loop performance, the development of mathematical models for the human operator are quite necessary, since the machine's performance quality itself cannot provide enough information about the quality of overall system performance.

To have a correct and sufficient control of a machine, it is assumed that the human operator is familiar with the dynamics of the machine, the statistics of the disturbances subjected to the machine and the overall task requirements. Practically this assumption means that in the development of human performance modeling, one must provide the human operator an internal model of the system and its environment [14-22]. For more about human performance modeling and its role as an information processor or adaptive controller in man-machine systems, one may refer to [2336].

\subsection{Manual Control Characteristics}

Human adaptabilities have been studied fully in the literature [15,24,37-38], and it has been found that human is capable of adjusting his characteristics to meet system requirements. These human abilities provide manually-controlled system designs with a 
large degree of flexibility which is somehow unreachable in fullyautomatic control system designs [39-41].

Manual control is defined as follows:

A man who perceives, via his senses, information about the desired states and the actual output states of a given system, separately or in combination, whereby he is expected to manipulate some mechanical equipment, i.e. control stick, so as to minimize some appropriate performance criteria [40].

Manual control theory has a powerful application for monitoring complex vehicles, such as aircraft, automobiles, bicycles, etc., which have limited bandwidth and operate in a well-defined optimal fashion, and in which the human operator needs to control only a few input variables [15,40-41].

Basically, a manual control system, illustrated in Figure 2.1, consists of three key elements:

1- Plant dynamics, $\mathrm{H}_{\mathrm{c}}$

The equations describing the operation (motion) of the controlled system. (This is the machine which is being controlled by the human operator.)

2- Task requirements (objectives)

It may be defined by a scalar dynamic equation evaluating the relative quality of the closed-loop performance.

3- Human operator dynamics

It represents a practical, usable, mathematical model of the human operator. 
Inputs to the overall system are:

1- Random unwanted noises acting on the controlled system (e.g., wind gust and observation noises).

2- Task input commands

They are related to task objectives, e.g., target maneuvering for an air-to-air combat task.

3- Remnants

They are random noises produced by the human operator, e.g., responses not related with any other system inputs.

Figure 2.1 shows that the human operator behaves as a controller. Later it will be seen that the application of optimal control theory in modeling the human operator results in a time-varying optimal controller; it means that the human operator acts like a time dependent optimal controller.

Figure 2.2 represents some of the versatile features of the human operator controller as an information processor. These figures are based on Figure 1 in [41].

The neuromotor subsystem, which is actually the human operator's output mechanism, consists of muscle, limb, spindle and manipulator dynamic elements; their properties depend on the controlled system and the task objectives [41]. 
Disturbances

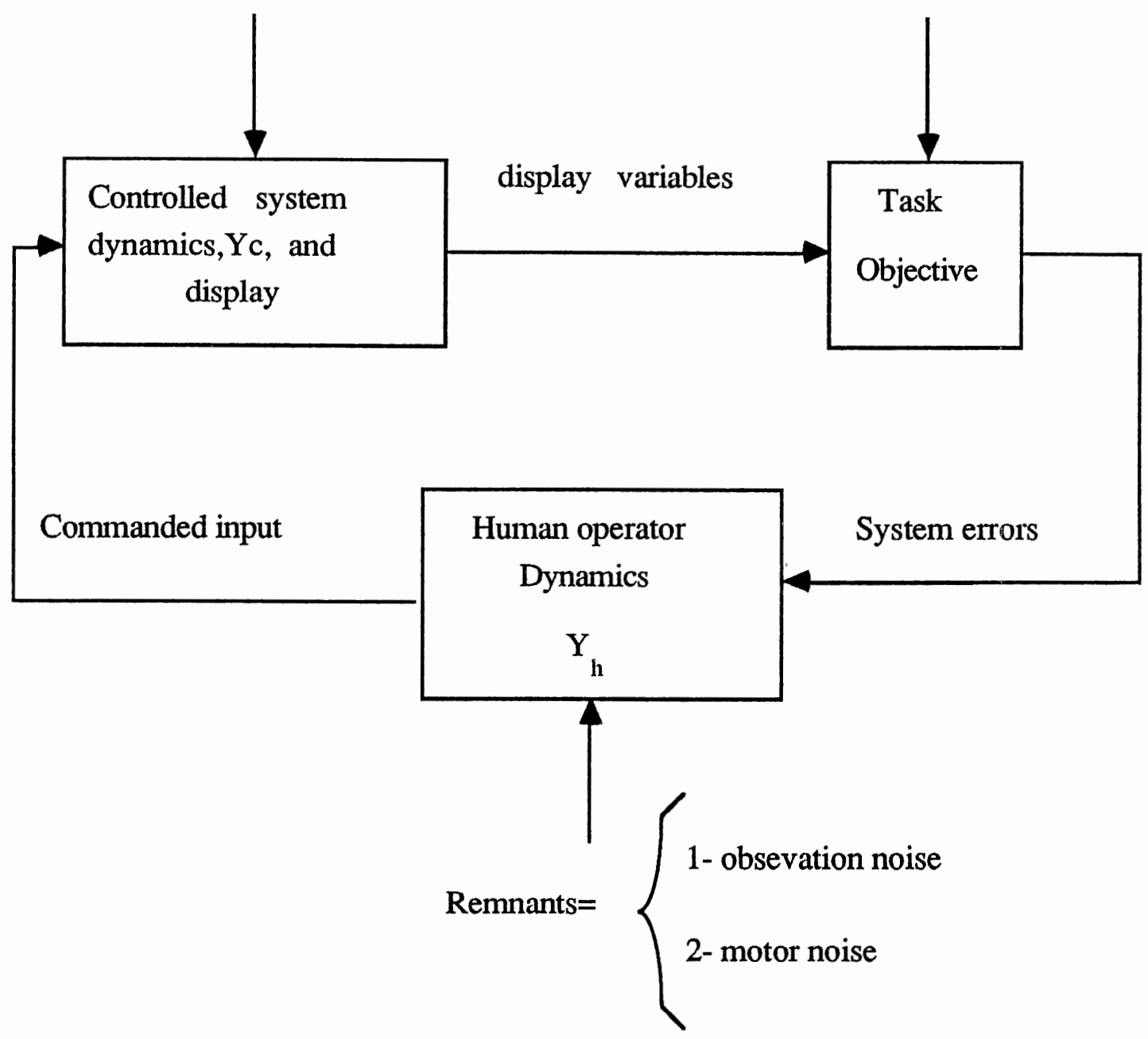

Figure 2.1. General scheme of a manual control system

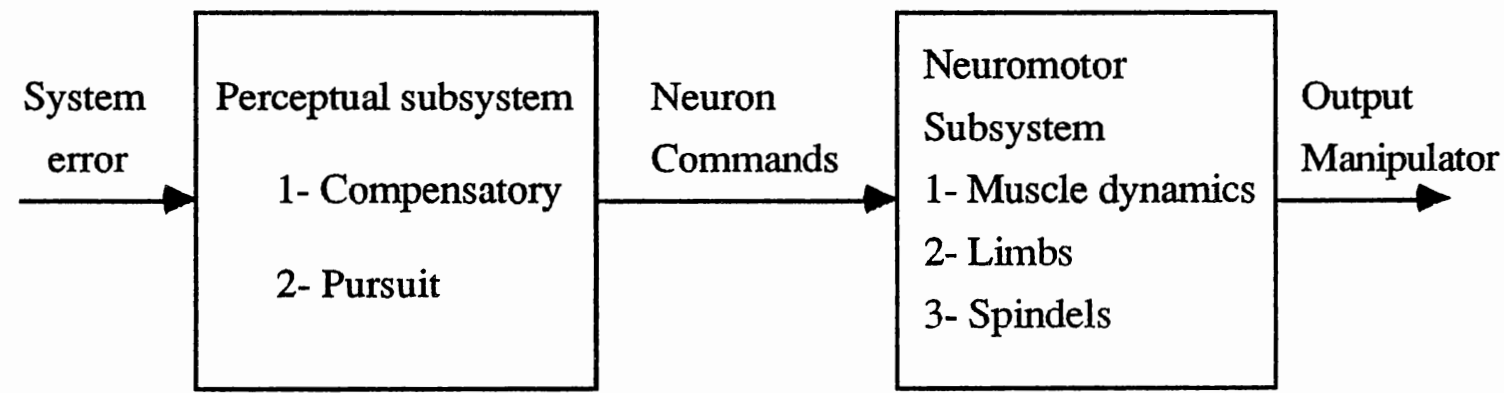

Figure 2.2. Major internal pathways of the human operator 
The perceptual subsystem consists of two major pathways determining the human control operation structure: compensatory and pursuit pathways. In compensatory pathways, the human operator observes only the error between his input and output, whereas with pursuit, the human observes both his input and output separately; in both cases the human operator's task is to minimize the error. These two pathways are discussed in the next section in detail.

\subsection{Operation of a Simple Manually- Controlled System}

The human operator is considered as an adaptive servomechanism element attempting to detect the feedback error between system input and output and to perturb the system via some manipulator devices in order to reduce the error. In other words, the human operator is solving a tracking problem, which is defined clearly as a movement process in which the human operator keeps trying to minimize some degree of mismatch between a desired output, an external input quantity, and the actual output, the output of the controlled system. For example, a pilot tries to keep an aircraft on a specified flight path.

The tracking ability of the human operator makes him an especially unique element in manually-controlled system designs. It has been shown that the human operator is able to use the statistical characteristics of the input signal and the dynamic characteristics of the other elements of the system to adjust (shape) his response 
characteristics in order to match the behavior of the control situations [16-17,41-44].

Furthermore, when tracking a low frequency sinusoid, the human operator is able to predict the future of the sinusoid and compensate for his inherent response lags. In other words, the human operator is able to make his response approximately in phase with sinusoidal inputs [45]. For stochastic signals, it has been shown that human response lags the input $[41,45]$.

Human operator characteristics are not fixed, and it has been found that the stochastic variations in his characteristics are due to the external stimuli, not due to the input signals; they are called remnant and treated as if they were noise [15], see Figure 2.1.

The most important characteristics of the human operator behavior may be enumerated as [14]:

i. Reaction-time delay

ii. Low frequency bandwidth

iii. Relatively good amplitude linearity.

Major sources of the reaction time delay are retina excitation, (roughly about $0.02-0.04$ second), nerve condition (between 0.01 and 0.02 second), muscle contraction (in the range of $[0.02,0.04]$ seconds), and finally the time required for processing, which is basically dependent on the task requirements [14-15,40]. In a simple tracking task, the reaction time delay is about 0.1 to 0.4 second $[14,15]$. Both the visual and muscular systems possess a maximum of 10 cycles per second in their frequency responses and in visual- 
manual control tasks, the controlled elements cannot move faster than three cycles per second [14,39]. Further, it has been shown that for accurate tracking, one needs to consider input signals whose power is mostly concentrated below one cycle per second in their spectrum [15].

A simple manual control system includes a controlled system, a human operator as a controller and a display. The two most important types of manual control tasks, pursuit and compensatory, are shown in Figures 2.3 and 2.4 respectively. In the compensatory task, only the error between the input and the output is displayed, as shown in Figure 2.5. The human operator's task in this case is to reduce the mismatch error by keeping the circle around the dot (see Figure 2.5).

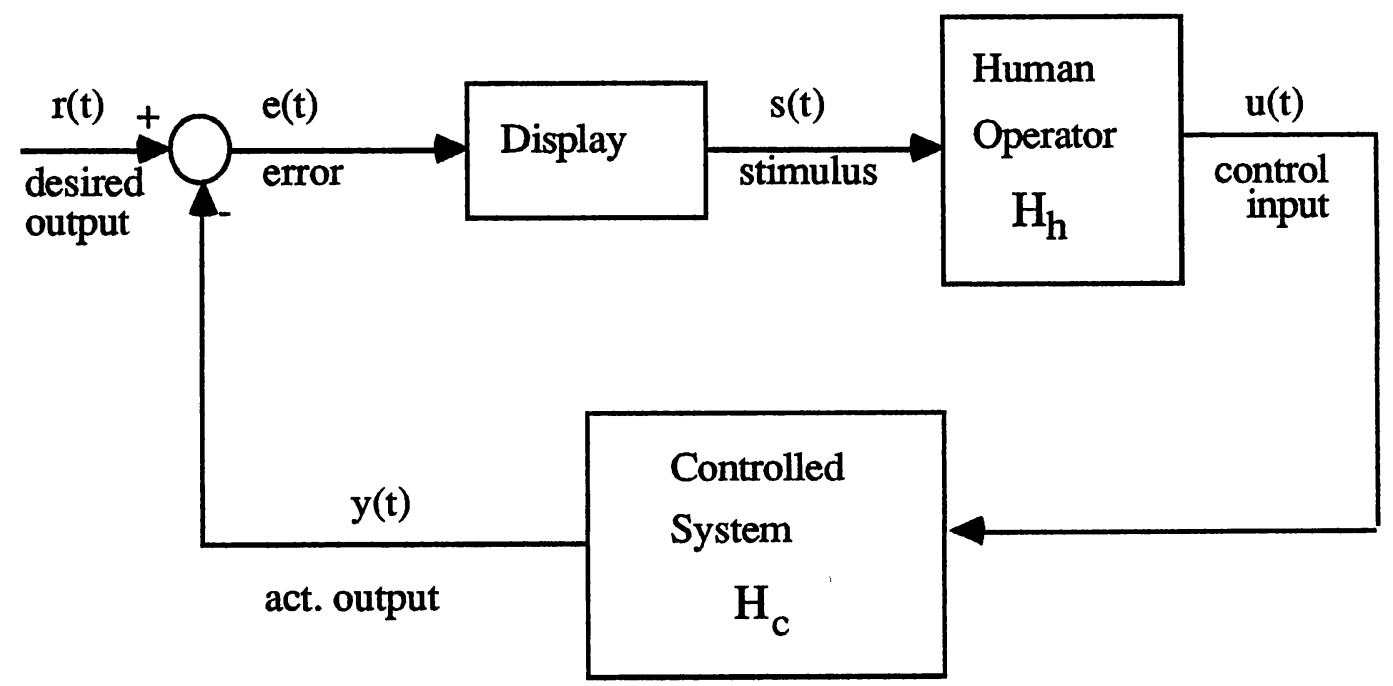

Figure 2.3. A simple compensatory manual control system 


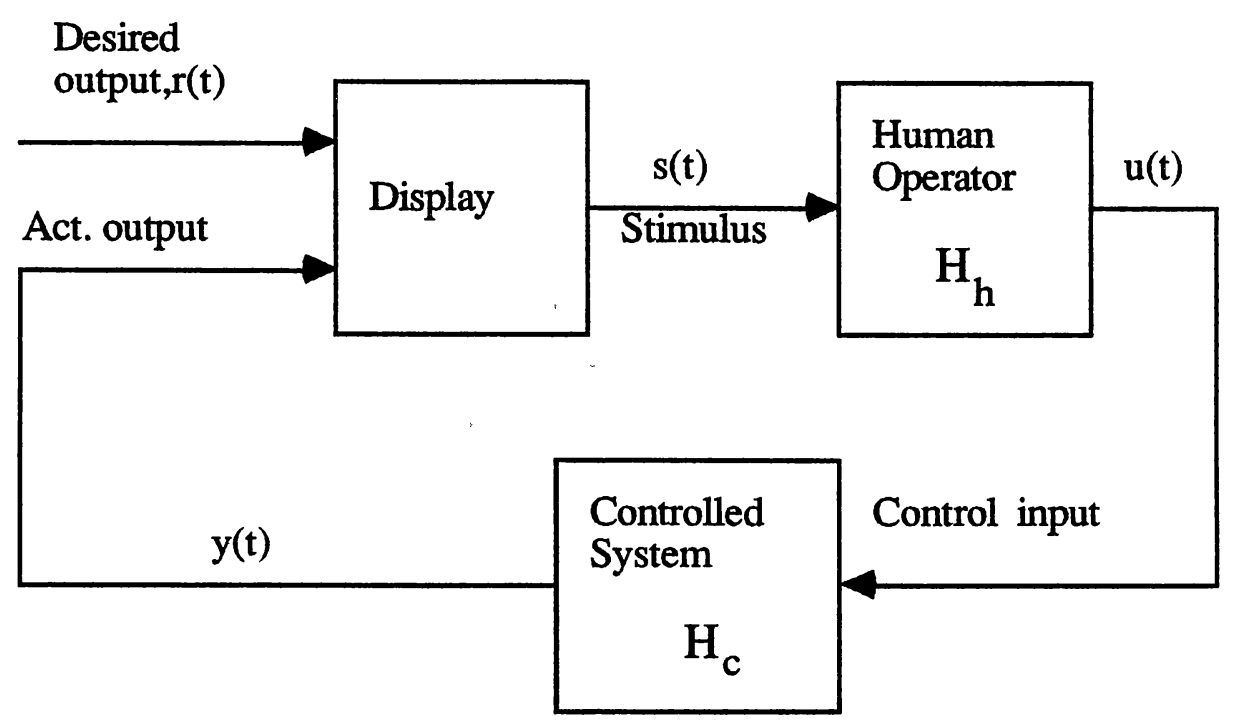

Figure 2.4. A simple pursuit manual control system

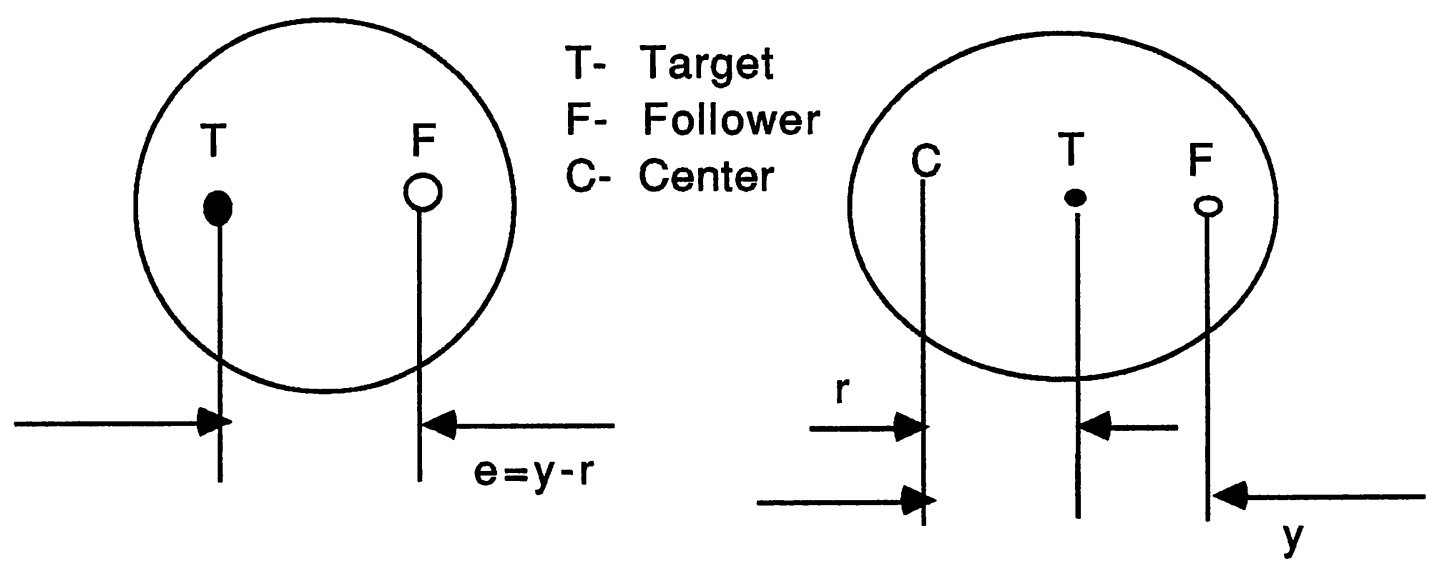

For Compensatory

For Pursuit

Figure 2.5. Typical displays for compensatory and pursuit tasks 
In the pursuit system, both the input and the output are displayed separately, and the human's task is to follow the target by keeping the circle around the dot. It is clear that the pursuit system will perform better than the compensatory system, because of the direct availability of the input. This helps the human operator to predict the future of the input and to compensate for his inherent lags (e.g., reaction-time delays) [39-40].

\subsection{Analysis Techniques for Human Performance}

No physical system is really linear. The human operator, as a complex physio-psychological system, functions in a non-linear fashion [15,40-41]. The preceding section briefly reviewed the human's nonlinearities that strongly depend on the task variables, including system parameters, statistics of external disturbances, etc.

Consequently, the well-known classical techniques for describing control systems will not work for manually-controlled systems because of the human operator's nonlinear characteristics. Further, we have to deal with the structure of the family of the human operator characteristics. This structure is obtainable via measurement of the human operator performance in several different tracking situations [15,46-47].

Between 1945 and 1970, more than five hundred papers and technical reports were published related to human operator modeling, applying nonlinear analysis techniques to describe the 
characteristics of manually-controlled systems, i.e human tracking performance. These models can be rooted in two broad categories:

i) Classical control theory

ii) Modern control theory

Classical control theory applies closed-loop stability analysis to form describing functions of the human operator, as a controller, in order to predict the operator's output. Whereas the modern control theory uses state space analysis techniques to model the human operator $[17,48]$.

Among these models, the quasi-linear describing function model, Cross-Over Model [15], and the Optimal Control Model (OCM) [49], have been been recognized as the two most powerful tools in the representation of the human operator as a servo-element in man-machine systems. These two models will be discussed in section 2.4.2 and Chapter IV in detail.

Since both types of models, Cross-Over and OCM, represent the human operator in the man-machine system as a quasi-linear controller, the following section describes quasi-linear model properties briefly.

\subsubsection{Quasi-Linear Controller Model}

Quasi-linearization is a process which represents the characteristics of a non-linear system by a constant differential equation, linear transfer function $\mathrm{H}(\mathrm{s})$, and a stationary noise generator, $n(t)$. The constant parameters of $H(s)$ depend on the task 
variables including input signal, controlled element and other variables related to the non-linear system $[40,48]$. In other words, $H(s)$ possesses different parameters for different inputs, $r(t)$, and controlled elements, $H_{c}$. Further, they are assumed to be constant during any tracking run.

It has been found that quasilinearization is able to model the human operator's non-linearities, because the human's main deviation from linearity happens if the input statistics or the system parameters change $[15,39,40]$.

Figure 2.6 shows a block-diagram of a quasi-linear humanoperator model. The remnant, $n(t)$, represents the following nonlinearities or variabilities in the human's behavior:

i) Observation noise due to inaccurate measurements

ii) Motor noise due to inaccurate motor control

iii) Non-stationary behavior of the operator ( the parameters of $H(s)$ vary with time).

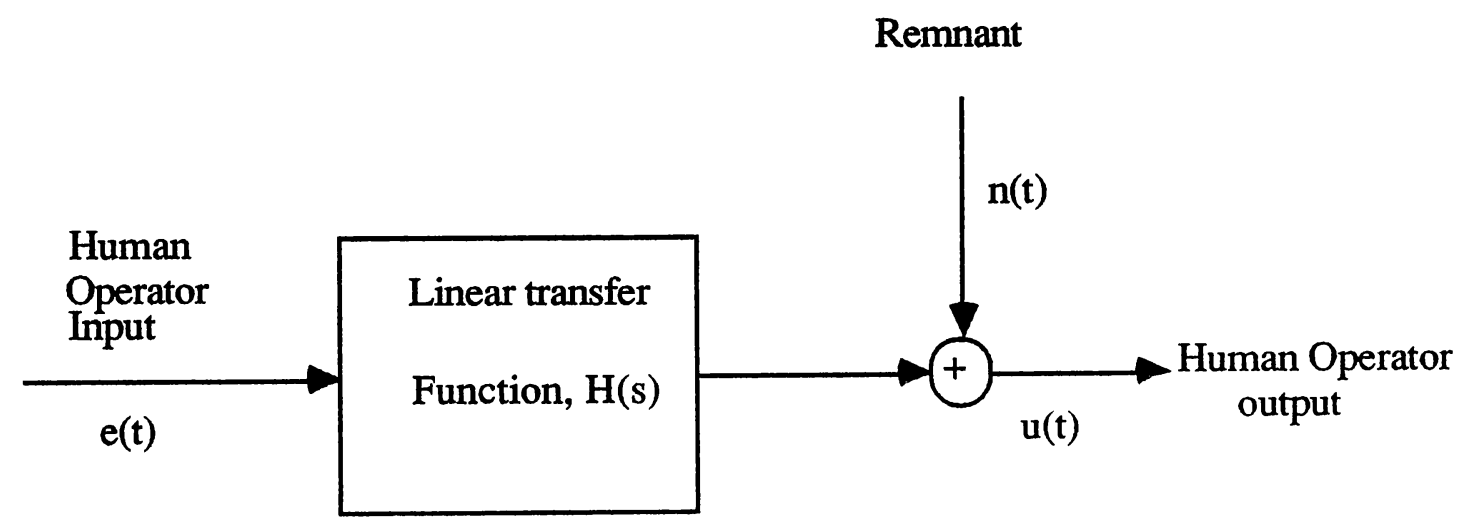

Figure 2.6. Quasi-linear human operator model 
In other words, the external noise signal, $n(t)$, represents the elements of the human behavior which cannot be correlated with the input, and which cannot be generated by a linear operation on the input $[49,50]$. Further, it has been shown [47] that the remnant occupies the longest portion of the human response for the tracking task, and it is independent with the input signal, e(t). Moreover, it possesses a smooth spectrum without sharp peaks $[39,46]$.

In order to measure the performance of the overall system, we need to compute (estimate) the spectral densities $H(f)$ and $N(f)$, see Figure 2.7, which can be determined from the measurements of the input and the output through the application of statistical techniques $[39,40]$.

The describing function analysis technique is a kind of transfer function technique which describes mathematically the frequency characteristics of non-linear systems which closely act like linear systems, for certain classes of inputs such as sinusoidal inputs [40]. The most famous describing function model is the Cross-Over model which is discussed in the next section. 


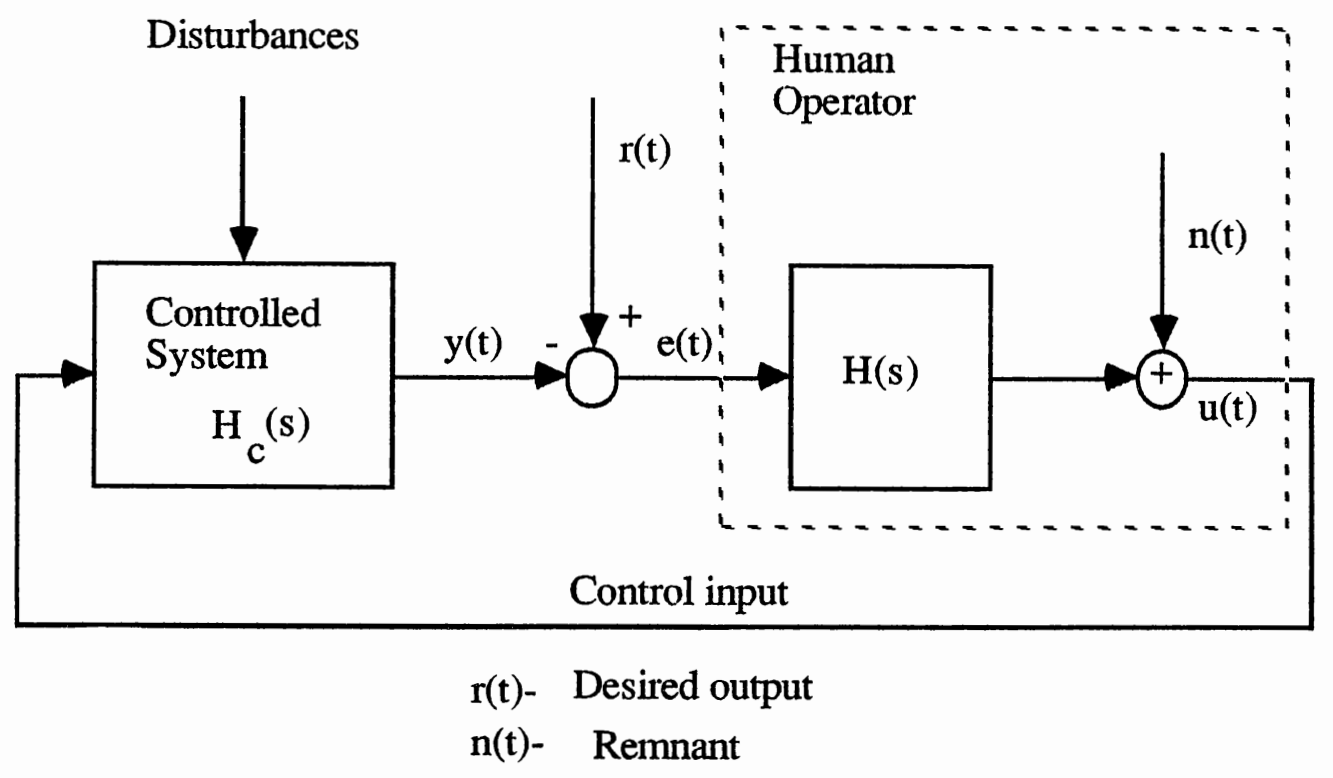

Figure 2.7. Quasi-linear model of human in a compensatory tracking task

\subsubsection{Cross-Over Model}

As mentioned earlier, because of certain nonlinearities in human operator, the structure of the family of human behavior can only be approximately formulated. This can be done only by measuring human performance in several different tracking tasks and by applying quasilinearization. McRuer and his coworkers in 1957 represented the family of human behavior with the structure given in Equation 2.1. They developed this model by performing of many studies on the findings of the previous researchers who had 
measured the human behavior in single-axis compensatory tasks $[15,40,51]$.

$$
H(s)=\underbrace{\mathrm{k} \frac{1+\mathrm{T}_{1} \mathrm{~s}}{1+\mathrm{T}_{2} \mathrm{~s}}}_{\text {Equilization term }} \times \underbrace{\frac{\mathrm{e}^{-\tau \mathrm{s}}}{1+\tau_{\mathrm{n}} \mathrm{s}}}_{\text {Limitation term }}
$$

$\mathrm{H}(\mathrm{s})$ is the linear transfer function in the quasilinear manual control system, illustrated in Figure 2.8, with

$$
\mathrm{U}(\mathrm{s})=\mathrm{H}(\mathrm{s}) \mathrm{E}(\mathrm{s})+\mathrm{N}(\mathrm{s}) \text {. }
$$

Herein, the describing functions are written in terms of the frequency operator $s=j w$ instead of the general complex form $s=\alpha+j \omega$.

The first term (equalization term) in Equation (2.1) represents the human adaptabilities by which he tries to optimize his control strategy to match a given situation. This term is strongly dependent on the controlled system and task situations. The limitation term in (2.1) represents the human operator's limitations, including reaction time delays and neuro-motor dynamics.

Equation (2.1) is called a general analytical/verbal describing function model for the human operator [12,14,50,53]. It is analytical because of its general dynamical form and it is verbal because it allows us to adjust its parameters so that the model can be an accurate estimate of the human operator for a specified situation.

In equation (2.1), $\mathrm{K}$ is an open loop gain which can be adjusted by the operator, and $T_{1}$ and $T_{2}$ are time constants corresponding to 
lead and lag coefficients, respectively. The human operator will adjust these variables so that the overall closed loop system satisfies some prespecified performance criterion, e.g. the highest open loop gain $\mathrm{k}$ with a phase lead less than 180 degrees, and so that the closed loop system possesses a phase margin between 60 and 100 degrees.

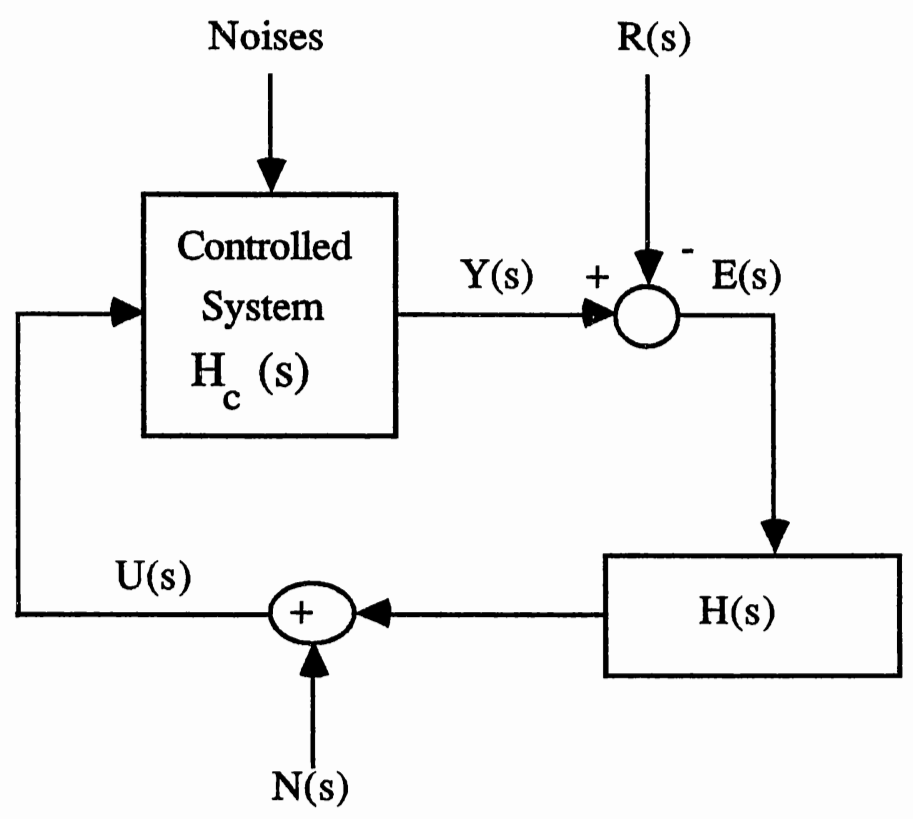

Figure 2.8. Frequency domain configuration of Figure 2.7

The time constant $\tau$ represents a reaction-time delay and $\tau_{n}$ represents a time constant coefficient of a first order lag in the neuromuscular system. Furthermore, it has been shown that $[12,50,52]$,

i) $1 \leq \mathrm{k} \leq 100$, 
ii) $\tau_{\mathrm{n}} \cong 0.2$ second,

iii) $0.12<\tau<0.2$ seconds.

Therefore, Equation (2.1) may be recognized as an implicit internal model of human operator. This model is obtained based on the stability criterion of the entire closed-loop man-machine system $[15,28]$.

Servo-control theory says that for good control dynamics, the open loop transfer function, $\mathrm{G}(\mathrm{s})=\mathrm{H}(\mathrm{s}) \mathrm{H}_{\mathrm{c}}(\mathrm{s})$, should behave as an integrator near the crossover frequency (the frequency where the gain of $G(s)=1)$; it means that as the frequency of the open loop moves toward the crossover frequency, the phase lag should remain less than 180 degrees in order to guarantee the stability [12,22,39$40,50]$.

For manual control systems, [15,50] shows that the following model possesses the property of a good servo.

$$
G(s)=H_{c}(s) H(s)=\frac{k w_{c}}{s} e^{-\tau s}
$$

Where $G(s)$ represents the open-loop transfer function and $W_{c}$ denotes the cross-over frequency. The model formulated by equation (2.2) is called the cross-over model. This equation says that

$|\mathrm{G}(\mathrm{s})|>>1$ for $\mathrm{w}<<\mathrm{w}_{\mathrm{c}}$

and

$|G(s)|<<1$ for $w>>w_{c}$ 
Hence, for forcing inputs with bandwidth, $\mathrm{W}_{\mathrm{e}}$, less than crossover frequency, $\mathrm{W}_{\mathrm{c}}$, the cross-over model behaves as a good servo, since for $w<W_{c}$ the open-loop phase lag is less than 180 degrees (no risk of instability) the open loop gain becomes very big, and most of the disturbances will be filtered, because for high frequencies $w>W_{c}$ the open loop gain becomes very small. Figure 2.9 shows the CrossOver model block-diagram for the compensatory manual control system of Figure 2.3. Ref. [41], by spectral analysis of experimental data, shows that the parameters of Cross-Over model, $k \& t$, are strongly dependent on input bandwidth and subject practice.

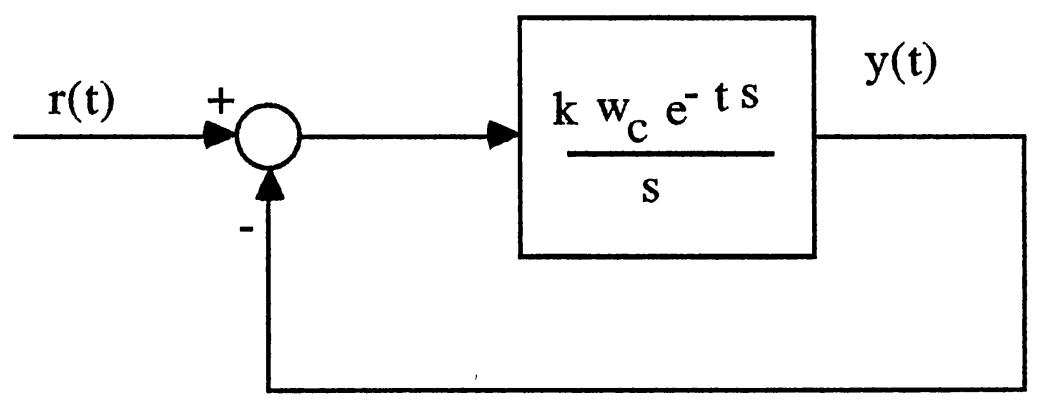

Figure 2.9. The block-diagram of Cross-over model

Starting with equation (2.1), the human operator attempts to adjust the values of adjustable variables $k, T_{1}$, and $T_{2}$ so that the open-loop transfer function $\mathrm{G}(\mathrm{s})$ meets equation (2.2). In other words, the parameters of the human operator transfer function, $H(s)$, 
depend strongly on the frequency spectrum of the forcing function of the open-loop, (which is the error between the desired output and the actual one), the gain $\mathbf{k}$ and the spectral properties of the controlled element $H_{c}$. Reference [39] shows that the open loop gain will decrease as the bandwidth of the external input $r(t), W_{r}$ increases so that their cross product, $|G(s)| W_{r}$, remains approximately constant in the cross-over region. Table (2.1) shows some of human operator's adaptation to the dynamics of controlled systems $[12,50]$.

TABLE 2.1

HUMAN OPERATOR'S MODEL ADAPTATION TO PLANT DYNAMICS

\begin{tabular}{c|c|c}
\hline $\mathrm{Y}_{\mathrm{c}}$ & $\mathrm{H}(\mathrm{s})$ & Parameters in $(2.1)$ \\
\hline 1 & $\left(\mathrm{~W}_{\mathrm{c}} / \mathrm{s}\right) \mathrm{e}^{-\mathrm{s} \tau}$ & $\begin{array}{c}\mathrm{k}=\mathrm{w}_{\mathrm{c}} \mathrm{T}_{2}, \tau_{\mathrm{n}}=0, \mathrm{~T}_{1}=0, \\
\mathrm{~T}_{2}>1\end{array}$ \\
\hline $1 / \mathrm{s}$ & $\left(\mathrm{W}_{\mathrm{c}}\right) \mathrm{e}^{-\mathrm{s} \tau}$ & $\mathrm{k}=\mathrm{w}_{\mathrm{c}}, \mathrm{T} 1=0, \mathrm{~T} 2=0$, \\
& & $\tau_{\mathrm{n}}=0$ \\
\hline $1 / \mathrm{s}^{2}$ & $\left(\mathrm{sw}_{\mathrm{c}}\right) \mathrm{e}^{-\mathrm{s} \tau}$ & $\mathrm{k}=\mathrm{W}_{\mathrm{c}} / \mathrm{T}_{1}, \mathrm{~T}_{1}>>1, \mathrm{~T} 2=0$, \\
& & $\tau_{\mathrm{n}}=0$ \\
\hline
\end{tabular}


At low frequencies, the experimental data show a residual phase lag which is not considered by equation (2.1) [52]. McRuer et. al. in 1965 [16] modified the equation (2.1), as formulated in equation (2.3), in order to have a better fit to the experimental data.

$$
H(s)=K \frac{1+T_{1} s}{1+T_{2} s} e^{-s\left(\tau_{\varepsilon}+\frac{\alpha}{\omega^{2}}\right)}
$$

The quantity $\alpha$ denotes a residual low frequency phase lead-lag coefficient. This phase is contributed by the term $\mathrm{e}^{-\left(\mathrm{s} \alpha / \mathrm{w}^{2}\right)}$.

It was shown that the modified model, Equation (2.3), becomes more useful when the human operator is monitoring a control system which is unstable and possesses a small phase margin [52].

In summary, the Cross-Over model allows us to predict the performance of manually-controlled systems, given knowledge of the system, $H_{c}$, the external input $r(t)$, and the remnant model, $n(t)$, which represents the degree of accuracy of the prediction. The CrossOver model for the human operator will not work if $H_{c}$ is sharply nonlinear, $H_{c} \sim K / s^{3}$, or if the bandwidth of $r(t), W_{r} \gg 10 \mathrm{rad} / \mathrm{sec}$ [16]. For more about the Cross-Over model, its application and deficiencies, and new modified approaches, one may refer to [50,52-55].

\subsubsection{Optimal Control Model (OCM)}

As mentioned earlier, there are two predominant human operator performance modeling approaches: structural and algorithmic models [41]. The structural approach is mainly based on 
applicable to the manual control of single input/output linear time invariant systems.

The algorithmic model is based on linear quadratic Gaussian state-space optimal control theory, which is modified for acceptance of the human operator's inherent limitations. In other words, the OCM applies modern control techniques, state space analysis, optimization and estimation, in order to maximally use the adaptive characteristics of the human operator subject to his constraints, which are represented via the visual and motor noises and timeconstant delays [41,49]. Because this study uses the OCM model extensively, the theory of optimal control and the OCM are considered in chapters III and IV respectively.

\subsection{Summary}

In this chapter we briefly reviewed the behavior of the human operator, as well as the characteristics of the models which may be used to describe and predict the human performance in manuallycontrolled systems. The characteristics of manual control systems are discussed in section 2.2. The operation of human operator, as a controller or information processor, in a simple manual control system has been considered in section 2.3. Analysis methods for measuring human operator performance have been introduced in section 2.4 .

There are two powerful models for the human operator in manual control systems: the Cross-Over model and the optimal control model. The Cross-Over model, as a good predictive tool, is 
only applicable to the manual control of single-input/single-output linear time invariant systems. Section 2.4 .2 discussed the Cross-Over model in detail and section 2.4 .3 briefly introduced the optimal control model, which can be applied to both single-input/singleoutput and multivariable linear manual control systems. 


\section{CHAPTER III}

\section{OPTIMAL CONTROL}

\subsection{Introduction}

Since 1868, when Maxwell made the first mathematical model for stability analysis, till now, control theory has made significant progress. Frequency domain techniques in the 1930s, Laplace transforms (Transfer functions) in the 1940s, optimal control in the late 1950s and state-space analysis (a time-domain approach) in the 1960s made the progress possible [56-58]. These versatile methods may be rooted in classical control theory or modern control theory.

Classical control design, a trial \& error approach, applies an iterative analysis method in order to determine the design parameters, satisfying some time/frequency domain criteria such as settling time, maximum overshoot, gain and phase margin. However, in practice, there are many control problems such as airplane landing problems [59], which can only be analyzed if some multiple performance requirements and constraints are satisfied, specifically, when the performance tasks require a time-varying control whose configuration and values of time-varying parameters must be selected by the designers. In such cases, a synthesis method based on optimization theory is superior to classical trial and error techniques, since the selection of the time-varying gains is basically replaced by 
the selection of constant parameters used as weighting factors in the performance index [60].

Therefore, there was an obligatory shift toward optimal control design of more realistic and complex systems having a large number of variables including input/output variables and containing nonlinearities and time-varying parameters. Modern control theory using state space analysis provides a powerful methodology for dealing with these high order, linear or nonlinear multivariable systems. However, classical techniques are restricted to almost linear constant single input/output systems [56].

In order to control a process, we need to know the current state of the process. Controlling a process means to find a way in which to manipulate the input so that the system will behave in some prescribed way. In most practical cases, the current state of the system is unknown. Therefore, we need to estimate the current state from a set of observed measurements. Here, estimation and control theory come together. Figure 3.1 shows the general framework of estimation and control. In the this figure, $\underline{\underline{u}}(t)$ is a manipulator input, $\underline{w}(t)$ is a disturbance input and $\underline{v}(t)$ is measurement noise.

The physical element (plant) is affected by a disturbance input $\underline{w}(t)$ which represents some unmanipulated inherent limitations of the plant and its environment, such as noise in circuits and wind gusts in aircraft flights. We may observe the behavior of the plant by using some appropriate devices, sensors, which are subjected to some random errors represented by $\underline{v}(t)$. Consequently, the observed outputs of the system will give some uncertain information about the controlled process (plant). This requires us to estimate the system 
response variables, such as the system states, in order to effectively control the plant. The estimation can be done by determining an approximation to the states from the measurements. The relative goodness of the estimates is measured by some performance indices. An estimate is optimal if it is obtained by optimizing some performance criteria.

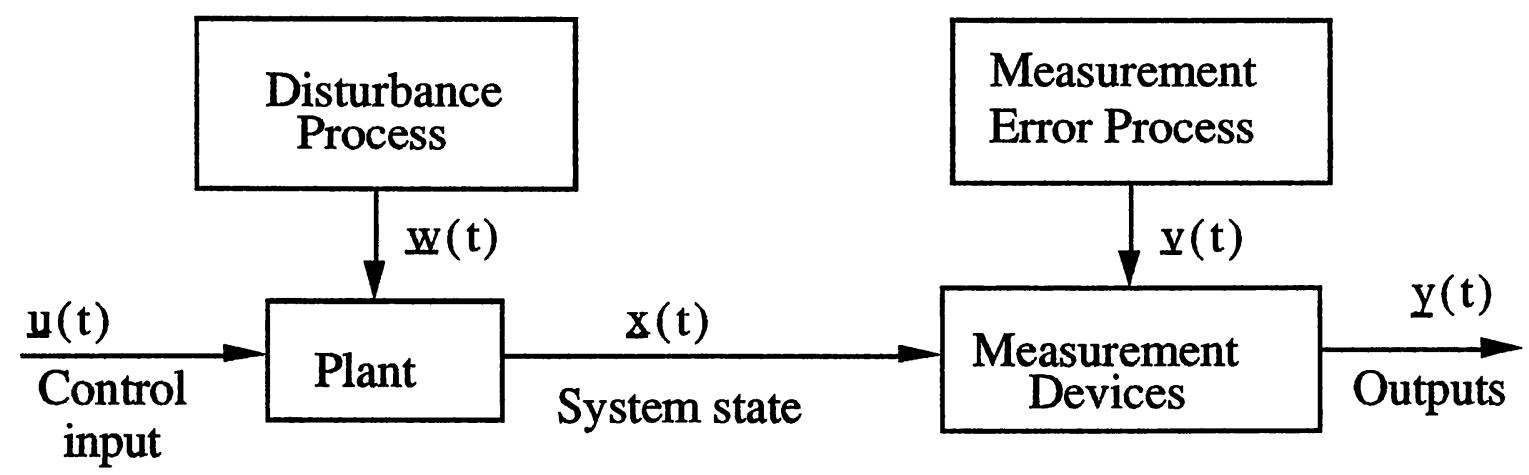

Figure 3.1. General Scheme of Estimation \& Control

Figure 3.1 represents also a general stochastic control problem [63-64]. The optimal control for this problem is defined as follows: knowing the plant and the statistics of the unwanted noises $\underline{w}(t)$ and $\underline{v}(t)$, we wish to determine a control input $\underline{u}^{*}(t)$ which minimizes a specified performance criterion so that the overall system performs in an optimal fashion. To do so, we first need to determine an optimal estimate of the state from a set of measurements and then to find an optimal control input which would be a function of the state estimate, 
so that the overall system performance agrees with some desired objectives.

The linear time-invariant stochastic optimal problem is the major concern in this study. The rest of this chapter is organized as follows. Sections 3.2-3.4 discuss state variable models, linear systems and linear optimal controllers, respectively. Section 3.5 considers regulator problems. State estimation problems are discussed in section 3.6. The separation theorem and the solution of the optimal controller for stochastic linear systems are presented in section 3.7. Tracking problems are discussed in section 3.8. Finally, section 3.9 summarizes this chapter.

\subsection{State Variables}

State variable analysis, since the 1960 s, has become a powerful approach to deal with multivariable (multi-input/multi-output) control system problems. A set of state variables of a system is defined as a minimum independent set of parameters, $\underline{x}(t)$, being able to show the complete status of the underlying system in the sense that the output of the system, $y(t)$, and the values of $\underline{x}(t)$ can be uniquely determined by knowing the values of $\underline{x}\left(t_{0}\right), t_{0}<t$, provided that control input $\underline{\mathrm{u}}(\tau), \underline{\mathrm{u}}(\tau), \mathrm{t}_{0} \leq \tau \leq \mathrm{t}$ is known. Figure 3.2 shows a simple black-box representation of a physical system.

The definition of the state variables leads to the following transformation on the input, state and output.

$$
\begin{aligned}
& \underline{\mathrm{x}}(\mathrm{t})=\underline{\Psi}\left(\mathrm{t}_{0}, \mathrm{t}, \underline{\mathrm{x}}\left(\mathrm{t}_{0}\right), \underline{\mathrm{u}}(\tau), \quad \tau \in\left[\mathrm{t}_{0}, \mathrm{t}\right]\right) \\
& \underline{\mathrm{y}}(\mathrm{t})=\underline{\mathrm{h}}(\mathrm{t}, \underline{\mathrm{x}}(\mathrm{t}), \underline{\mathrm{u}}(\mathrm{t}))
\end{aligned}
$$


The functions $\underline{\Psi}$ and $\underline{\hbar}$ must have the following three properties.

i) $\quad \underline{\mathrm{x}}\left(\mathrm{t}_{0}\right)=\underline{\Psi}\left(\mathrm{t}_{0}, \mathrm{t}_{0}, \underline{\mathrm{x}}\left(\mathrm{t}_{0}\right), \underline{\mathrm{u}}\left(\mathrm{t}_{0}\right)\right)$

ii) If $\underline{\mathrm{u}}(\tau)=\underline{\mathrm{v}}(\tau), \mathrm{t}_{0} \leq \tau \leq \mathrm{t}_{1}$, then

$$
\psi\left(\mathrm{t}_{0}, \mathrm{t}_{1}, \underline{\mathrm{x}}\left(\mathrm{t}_{0}\right), \underline{\mathrm{u}}(\tau)\right)=\psi\left(\mathrm{t}_{0}, \mathrm{t}_{1}, \underline{\mathrm{x}}\left(\mathrm{t}_{0}\right), \underline{\mathrm{v}}(\tau)\right)
$$

iii) $\underline{\mathrm{x}}\left(\mathrm{t}_{2}\right)=\psi\left(\mathrm{t}_{0}, \mathrm{t}_{2}, \underline{\mathrm{x}}\left(\mathrm{t}_{0}\right), \underline{\mathrm{u}}(\tau), \quad \tau \in\left[\mathrm{t}_{0}, \mathrm{t}_{2}\right]\right)$

$$
=\psi\left(\mathrm{t}_{1}, \mathrm{t}_{2}, \underline{\mathrm{x}}\left(\mathrm{t}_{1}\right), \underline{\mathrm{u}}(\tau), \tau \in\left[\mathrm{t}_{1}, \mathrm{t}_{2}\right]\right)
$$

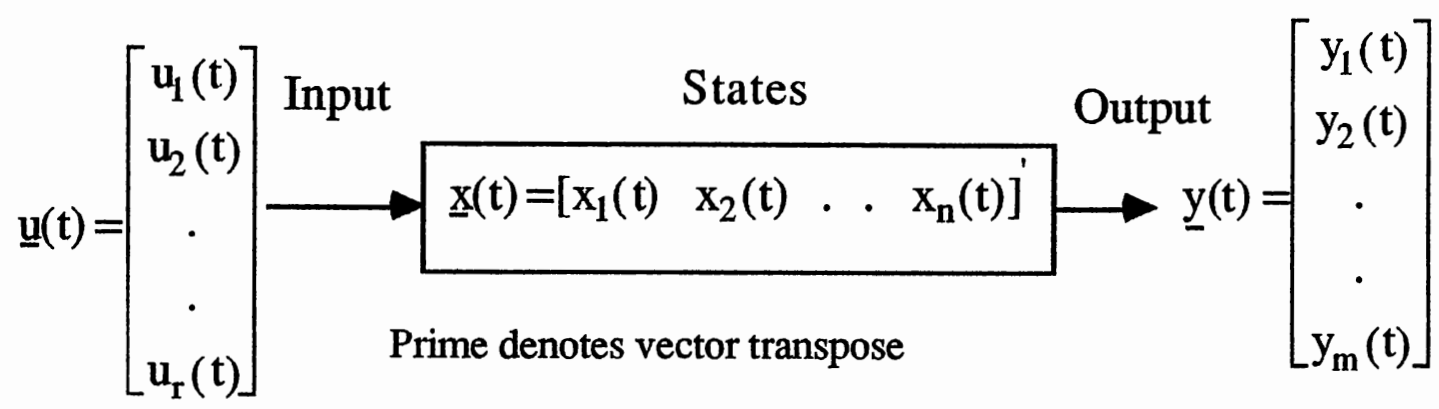

Figure 3.2. A simple black-box diagram of a physical system

For a continuous time-variant dynamic system, equation (3.1) may be replaced by a system of first order ordinary differential equations of the form

$$
\underline{\dot{x}}(t)=\underline{f}(\underline{x}, \underline{u}, t) .
$$

There is a unique solution for equation (3.6) if

i) $\underline{u}(t)$ is piecewise continuous

ii) $\underline{\mathbf{f}}$ is continuous with respect to $u$.

iii) $\underline{f}$ is piecewise continuous with respect to $t$. and 
iv) $\|\underline{f}(\underline{\mathrm{x}}, \mathrm{t})-\underline{\mathrm{f}}(\underline{\mathrm{z}}, \mathrm{t})\| \leq \mathrm{C}\|\underline{\mathrm{x}}-\underline{\mathrm{z}}\|$, for some constant $\mathrm{C}>0$.

This solution can be expressed by equation (3.1) $[56,57,61,62]$.

\subsection{Linear Systems}

In practice, the functions $\underline{f}$ and $\underline{h}$ in equations (3.6) and (3.2) are nonlinear. In general, the operation of nonlinear time-varying dynamic system may be described by the following equations

$$
\begin{aligned}
& \underline{\dot{x}}(t)=\underline{f}(\underline{x}, \underline{u}, \underline{w}, t) \\
& \underline{y}(t)=\underline{h}(\underline{x}, \underline{u}, \underline{w}, \underline{v}, t)
\end{aligned}
$$

Where $\underline{w}(t)$ and $\underline{v}(t)$ are state disturbances and measurement noises respectively. Equation (3.7) represents an n-component vector of first order differential equations describing the dynamics of the system and equation (3.8) represents the measurements (observed outputs of the system). Unfortunately, there is no complete analytical solution to equation (3.7), Whereas, there is a general methodology to solve a set of linear differential equations. Consequently, linear dynamical systems became very attractive. The following equations may represent the general state space form of a linear time-variant continuous time dynamic system.

$$
\begin{aligned}
& \underline{\dot{x}}(t)=A(t) \underline{x}(t)+B(t) \underline{u}(t)+E(t) \underline{w}(t) \\
& \underline{y}(t)=C(t) \underline{x}(t)+D(t) \underline{u}(t)+\underline{v}(t)
\end{aligned}
$$

Where

$$
\begin{aligned}
& \underline{\underline{x}}(t)-\text { an } n \text {-component state vector } \\
& \underline{\mathbf{u}}(\mathrm{t})-\text { an } r \text {-component control input vector }
\end{aligned}
$$


$\mathrm{y}(\mathrm{t})$ - an m-component output vector

$\underline{w}(t)$ a $p$-component state noise vector

$\underline{\mathrm{v}}(\mathrm{t})$ - an m-component measurement noise vector

and the compatible matrices $\mathrm{A}, \mathrm{B}, \mathrm{C}, \mathrm{D}$ and $\mathrm{E}$ are continuous.

The linear system is called fixed or time-invariant if the matrices A, B, C, D, and E do not change with time. Figure 3.3 illustrates the general block-diagram for a continuous linear timeinvariant stochastic system.

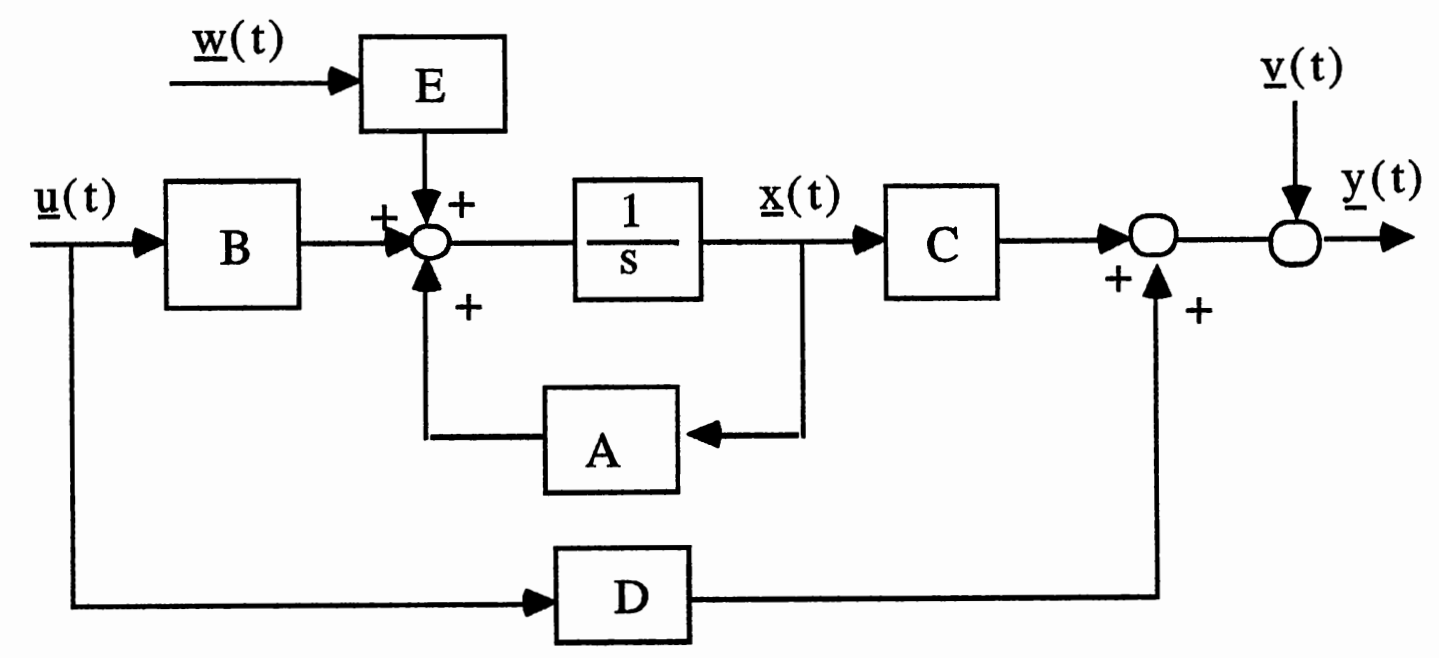

Figure 3.3. Block-Diagram for a continuous Linear Time-Invariant Stochastic System

This study considers systems illustrated in Figure 3.3. In order to deal with these systems we need to know the statistical characteristics of both noises $\underline{w}(t)$ and $\underline{v}(t)$. However, we assume that we can observe only the measurements $y(t)$ and we have access only 
to the control input $\underline{u}(t)$. In other words, by observing $y(t)$, we are trying to determine the characteristics of the system and by manipulating $\underline{u}(t)$, we wish to force the system to react in some desired manner.

\subsection{Linear Optimal Controller}

For linear optimal control, the device which produces the optimum control input, is linear, i.e. the optimal control law is a (constant) linear combination of the state variables. We shall see later in this chapter that the linear optimal controller is attainable by selecting a performance index which is a quadratic function of the motions of the system and inputs.

Mathematically, the problem is stated as follows: For the given linear time-invariant system

$$
\begin{aligned}
& \underline{\dot{x}}(t)=A \underline{x}(t)+B \underline{u}(t) \\
& \underline{y}(t)=C \underline{x}(t)
\end{aligned}
$$

and for some initial state $\underline{x}(0)$ find a control input, $\underline{u}^{*}(t)$, that minimizes the quadratic performance index

$$
J=\frac{1}{2} \int_{0}^{\infty}\left(\underline{x^{\prime}} \underline{Q} \underline{x}+\underline{u^{\prime}} \mathbf{R} \underline{u}\right) d t
$$

where $R$ is a positive definite symmetric matrix, $Q$ is a nonnegative definite symmetric matrix and prime denotes matrix transpose. 
The cost function $J$ is a scalar quantity which consists of an infinite integral of sums of quadratic functions of the output and the control inputs to the system (3.11). This quadratic form of the cost ensures the linearity of the optimal control and the constraints on weighting matrices $Q$ and $R$ insure a finite control law. The terms in the performance index $\mathrm{J}$, must represent the desirable quality of the overall system performance. For example, if we are able to predict the closed loop eigenstructure of the system, we will be capable of selecting right weighting factors $Q$ and $R$ [58,65-67].

An intuitive reason for using linear quadratic methods may be stated as: Wonham [67] showed that if the open-loop system (3.11) is completely controllable, we can assign a conjugate set of desirable numbers to the closed loop poles of the system by an appropriate state feedback gain. This gain matrix is not unique for multivariable systems. There is no restriction on choosing these prespecified set of closed loop poles. The more the closed loop poles move to the left in Splane, the faster states of the system converge and consequently, the more input amplitude (control energy) is required. But in any realizable system a bounded input is required. This requires that the poles not be moved beyond some certain distance to the left in the complex plane. Therefore, it is necessary to consider both the rate of convergence and the magnitude of the input. This may be done by choosing a performance criterion like $\mathrm{J}$ in Equation (3.12).

Several advantages of the linear optimal control have been enumerated in the literature [60]. Here, we list some of them and end this section. 
1. Linear optimal control system analysis is simpler than the conventional trial \& error method.

2. All linear optimal control problems have computable solutions. This is not true for non-linear optimal control problems.

3. It is possible to generalize the linear optimal control problem to include a performance index involving any quadratic form of the output and its derivative.

4. It is possible to apply linear optimal control to non-linear systems.

5. The linear optimal control guarantees a stable closed loop system with a smooth and well-behaved transient response.

\subsection{Noise Free Regulator Problems}

\subsubsection{Problem Statement}

Here, we consider the following noise free plant (3.9)

$$
\underline{\dot{x}}(t)=A(t) \underline{x}(t)+B(t) \underline{u}(t)
$$

We wish to move the system (3.13) from a non-zero initial state, $\underline{x}\left(t_{0}\right)$ to the zero terminal state, $\underline{x}(T)=0$ by applying some acceptable input amplitude, $\underline{u}(t)$. To do this, we may define the following quadratic performance index with quadratic form in the terminal state plus an integral of quadratic terms in the state and the control input

$$
J=\frac{1}{2} \int_{t_{0}}^{T}\left(\underline{x}^{\prime}(t) Q(t) \underline{x}(t)+\underline{u}^{\prime}(t) R(t) \underline{u}(t)\right) d t+\underline{x}^{\prime}(T) S \underline{x}(T),
$$

where the matrices $Q(t)$ and $R(t)$ have continuous entries. They are symmetric non-negative and positive definite, respectively. 
The scalar quantity $\mathbf{J}$ measures the quality of the overall system. The term $\underline{x}^{\prime} Q \underline{x}$ in equation (3.14) is a measure of the distance of the state $\underline{x}(t)$ from the zero state, and the weighting matrix $Q$ determines the amount of the weight assigned to each of the components of the states. The quantity $\int_{t}^{T} \underline{x}^{\prime}(t) Q(t) \underline{x}(t)$ determines the cumulative deviations of the state at time $t, \underline{x}(t)$ from the zero state during the interval $[t, T]$. The same is true for the other terms in (3.14).

Before presenting the solution of the regulator problem, let us have a brief qualitative description of the regulator problem. Figure 3.4 shows a general control problem which has the following key elements:

i) The plant for which a control input $u(t)$ is desired.

ii) The measurement devices which give information about the plant.

iii) The controller which produces the proper input to the plant. To do that, controller uses the the reference input, i.e. desired output, as a base for comparison with the observed data.

In general, the controller is expected to generate an input $u(t)$ to achieve the following goal: For a given reference input $r(t)$, determine a proper input $u^{*}(t)$ such that the controlled variable $y(t)$, tracks $r(t)$. This is called a tracking problem. A special class of tracking problems is called the regulator problem, where the reference input, $r(t)$, is a constant function. In other words, the regulator problem is to find a control input to bring the plant from a non-zero state to a constant, generally zero, state. 


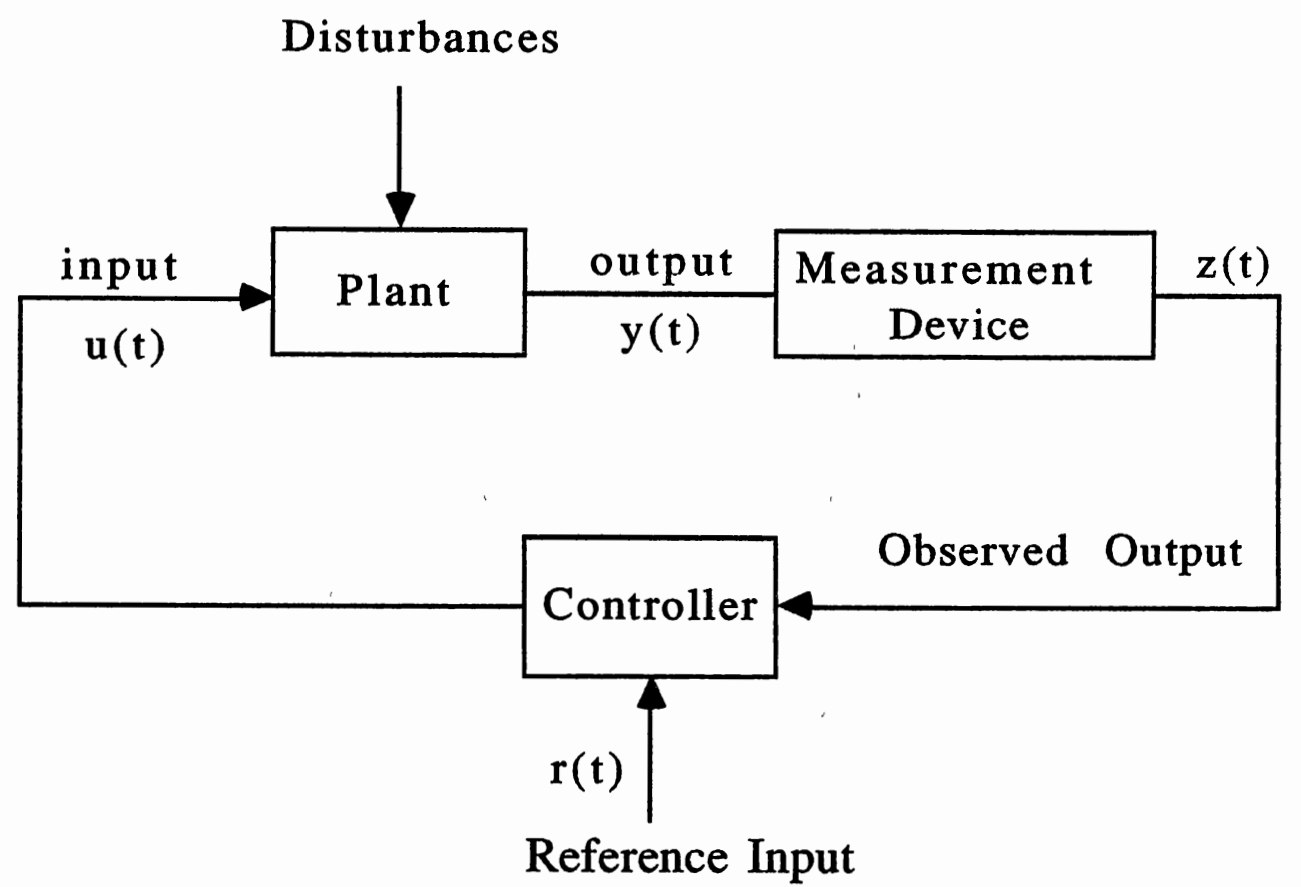

Figure 3.4. General Representation Of A Control Problem

In short, the regulator is designed to keep a stationary plant subjected to some unwanted disturbances as close as possible to the zero state by using the smallest possible amounts of control input.

\subsubsection{Solution of the Noise-Free}

Regulator Problem

The regulator problem, as a special class of optimization problem, can be solved by applying the calculus of variation technique [67] or by using dynamic programming [60]. The analytical solution may be formulated as:

$$
\underline{\underline{u}} *(t)=-F(t) \underline{x}(t)
$$


with the controller gain matrix

$$
F(t)=R^{-1}(t) B^{\prime}(t) P(t)
$$

where the positive definite symmetric matrix $p(t)$ is the solution of the Riccati equation

$$
\begin{aligned}
& \dot{P}(t)+P(t) A(t)+A^{\prime}(t) P(t)- \\
& P(t) B(t) R^{-1}(t) B^{\prime}(t) P(t)+Q(t)=0
\end{aligned}
$$

with the boundary condition $P(T)=S$.

The solution of the optimal noise-free linear regulator problem is in the form of a linear control law, see Equation (3.15), which produces the optimal control for any initial state, $\underline{x}(t)$. Figure 3.5 shows a block-diagram of the noise-free linear regulator controller.

The optimal performance index, $J_{o p t}(\underline{x}, t)$ exists for all $t$ less than or equal $\mathrm{T}$ and has the following quadratic form

$$
J_{\text {opt }}(\underline{x}, t)=\underline{x}^{\prime}(t) P(t) \underline{x}(t)
$$

where $P(t)$ satisfies the Riccati equation (3.17).

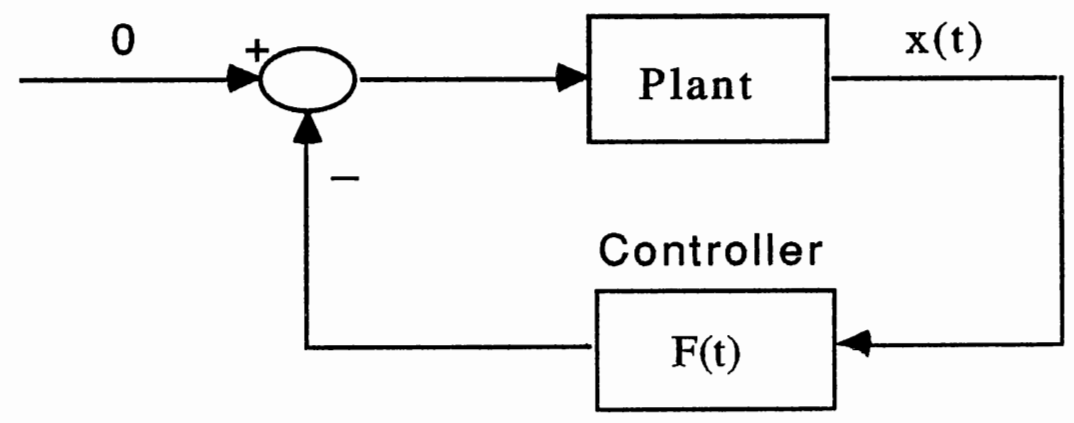

Figure 3.5. Noise-Free Linear Regulator Controller 
If $T$, the upper limit of the integral in the performance index $J$ (equation (3.14)), approaches infinity, then the problem is called infinite-time noise-free regulator. It is of practical importance to know whether the time-invariant plants (3.13) will lead to a constant controller law or not. For finite-time regulator problems, the optimal performance index, Jopt is always finite, but this is not true for infinite regulator problems unless the time-invariant system (3.13) is completely controllable or the uncontrollable states are stable [47]. In this case, the performance index (3.12) will be of the form

$$
J=\frac{1}{2} \int_{t_{0}}^{\infty}\left(\underline{x}^{\prime}(t) Q \underline{x}(t)+\underline{u}^{\prime}(t) R \underline{u}(t)\right) d t
$$

since for noise free system (3.13), the terminal state will be zero,

$$
\lim _{t \rightarrow \infty} x(t)=0 \text {. }
$$

The solution to the infinite regulator problem gives rise to the linear time-invariant optimal control law

$$
\underline{\underline{u}} *(t)=-F \underline{x}(t)
$$

where the constant matrix $F$ is the optimal controller gain matrix

$$
\mathbf{F}=\mathbf{R}^{-1} \mathbf{B}^{\prime} \mathbf{P}_{\mathrm{s}}
$$

and $P_{s}$ is the solution of the Riccati equation

$$
\mathrm{A}^{\prime} \mathbf{P}_{\mathrm{s}}+\mathrm{P}_{\mathrm{s}} \mathrm{A}+\mathrm{P}_{\mathrm{s}} \mathrm{B} \mathrm{R}^{-1} \mathrm{~B}^{\prime} \mathrm{P}_{\mathrm{s}}+\mathrm{Q}=0
$$


with the optimal index

$$
J_{o p t}(x, t)=\underline{x}^{\prime}(t) P_{s} \underline{x}(t)
$$

Now, the question is raised whether the overall closed-loop system formed with the constant control law, equation (3.20), is asymptotically stable or not. In other words, when is the closed-loop system

$$
\underline{\dot{x}}(t)=(A-B F) \underline{x}(t)
$$

stable? It has been shown that $[60,67]$, in order to ensure the asymptotically stability of Equation (3.24), the pair [A,D] must be detectable for any $\mathrm{D}$ such that $\mathrm{Q}=\mathrm{DD}$ '.

In summary, for linear time-invariant noise-free finite dimensional systems, the infinite-time regulator problem possesses a constant linear optimal control law which gives an asymptotically stable closed-loop system if the system is completely stabilizable and detectable. Furthermore, one may modify the performance index (3.19) for the time-invariant system (3.13) by introducing a weighting term, $e^{\alpha t}$, in order to achieve a prespecified $\alpha$-degree of stability for the closed-loop system (3.24) [60].

\subsection{State Estimation Problem}

The preceding section considered the regulator problem based on the unrealistic assumption that the states of the controlled system are completely and accurately measurable, moreover it has been 
shown that the optimal control laws are dependent on the system states. In practical situations, we do not have access to the states completely. For example, for most of the linear systems, only certain linear combinations of the states, output variables, can be measured. Therefore, the problem of estimating or reconstructing the states of a dynamical system given the output measurements is of fundamental importance.

For linear systems, there are two famous approaches. If there is no disturbance acting on the system states, a Luenberger deterministic state estimator, state observer, can be applied to reconstruct the states. For stochastic linear system modeling, where the output variable are corrupted by noise (white), the statistical state estimator design (Kalman-Bucy Filter) can be used to reconstruct the states of the system. The latter is an optimal state estimator in the sense that the noise has the minimum possible effect when the estimator is used in controlling a plant.

Figure 3.6 shows a general scheme of using an estimator in implementing a linear dynamic system. In general, a state estimator which (is actually a model for the plant) is a dynamical system whose inputs are the plant inputs and outputs and its output is the current estimate of the system state. Because, in this study, the linear stochastic systems are our major concern, we consider the Kalman Filter (optimal state estimator) in the next section. 


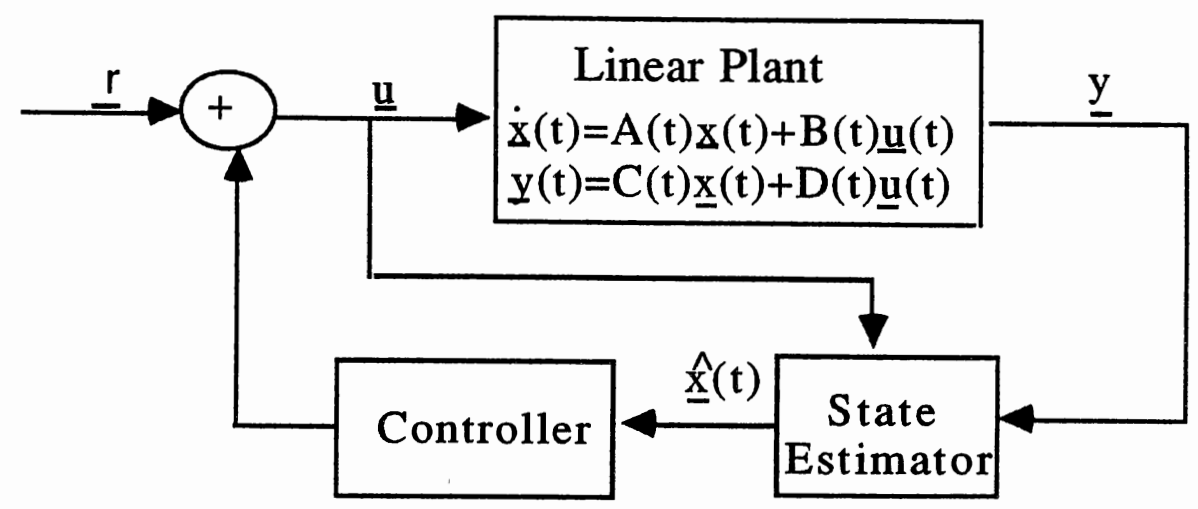

Figure 3.6. Using estimator in control law

\subsubsection{Optimal State Estimator}

Figure 3.7 illustrates the structure of the optimal estimator. The plant is of the general form given in (3.9) which we repeat below.

$$
\begin{aligned}
& \underline{\dot{x}}(t)=A(t) \underline{x}(t)+B(t) \underline{u}(t)+E(t) \underline{w}(t) \\
& \underline{y}(t)=C(t) \underline{x}(t)+D(t) \underline{u}(t)+\underline{v}(t)
\end{aligned}
$$

where

$\underline{w}(t)$ - n-component state excitation white noise with intensity $\mathrm{W}(\mathrm{t}), \mathrm{E}\left[\underline{\mathrm{w}}(\mathrm{t}) \underline{w}^{\prime}(\tau)\right]=\mathrm{W}(\mathrm{t}) \delta(\mathrm{t}-\tau)$,

$\underline{v}(t)$ - m-component observation error white noise vector with intensity $V(t), E\left[\underline{v}(t) \underline{y}^{\prime}(\tau)\right]=V(t) \delta(t-\tau)$.

It is assumed that $\mathrm{V}(\mathrm{t})$, for all $\mathrm{t}$, is a positive definite symmetric matrix. It means that we can not extract any noise-free information from the measurements; all components of $\mathrm{y}(\mathrm{t})$ are corrupted by 
noise. Further, it is assumed that $\underline{w}(t), \underline{v}(t)$ and $\underline{x}(t 0)$ are independent and Gaussian with

$$
\begin{aligned}
& E[\underline{v}(t)]=E[\underline{w}(t)]=0, E\left[\underline{x}\left(t_{0}\right)\right]=\underline{x}_{0} \\
& E\left[\left(\underline{x}\left(t_{0}\right)-\underline{x}_{0}\right)\left(\underline{x}\left(t_{0}\right)-\underline{x}_{0}\right)^{\prime}\right]=P_{0} \\
& E\left[\underline{x}\left(t_{0}\right) \underline{v}^{\prime}(t)\right]=E\left[\underline{x}\left(t_{0}\right) \underline{w}^{\prime}(t)\right]=0 \text { for all } t
\end{aligned}
$$

and $W(t)$ is nonnegative definite symmetric matrix.

The task of estimation may be stated as follows: knowing the statistical characteristics of $\underline{x}\left(t_{0}\right), \underline{w}(t)$ and $\underline{v}(t)$, we wish to use the available information of the plant input, $\underline{\mathbf{u}}(\tau)$, and plant output, $y(\tau)$ for $\tau$ between $t_{0}$ and $t$, to obtain an optimal estimate, $\underline{\hat{x}}(t)$ of the state, $\underline{x}(t)$, which minimizes the following error variance performance index

$$
J=E\left[(\underline{x}(t)-\underline{\hat{x}}(t))^{\prime} \Omega(t)(\underline{x}(t)-\underline{\hat{x}}(t))\right]
$$

where $\Omega(t)$ is a positive definite symmetric weighting matrix

The state estimator models the plant with the dynamical equation of the form

$$
\underline{\hat{\mathbf{x}}}(\mathrm{t})=\mathrm{A}(\mathrm{t}) \underline{\hat{\mathrm{x}}}(\mathrm{t})+\mathrm{B}(\mathrm{t}) \underline{\mathrm{u}}(\mathrm{t})+\mathrm{K}(\mathrm{t}) \mathrm{C}(\mathrm{t})[\underline{\mathrm{x}}(\mathrm{t})-\underline{\hat{\mathrm{x}}}(\mathrm{t})]
$$

where $K(t)$ is the estimator gain matrix which will be given in the next section. Figure 3.7 shows the block-diagram of the state estimator formulated in (3.27). 
By subtracting (3.27) from (3.9a), we can have the following dynamical equation

$$
\underline{\dot{e}}(t)=[A(t)-K(t) C(t)] \underline{e}(t)+E(t) \underline{w}(t)
$$

where $\underline{e}(t)=\underline{x}(t)-\underline{\hat{x}}(t)$ is the estimation error. Equation (3.28) shows that

i) if $\underline{x}\left(t_{0}\right)=\underline{\hat{x}}\left(t_{0}\right)$, for any initial time $t_{0}$, then $\underline{x}(t)=\underline{\hat{x}}(t)$ for all $t \geq t_{0}$ and for all input $\underline{u}(t)$ provided that $\underline{w}(t)=0$.

ii) $\quad \lim _{t \rightarrow \infty} \underline{e}(t)=0$ iff $A_{0}(t)=A(t)-K(t) C(t)$ is asymptotically stable.

Because we wish the estimation error to approach zero, the right selection for the estimator gain matrix $K(t)$ is of fundamental importance. The right choice of $\mathrm{K}(\mathrm{t})$ affects the rate of convergence $\underline{\hat{x}}(t)$ to $\underline{x}(t)$.

\subsubsection{Solution of the Optimal Estimator Problem}

It can be shown [60] that in order to obtain an optimal estimator, $\underline{\hat{x}}(t)$, we need to choose the following estimator gain matrix

$$
K^{*}(t)=P(t) C^{\prime}(t) V^{-1}(t), t>t_{0}
$$

Where $P(t)$ is the error covariance matrix, $P(t)=E\left[\underline{e}^{\prime} \underline{e}\right]$, and it is determined by solving the following matrix Riccati equation

$$
\begin{aligned}
\dot{P}(t)= & A(t) P(t)+P(t) A^{\prime}(t)+E(t) W(t) E^{\prime}(t) \\
& -P(t) C^{\prime}(t) V^{-1}(t) C(t) P(t),
\end{aligned}
$$


with the initial boundary condition $P\left(t_{0}\right)=P_{0}$. While the mean square estimation error is

$$
E\left[\underline{e}^{\prime}(t) \Omega(t) \underline{e}(t)\right]=\operatorname{Tr}\{P(t) \Omega(t)\}
$$

where $\operatorname{Tr}($.$) denotes trace(.) and \underline{e}(t)=\underline{x}(t)-\underline{\hat{x}}(t)$.

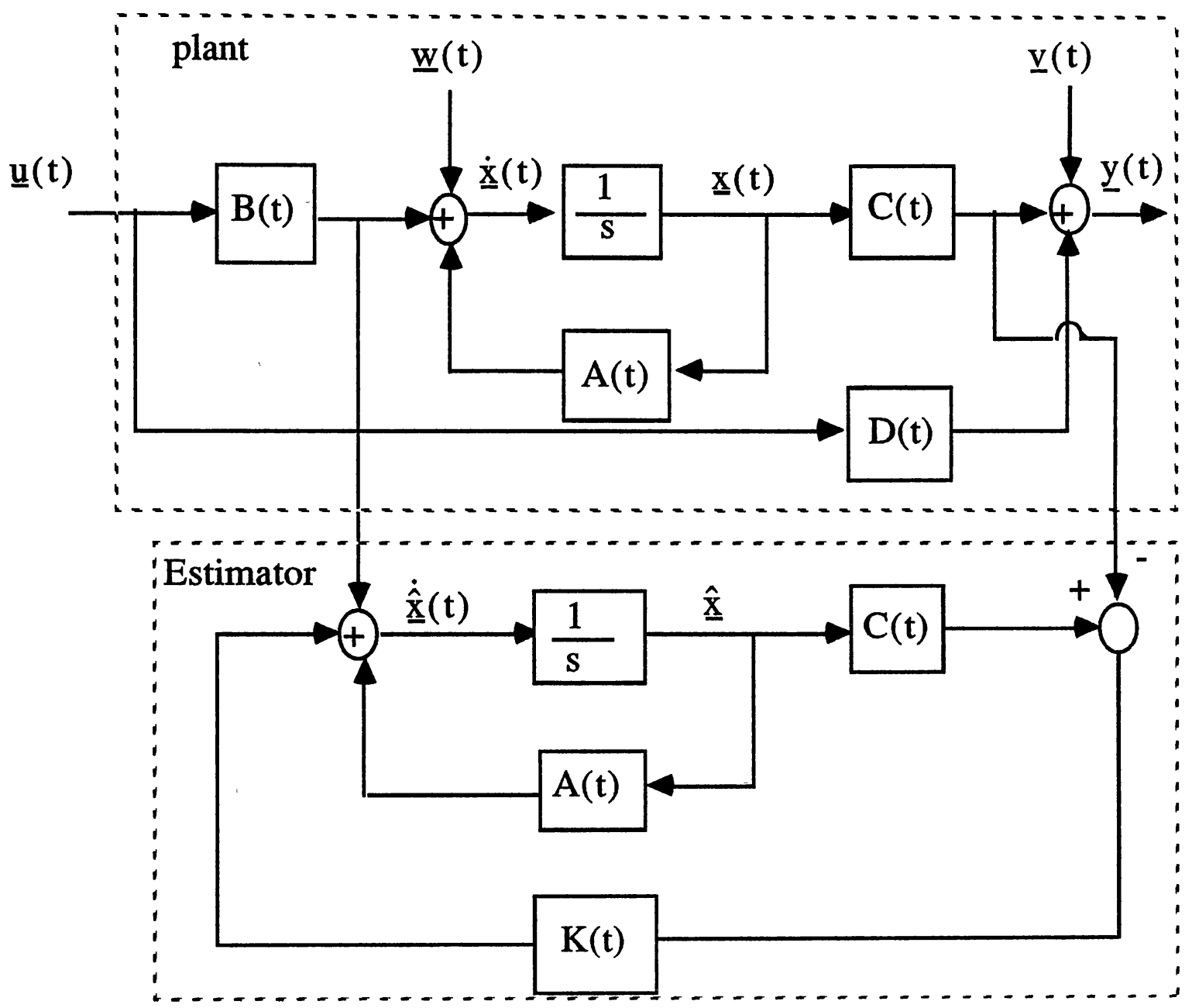

Figure 3.7. Plant-Estimator Structure For Linear Stochastic Systems 
The solution of the optimal estimator problem is independent of the reconstruction error weighting matrix $\Omega(t)$. This optimal estimator is known as the Kalman-Bucy filter. We are not able to find any other linear functional of the observations $y(\tau)$ and input $\underline{\mathbf{u}}(\tau)(\tau$ is in the range $\left.\left[t_{0}, t\right]\right)$ that produces an estimate of $\underline{x}(t)$ with a smaller mean square estimation error.

For the time-invariant plant (3.9), the following equations solve for the constant estimator gain provided that the time-invarint plant (3.9) is completely detectable.

$$
\mathrm{K}=-\overline{\mathrm{P}} \mathrm{CV} \mathbf{-}^{-1}
$$

where $\overline{\mathbf{P}}$ is the error covariance matrix satisfying the Riccati equation

$$
\overline{\mathrm{P}} \mathrm{A}^{\prime}+\mathrm{A} \overline{\mathrm{P}}+\mathrm{EWE} \mathrm{E}^{\prime}=\overline{\mathrm{P}} \mathrm{CV}^{-1} \mathrm{C}^{\prime} \overline{\mathrm{P}}
$$

and further, if the pair $[A, G]$ is stabilizable for any matrix $G$ such that $G^{\prime}=W$, then the optimal estimator is asymptotically stable, i.e. the eigenvalues of the matrix $A_{0}=A-K C$, have negative real parts.

Consequently, $\underline{e}(t)$ goes to zero at a certain exponential rate determined effectively by the choice of the estimator gain $K(\underline{\hat{x}}(t)$ will track $\underline{x}(t)$ after a finite period of time determined by the eigenvalues of the matrix $A_{0}$ ).

In short, the estimator problem is to find an optimal gain, $K^{*}(t)$, by minimizing equation (3.26) given (3.9), (3.27) and the statistical information about $\underline{x}\left(t_{0}\right), \underline{w}(t)$ and $\underline{v}(t)$ (for the time invariant case the noise intensities are constant). 


\subsection{Optimal Controller for Stochastic Linear Systems}

As discussed before, stochastic linear systems are defined by Equations (3.9) with the appropriate assumptions given in Equations (3.10) and (3.25). Stochastic plant models represent a tool for introducing a quantitative measure of the uncertainty of a system to the dynamic characteristics of the plant ( see Equation (3.9a) which represents a random dynamical equation). Therefore, the performance measure associated with any decision policy on the system (3.9) must be random. As we mentioned earlier, in order to find an optimal controller, we need to select an appropriate scalar valued cost functional. Since the system behavior is random, it is unrealistic to choose a performance index as we had before, Eq.(3.14).

Because of its analytical tractability, the mean utility of the performance index, equation (3.32) is the most commonly used performance criterion in linear stochastic systems.

$$
J=E\left\{\underline{x}^{\prime}(T) S \underline{x}(T)+\int_{t_{0}}^{T}\left(\underline{x}^{\prime}(t) Q(t) \underline{x}(t)+\underline{u}^{\prime}(t) R(t) \underline{u}(t)\right) d t\right\}
$$

where $\mathrm{S}$ is a symmetric $\mathbf{n}$ by $\mathbf{n}$ nonnegative definite matrix, $Q(t)$ is a symmetric continuous $n$ by $n$ nonnegative definite matrix, $R(t)$ is a symmetric continuous $\mathrm{r}$ by $\mathrm{r}$ positive definite matrix and $\mathrm{E}$ denotes the expected value over $\underline{x}\left(t_{0}\right), \underline{v}(\tau)$ and $\underline{w}(\tau)$ on the whole operation time interval $\left[\mathrm{t}_{0}, \mathrm{~T}\right]$.

The performance index $\mathrm{J}$ is quadratic in the state and the control. The first term in $J, \underline{x}^{\prime}(T) S \underline{x}(T)$, is a measure of the terminal 
error, while the integral term indicates the system error and control effort over the entire time interval $\left[\mathrm{t}_{0}, \mathrm{~T}\right]$. Here, we require that the control input at time $t$ be realizable in terms of the system outputs $\mathrm{y}(\tau)$, which are physically available for $\tau$ in $\left[\mathrm{t}_{0}, \mathrm{~T}\right]$, and also in terms of the initial statistics of $\underline{x}\left(t_{0}\right)$. Therefore, the control law has to have the form

$$
\underline{u}(t)=f\left[\underline{y}(\tau), t_{0} \leq \tau \leq t, \underline{x}_{0}\right]
$$

So, the optimum control may not be an instantaneous function of $y(t)$.

The solution of this problem is known as the 'separation theorem'. This theorem says that for linear systems subjected to additive white Gaussian noise inputs with quadratic index of performance, the optimal stochastic controller is determined by combining an optimal estimator with a deterministic (noise-free) optimum controller. Figure 3.8 shows a block-diagram of the linear stochastic optimal regulator problem and illustrates the separation theorem.

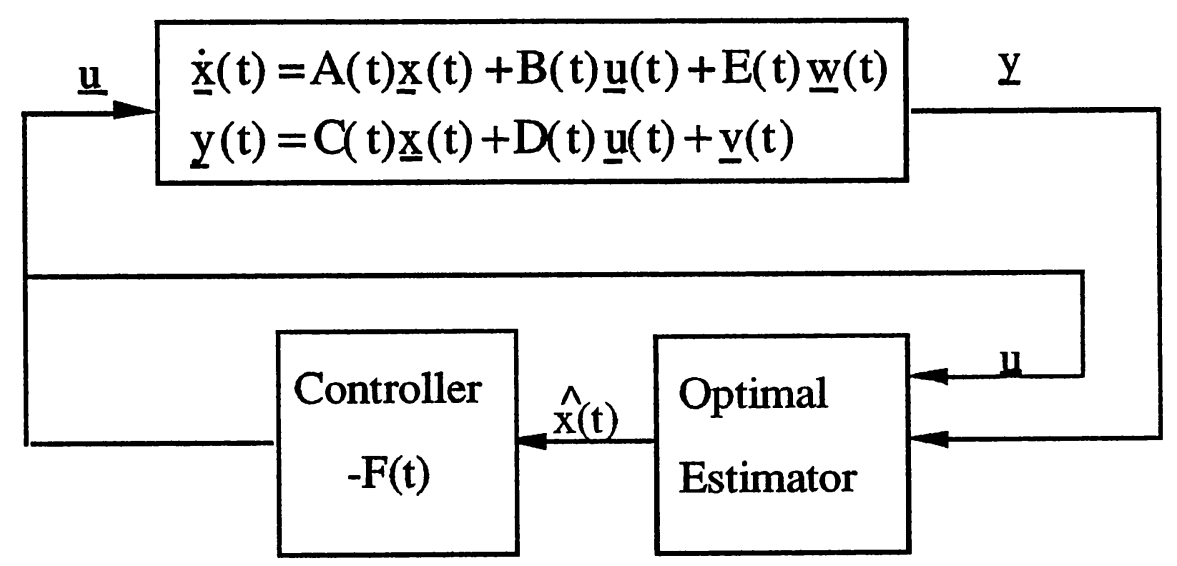

Figure 3.8. Optimal controller for linear stochastic systems 
The linear stochastic optimal controller is determined as follows:

1) Compute the optimal noise-free control law $\underline{u}(t)=-F(t) \underline{x}(t)$.

The calculation of $F(t)$ is independent of $C(t), D(t)$ and input noises.

2) Compute the optimal state estimator of $\underline{x}(t)$ using $\underline{u}(\tau)$ and $\underline{y}(\tau)$ for $\tau$ in the range $\left[t_{0}, t\right]$.

3) Use the control law

$\underline{u}^{*}(t)=-F(t) \underline{\hat{x}}(t)$, where $\underline{\hat{x}}(t)$ is obtained in step 2 .

The calculation of the observer gain matrix $K(t)$ and the controller gain $F(t)$ can be done independently. This separability of the problem is due to the fact that the system is linear and the stochastic processes are white noises. Because of their unpredictability, the white noise processes are not considered in the design of the optimal controller.

The separation theorem cannot be applied for any general nonlinear optimal stochastic problem. We cannot determine the control and estimation strategies independently for the most general stochastic problems. Figure 3.8 diagrams the linear stochastic optimal controller.

\subsection{Optimal Tracking Problems}

\subsubsection{Noise Free Tracking Problem}

The preceding section formulates and solves the problem of returning a system to zero state in some optimal way (optimal regulator problem). This section considers tracking problems in which 
the output of a system (plant) must follow a desired trajectory optimal-wise.

Assume that we are given the following plant.

$$
\begin{aligned}
& \underline{\dot{x}}(t)=A \underline{x}(t)+B \underline{u}(t) \\
& \underline{y}(t)=C \underline{x}(t)
\end{aligned}
$$

where
x -.-- n- dimensional state vector
u ---- r-- dimensional input vector
y ---- m-dimensional output vector
A ---- $n$ by $n$ dimensional constant matrix
B ---- $\mathrm{n}$ by $\mathrm{r}$ dimensional constant matrix
$\mathrm{C}$-.-- $\mathrm{n}$ by $\mathrm{m}$ dimensional constant matrix

We wish to find an input $\underline{u}^{*}(t)$ such that $y(t)$ tracks the desired output $\underline{\tilde{y}}$ which is the output of the reference mode

$$
\begin{gathered}
\underline{\dot{z}}(t)=A_{z} \underline{z}(t) \\
\underline{\tilde{y}}=C_{z} \underline{z}(t)
\end{gathered}
$$

where

$\underline{\mathbf{z}}$-.-- p-dimensional reference state vector

$\underline{\mathrm{y}}$-.-- m-dimensional output vector(desired trajectory)

$A_{z}$--- $p$ by $p$ dimensional constant matrix

$\mathrm{C}_{\mathrm{z}}$--- $\mathrm{p}$ by $\mathrm{m}$ dimensional constant matrix

and

$\left[A_{z}, C_{z}\right]$ is completely observable. 
The optimal tracking problem is defined as:

For the plant model (3.33), find a control input, $\underline{\mathbf{u}}^{*}(\mathrm{t})$ which minimizes the cost functional

$$
J=\int_{t_{0}}^{T}\left\{\underline{u}^{\prime} R \underline{u}+(\underline{x}-\underline{\tilde{x}})^{\prime} Q \underline{(\underline{x}-\underline{\tilde{x}})}\right\} d t
$$

where

$\underline{\tilde{x}}=\mathrm{L} \underline{\tilde{y}}$

Q--- an $\mathrm{n}$ by $\mathrm{n}$ constant non-negative definite symmetric matrix $\mathrm{R}---$ an $\mathbf{r}$ by $\mathbf{r}$ constant positive definite symmetric matrix Here, we assume that the reference state $\underline{z}$ is directly measurable. Let us define the new state $\underline{x}_{T}$ and matrices $A_{T}$ and $B_{T}$ as:

$$
\underline{x}_{T}(t)=\left[\begin{array}{l}
\underline{x}(t) \\
\underline{z}(t)
\end{array}\right], A_{T}=\left[\begin{array}{cc}
A & 0_{n \times p} \\
0_{p \times n} & A_{z}
\end{array}\right], B_{T}=\left[\begin{array}{c}
B \\
0_{p \times r}
\end{array}\right]
$$

By introducing the new state $\underline{x}_{T}$ and new matrices $A_{T}$ and $B_{T}$, we are trying to convert the servo problem to a regulator problem with the following augmented state equation.

$$
\underline{\dot{x}}_{T}(t)=A_{T} \underline{x}(t)+B_{T} \underline{u}(t)
$$

Note that the the above system is not completely controllable, it has $p$ uncontrollable states. The performance index $\mathrm{J}$ is modified as follows. First, by using equations (4.46) and (3.44), we write the difference between $\underline{x}$ and $\underline{\tilde{x}}$ in term of $\underline{x} T$,

$$
\underline{x}(t)-\underline{\tilde{x}}(t)=\underline{x}(t)-L C_{z} \underline{z}(t)=\left[\begin{array}{ll}
I & -L C_{z}
\end{array}\right] \underline{x}_{T} .
$$




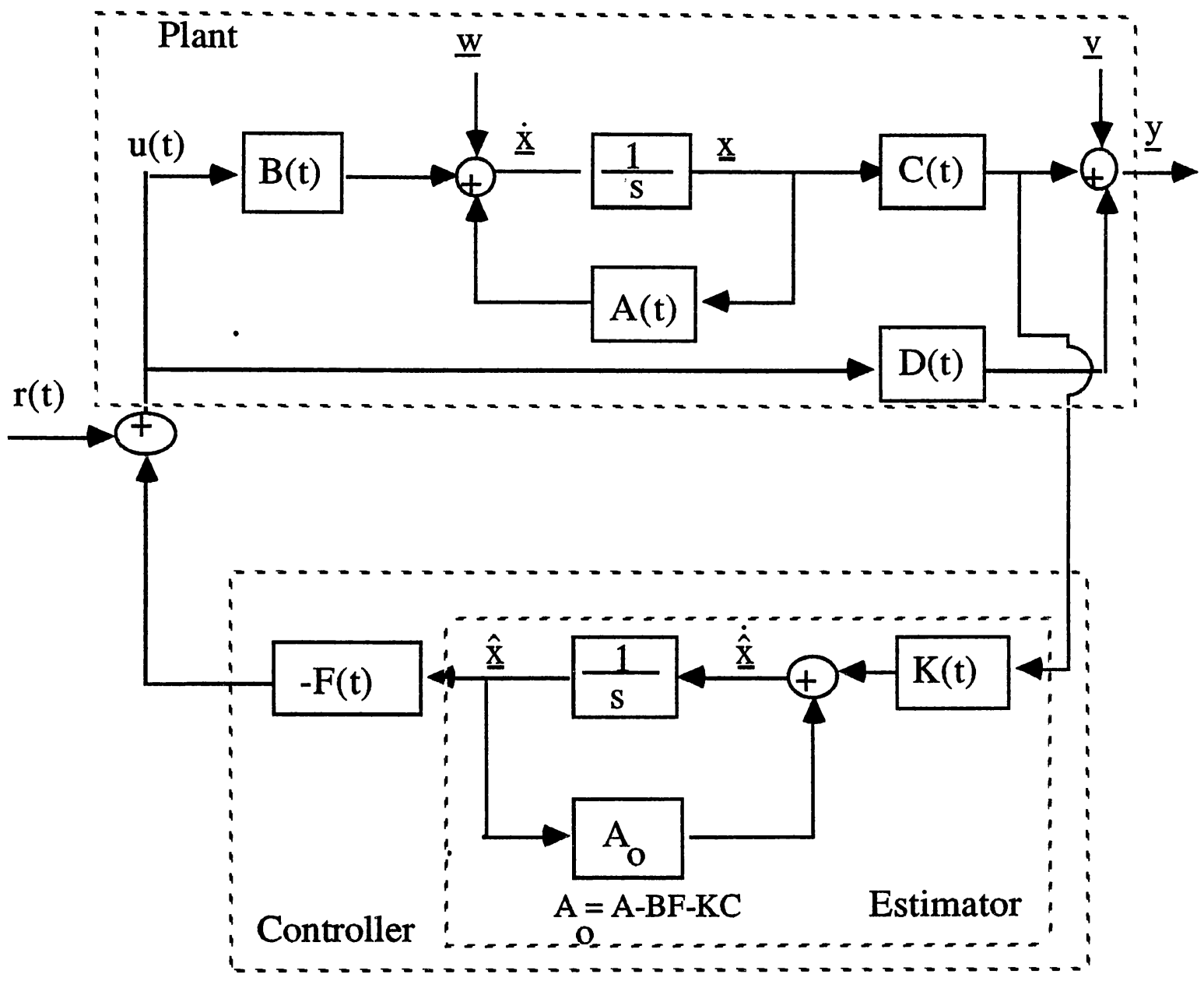

Figure 3.9. Block-Diagram Of Linear Optimal Stochastic Regulator 
Then, the second term in index $J$, equation (3.35) is written in term of $\underline{\mathrm{x}} \mathrm{T}$ as:

$$
\begin{aligned}
& (\underline{x}(t)-\underline{\tilde{x}}(t))^{\prime} Q \underline{x}(t)-\underline{\tilde{x}}(t)=\underline{x}_{T}^{\prime}(t)\left[\begin{array}{c}
I \\
-C^{\prime} L^{\prime}
\end{array}\right] Q\left[\begin{array}{ll}
I & -L C] \underline{x}_{T}(t)
\end{array}\right. \\
& =\underline{x}_{T}^{\prime}(t)\left[\begin{array}{cc}
Q^{\prime} & -Q L C \\
-C^{\prime} L^{\prime} Q & C^{\prime} L^{\prime} Q L C
\end{array}\right] \underline{x}_{T}(t) \text {. }
\end{aligned}
$$

Hence, the index $\mathrm{J}$ in terms of $\underline{x}_{T}$ becomes

$$
J=\int_{t_{0}}^{T}\left\{\underline{u}^{\prime}(t) R \underline{u}(t)+\underline{x}_{T}^{\prime}(t) Q_{T} \underline{x}_{T}(t)\right\} d t
$$

where

$$
Q_{T}=\left[\begin{array}{cc}
Q^{\prime} & -Q L C \\
-C^{\prime} L^{\prime} Q & C^{\prime} L^{\prime} Q L C
\end{array}\right] \text {. }
$$

Now, we can apply the noise-free regulator problem, section 3.5, which yields the optimal control input, $\underline{\mathbf{u}} *(t)$ as:

$$
\underline{u}^{*}(t)=-R^{-1} B_{T}^{\prime} P_{T} \underline{x}_{T}
$$

where $\mathrm{P}_{\mathrm{T}}$ is the solution of the positive definite matrix Riccati equation

$$
P_{T} A_{T}+A_{T}^{\prime} P_{T}-P_{T} B_{T} R^{-1} B_{T}^{\prime} P_{T}+Q_{T}+\dot{P}_{T}=0(n+p) \times(n+p)
$$

with $P_{T}(T)=0$. The minimum performance index is

$$
\mathrm{J}^{* \cdot}=\underline{x}_{\mathrm{T}}^{\prime}\left(\mathrm{t}_{0}\right) \mathrm{P}_{\mathrm{T}}\left(\mathrm{t}_{0}\right) \underline{\mathrm{x}}\left(\mathrm{t}_{\mathbf{0}}\right)
$$


In order to see the effect of the plant parameters on the optimal controller gain matrix, we may continue as follows. Let us partition

$$
\mathrm{P}_{\mathrm{T}}=\left[\begin{array}{cc}
\mathrm{P}_{\mathrm{n} \times \mathrm{n}} & \mathrm{P} 1_{\mathrm{n} \times \mathrm{p}} \\
\mathrm{P} 1^{\prime} & \mathrm{P} 2_{\mathrm{p} \times \mathrm{p}}
\end{array}\right]
$$

The controller gain $\mathrm{F}$ becomes

$$
\begin{aligned}
F= & R^{-1} B_{T}^{\prime} P_{T}=R^{-1}\left[\begin{array}{l}
B \\
0
\end{array}\right]\left[\begin{array}{ll}
B & 0
\end{array}\right]\left[\begin{array}{cc}
P & P 1 \\
P 1 & P 2
\end{array}\right]= \\
& {\left[\begin{array}{ll}
R^{-1} B P & R^{-1} B P 1
\end{array}\right]=\left[\begin{array}{ll}
f_{x} & f_{z}
\end{array}\right], }
\end{aligned}
$$

where

$$
f_{x}=R^{-1} B P \text { and } f_{z}=R^{-1} B P 1
$$

Equation (3.51) is rewritten as

$$
\underline{u}^{*}(t)=-f_{x} \underline{x}(t)-f_{z} \underline{z}(t) .
$$

The Riccati equation (3.42) becomes

$$
\begin{aligned}
& {\left[\begin{array}{cc}
\mathrm{P} & \mathrm{P} 1 \\
\mathrm{P} 1 & \mathrm{P} 2
\end{array}\right]\left[\begin{array}{cc}
\mathrm{A} & 0 \\
0 & \mathrm{~A}_{\mathrm{z}}^{\prime}
\end{array}\right]+\left[\begin{array}{cc}
\mathrm{A}^{\prime} & 0 \\
0 & \mathrm{~A}_{\mathrm{z}}^{\prime}
\end{array}\right]\left[\begin{array}{cc}
\mathrm{P} & \mathrm{P} 1 \\
\mathrm{P} 1^{\prime} & \mathrm{P} 2
\end{array}\right]} \\
& -\left[\begin{array}{cc}
\mathrm{P} & \mathrm{P} 1 \\
\mathrm{P} 1 & \mathrm{P} 2
\end{array}\right]\left[\begin{array}{l}
\mathrm{B} \\
0
\end{array}\right] \mathrm{R}^{-1}\left[\begin{array}{ll}
\mathrm{B}^{\prime} & 0
\end{array}\right]\left[\begin{array}{cc}
\mathrm{P} & \mathrm{P} 1 \\
\mathrm{P} 1 & \mathrm{P} 2
\end{array}\right] \\
& +\left[\begin{array}{cc}
\mathrm{Q} & -\mathrm{QLC} \\
-\mathrm{C}^{\prime} \mathrm{L}^{\prime} \mathrm{Q} & \mathrm{C}^{\prime} \mathrm{L}^{\prime} \mathrm{QLC}
\end{array}\right]+\left[\begin{array}{cc}
\dot{\mathrm{P}} & \dot{\mathrm{P}} 1 \\
\dot{\mathrm{P}} 2 & \dot{\mathrm{P}} 2
\end{array}\right]=0 \text {. }
\end{aligned}
$$


The following equations solve for $\mathrm{p}, \mathrm{p}_{1}$ and $\mathrm{p}_{2}$.

$$
\begin{aligned}
& \mathrm{PA}+\mathrm{A}^{\prime} \mathrm{P}-\mathrm{PBR}^{-1} \mathrm{~B}^{\prime} \mathrm{P}+\mathrm{Q}+\dot{\mathrm{P}}=0_{\mathrm{n} \times \mathrm{n}} \\
& \mathrm{P} 1 \mathrm{~A}_{\mathrm{z}}+\mathrm{AP} 1-\mathrm{PBR}^{-1} \mathrm{~B}^{\prime} \mathrm{P} 1-\mathrm{Q} \mathrm{C}+\dot{\mathrm{P}} 1=0_{\mathrm{n} \times \mathrm{p}} \\
& \dot{\mathrm{P}} 2 \mathrm{~A}_{\mathrm{z}}+\mathrm{A}_{\mathrm{z}} \mathrm{P} 2-\mathrm{P} 2 \mathrm{BR}^{-1} \mathrm{~B}^{\prime} \mathrm{P} 2+\mathrm{C}^{\prime} \mathrm{L}^{\prime} \mathrm{QLC}+\dot{\mathrm{P}} 2=0_{\mathrm{p} \times \mathrm{p}}
\end{aligned}
$$

with the boundary conditions

$$
P(T)=0_{n \times n}, p 1(T)=0_{n \times p}, P 2(T)=0_{p \times p}
$$

In order to determine $f_{x}$, we need to solve equation (3.45a) for P. This solution is dependent only on plant parameters A and B. Figure 3.10 shows the regulator control of the augmented system $\underline{x} T$.

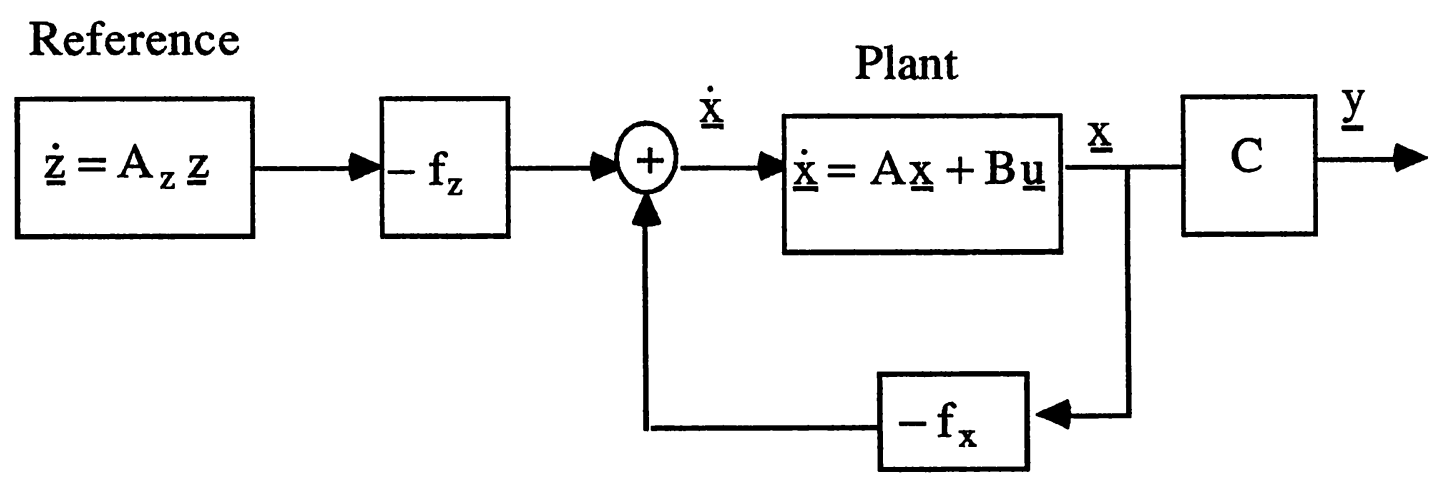

Figure 3.10. Block Diagram of the Regulator Control of the Augmented System $\underline{\mathrm{x} T}$ (Tracking Problem) 
The above results are based on the assumption that the reference state vector $\underline{z}$ is completely measurable. For the case in which $\underline{z}$ is not measurable, the technique in section 3.6 has to be applied to construct a state estimator with $\underline{\tilde{y}}(t)$ as an input and an estimator $\underline{\tilde{x}}$ as an output (see Figure 3.11). The next section considers these kinds of tracking problems where the states are not directly measurable.

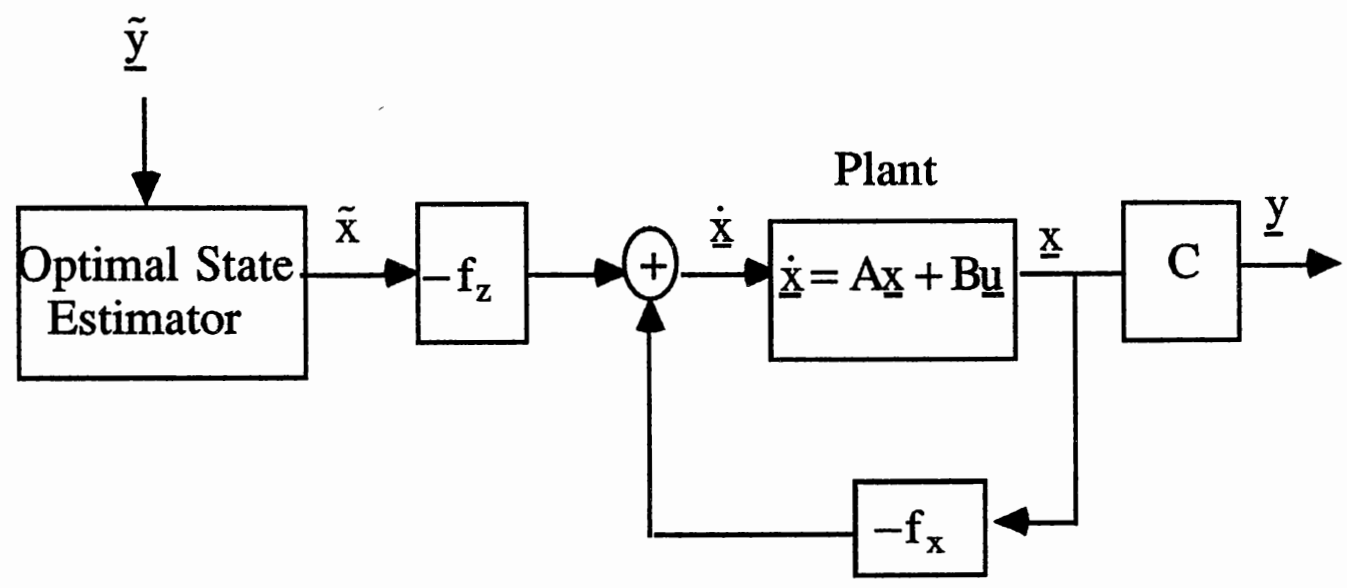

Figure 3.11. Block-Diagram of the Optimal Tracking Problem 


\subsubsection{Linear Optimal Stochastic}

\section{Tracking Problem}

Section 3.8.1 considers a tracking problem where the state of the plant and the state of the reference model are assumed to be directly measurable. This section considers a tracking problem in which certain noisy linear combinations of the state components of both reference and plant can be measured.

Let us assume that the following equations define the reference model

$$
\begin{aligned}
& \underline{\dot{z}}(t)=A_{z} \underline{z}(t)+E_{z} \underline{w}_{z}(t) \\
& \underline{\tilde{y}}(t)=C_{z} \underline{z}(t)
\end{aligned}
$$

where

$\underline{z}\left(t_{0}\right)$ is a stochastic process with mean $\underline{z}_{0}$ and intensity $Q_{z_{0}}$.

$\underline{w}_{z}(t)$ is white noise with mean zero and intensity $W_{z}$. $\underline{v}_{z}(t)$ is a white noise process with mean 0 and intensity $V$.

The plant is modeled as

$$
\begin{aligned}
& \underline{\dot{x}}(t)=A \underline{x}(t)+B \underline{u}(t)+E_{x} \underline{w}_{x}(t) \\
& \underline{y}(t)=C \underline{x}(t)+\underline{v}_{y}(t)
\end{aligned}
$$

where

$\underline{\mathrm{x}}\left(\mathrm{t}_{0}\right)$ is a stochastic variable with mean $\underline{\mathrm{x}}_{0}$ and intensity $\mathrm{Q}_{\mathrm{x}_{0}}$. $\underline{w}_{x}(t)$ and $\underline{v}_{y}(t)$ are white with intensities $w_{x}$ and $v_{y}$ respectively. 
For the system (3.47), we want to find the input $\underline{u}^{*}(t)$ as a function of $\underline{y}(t)$ and $\underline{\tilde{y}}(t), t_{0} \leq t \leq T$, so that the index (3.48) is minimized with the assumption that all white noises and initial conditions are Gaussian and uncorrelated.

$$
J=E\left\{\int_{t_{0}}^{T}\left\{\underline{u}^{\prime}(t) R \underline{u}(t)+\underline{e}^{\prime}(t) Q \underline{e}(t)\right\} d t\right\}
$$

where

$$
\underline{e}(t)=\underline{y}(t)-\underline{\tilde{y}}(t)
$$

The index $J$ says that the controlled output $y$ has to be as close as possible to $\underline{\tilde{y}}$ while not letting the input $u(t)$ exceeds its maximum level.

In order to convert this tracking problem to a stochastic regulator problem, the following augmented state and matrices are defined.

$$
\underline{x}_{T}(t)=\left[\begin{array}{l}
\underline{x} \\
\underline{z}
\end{array}\right], A_{T}=\left[\begin{array}{cc}
A & 0_{n \times p} \\
0_{p \times n} & A_{z}
\end{array}\right], B_{T}=\left[\begin{array}{c}
B \\
0_{p \times r}
\end{array}\right], E_{T}=\left[\begin{array}{c}
E_{x} \\
E_{z}
\end{array}\right],
$$

and

$$
\mathrm{C}_{T}=\left[\begin{array}{cc}
\mathrm{C} & 0_{\mathrm{m} \times \mathrm{p}} \\
0_{\mathrm{m} \times \mathrm{n}} & \mathrm{C}_{\mathrm{z}}
\end{array}\right], \mathrm{v}_{\mathrm{T}}(\mathrm{t})=\left[\begin{array}{c}
\mathrm{v}_{\mathrm{x}}(\mathrm{t}) \\
\mathrm{v}_{\mathrm{z}}(\mathrm{t})
\end{array}\right], \mathrm{y}_{T}=\left[\begin{array}{c}
\underline{y}(\mathrm{t}) \\
\tilde{\tilde{y}}(\mathrm{t})
\end{array}\right], \mathrm{w}_{T}(\mathrm{t})=\left[\begin{array}{c}
\mathrm{w}_{\mathrm{x}}(\mathrm{t}) \\
\mathrm{w}_{\mathrm{z}}(\mathrm{t})
\end{array}\right] \text {. }
$$


Now, we can write the following augmented equations

$$
\begin{aligned}
& \underline{\dot{x}}_{T}(t)=A_{T} \underline{x}_{T}(t)+B_{T} \underline{u}(t)+E_{T} \underline{w}_{T}(t) \\
& \underline{y}_{T}(t)=C_{T} \underline{x}_{T}(t)+\underline{v}_{T}(t)
\end{aligned}
$$

The index $\mathbf{J}$ can be rewritten in terms of $\underline{x}_{T}(t)$ as follows.

$$
\underline{e}(t)=C \underline{x}(t)-C_{z} \underline{z}(t)=\left[\begin{array}{ll}
C & -C_{z}
\end{array}\right] \underline{x}=C_{e} \underline{x}_{T}
$$

By using (3.50) in (3.48), the index $\mathrm{J}$ becomes

$$
J=E\left\{\int_{t_{0}}^{T}\left\{\underline{u}^{\prime}(t) R \underline{u}(t)+\underline{x}_{T}^{\prime}(t) Q_{T} \underline{x}_{T}(t)\right\} d t\right\}
$$

where

$$
\mathrm{Q}_{\mathrm{T}}=\mathrm{C}_{\mathrm{e}}^{\prime} \mathrm{QC}_{\mathrm{e}} \text { and } \mathrm{C}_{\mathrm{e}}=\left[\begin{array}{ll}
\mathrm{C} & -\mathrm{C}_{\mathrm{z}}
\end{array}\right]
$$

So, the tracking problem is stated as: for the system (3.49) find an optimal control input which minimizes the index $J$ given in Equation (3.51). This problem is in the form of the stochastic regulator problem that has been solved in section 3.7

Figure 3.12 shows the structure of the optimal stochastic linear tracking problem. We need to construct two separate state estimators, one for plant state $\underline{\mathrm{x}}$ and one for the state of the reference model, $\underline{\mathrm{z}}$. As before, the optimal controller has the form

$$
\underline{u}^{*}(t)=-F \underline{\hat{x}}(t)=-\left[\begin{array}{ll}
f_{x} & f_{z}
\end{array}\right]\left[\begin{array}{l}
\hat{\hat{x}} \\
\hat{z}
\end{array}\right]=-f_{x} \underline{\hat{x}}-f_{z} \underline{\hat{z}} .
$$


Again, it is seen that the feedback link of the optimal controller is completely independent of the reference model parameters.

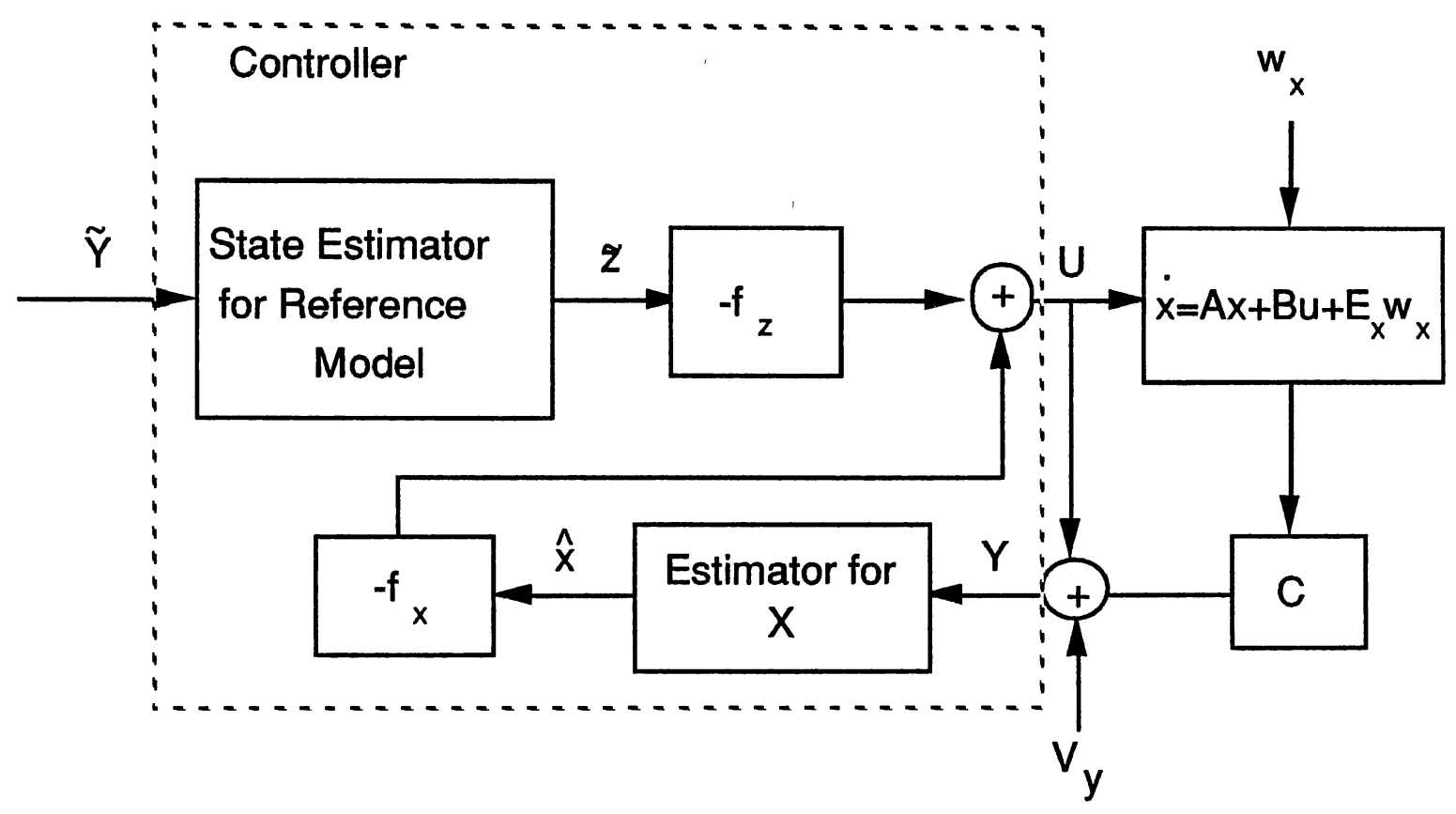

Figure 3.12. A Linear Optimal Stochastic Tracking Problem With

\subsection{Summary}

In this chapter we presented some mathematical preliminaries which are fundamental to the work of subsequent chapters. After presenting some notation, definitions and some results concerning linear dynamic systems and linear optimal control models, a general stochastic optimal control problem was stated. The linear optimal 
regulator problems and their solutions have been discussed in section 3.4 and it has been shown that for linear time-invariant stabilizable and detectable systems, the infinite time regulator problem possesses a constant linear control law leading to an asymptotically stable closed-loop system.

When the states of a system are not measurable, then the separation theorem discussed in section 3.6 says that the optimal control law is to use an optimal full state feedback design, but to substitute state estimates for the actual states. Section 3.6 considered state estimation including the case in which we have additive Gaussian noise on the measurements. The control law synthesis, which represents the solution of a linear stochastic optimal control problem, using full state feedback design and state estimation, was discussed in section 3.7.

Optimal tracking problems, a wider class of regulator problems, have been discussed in section 3.8. First we found control laws which require that both the state of the plant and the reference model be available. In this case, the feedback link of the controller is completely independent of the reference model parameters. Then, we considered a linear stochastic tracking problem, in which a linear combination of the states can be measured. In addition, these measurements are contaminated by additive Gaussian white noise. It has been shown that in order to synthesize the optimal controller, we need to construct two separate state estimators, one for the plant state and one for the reference state. 


\section{CHAPTER IV}

\section{OPTIMAL CONTROL MODEL (OCM)}

\subsection{Introduction \& Background}

As mentioned in chapter II, modeling the input-output characteristic of the human operator in a man-machine system has been extensively investigated in the literature, starting with Tustin [42] and Bates et al. [17] in 1947, followed by Elkind [14] in 1956, and continuing with the investigations of McRuer et al. into mathematical descriptions of human operator in 1957 [15] and human pilot dynamics in 1965 [50,53]. To that point of time, only classical control theory had been applied to manually-controlled systems, i.e. CrossOver modeling [41].

The Cross-Over models, discussed in chapter II, can only be applied to manual control systems which are restricted to be singleinput, single-output, linear and time-invariant. Therefore, they are of no use for multivariable time varying systems.

The earliest application of optimal control theory to modeling a human operator in man-machine systems was made by Thomas in 1962, Obermayer et al. in 1965 and Elkind et al. in 1968 [40,50]. They attempted to combine the human response theory with the analytical techniques of modern control theory in order to predict human-system performance. They had little success because they 
didn't account for limitations which arise due to controller remnant [40,49]. In 1970, Kleinman et al. [49 ] applied optimal estimation and stochastic control theory in order to synthesize the human operator as an optimal stochastic controller. This controller consists of a Kalman filter (optimal state estimator) and a least mean square state predictor, cascaded with the optimal noise-free controller gain factor. This model, known as an optimal control model (OCM) became very powerful and attractive for predicting human performance behavior in simple tasks as well as complex tasks. Section 4.2 discusses this model in detail.

In general, the OCM is a time-domain methodology which combines the human response theory $[27,46]$ and the analytical techniques of the optimal control theory to describe a complex manual control system. The human response theory provides a complete representation of the human operator's psychophysical limitations, and the optimal control theory provides a tool to predict the closed-loop human-system performance.

The OCM is mainly based on the assumption that a well-trained, well-motivated human operator will behave optimally subject to its inherent psychophysical constraints, (whereas the Cross-over model is based on the stability considerations of the closed-loop system). This assumption, which complies with the findings of human behavior in the psychological literature, implies that the human operator, after a period of training, will be acquainted with his own and the system's dynamics (characteristics) including the knowledge of human response capabilities, the statistical nature of the unwanted external noises acting on the controlled system and the task objectives. The 
OCM represents internally the human's inherent constraints and includes elements that optimally compensate for these constraints.

The following section considers the Kleinman-Baron-Levison (KBL) model of the human operator [49] and illustrates the general description of the OCM in more detail.

\subsection{Kleinman-Baron-Levison's Model}

\subsubsection{General Description \& Assumptions}

Figure 4.1 diagrams the general structure of the OCM. The top path represents the controlled system dynamics, which are represented by a linear state vector, and display variables which are linear combinations of the system states and controlled inputs. As indicated in Figure 4.1, the external state disturbance input is considered to be Gaussian white noise, and it can be prefiltered and injected into the control dynamics (additive to the control input). The lower paths in Figure 4.1, represent the human operator with its inherent constraints, represented by the effective time delay $\tau$ and remnant sources, including observation and motor noise. The observation noise is applied to the displayed variables and the motor noise is injected to the controller output. The rest of the human operator components in Figure 4.1, are adaptive. They are adjusted in the sense that a mean square cost functional of states and control efforts is optimized. In other words, the human operator contains elements related to its adaptive behavior and related to inherent limitations which constrain this behavior. 
The optimal control model depicted in Figure 4.1 is based on the following assumptions.

i) The human operator, pilot, is well-trained to behave in a nearoptimal way subject to its own limitations and the system's task requirements.

ii) The controlled system is described by a stochastic linear timeinvariant finite-dimensional dynamic system.

iii) The performance objective is represented by a scalar quadratic performance function of error and control effort.

iv) The unwanted disturbance noises acting on the controlled system, i.e. state disturbance, observation noise and motor noise, are represented as stationary filter processes driven by white noise inputs.

Figure 4.1 also emphasizes that the basic task of human operator is to observe a linear combination of the states corrupted by noise and to generate a control input, in some prescribed way, in order to control a linear dynamical system subjected to some random external noises. Here it is further assumed that the human operator manipulates a single control input $u(t)$ through manipulator devices, e.g., a control stick in pilot modeling. 
White noise

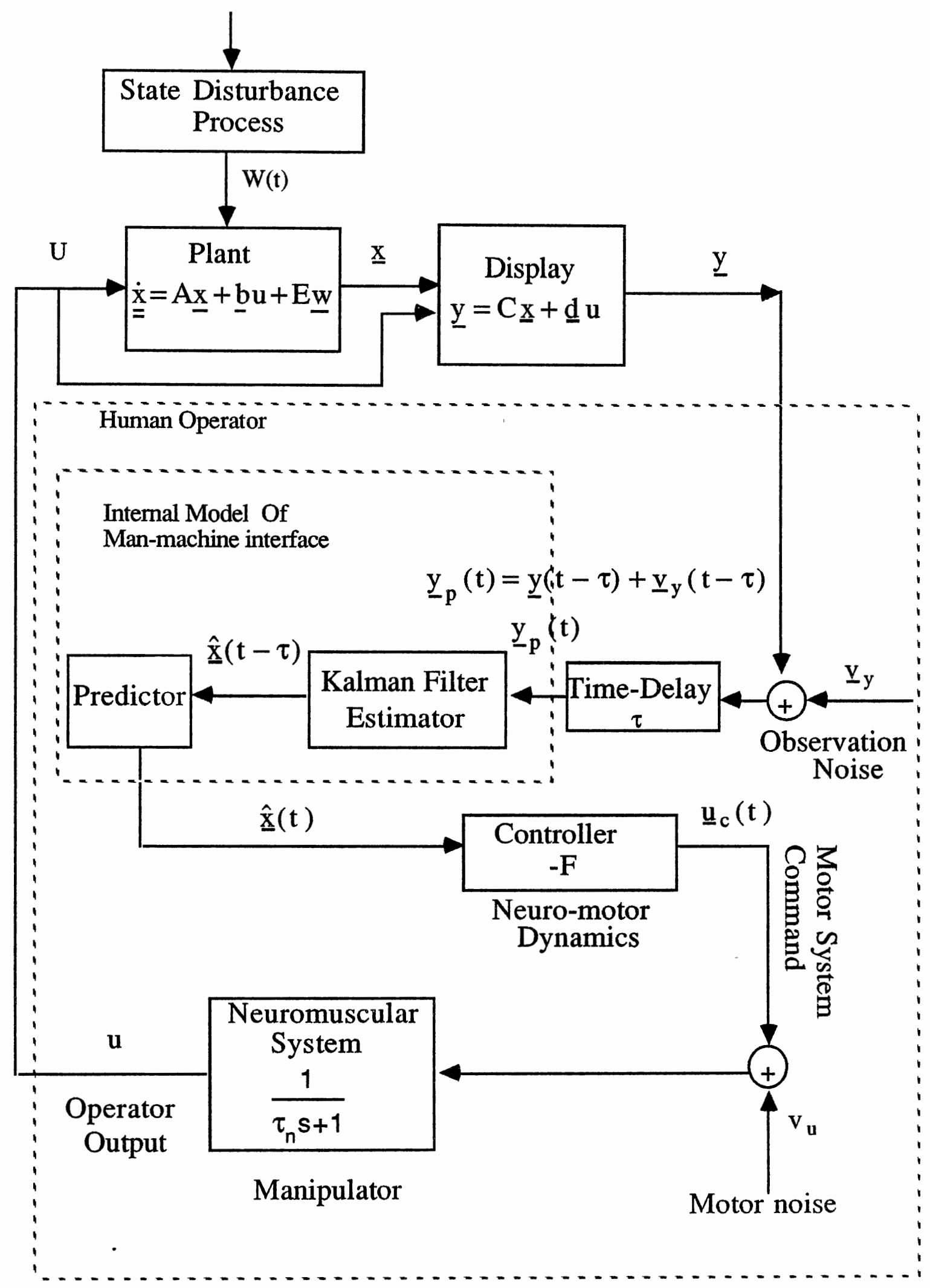

Figure 4.1 OCM Model Structure. 


\subsubsection{Vehicle Dynamics, Task Description and}

\section{Representation Of Human Operator's Limitations}

It is assumed that the vehicle dynamics can be adequately represented by the linear time-invariant equations

$$
\underline{\dot{x}}(t)=A \underline{x}(t)+\underline{b u}(t)+E \underline{w}(t)
$$

where $\underline{x}$ is the vehicle state vector of dimension $n$, the scalar $u$ is the human operator (pilot) control input to the vehicle and $\underline{w}$ is a zero mean white Gaussian state disturbance vector of dimension p (e.g., wind gust) with intensity matrix

$$
\begin{aligned}
& E\left[\underline{w}(t) \underline{w}^{\prime}(\tau)\right]=W \delta(t-\tau), \\
& E\left[w_{i}(t) w_{j}(\tau)\right]=\left\{\begin{array}{ll}
w_{i i} \delta(t-\tau) & i=j \\
0 & i \neq j
\end{array}\right\} .
\end{aligned}
$$

For the case when $\underline{w}(t)$ is colored noise, a linear process driven by white noise can be included in the equation (4.1) by introducing additional states. The visual displayed variables, available system outputs, are linear combinations of state variables and the vehicle's input

$$
\underline{y}(t)=C \underline{x}(t)+\underline{d u}(t)
$$

where $\underline{y}(t)$ is a vector of dimension $m, m \leq n$. Furthermore, since it has been found that when the human operator observes a displayed quantity, $\underline{y}(t)$, he is able to extract the first rate of change of that 
quantity [9,59]. It is assumed that $\underline{\mathrm{y}}(\mathrm{t})$ includes all variables displayed explicitly plus the first derivatives of those variables.

We suppose that the human operator's task is adequately specified in terms of the following cost functional that he attempts to minimize

$$
J(u)=E\left(\underline{x}^{\prime}(t) Q \underline{x}(t)+r u^{2}+g \dot{u}^{2}\right)
$$

where the first term in (4.4) represents mean square error and the rest represents mean square control effort. The human operator attempts to make the mean square error as small as possible while not letting the control efforts exceed some desired maximum level. The cost weighting factors may be objective or subjective. It is objective if the designer specifies them and it will be subjective if the human operator adopts them. There is no guarantee that the controller will adopt subjectively the cost functions which are preassigned objectively [40]. Therefore, it is assumed that the human operator's subjective cost function is of the same form as equation (4.4) and that he may choose different weighting than those assigned by the designer.

Equation (4.4) includes a cost which is dependent on the control rate. This term may represent physiological limitations on the rate at which a human operator can take an action. Rynaski et al. [39] have proven that introducing a control rate term, subjective or objective weight $\mathrm{g}$, in equation (4.4) results in a first order lag in the optimal controller. As a consequence, the quantity $g$ may be used to account for the neuromuscular time constant lag $\tau_{n}$, i.e. the reaction time constant 
of the pilot. We shall see later that the adjusting weight, $g$, is used in order to set the lag $\tau_{\mathrm{n}}$ which is between 0.1 and 0.3 seconds.

Furthermore, it is assumed that the different sources of human randomness can be clearly represented by errors in observing the displayed variables and in executing the desired control movements. Therefore, the observation noise and motor noise represent the lumped effects of controller remnant. Reference [46] considers the controller remnant in detail and shows that over a wide range of single-axis tracking tasks, the equivalent observation noise associated with a displayed quantity can be a Gaussian white noise process, and when a quantity is observed foveally, its associated white observation noise has a intensity (power density) that is proportional to the variance of the observed quantity. The constant of proportionality, the observation noise ratio, does not depend on the system inputs. This quantity is defined as the ratio of the power density of the observation noise to the variance of the associated observed variable.

Thus it is assumed that the observation noises, $\underline{v}_{y_{1}}(t), i=1, \ldots, m$, are linearly independent zero mean, stationary Gaussian white noise processes with intensity $\mathrm{V}_{\mathrm{y}}$.

$$
\begin{aligned}
& E\left[v_{y_{i}}(t) v_{y_{j}}(\tau)\right]=\left\{\begin{array}{ll}
v_{y_{1}} \delta(t-\tau) & i=j \\
0 & i \neq j
\end{array}\right\} \\
& \text { or } \\
& E\left[\underline{v}_{y}(t) \underline{v}_{y}^{\prime}(\tau)\right]=v_{y} \delta(t-\tau)
\end{aligned}
$$

where 


$$
\mathrm{v}_{\mathrm{y}}=\left[\begin{array}{ccccc}
\mathrm{v}_{\mathrm{y}_{1}} & 0 & \cdot & \cdot & 0 \\
0 & \cdot & \cdot & \cdot & \cdot \\
\cdot & 0 & \cdot & \cdot & . \\
\cdot & \cdot & \cdot & \cdot & 0 \\
0 & \cdot & \cdot & 0 & \mathrm{v}_{\mathrm{y}_{\mathrm{m}}}
\end{array}\right], \mathrm{v}_{\mathrm{y}_{\mathrm{i}}}=\Pi \rho_{\mathrm{y}} \mathrm{E}\left\{\mathrm{y}_{\mathrm{p}_{\mathrm{i}}}^{2}\right\}, \mathrm{i}=1,2, \ldots, \mathrm{m}
$$

A typical value of $\rho_{y}$ would be 0.01 , which yields a normalized power level of $-20 \mathrm{db}$. The intensity matrix $V_{y}$ depends on the display's features, such as type and quality and the way the human is viewing the display (foveally or peripherally). Therefore, the human operator perceives a delayed noisy version of the displayed variables. In other words, the pilot experiences $\underline{y}_{p}(t)$ in order to take an action (yielding commanded control input $u_{c}(t)$ ).

$$
\begin{aligned}
\underline{y}_{p}(t) & =\underline{y}(t-\tau)+\underline{v}_{y}(t-\tau) \\
& ={ }_{\underline{x}}(t-\tau)+\underline{d u}(t-\tau)+\underline{v}_{y}(t-\tau) .
\end{aligned}
$$

Because the human operator has no perfect knowledge of the controlled system input, $u(t)$, a motor noise process $v_{u}(t)$, which also represents random errors in executing the desired control movements, is added to the commanded input $u_{c}(t)$. The motor noise $v_{u}(t)$ is a Gaussian white process with variance

$$
\mathrm{v}_{\mathrm{u}}=\Pi \rho_{\mathrm{u}} \mathrm{E}\left(\mathrm{u}_{\mathrm{c}}^{2}\right)
$$

A typical value for $\rho_{u}$ is 0.003 , which gives a normalized motor noise of approximately $-25 \mathrm{db}$. 


\subsubsection{Basic Structure of The OCM}

Figure 4.1 illustrates the general structure of the human operator. It consists of the following two submodels.

\section{i) Perceptual Submodel}

Figure 4.2 shows this submodel in a block-diagram form. This submodel with displayed variables as input and noisy delayed variables as its output accounts for the human operator's own limitations discussed in the preceding section, except for the neuromuscular reaction time delay.

ii) Controller Submodel

The remaining elements of the human operator model (the controller submodel) are illustrated in Figure 4.3. As seen in this Figure, the Kalman filter gives the optimal estimation of the delayed state, $\underline{\hat{x}}(t-\tau)$, from the noisy, delayed output variables, $\underline{y}_{p}(t)$ and then the predictor produces the current estimate of state vector, $\underline{\hat{x}}(t)$ , followed by the optimal controller which assigns a set of control gains to the elements of $\underline{\hat{x}}(t)$, yielding the commanded control signal $u_{c}(t)$. The Kalman filter-predictor followed by controller gains represent the adaptations by which the human operator maximizes his performance and compensates for his own constraints [69]. Finally, the first-order neuro-muscular system filters the commanded input plụs noise and generates the control input $u(t)$. This filtering is 


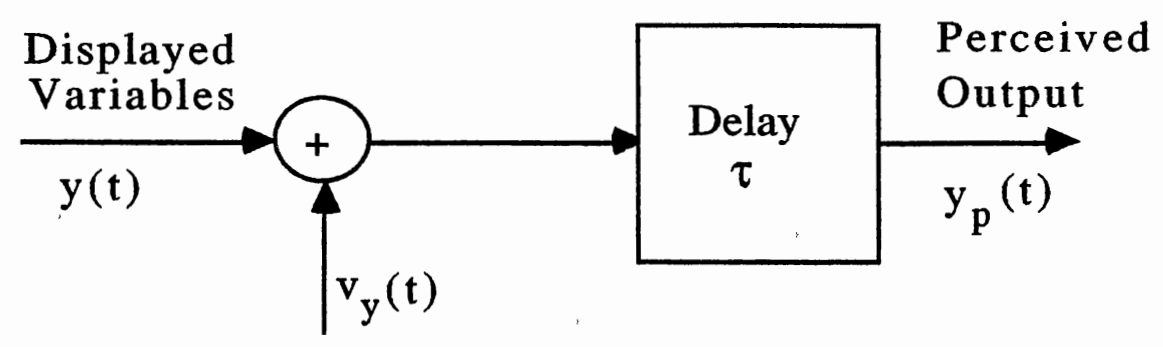

Observation Noise

Figure 4.2. Perceptual Submodel For Human Operator

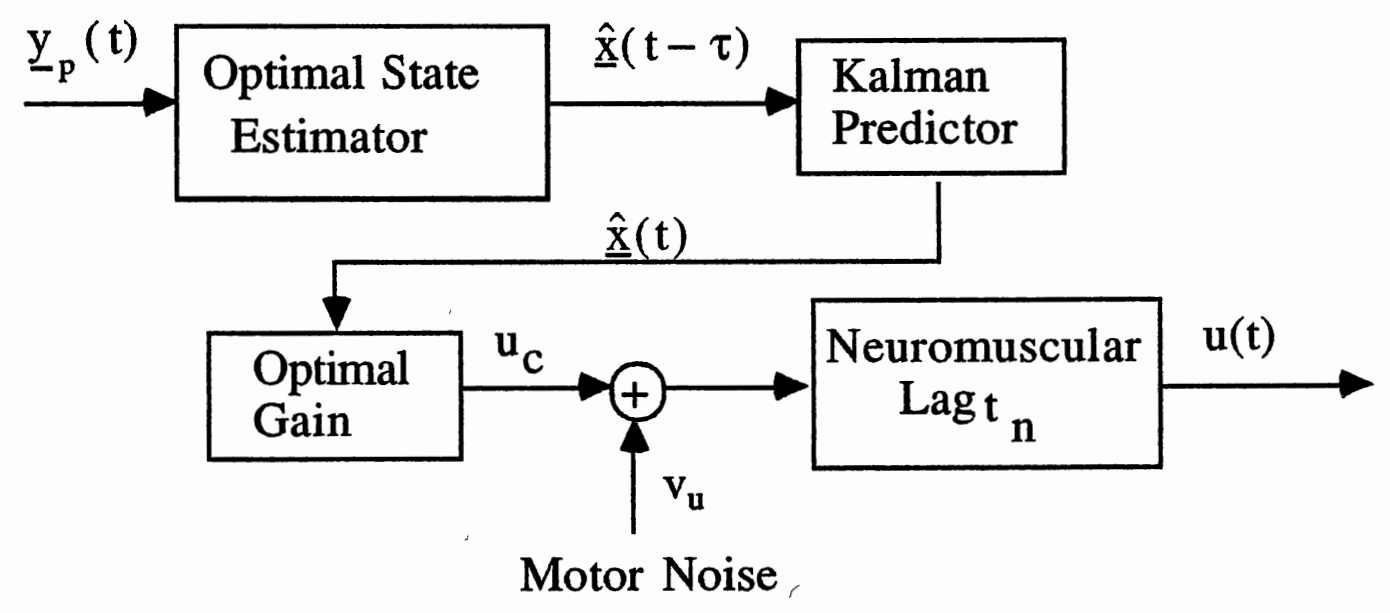

Figure 4.3. The controller Submodel 
The neuro-muscular time lag , $\tau_{\mathrm{n}}$, is typically between 0.09 and 0.12 second for wide bandwidth manual control tasks. In most cases, the desired lag $\tau_{\mathrm{n}}$, may be obtained by correctly choosing of the cost weighting $g$ in equation (4.4).

\subsubsection{Solution Of The Optimal Control Model}

Referring to chapter III, one can see that the human operator's control input characteristics may be determined by the solution of a stochastic linear regulator problem which includes a time delay constraint $\tau$. Reference [70] solves this special kind of stochastic regulator problems Using the techniques in [70], it has been found [49] that the solution of this problem has the essential analytical results which are summarized below.

Since the controlled system is linear and the noise processes acting on the system are Gaussian and white, the separation theorem, discussed in chapter III, can be applied. Let us define the new augmented state

$$
\underline{\chi}(t)=\left[\begin{array}{l}
\underline{x}(t) \\
u(t)
\end{array}\right] .
$$

The following equations are used to solve for the optimal noise-free controller gain matrix which yields the commanded control input $u_{c}(t)$ that minimizes the cost function $J(u)$, equation (4.4).

$$
F=\frac{1}{g} \underline{b}_{1}^{\prime} P=\left[\begin{array}{lllll}
f_{1} & . & f_{n} & f_{n+1}
\end{array}\right]
$$


where the constant matrix $\mathrm{P}$ is the unique positive definite solution of the $(n+1)$-dimensional Riccati equation

$$
A_{1}^{\prime} P+P A_{1}+Q_{0}-\frac{1}{g} \underline{b}_{1} \underline{b}_{1}^{\prime} P=0
$$

with

$$
A_{1}=\left[\begin{array}{cc}
A & \underline{b} \\
0_{1 \times n} & 0
\end{array}\right], \underline{b}_{1}=\left[\begin{array}{c}
0_{n \times 1} \\
1
\end{array}\right], Q_{0}=\left[\begin{array}{cc}
Q & 0_{n \times 1} \\
0_{1 \times n} & r
\end{array}\right]
$$

The human's time constant lag $\tau_{n}$ and optimal controller gain $F^{*}$ are

$$
\begin{aligned}
& \tau_{n}=\frac{1}{f_{n+1}}, \\
& F^{*}=\left[\begin{array}{llll}
f_{1}^{*} & . & f_{n}^{*}
\end{array}\right], f_{i}^{*}=\tau_{n} f_{i}=\frac{f_{i}}{f_{n+1}}, i=1,2, \ldots, n .
\end{aligned}
$$

The optimal control input $u^{*}(t)$ is generated as:

$$
t_{n} \dot{u}^{*}(t)+u^{*}(t)=u_{c}(t)+v_{u}(t)
$$

where the optimal commanded input $u_{c}(t)$ is

$$
u_{c}(t)=-F^{*} \underline{\hat{x}}(t)
$$

The quantity $\underline{\hat{x}}(t)$ is the optimal state estimate of $\underline{x}(t)$ based on the observation output data $\underline{y}_{p}(\tau), \tau \leq t$.

By combining Equations (4.1) and (4.14), $\underline{\chi}(\mathrm{t})$ satisfies

$$
\underline{\dot{\chi}}(\mathrm{t})=\mathrm{A}_{2} \underline{\chi}(\mathrm{t})+\underline{\mathrm{b}}_{2} \mathrm{u}_{\mathrm{c}}(\mathrm{t})+\mathrm{E}_{1} \underline{\mathrm{w}}_{1}(\mathrm{t})
$$


and the state estimate $\underline{\hat{x}}(t)$ is obtained by cascade combination of an optimal state estimator (Kalman filter) and a least mean square predictor. The Kalman filter produces a least mean square delayed estimate, $\underline{\hat{x}}(t-\tau)$, of the delayed state $\underline{x}(t-\tau) b y$

$$
\dot{\dot{\gamma}}(t)=A_{2} \underline{\gamma}(t)+\underline{b}_{2} u_{c}(t-\tau)+K\left[\underline{y}_{p}(t)-C_{2} \gamma(t)\right]
$$

where

$$
\underline{\gamma}(t)=\left[\begin{array}{l}
\hat{\hat{x}}(t-\tau) \\
\hat{u}(t-\tau)
\end{array}\right], A_{2}=\left[\begin{array}{cc}
A & \underline{b} \\
0_{1 \times n} & -\frac{1}{\tau_{n}}
\end{array}\right], \underline{b}_{2}=\left[\begin{array}{c}
0_{n \times 1} \\
\frac{1}{\tau_{n}}
\end{array}\right], C_{2}=\left[\begin{array}{ll}
C & d
\end{array}\right],
$$

and

$$
\underline{w}_{1}(t)=\left[\begin{array}{c}
\underline{w}(t) \\
v_{u}(t)
\end{array}\right], E_{1}=\left[\begin{array}{cc}
E & 0_{n \times 1} \\
0_{1 \times n_{w}} & \frac{1}{\tau_{n}}
\end{array}\right]
$$

The optimal estimator gain matrix $K$ is

$$
\mathrm{K}=\Gamma \mathrm{C}_{2}^{\prime} \mathrm{V}_{\mathrm{y}}^{-1}
$$

where $\Gamma$ is the error intensity matrix and the unique symmetric positive definite solution of the Riccati equation

$$
\Gamma \mathrm{A}_{2}^{\prime}+\mathrm{A}_{2} \Gamma+\mathrm{W}_{1}-\Gamma \mathrm{C}_{2}^{\prime} \mathrm{V}_{\mathrm{y}}^{-1} \mathrm{C}_{2} \Gamma=0_{(\mathrm{n}+1) \times(\mathrm{n}+1)}
$$

with

$$
\mathrm{W}_{1}=\left[\begin{array}{cc}
E W E^{\prime} & 0_{\mathrm{n} \times 1} \\
0_{1 \times \mathrm{n}} & \frac{\mathrm{v}_{\mathrm{u}}}{\tau_{\mathrm{n}}^{2}}
\end{array}\right]
$$


The predictor yields the optimum estimate $\underline{\hat{\chi}}(t)=\left[\begin{array}{l}\underline{\hat{x}}(t) \\ \hat{u}(t)\end{array}\right]$ from the Kalman filter output $\underline{\gamma}(t)$. The following equations define the linear dynamics of the predictor

$$
\begin{aligned}
& \underline{\hat{\chi}}(\mathrm{t})=\underline{\xi}(\mathrm{t})+\mathrm{e}^{\mathrm{A}_{2} \tau}[\underline{\gamma}(\mathrm{t})-\underline{\xi}(\mathrm{t}-\tau)] \\
& \underline{\dot{\xi}}(\mathrm{t})=\mathrm{A}_{2} \underline{\xi}(\mathrm{t})+\underline{\mathrm{b}}_{2} \mathrm{u}_{\mathrm{c}}^{*}(\mathrm{t})
\end{aligned}
$$

and the optimal commanded input is

$$
u_{c}^{*}(t)=-\left[\begin{array}{ll}
F^{*} & 0
\end{array}\right] \underline{\hat{\chi}}(t)
$$

Figure 4.4 shows the structure of the optimal controller in the frequency domain. As seen, the human operator is a linear model and it is possible to find a closed-loop expression for the covariance matrix of $\underline{\chi}(t)$.

$$
\begin{aligned}
X=E\left\{\underline{\chi}(t) \underline{\chi}(t)^{\prime}\right\}= & e^{A_{2} \tau} \Gamma e^{A_{2}^{\prime} \tau}+\int_{0}^{\tau} e^{A_{2} \sigma} W_{1} e^{A_{2}^{\prime} \sigma} d \sigma+ \\
& \int_{0}^{\infty} e^{\bar{A}_{2} \sigma} e^{A_{2} \tau} \Gamma C_{2}^{\prime} V_{y}^{-1} C_{2} \Gamma e^{A_{2}^{\prime} \tau} e^{\bar{A}_{2} \sigma} d \sigma
\end{aligned}
$$

where

$$
\overline{\mathrm{A}}=\mathrm{A}_{2}-\underline{\mathrm{b}}_{2}\left[\begin{array}{ll}
\mathrm{F}^{*} & 0
\end{array}\right]
$$

Since $\underline{\chi}()=.\left[\begin{array}{l}\underline{x}(.) \\ u(.)\end{array}\right]$, the variance of the system quantities are:

$$
E\left[x_{i}^{2}(t)\right]=X_{i i}, \quad \text { For } i=1, \ldots, n
$$




$$
\begin{aligned}
& E\left[y_{i}^{2}(t)\right]=\left\{\left[\begin{array}{ll}
C & d
\end{array}\right] X\left[\begin{array}{l}
C^{\prime} \\
d^{\prime}
\end{array}\right]\right\}_{i i}, \quad \text { For } i=1, \ldots, m \\
& E\left[u^{2}(t)\right]=X_{(n+1),(n+1)} .
\end{aligned}
$$

The effects of human's limitations on the ovearall closed-loop manmachine system are explicitely reflected in the equations (4.24) \& (4.25).

Being linear and time-invariant, the structure of the OCM can be formulated in the frequency domain by a linear transfer function linking $y$ to $u$ in the form

$$
\begin{aligned}
\mathrm{U}(\mathrm{s})=\underline{\mathrm{H}}(\mathrm{s}) \underline{\mathrm{Y}}(\mathrm{s}) & =\left[\begin{array}{lll}
\mathrm{H}_{1}(\mathrm{~s}) & . & \cdot \mathrm{H}_{\mathrm{m}}(\mathrm{s})
\end{array}\right]\left[\begin{array}{c}
\mathrm{Y}_{1}(\mathrm{~s}) \\
\cdot \\
\cdot \\
\mathrm{Y}_{\mathrm{m}}(\mathrm{s})
\end{array}\right] \\
& =\sum_{1}^{\mathrm{m}} \mathrm{H}_{\mathrm{i}}(\mathrm{s}) \mathrm{Y}_{\mathrm{i}}(\mathrm{s}) .
\end{aligned}
$$

From Equations (4.16)-(4.23), we may derive the following expression for $\underline{\mathrm{H}}(\mathrm{s})$.

$$
\underline{H}(s)=-\frac{\left[\begin{array}{ll}
F^{*} & 0
\end{array}\right]}{\tau_{n} s+1}\left\{e^{\left(A_{2}-s I\right) \tau} \cdot(s I-\hat{A})^{-1} \cdot K\right\} \cdot(1+\psi)^{-1}
$$

where $\hat{\mathrm{A}}=\mathrm{A}_{2}-\mathrm{KC}_{2}$ and

$$
\Psi=\left[\begin{array}{ll}
\mathrm{F}^{*} & 0
\end{array}\right] \cdot\left\{\mathrm{e}^{\left(\mathrm{A}_{2}-\mathrm{sI}\right) \tau} \cdot\left[(\mathrm{sI}-\hat{\mathrm{A}})^{-1}-\left(\mathrm{sI}-\mathrm{A}_{2}\right)^{-1}\right]+\left(\mathrm{sI}-\mathrm{A}_{2}\right)^{-1}\right\} \underline{\mathrm{b}}_{2} \cdot
$$

Appendix A presents the solution of the OCM in more detail. 


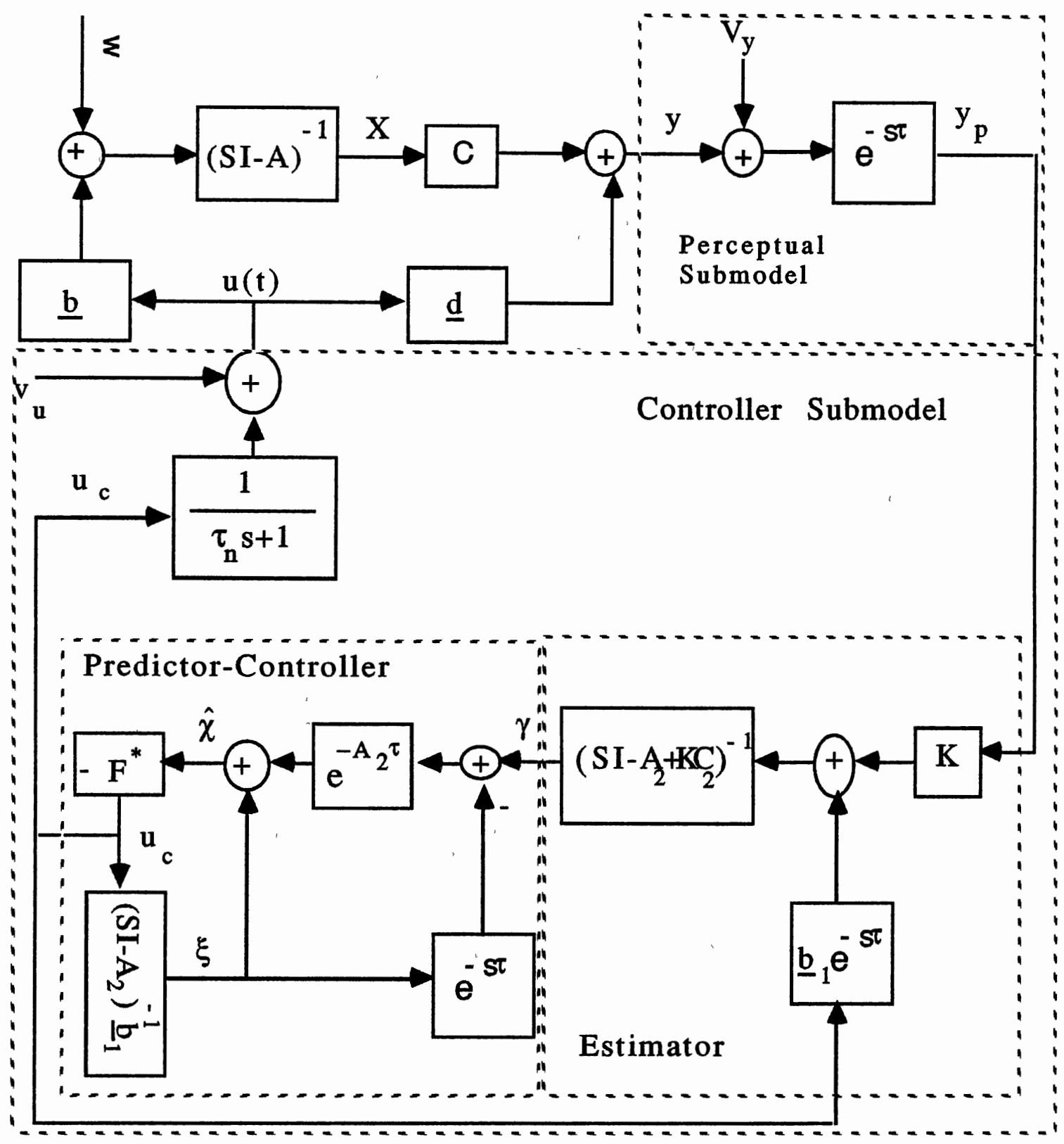

Figure 4.4. Frequency Domain Representation Of The OCM 
In summary, the KBL's OCM (standard OCM) is built up based on the following assumptions.

1. The human operator possesses an internal state space representation of the controlled system and it's environment.

2. The human operator perceives a set of noisy, delayed display variables and their first derivatives.

3. The human operator acts like an optimal full-state estimatorcontroller.

4. The human operator, as an optimal controller, selects a control law which minimizes a quadratic index functional in terms of states and control efforts (including control input and its first derivative).

5. The human operator takes an action in a noisy motor channel.

Under the above assumptions, the OCM consists of three key elements: Kalman filter, minimum mean square predictor, and the optimal controller gain factor. Moreover, for a given controlled system the OCM is completely determined by the quadratic cost weightings, the time delay $\tau$, and the intensities of the observation and motor noise; the effects of human's limitations on the overall closed-loop man-machine system are explicitly reflected in the equations (4.24)(4.26). 


\subsection{Modified OCM (Using Pade Approximant}

For Delay Elements)

As seen in section 4.2, the optimal control model contains a $\tau$ second time delay which reflects the combined effects of delays associated with various inherent constraints of the human operator (e.g., visual \& sensory pathways shown in Figure 4.1). Reference [49] considered this delay in the measurements, see equation (4.6), and solved the OCM problem for this case. Their solution was summarized in the preceding section. In the literature, KBL's OCM is called the standard OCM $[8,71]$ which is discussed in section 4.2.

The standard OCM tries to act as a trained human operator in the sense that it mimics the human tasks of observing, thinking and acting (motor control). As mentioned earlier, it consists of two cascade elements (estimator and predictor in state space form) whose model structure complexities depend on the controlled system structure.

For high order controlled systems, the input/output transfer function for the human operator is of high order, and it has been shown that it is often affected by near pole-zero concellations [72]. Therefore, it is desirable to have a minimally complex OCM model for the human operator.

Because the controlled system is linear, we could insert the $\tau$ second delay element anywhere in the man-machine-loop (see Figure 4.1). Reference [8] considers the case where the delay is imbeded within the state equations (see Figure 4.5) and solves the optimal control problem for this case by employing Pade Approximation technique, which models the delay element $\tau$ as a finite dimensional 
dynamic system. The solution of the modified OCM is presented in this section, after some introduction of Pade Approximant.

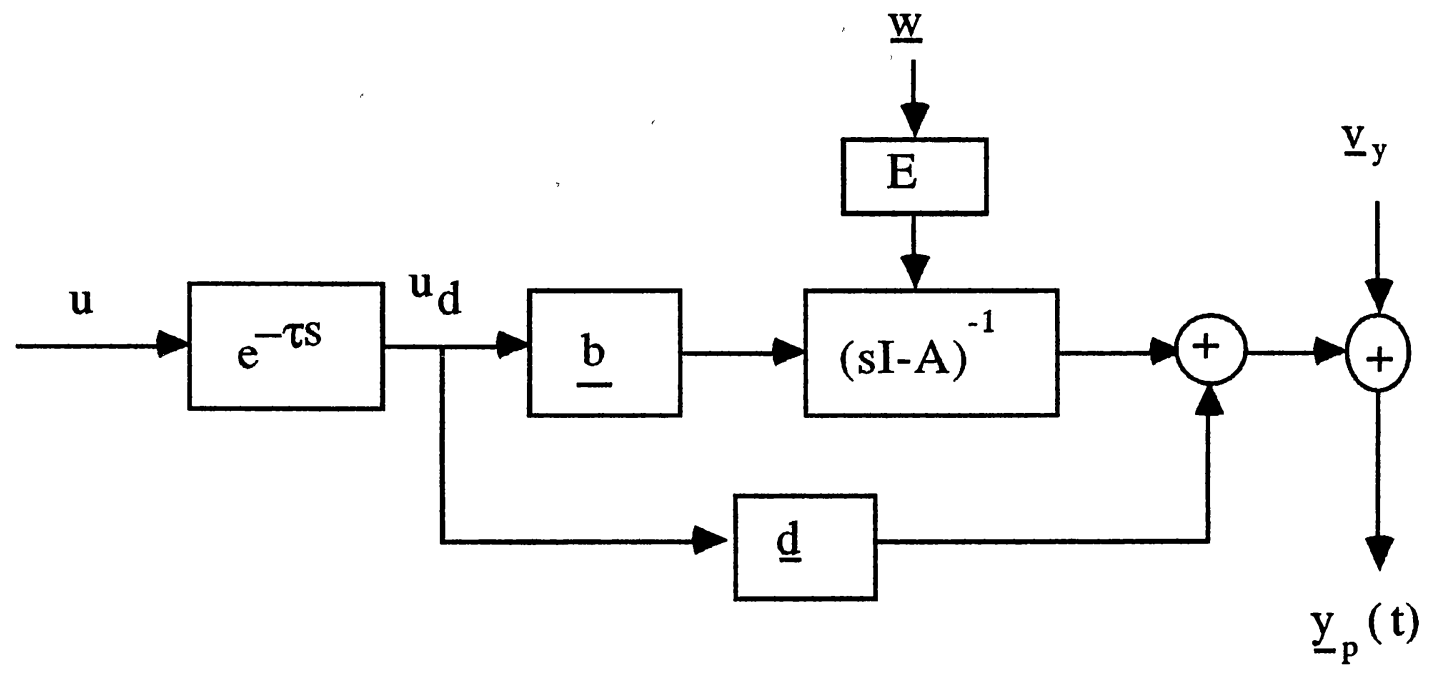

Figure 4.5. Controller System With Perceived Output Variables

\subsubsection{Pade Approximant Technique}

A Pade approximant is nothing but a rational function $\frac{P(.)}{Q(.)}[73]$, where, $\mathrm{P}($.$) is a polynomial of degree \mathrm{m}$ and $\mathrm{Q}($.$) is a polynomial of$ degree $n$. We say that $\frac{P(.)}{Q(.)}$ is a Pade approximant of the function $f($. iff their power series agree up to and including terms of order $x^{m+n}$. Let $f(s)=e^{-\tau s}$ be the function we wish to approximate. The function $\mathrm{f}(\mathrm{s})$ may be defined by the following power series about $s=0$. 


$$
\begin{aligned}
f(s) & =\sum_{i=0}^{i=m+n} \frac{f^{(i)}(0)}{i !} s^{i} \\
& =1+\frac{f^{(1)}(0)}{1 !} s+\frac{f^{(2)}(0)}{2 !} s^{2}+\ldots+\frac{f^{(m+n)}(0)}{(m+n) !} s^{m+n}
\end{aligned}
$$

where

$$
\begin{aligned}
& f^{(j)}(0)=\left.\frac{d^{j} f(x)}{d x^{j}}\right|_{x=0}, \\
& \left.\left(e^{-\tau s}\right)^{(i)}\right|_{s=0}=\left.(-\tau)^{i} e^{-\tau s}\right|_{s=0}=(-\tau)^{i} .
\end{aligned}
$$

Hence, (4.26a) can be rewritten as

$$
\mathrm{e}^{-\tau \mathrm{s}} \cong 1-\tau \mathrm{s}+\frac{\tau^{2}}{2} \mathrm{~s}^{2}-\frac{\tau^{3}}{6} \mathrm{~s}^{3}+\ldots+(-1)^{\mathrm{n}+\mathrm{m}} \frac{\tau^{\mathrm{n}+\mathrm{m}}}{(\mathrm{n}+\mathrm{m}) !} \mathrm{s}^{\mathrm{n}+\mathrm{m}}
$$

Let the Pade approximant be of the form

$$
\frac{p(s)}{Q(s)}=\frac{a_{0}+a_{1} s+\ldots+a_{m} s^{m}}{1+b_{1} s+\ldots+b_{n} s^{n}} \text {. }
$$

Equation (4.27) has to agree with equation (4.28) up to order $s^{m+n}$. To be so, the following set of equations has to be satisfied.

$$
\begin{aligned}
& a_{0}=1 \\
& a_{1}=b_{0}(-\tau)+b_{1}=\sum_{i=0}^{1} b_{i} \frac{(-t)^{1-i}}{(1-i) !} \\
& \vdots \\
& a_{m}=b_{0} \frac{(-t)^{m}}{m !}+b_{1} \frac{(-t)^{m-1}}{(m-1) !}+\ldots+b_{m}=\sum_{1=0}^{m} b_{1} \frac{(-\tau)^{m-i}}{(m-i) !} \\
& \sum_{i=0}^{n} b_{i} \frac{(-\tau)^{m+j-i}}{(m+j-i) !}=0, \quad j=1,2, \ldots, n \\
& \text { where, } b_{0}=1
\end{aligned}
$$


The above $(n+m)$ equations solve uniquely for coefficients of the Pade approximant given in equation (4.28). In order to solve the set of equations (4.29) simultaneously, we may continue as follows.

Let us denote $c_{j}=\frac{(-\tau)^{j}}{j !}$. Equations (4.29) can be rewritten in the following matrix form

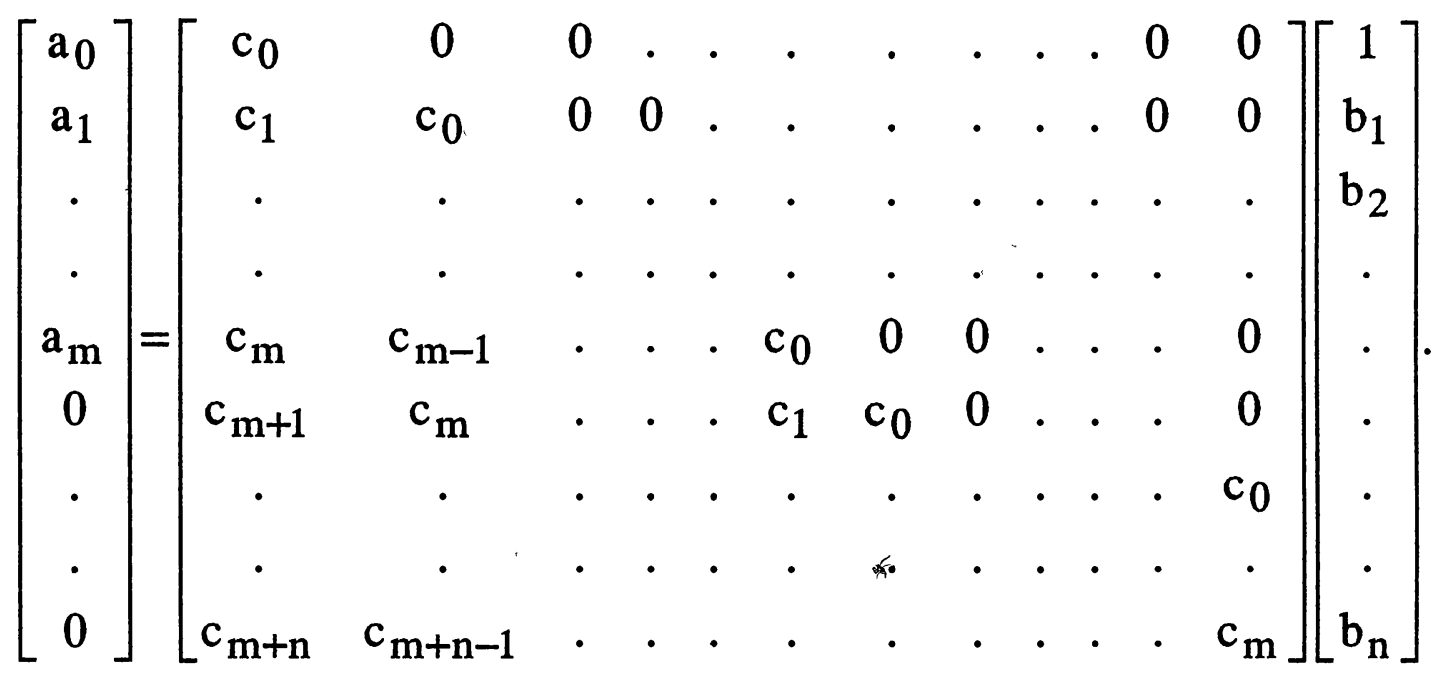

Let us define the new matrices

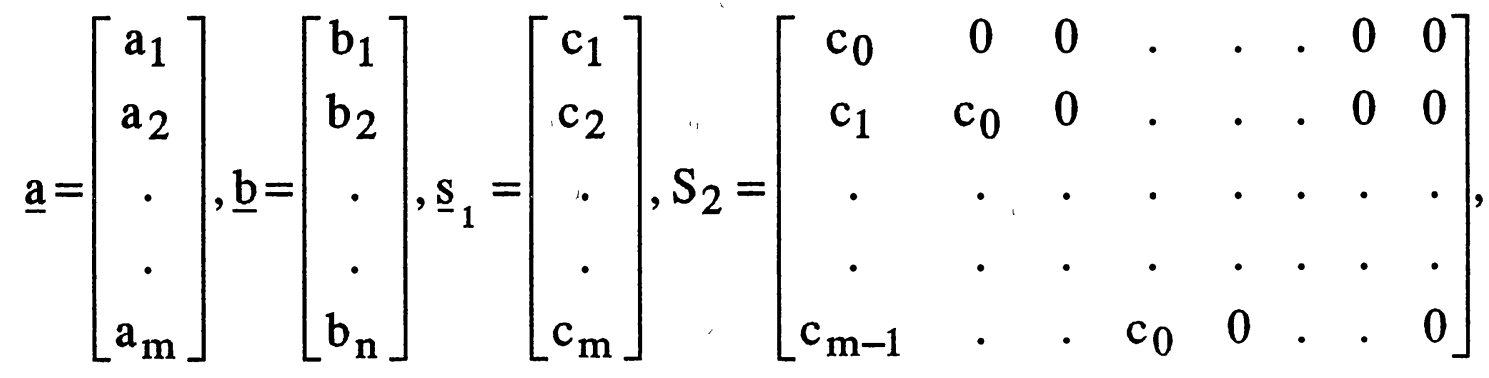


and

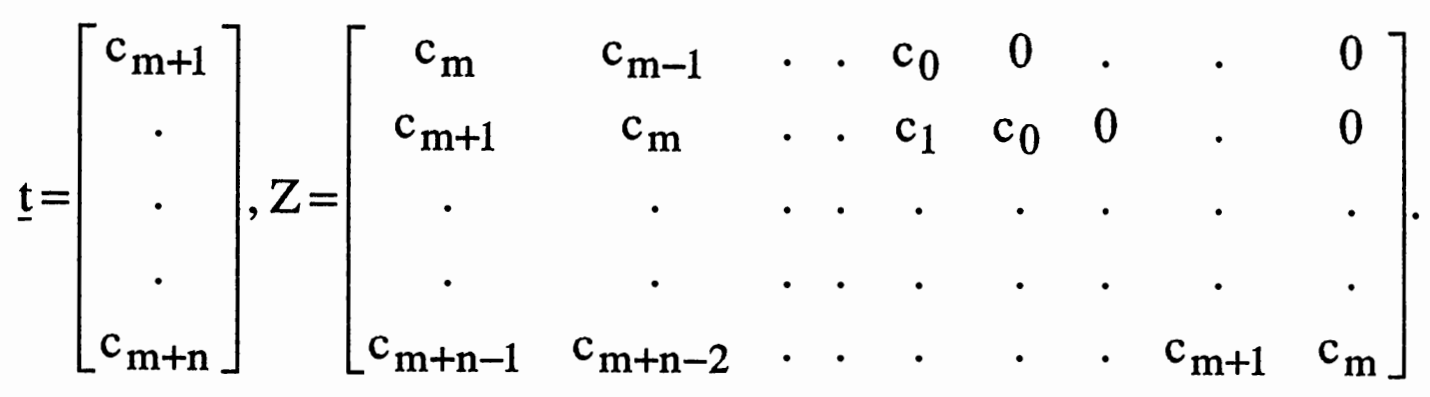

Using (4.31), we can write equation (4.30) as

$$
\left[\begin{array}{c}
\mathrm{a}_{0} \\
\underline{\mathrm{a}} \\
\underline{0}
\end{array}\right]=\left[\begin{array}{cc}
\mathrm{c}_{0} & \underline{0}^{\prime} \\
\underline{s}_{1} & \mathrm{~S}_{2} \\
\underline{t} & \mathrm{Z}
\end{array}\right]\left[\begin{array}{l}
1 \\
\underline{b}
\end{array}\right]
$$

where, prime denotes vector transpose.

The coefficients of $a_{i}$ and $b_{j}$, for $i=0,1, \ldots, m$ and $j=1,2, \ldots, n$, are determined as:

$$
\begin{aligned}
& \mathrm{a}_{0}=\mathrm{c}_{0}=1, \\
& \underline{\mathrm{b}}=-\mathrm{Z}^{-1} \underline{\mathrm{t}}, \\
& \underline{\mathrm{a}}=\underline{\mathrm{s}}_{1}-\mathrm{S}_{2} \mathrm{Z}^{-1} \underline{\mathrm{t}} .
\end{aligned}
$$

\subsubsection{State Space Model}

The Pade approximant models the delay as a finite dimensional dynamic system given by Equation (4.28) where its parameters are determined by Equations (4.33). By referring to Figure 4.6, we may develop the state space model for time delay $\tau$ as follows. 


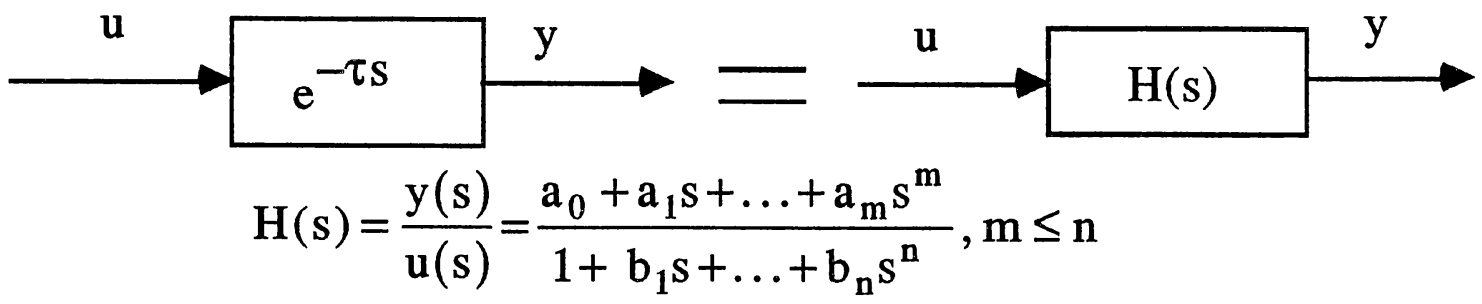

Figure 4.6. Input/output Relationship For Delay $\tau$

$H(s)$ implies the following differential equation

$y(t)+b_{1} \frac{d y(t)}{d t}+\ldots+b_{n} \frac{d^{n} y(t)}{d t^{n}}=a_{0} u(t)+a_{1} \frac{d u(t)}{d t}+\ldots+a_{m} \frac{d^{m} u(t)}{d t^{m}}$

We can choose different state variables to describe the system given in Equation (4.34). Here, a systematic state variable procedure is shown for development of a state space model for the transfer function $\mathrm{H}(\mathrm{s})$ or Equation (4.34). $\mathrm{H}(\mathrm{s})$ may be decomposed into two blocks as.

$$
H(s)=\frac{w(s)}{u(s)} \times \frac{y(s)}{w(s)}=\frac{1}{1+b_{1} s+\ldots+b_{n} s^{n}} \times\left(a_{0}+a_{1} s+\ldots+a_{m} s^{m}\right)
$$

where

$$
\frac{w(s)}{u(s)}=\frac{1}{1+b_{1} s+\ldots+b_{n} s^{n}}, \frac{y(s)}{w(s)}=\left(a_{0}+a_{1} s+\ldots+a_{m} s^{m}\right)
$$

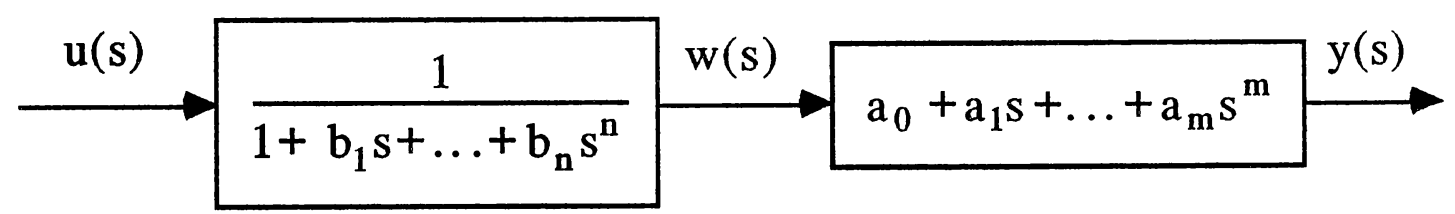


Equation (4.35) implies

$$
\begin{aligned}
& b_{n} s^{n} w(s)=u(s)-\sum_{0}^{n-1} b_{i} s^{i} w(s) \\
& y(s)=a_{0} w(s)+\sum_{1}^{m} a_{i} s^{i} w(s)
\end{aligned}
$$

with the following ordinary differential equations

$$
\begin{aligned}
& \frac{d^{n} w(t)}{d t^{n}}=\frac{1}{b_{n}}\left\{u(t)-\sum_{0}^{n-1} b_{i} \frac{d^{i} w(t)}{d t^{i}}\right\}, \\
& y(t)=a_{0} w(t)+\sum_{1}^{m} a_{i} \frac{d^{i} w(t)}{d t^{i}} .
\end{aligned}
$$

From Equations (4.36), we may set up the following set of first order differential equations by letting $x_{1}=w, x_{i}=\frac{d^{i} w(t)}{d t^{i}}, i=1,2, \ldots, n-1$.

$$
\begin{aligned}
& \dot{x}_{i}=x_{i+1}, i=1,2, \ldots, n-1 \\
& \dot{x}_{n}=\frac{1}{b_{n}} u-\sum_{1}^{n-1} \frac{b_{i}}{b_{n}} x_{i} \\
& y(t)=a_{0} x_{1}(t)+\sum_{1}^{m} a_{i} \dot{x}_{i}
\end{aligned}
$$

The differentiated variables are called the state variables. In vectormatrix notation, Equation (4.37) becomes

$$
\begin{aligned}
& \underline{\dot{x}}_{d}=A_{d} \underline{x}_{d}+\underline{b}_{d} u \\
& y=\underline{c}^{\prime} d \underline{x}_{d}+d_{d} u
\end{aligned}
$$

where 


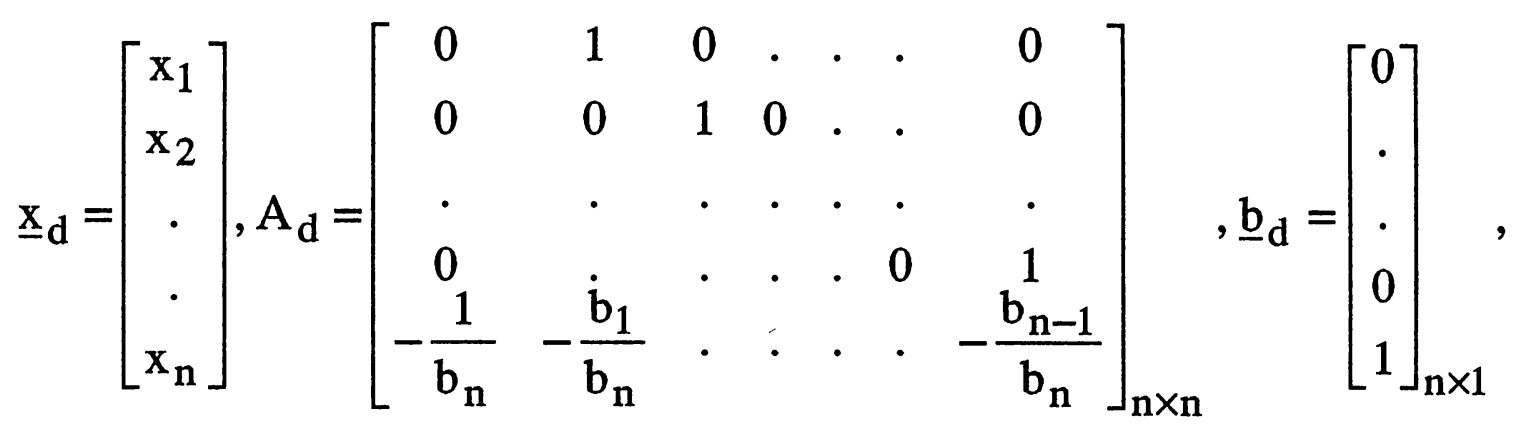

and

$$
\underline{c}_{d}=\left[\begin{array}{c}
a_{0} \\
\cdot \\
a_{m} \\
0 \\
\cdot \\
0
\end{array}\right]_{n \times 1}, d_{d}=\left\{\begin{array}{cc}
0 & \text { if } m<n \\
\frac{a_{n}}{b_{n}} & \text { if } m=n
\end{array}\right\}
$$

In summary, a Pade approximant state space model, Equation (4.38), has been developed for the time delay $\tau$, where Equations (4.33) determine the constant coefficients of the Pade approximant defined in Equation (4.28).

\subsubsection{Solution Of The Modified OCM}

This section replaces the Equations (4.38) for the time delay $\tau$ imbeded within the state equations and solves the OCM problem. Let us assume that we have the controlled system given in Equations (4.1) and (4.3) and the following Pade approximant for the delay $\tau$.

$$
\begin{aligned}
& \underline{\dot{x}}_{d}=A_{d} \underline{x}_{d}+\underline{b}_{d} u, \\
& u_{d}=\underline{c} d \underline{x}_{d}+d_{d} u,
\end{aligned}
$$


where $\underline{x}_{d}$ is a p-dimensional state vector, $A_{d}$ is a square constant matrix of dimension $p, C_{d}$ is a column vector of dimension $p, d_{d}$ is a constant scalar, scalars $u$, and $u_{d}$, are respectively the input and the output of the system. Figure 4.7 represents the equivalent configuration of Figure 4.5 where Equation (4.40) is used for delay $\tau$.

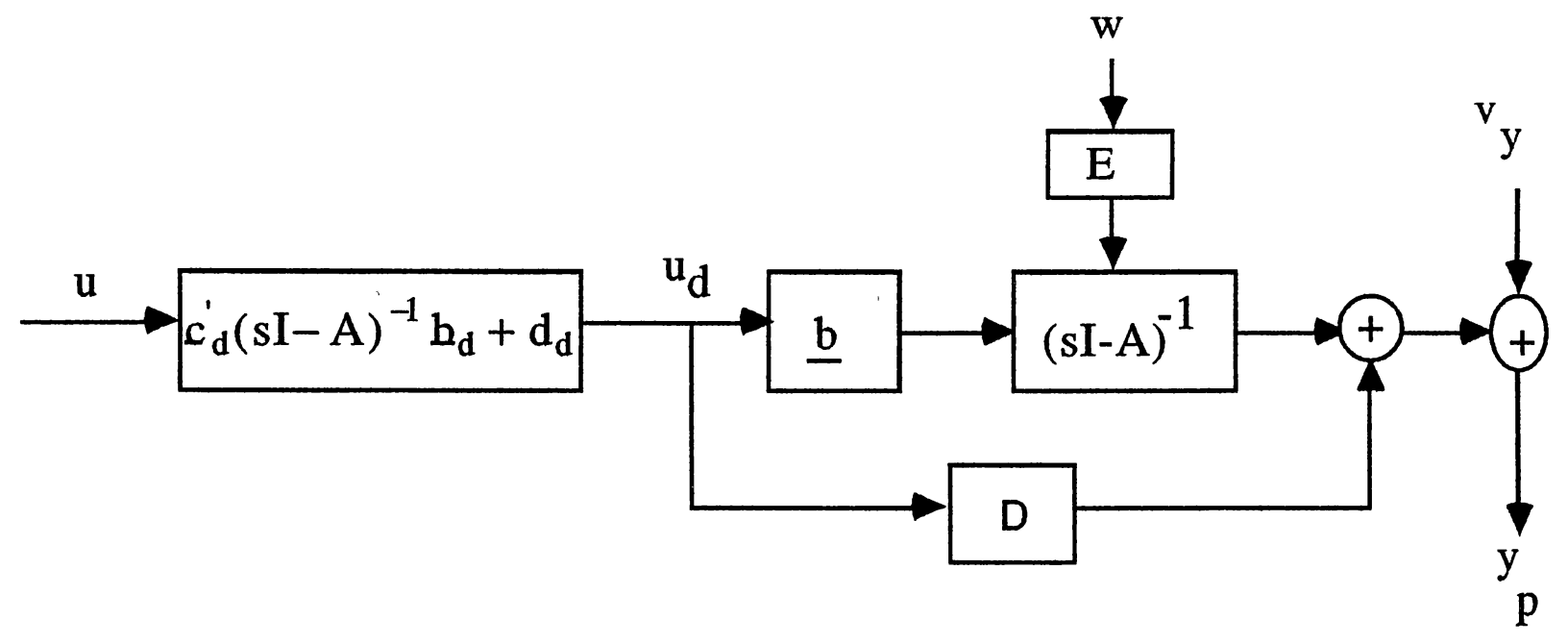

Figure 4.7. Equivalent Configuration Of Figure 4.5

Let us augment the controlled system by the delayed state $\underline{x}_{d}$ and denote the combined state $\underline{x} 1$. The augmented system is represented by

$$
\begin{aligned}
& \underline{\dot{x}}_{1}(t)=\left[\begin{array}{cc}
A_{d} & 0 \\
b \times n \\
b c_{d}^{\prime} & A
\end{array}\right] \underline{x}_{1}+\left[\begin{array}{c}
\underline{b}_{d} \\
\underline{b} d_{d}
\end{array}\right] u+\left[\begin{array}{c}
0_{p \times n_{w}} \\
E
\end{array}\right] \underline{w}(t) \\
& \underline{y}_{p}(t)=\left[\begin{array}{ll}
d \underline{d}_{d}^{\prime} & C
\end{array}\right] \underline{x}_{1}+\underline{d}_{d} d_{d} u+\underline{v}_{y}
\end{aligned}
$$


where

$$
\underline{x}_{1}(t)=\left[\begin{array}{c}
\underline{x}_{d}(t) \\
\underline{x}(t)
\end{array}\right], n_{w}=\text { No. of external state disturbance inputs }
$$

and

$$
\begin{aligned}
& \underline{\dot{x}}(t)=A \underline{x}(t)+\underline{b} u_{d}(t)+E \underline{w}(t) \\
& \underline{y}(t)=C \underline{x}(t)+\underline{d} u_{d}(t) .
\end{aligned}
$$

Let us define the new augmented matrices

$$
\begin{aligned}
& \mathrm{A}_{1}=\left[\begin{array}{cc}
\mathrm{A}_{\mathrm{d}} & 0_{\mathrm{n} \times \mathrm{n}} \\
\underline{\mathrm{b}} \underline{\mathrm{c}}_{\mathrm{d}}^{\prime} & \mathrm{A}
\end{array}\right], \underline{\mathrm{b}}_{1}=\left[\begin{array}{c}
\underline{\mathrm{b}}_{\mathrm{d}} \\
\underline{b}_{\mathrm{d}}
\end{array}\right], \mathrm{E}_{1}=\left[\begin{array}{c}
0_{\mathrm{p} \times \mathrm{n}_{\mathrm{w}}} \\
\mathrm{E}
\end{array}\right], \\
& \mathrm{C}_{1}=\left[\begin{array}{ll}
\underline{\mathrm{d}}_{\underline{c}_{\mathrm{d}}^{\prime}} \mathrm{C}
\end{array}\right], \underline{\mathrm{d}}_{1}=\underline{\mathrm{d}}_{\mathrm{d}} .
\end{aligned}
$$

In terms of the above matrices, we can write the augmented state Equations (4.41) as

$$
\begin{aligned}
& \dot{\mathrm{x}}_{1}(\mathrm{t})=\mathrm{A}_{1} \underline{\mathrm{x}}_{1}+\underline{\mathrm{b}}_{1} \mathrm{u}(\mathrm{t})+\mathrm{E}_{1} \underline{\mathrm{w}}(\mathrm{t}) \\
& \underline{\mathrm{y}}_{\mathrm{p}}(\mathrm{t})=\mathrm{C}_{1} \underline{\mathrm{x}}_{1}(\mathrm{t})+\underline{\mathrm{d}}_{1} u(t)+\underline{\mathrm{v}}_{\mathrm{y}}(\mathrm{t}) .
\end{aligned}
$$

Now, we wish to find an optimal controller for the system (4.43) which minimizes the cost functional of the form

$$
J(u)=E\left\{\underline{x}_{1}^{\prime} Q_{1} \underline{x}_{1}+r u^{2}+g \dot{u}^{2}\right\}
$$

where

$$
\mathrm{Q}_{1}=\left[\begin{array}{cc}
0_{\mathrm{p} \times \mathrm{p}} & 0_{\mathrm{p} \times \mathrm{n}} \\
0_{\mathrm{n} \times \mathrm{p}} & \mathrm{Q}
\end{array}\right]
$$

with $\mathrm{Q}$ is given in Equation (4.4). 
By comparing Equations (4.43) with (4.7), we can see that in this case, there exists no delay between perceived output variables and the state variables (the estimate of $\underline{x}_{1}$ is at time $t$ not at time $t-\tau$ ). Consequently, we have no need of having a predictor for the modified OCM. Hence, for modified OCM, the cascaded combination of the Kalman filter and controller gains are the means by which the human operator optimizes his performance. Figure 4.8 illustrates the modified OCM for the human operator as an optimal controller.

From the proceeding section 4.2.4, we may have the following equations which can be solved for the optimal controller.

$$
\begin{aligned}
& P A_{2}+A_{2}^{\prime} P+Q_{2}-\frac{1}{g} P \underline{b}_{2} \underline{b}_{2}^{\prime} P=0,(n+p) \times(n+p) \\
& F=\frac{1}{g} \underline{b}_{2} P=\left[\begin{array}{llll}
f_{1} & \cdot & f_{n+p} & f_{n+p+1}
\end{array}\right] \\
& \tau_{n}=\frac{1}{f_{n+p+1}} \\
& F^{*}=\left[\begin{array}{llll}
f_{1}^{*} & \cdot & f_{n+p-1}^{*} & f_{n+p}^{*}
\end{array}\right], f_{i}^{*}=\tau_{n} \times f_{i}, i=1,2, \ldots, n+p
\end{aligned}
$$

where

$$
\begin{aligned}
& A_{3}=\left[\begin{array}{cc}
A_{1} & B_{1} \\
0_{1 \times(n+p)} & -\frac{1}{\tau_{n}}
\end{array}\right], B_{n}=\left[\begin{array}{c}
0_{(n+p) \times 1} \\
\frac{1}{t_{n}}
\end{array}\right], C_{3}=\left[\begin{array}{ll}
C_{1} & D_{1}
\end{array}\right] \\
& K=S C_{3}^{\prime} V_{y}^{-1}
\end{aligned}
$$

$$
A_{3} S+S A_{3}^{\prime}+E_{2} W_{2} E_{2}^{\prime}-S C_{3}^{\prime} V_{y}^{-1} C_{3} S=0(n+p+1) \times(n+p+1)
$$

with

$$
\mathrm{W}_{2}=\left[\begin{array}{cc}
\mathrm{W} & 0_{\mathrm{n}_{\mathrm{w}} \times 1} \\
0_{1 \times \mathrm{n}_{\mathrm{w}}} & \frac{\mathrm{V}_{\mathrm{u}}}{\tau_{\mathrm{n}}^{2}}
\end{array}\right], \mathrm{E}_{2}=\left[\begin{array}{cc}
\mathrm{E}_{1} & 0_{(\mathrm{n}+\mathrm{p}) \times 1} \\
0_{1 \times(\mathrm{n}+\mathrm{p})} & 1
\end{array}\right]
$$




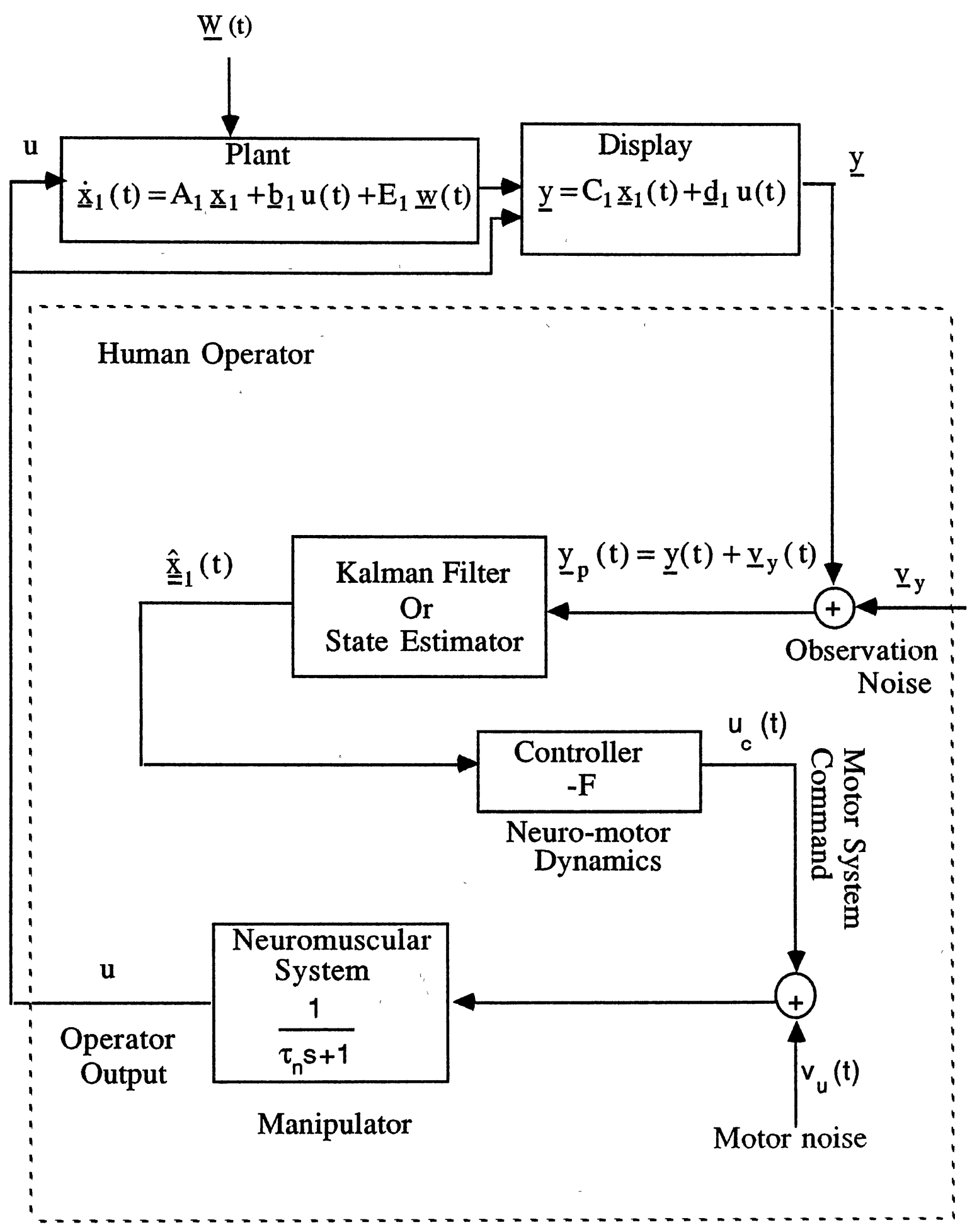

Figure 4.8. Modified OCM Structure 


\subsection{Model Validation}

We are given the variables $\mathrm{A}, \underline{\mathrm{b}}, \mathrm{C}, \underline{\mathrm{d}}$, and $\mathrm{E}$ which characterize the behavior of the controlled system given in Equations (4.1) and (4.3) and we assume that the cost weightings in the index of performance J, Equation (4.4), are known.

In order to implement the OCM, we have to know the human response parameters $\tau, \tau_{\mathrm{n}}, \mathrm{V}_{\mathrm{y}}$, and $\mathrm{V}_{\mathrm{u}}$. As mentioned earlier, there are reasonable approximations for the effective time delay $\tau=0.15$ $0.25 \mathrm{~s}$ and for the neuromotor lag $\tau_{\mathrm{n}}=0.1 \mathrm{~s}$. For a given $\tau_{\mathrm{n}}$, we can simply determine a control rate weighting, $g$, in Equation (4.4) so that the corresponding control gain matrix $F$ determined by Equations (4.9)-(4.12) yields $\tau_{\mathrm{n}}$ as required.

$$
\tau_{n}=\frac{1}{f_{n+1}}
$$

Once the values of $\tau$ and $g$ are specified, starting with initial guesses for intensities $V_{y}$ and $V_{u}$, one may proceed with an iterative procedure to adjust $V_{y}$ and $V_{u}$ in such a way that

$$
\begin{aligned}
& \frac{\mathrm{V}_{\mathrm{y}_{\mathrm{il}}}}{\Pi E\left\{\mathrm{y}_{\mathrm{i}}^{2}(\mathrm{t})\right\}}=\rho_{\mathrm{y}_{\mathrm{i}}}, \quad \mathrm{i}=1,2, \ldots, \mathrm{m} \\
& \frac{\mathrm{V}_{\mathrm{u}}}{\Pi E\left\{\mathrm{u}_{\mathrm{c}}^{2}(\mathrm{t})\right\}}=\rho_{\mathrm{u}}
\end{aligned}
$$

where the noise-to-signal ratios $\rho_{\mathrm{u}}$ and $\rho_{\mathrm{y}_{1}}$ have been provided from empirical data, and their typical values are 0.003 and 0.01 respectively. 
After adjusting the intensities $V_{y}$ and $V_{u}$, we are ready to apply the OCM model to predict different facets of human behavior. For example, the closed loop human performance can be predicted by Equations (4.24)-(4.26). A computer program which implements the OCM has been developed [8].

Kleinman et al. [55] have shown that their optimal control model works well for both simple and complex tracking tasks. In simple tracking, they considered three sets of plant dynamics $\mathrm{K}, \frac{\mathrm{k}}{\mathrm{s}}, \frac{\mathrm{k}}{\mathrm{s}^{2}}$ in validation experiments. The state disturbance was a sum of 13 sinusoids whose amplitudes were selected to simulate a first order noise spectrum having a break frequency of $2 \mathrm{rad} / \mathrm{s}$; the noise is applied as a velocity disturbance to the plant as depicted in Figure 4.9. The subjects were instructed to minimize the following mean square index of performance

$$
J(u)=E\left\{e^{2}\right\}+g E\left\{\dot{u}^{2}\right\}
$$

The values of $\tau, \tau_{\mathrm{n}}, \mathrm{V}_{\mathrm{y}}$ and $\mathrm{V}_{\mathrm{u}}$ were computed from matching empirical data as mentioned before. Nominal values of $\tau$ and $\tau_{\mathbf{n}}$ were 0.15 and 0.1 second, respectively. The value of control rate in (4.45), $\mathrm{g}=0.00017$, was adjusted by the selection of $\tau_{\mathbf{n}}=0.1 \mathrm{~s}$. After several optimization iterations, the following values of intensities of $\mathrm{V}_{\mathrm{y}}$ and $\mathrm{V}_{\mathbf{u}}$ were chosen,

$$
\mathrm{V}_{\mathrm{u}}=0.048143, \mathrm{~V}_{\mathrm{y}}=\left[\begin{array}{cc}
0.0037051 & 0 \\
0 & 0.0968629
\end{array}\right]
$$


with signal-to-noise ratios

$$
\rho_{\mathrm{u}}=-25.0002 \mathrm{~dB} \text { and } \rho_{\mathrm{y}}=\left[\begin{array}{l}
-20.0002 \\
-20.0003
\end{array}\right] \mathrm{dB} \text {. }
$$

Figure 4.10 shows a comparison of the frequency-response plots of the measured and predicted results for simple tracking $Y_{c}=1 / s$. As seen, they agree with the empirical data completely. Table 4.1 also compares measured and predicted values of the variances of error, error rate and control input.

\subsubsection{An Example of Aircraft Landing Approach}

The following example illustrates the application of the (modified) OCM in controlling aircraft during landing and shows the qualitative comparison of the OCM with the Cross-Over model for the human operator (pilot).

Figure 4.11 shows a block-diagram representation of pilotvehicle system for this example. This is an example of controlling aircraft against wind gust during landing approach. The external input is injected as though there were a disturbance noise such as gust wind acting on the aircraft. The vehicle dynamic, $H_{c}$, is such that the human operator (pilot) needs to provide some amount of lead compensation.

The values for $\tau$ and $\tau_{\mathrm{n}}$ are chosen as $0.15 \mathrm{~s}$ and $01 \mathrm{~s}$, respectively. The values of control rate factor, $g=3.98 \times 10^{-4}$, and intensities $\mathrm{V}_{\mathrm{u}}=0.9826, \mathrm{~V}_{\mathrm{y}}=0.2174$ are adjusted so as to produce $\tau_{\mathrm{n}}=0.1 \mathrm{~s}$ and noise-to-signal ratios $\rho_{\mathrm{u}}=-25.0006 \mathrm{~dB}, \rho_{\mathrm{y}}=-20.0004 \mathrm{~dB}$, respectively. 


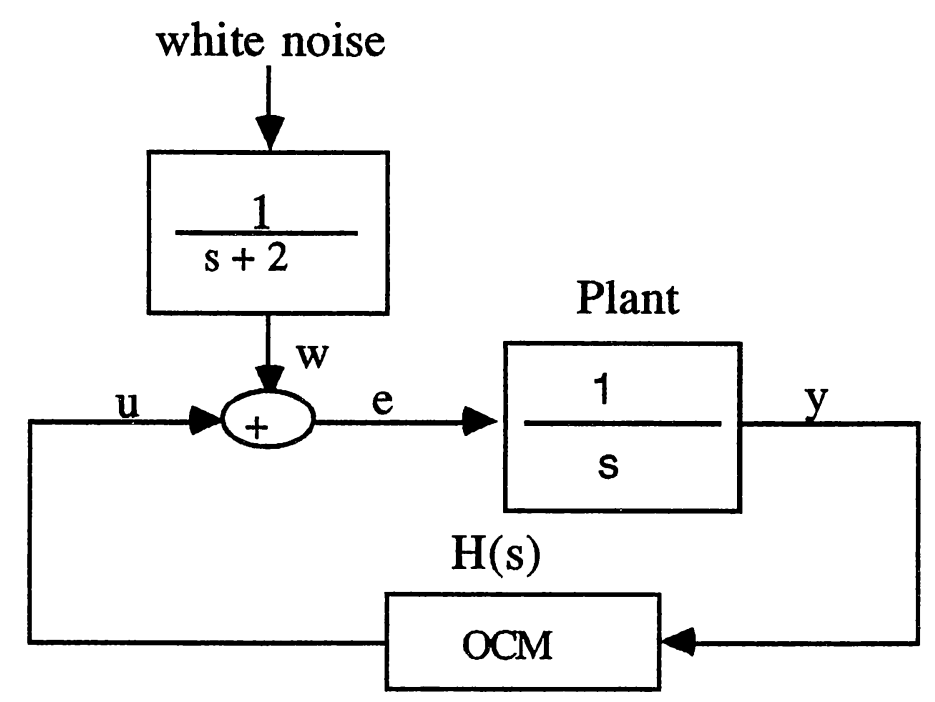

Figure 4.9. Block Diagram of Example One of Ref. [55]

TABLE 4.1

MEASURED AND THEORETICAL MEAN SQUARE VALUES OF ERROR, ERROR RATE AND CONTROL INPUT. FROM TABLE 1 REFERENCE [55].

\begin{tabular}{c|lll|lll|l}
\hline \multicolumn{2}{c}{ M. S. Error } & & \multicolumn{2}{c}{ M. S. Error Rate } & & \multicolumn{2}{c}{ M. S. Control } \\
\cline { 6 - 7 } \cline { 5 - 7 } Meas. & Theor. & & Meas. & Theor. & & Meas. & Theor. \\
0.13 & 0.12 & & 3.1 & 3.06 & & 4.2 & 3.83 \\
\hline
\end{tabular}




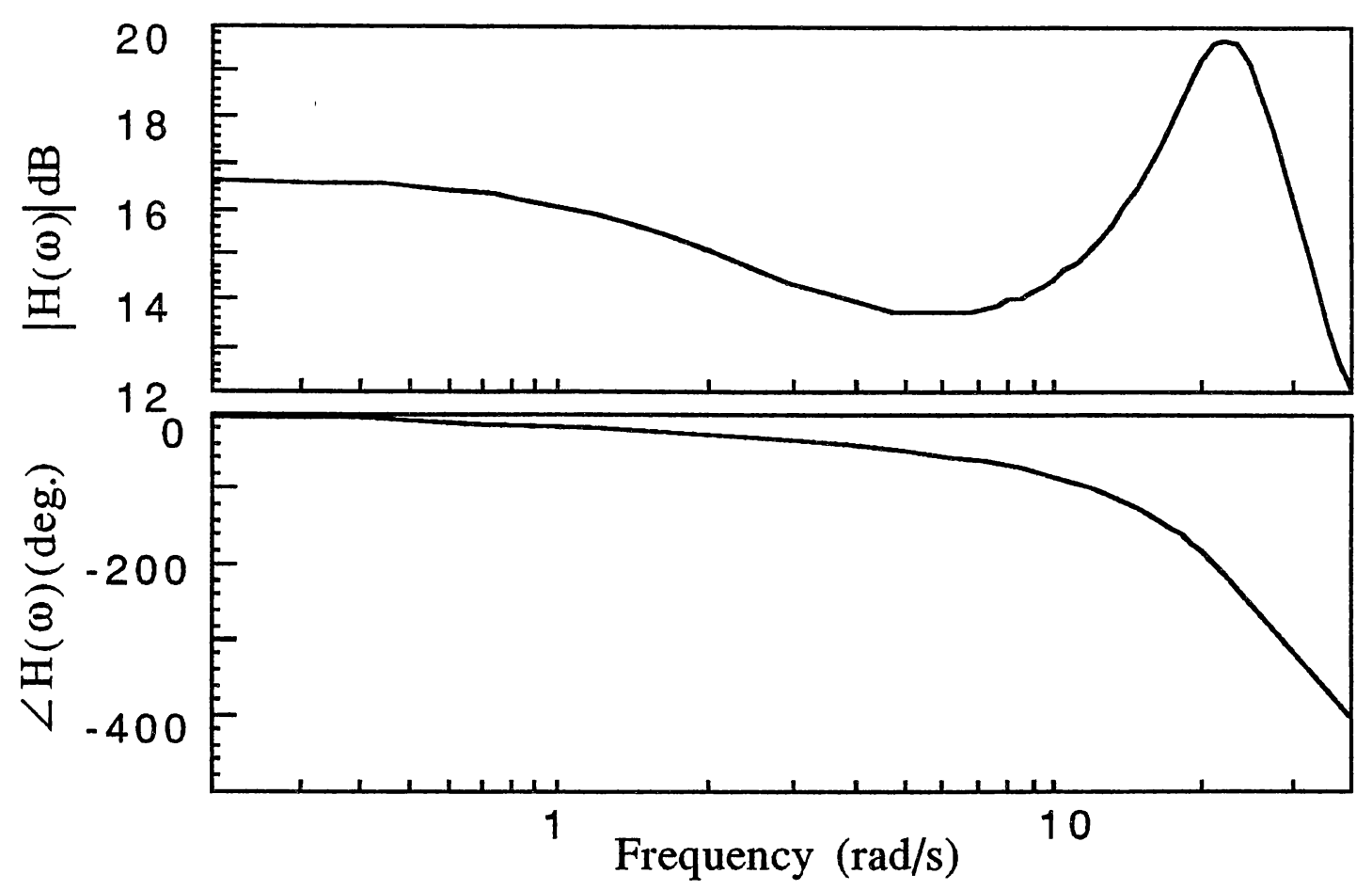

a. Results Using Pade Approximation
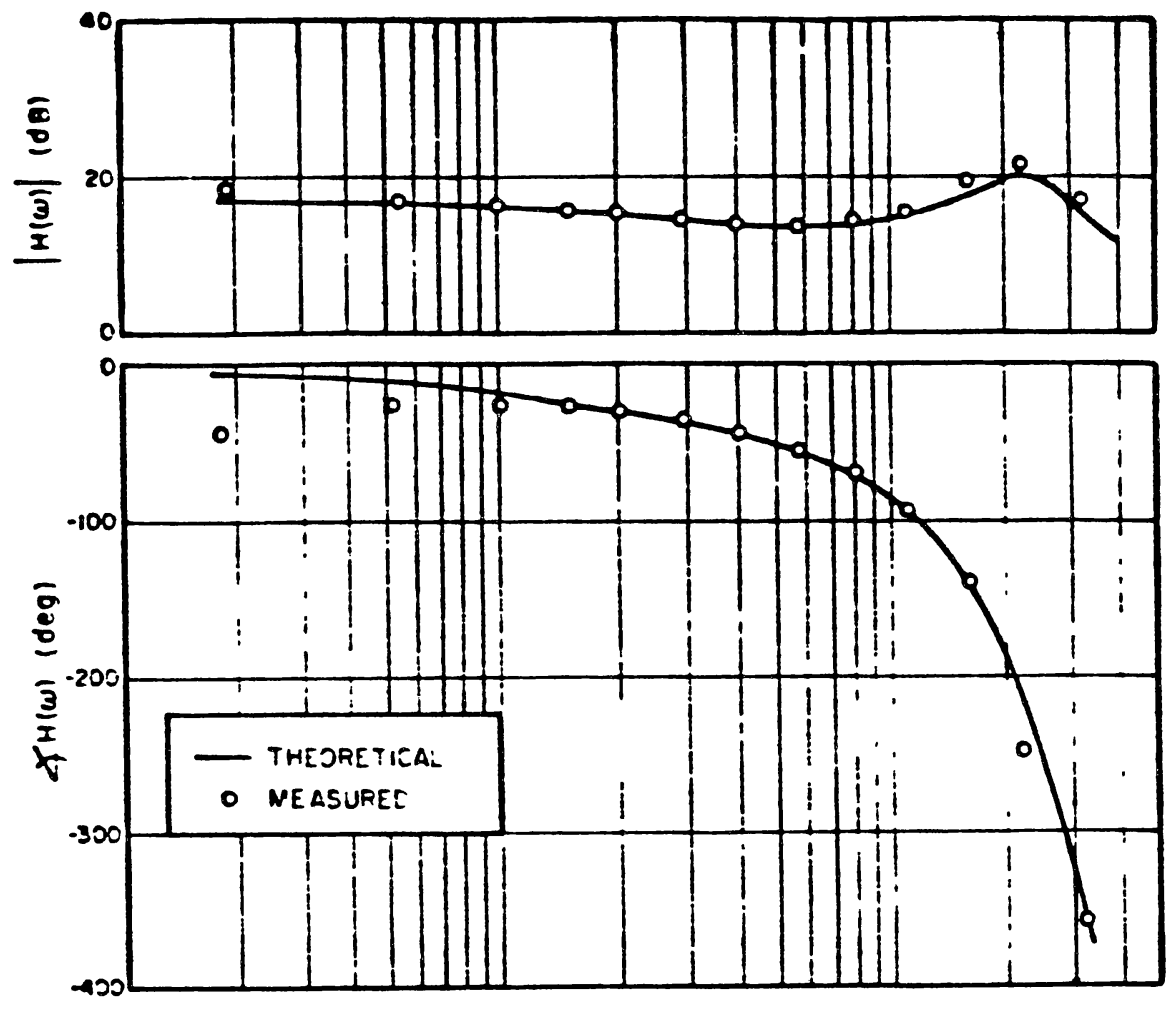

FIGURE 4.10. Experimental Validation Of KBL's Model in a simple Tracking Task, $\mathrm{Y}_{\mathrm{c}}=1 / \mathrm{s}$. 


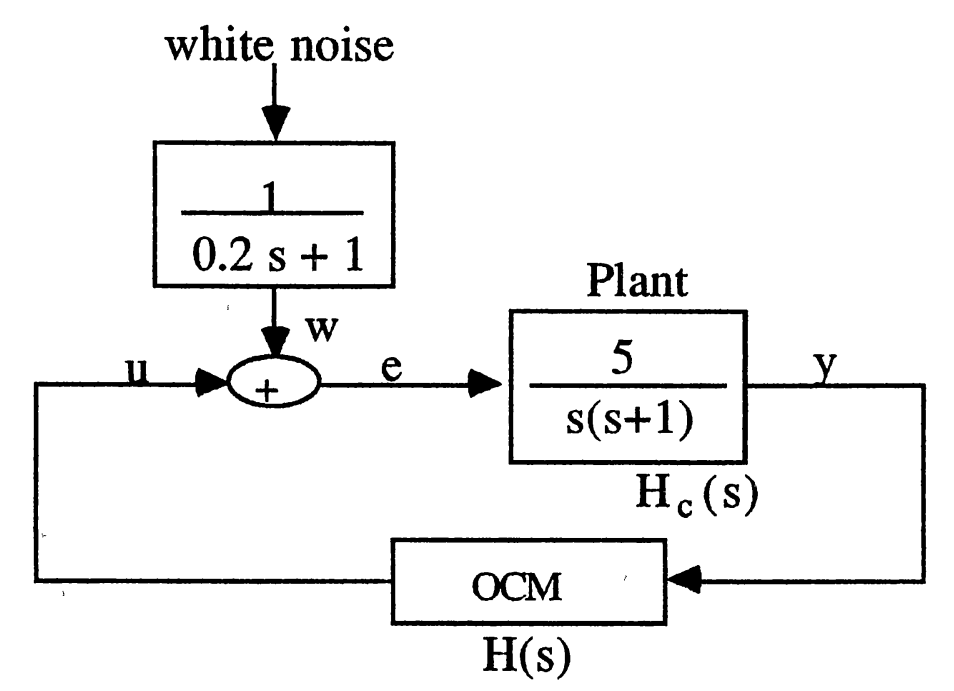

Figure 4.11. Controlling Aircraft Against Wind Gust During Landing

The frequency response (magnitude $\&$ phase) plots of the modified OCM solution for the open loop transfer function $\mathrm{G}=\mathrm{H} \times \mathrm{H}_{\mathrm{c}}$ are depicted in Figure 4.12, where $\mathrm{H}$ is the pilot transfer function linking $y$ to $u$. The transfer function $G$, represents approximately $\propto \frac{1}{S}$ form in the cross-over region and a resonant peak (not explicitly shown in Figure 4.12) and a high frequency effective time delay resulting from the dynamics of the Pade approximation used for delay $\tau$. As seen, the OCM yields a solution that may be compared qualitatively with the Cross-Over model, but with different dynamic modes. 
The following transfer function has been obtained for the human operator (pilot) in Figure 4.11.

$$
H(s)=\frac{(s+5)(s+5.3809)(s+10.0255)(s+13.33)}{(s+4.8904)(s+5)(s+10.0076)(s+18.8807)\left(s^{2}+11.435 s+154.13\right)}
$$

As seen, the OCM tends to have near and exact pole \& zero concellations. Figure 4.13 shows that the human operator, as an optimal controller, acts like a lead compensator for the vehicle, as illustrated in Figure 4.11.
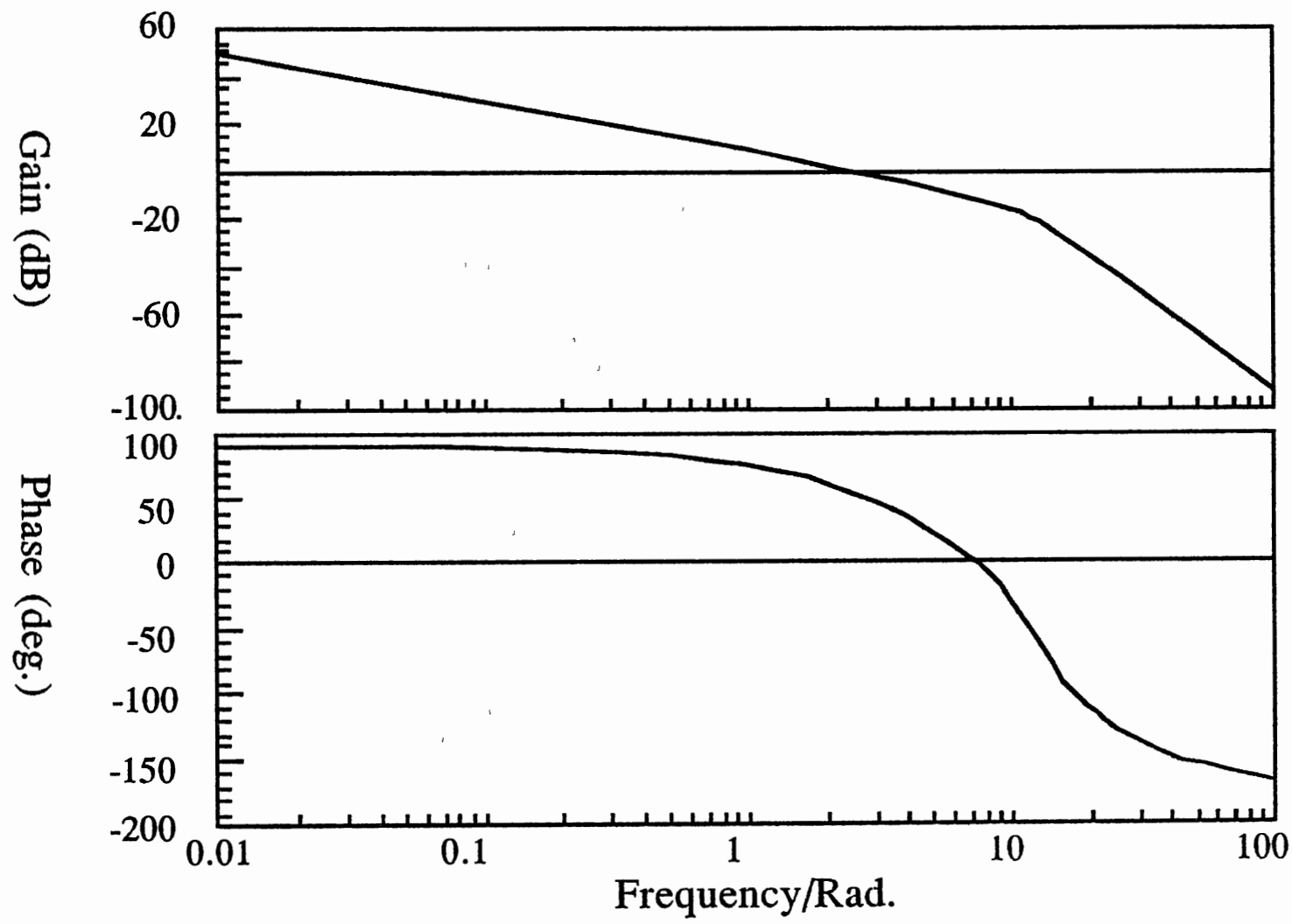

Figure 4.12. Frequency Plots For Open Loop System Figure 4.11 

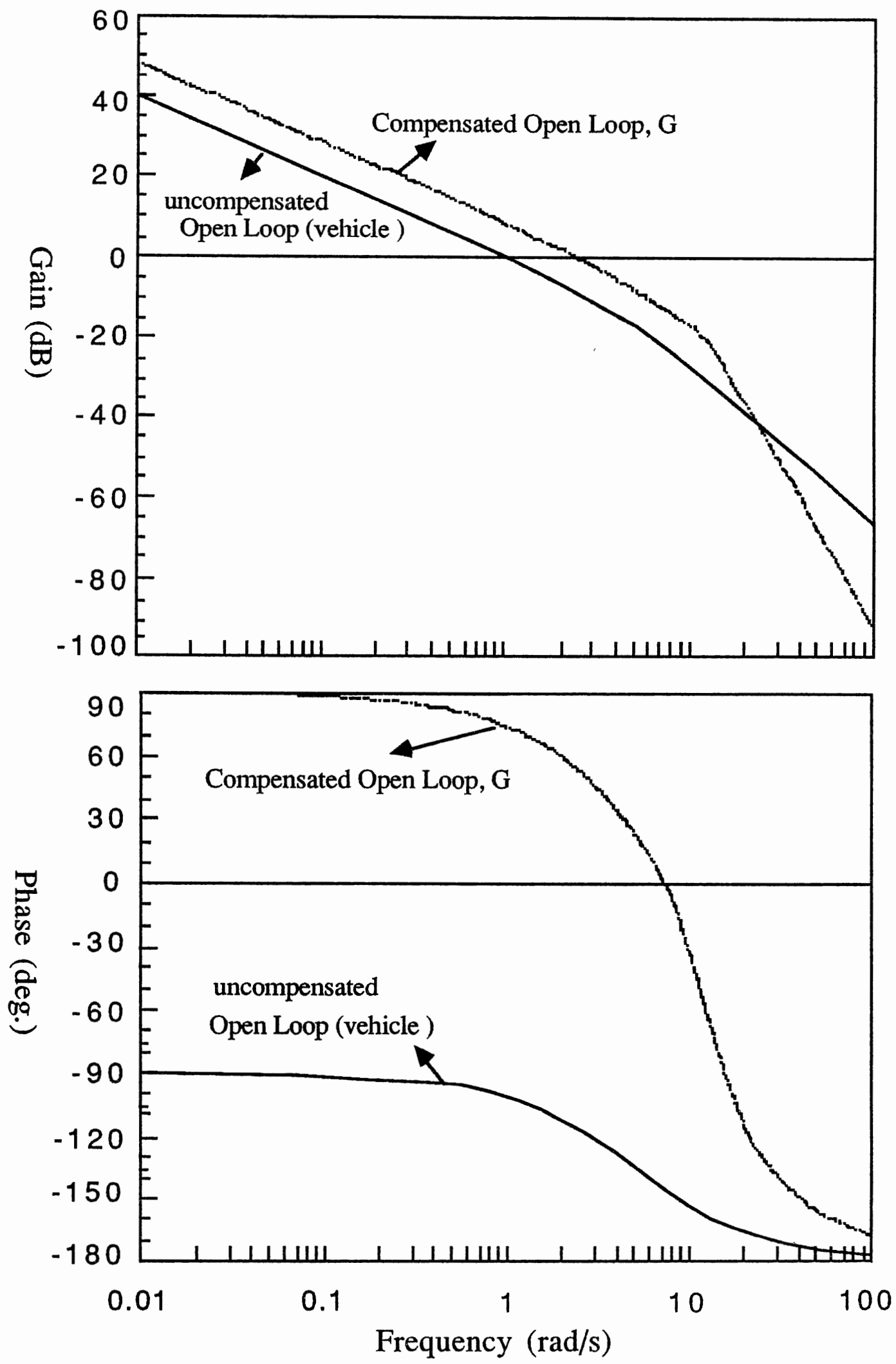

Figure 4.13. The Human operator As a Phase Lead Compensator 
The effect of the Pade approximant model used for time delay $\tau$ on human performance is illustrated in Figure 4.14. As the order of Pade approximant increases, the resonant peak will increase.

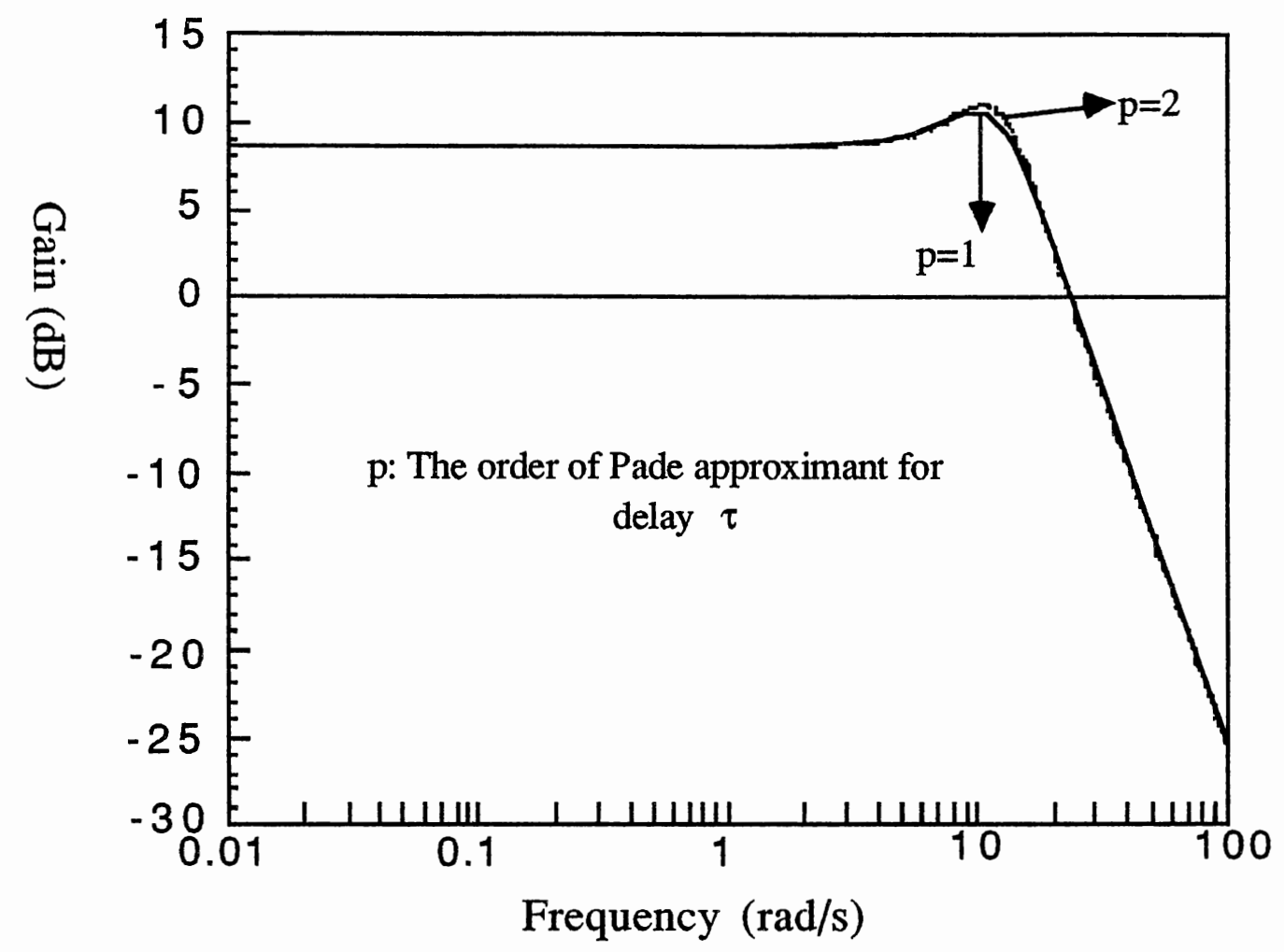

Figure 4.14. Resonant Peak In Human Operator Transfer Function

\subsection{Summary}

In this chapter we considered the Optimal Control Model (OCM) as a powerful tool in predicting human performance in manuallycontrolled sytems. We saw that the objective of the OCM is to 
maximize the adaptive characteristics of the human operator subjected to his own constraints, which are represented by visual and motor lag delays, and by some sources of remnants (motor and observation noises).

The Kleinman-Baron-Levison model of the human operator, known as the standard OCM, has been discussed in section 4.2. This section first outlines the general description, main assumptions and basic structure of the OCM and then presents the solution of the OCM in time domain as well as in frequency domain. It has been concluded that the OCM (as a cascaded combination of three key elements, Kalman filter, minimum mean square predictor, and optimal controller gain factor) is completely determined by the human operator's inherent limitations ( time delays and intensities of the observation and motor noise).

Section 4.3 considered the modofied OCM which has the basic structure of the standard OCM, except that the delay elements are modeled as finite order dynamic systems by using the Pade approximant technique. Hence, for the modified OCM, the delays are imbedded within the state equations, and there is no need of having a predictor. Section 4.3 also presents the solution of the modified OCM. It has been shown that the cascaded combination of the Kalman filter and the controller gains are the means by which the human operator optimizes his performance. Finally, the OCM model validations have been discussed in section 4.4 . 


\section{CHAPTER V}

\section{DUAL OCM}

\subsection{Introduction}

As discussed in the preceding chapter, because of its predictive capability, the OCM has been used as a powerful analytical tool to predict and analyze the closed-loop performance of man-machine systems for well-trained operators. Some of the applications of the OCMs are listed below.

1 - Display design \& evaluation [74-77]

2 - Prediction of the pilot ratings of aircraft handling qualities [79]

3 - Supervision of several semi-automated subsystems [71]

4- Workload estimation [78]

5 - Simulator design and flight simulation tasks $[2,4,7]$

6- Networked simulator problems [8]

This chapter presents one of the applications of the OCM in a networked aircraft simulator problem (Formation Flight Task). The general problem in networking multiple simulators is the determination of the maximum amount of inter-simulator communication time delay. Moreover, it has been shown [8] that the network delays are problematic in any of the following networked flight simulator tasks. 
i. Formation flight

ii. Air-to-air combat

iii. Air-to-air refueling

iv. Target handoff

This chapter considers the formation flight task developed in [8]. The effects of network delays on a simplified formation flight task will be discussed in chapter VI. The formation flight task is formulated as a Dual OCM model which is fully discussed in the next section.

\subsection{The Dual OCM and its Solution}

This section presents the solution of the dual OCM for Formation Flight tasks. Figure 5.1 shows a block diagram representation of the dual OCM. As indicated in Figure 5.1, there are two aircraft; one is a Lead pilot who maintains the level of flight while experiencing turbulence (e.g., wind gust), and one is a Wingman who is attempting to mimic the lead pilot while being affected by the same turbulence, $w(t)$. Here, it is assumed that the first OCM (lead pilot) does not need to interact with the second OCM (Wingman). Therefore, the first OCM can be developed independently as discussed in the previous chapter. 


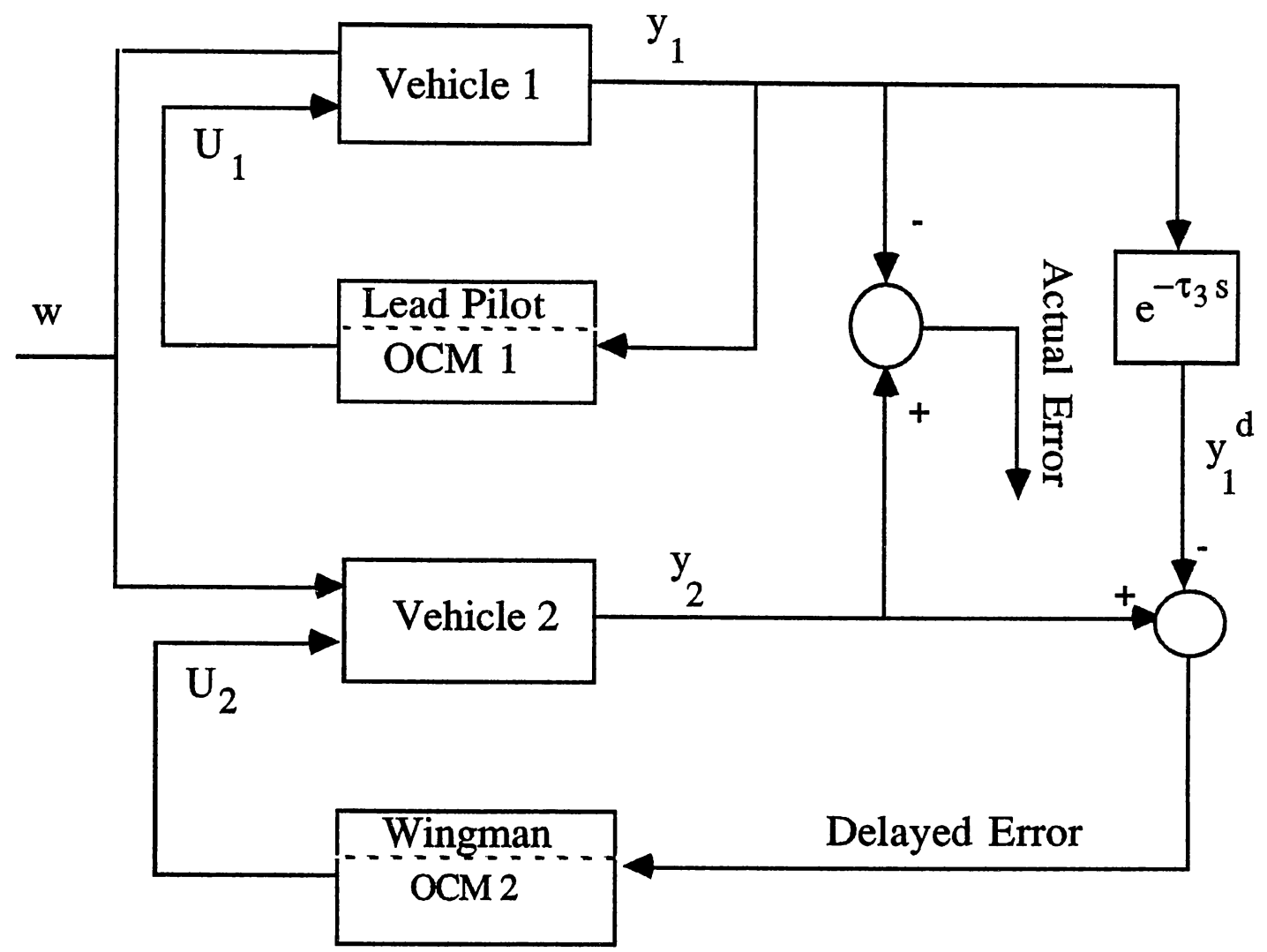

Figure 5.1. Dual OCM STructure for Formation Flight

\subsubsection{OCM1 Development}

We assume that the operation of the first vehicle is described by the following time-invariant linear equations

$$
\begin{aligned}
& \underline{\underline{x}}_{1}(t)=A_{1} \underline{x}_{1}(t)+\underline{b}_{1} u_{d_{1}}(t)+E_{1} \underline{w}_{1}(t) \\
& \underline{y}_{1}(t)=C_{1} \underline{x}_{1}(t)+\underline{d}_{1} u_{d_{1}}(t)
\end{aligned}
$$

and the Lead pilot perceives the variables

$$
\begin{aligned}
\underline{y}_{\dot{p}_{1}}(t)=\underline{y}_{1}(t)+\underline{v}_{y_{1}}(t)= & \\
& C_{1} \underline{x}_{1}(t)+\underline{d}_{1} u_{d_{1}}(t)+\underline{v}_{y_{1}}(t)
\end{aligned}
$$


It is assumed that we have the following Pade approximation for the time-delay $\tau_{1}$.

$$
\begin{aligned}
& \underline{\dot{x}}_{d_{1}}(t)=A_{d_{1}} \underline{x}_{d_{1}}(t)+\underline{b}_{d_{1}} u_{1}(t) \\
& u_{d_{1}}(t)=C_{d_{1}} \underline{x}_{d_{1}}(t)+\underline{d}_{d_{1}} u_{1}(t)
\end{aligned}
$$

where

$\underline{x}_{1}-n_{x_{1}}$-dimensional state vector

$\mathbf{u}_{1}$ - scalar

$\mathrm{u}_{\mathrm{d}_{1}}-$ scalar

$\underline{w}$ - $n_{w}$-dimensional column vector

$\mathrm{y}_{1}-\mathrm{n}_{\mathrm{y}_{1}}$-dimensional output vector

$\underline{x}_{d_{1}}-n_{x_{d_{1}}}$-dimensional state vector

$A_{1}-n_{x_{1}} \times n_{x_{1}}$-dimensional constant matrix

$\underline{b}_{1}-n_{x_{1}}-$ dimensional column vector

$\mathrm{E}_{1}-\mathrm{n}_{\mathrm{x}_{1}} \times \mathrm{n}_{\mathrm{w}}-$ dimensional constant matrix

$\mathrm{C}_{1}-\mathrm{n}_{\mathrm{y}_{1}} \times \mathrm{n}_{\mathrm{x}_{1}}$-dimensional constant matrix

$\underline{\mathrm{d}}_{1}-\mathrm{n}_{\mathrm{y}_{1}}$-dimensional constant column vector

$\underline{b}_{d_{1}}-n_{x_{d_{1}}}$ dimensional constant column vector

$A_{d_{1}}-n_{x_{d_{1}}} \times n_{x_{d_{1}}}$-dimensional constant matrix

$\mathrm{C}_{\mathrm{d}_{1}}-\mathbf{n}_{\mathrm{x}_{\mathrm{d}_{1}}}$-dimensional constant row vector

$d_{d_{1}}-$ scalar

$\underline{w}(t)$ and $\underline{v}_{y_{1}}$ are white Gaussian noise with covariances

$$
\begin{aligned}
& E\left\{w_{i}(t) w_{j}(\tau)\right\}=\left\{\begin{array}{lll}
w_{i} \delta(t-\tau) \text { if } & i=j \\
0 & \text { if } & i \neq j
\end{array}\right\} \\
& E\left\{\underline{v}_{y_{1_{i}}}(t) \underline{v}_{y_{1_{j}}}^{\prime}(\tau)\right\}=\left\{\begin{array}{cccc}
V_{y_{i}} \delta(t-\tau) & \text { if } & i=j \\
0 & \text { if } & i \neq j
\end{array}\right\}
\end{aligned}
$$


$\underline{\mathrm{v}}_{\mathrm{y}_{1}}$ denotes the $\mathrm{i}$-th component of the vector $\underline{\mathrm{v}}_{\mathrm{y}_{1}}$.

or

$$
\begin{aligned}
& \mathrm{E}\left\{\underline{\mathrm{w}}(\mathrm{t}) \underline{\mathrm{w}}^{\prime}(\tau)\right\}=\mathrm{W} \delta(\mathrm{t}-\tau), \quad \mathrm{W}=\left[\begin{array}{ccccc}
\mathrm{w}_{1} & 0 & \cdot & \cdot & 0 \\
0 & \mathrm{w}_{2} & 0 & \cdot & 0 \\
\cdot & 0 & \cdot & \cdot & \cdot \\
\cdot & \cdot & \cdot & \cdot & 0 \\
0 & \cdot & \cdot & 0 & \mathrm{w}_{\mathrm{n}_{\mathrm{w}}}
\end{array}\right]_{\left(\mathrm{n}_{\mathrm{w}} \times \mathrm{n}_{\mathrm{w}}\right)} \\
& \mathrm{E}\left\{\underline{\mathrm{v}}_{\mathrm{y}_{1}}(\mathrm{t}) \underline{\mathrm{v}}_{\mathrm{y}_{1}}^{\prime}(\tau)\right\}=\mathrm{V}_{\mathrm{y}_{1}} \delta(\mathrm{t}-\tau), \quad \mathrm{V}_{\mathrm{y}_{1}}=\left[\begin{array}{ccccc}
\mathrm{v}_{\mathrm{y}_{1}} & 0 & \cdot & \cdot & 0 \\
\cdot & \mathrm{v}_{\mathrm{y}_{2}} & 0 & \cdot & 0 \\
\cdot & 0 & \cdot & \cdot & \cdot \\
0 & \cdot & \cdot & \cdot & 0 \\
0 & \cdot & \cdot & 0 & \mathrm{v}_{\mathrm{y}_{\mathrm{n} 1}}
\end{array}\right]_{\left(\mathrm{n}_{\mathrm{y}_{1}} \times \mathrm{n}_{\mathrm{y}_{1}}\right)}
\end{aligned}
$$

Further, it is assumed that the lead pilot tries to minimize the index of performance

$$
J\left(u_{1}\right)=E\left\{\underline{x}_{1}^{\prime}(t) Q_{1} \underline{x}_{1}(t)+g_{1} \dot{u}_{1}^{2}(t)\right\}
$$

where $\mathrm{Q}_{1}$ is a nonnegative definitive symmetric matrix. The first term in (5.6) represents the mean square error and the second term represents the mean square of the control rate.

By substituting (5.5) into (5.1) and (5.2), we will have the following equations

$$
\begin{aligned}
& \underline{\dot{x}}_{1}(t)=A_{1} \underline{x}_{1}(t)+\underline{b}_{1} C_{d_{1}} \underline{x}_{d_{1}}(t)+\underline{b}_{1} d_{d_{1}} u_{1}(t)+E_{1} \underline{w}(t) \\
& \underline{y}_{1}(t)=C_{1} \underline{x}_{1}(t)+\underline{d}_{1} C_{d_{1}} \underline{x}_{d_{1}}(t)+\underline{d}_{1} d_{d_{1}} u_{1}(t) .
\end{aligned}
$$

Let us define the following new quantities 


$$
\begin{aligned}
& \underline{x}_{3}(t)=\left[\begin{array}{c}
\underline{x}_{d_{1}}(t) \\
\underline{x}_{1}(t)
\end{array}\right], \quad A_{3}=\left[\begin{array}{cc}
A_{d_{1}} & 0 \\
\underline{b}_{1} C_{d_{1}} & \left.A_{x_{d_{1}}} \times n_{x_{1}}\right)
\end{array}\right], \quad \underline{b}_{3}=\left[\begin{array}{c}
\underline{b}_{d_{1}} \\
\underline{b}_{1} d_{d_{1}}
\end{array}\right], \\
& E_{3}=\left[\begin{array}{c}
0 \\
\left(n_{\mathrm{n}_{d_{1}}} \times 1\right) \\
E_{1}
\end{array}\right], \quad C_{3}=\left[\begin{array}{ll}
\underline{d}_{1} C_{d_{1}} & C_{1}
\end{array}\right], \quad \underline{d}_{3}=\underline{d}_{1} d_{d_{1}} .
\end{aligned}
$$

In terms of the above augmented state and matrices, the Equations (4.7) and (4.8) are rewritten as

$$
\begin{aligned}
& \underline{\dot{x}}_{3}(t)=A_{3} \underline{x}_{3}(t)+\underline{b}_{3} u_{1}(t)+E_{3} \underline{w}(t) \\
& \underline{y}_{1}(t)=C_{3} \underline{x}_{3}(t)+\underline{d}_{3} u_{1}(t) .
\end{aligned}
$$

The performance index $J(u)$, Equation (4.6), becomes

$$
J\left(u_{1}\right)=E\left[\underline{x}_{2}^{\prime}(t) Q_{2} \underline{x}_{2}(t)+g_{1} \dot{u}_{1}^{2}(t)\right]
$$

where

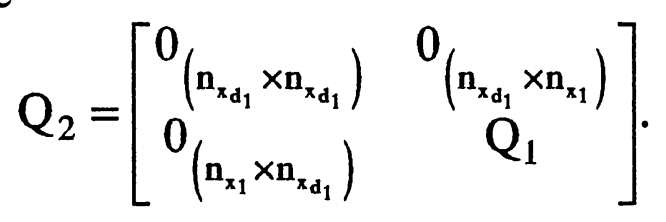

Now, we augment the state $\underline{x}_{3}$ by $\mathbf{u}_{1}$ and denote the new state $\underline{\mathrm{x}}_{4}(\mathrm{t})$,

$$
\underline{\mathrm{x}}_{4}(\mathrm{t})=\left[\begin{array}{l}
\underline{\mathrm{x}}_{3}(\mathrm{t}) \\
\mathrm{u}_{1}(\mathrm{t})
\end{array}\right]
$$

In terms of $\underline{x}_{4}(t)$, Equations (5.9) and (5.11) become

$$
\begin{aligned}
& \underline{\dot{x}}_{4}(\mathrm{t})=\mathrm{A}_{4} \underline{\mathrm{x}}_{4}(\mathrm{t})+\underline{\mathrm{b}}_{4} \mu(\mathrm{t})+\mathrm{E}_{4} \underline{\mathrm{w}} \\
& \mathrm{J}\left(\mathrm{u}_{1}\right)=\mathrm{E}\left\{\underline{\mathrm{x}}_{4}^{\prime}(\mathrm{t}) \mathrm{Q}_{4} \underline{\mathrm{x}}_{4}(\mathrm{t})+\mathrm{g}_{1} \mu_{1}^{2}\right\}
\end{aligned}
$$


where

$$
A_{4}=\left[\begin{array}{cc}
A_{3} & \underline{b}_{3} \\
0 & 0 \\
1 \times\left(n_{x_{1}}+n_{x_{d_{1}}}\right) & 0
\end{array}\right], \underline{b}_{4}=\left[\begin{array}{c}
0 \\
\left(n_{x_{1}}+n_{x_{d_{1}}}\right) \times 1 \\
1
\end{array}\right], E_{4}=\left[\begin{array}{c}
E_{3} \\
0
\end{array}\right]
$$

and

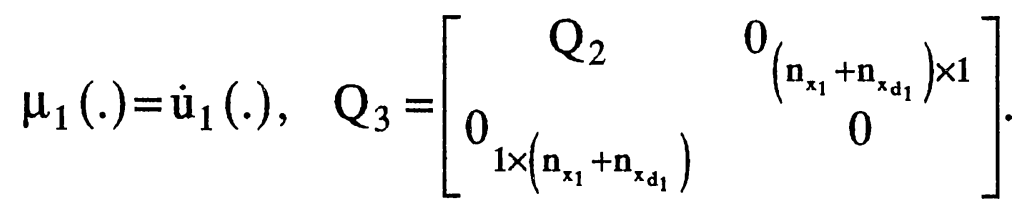

For the system (5.12), the following equations solve for the optimal controller gain matrix, $F_{1}^{*}$, minimizing the index of performance $\mathrm{J}\left(\mathrm{u}_{1}\right)$ given in Equation (5.13).

$$
\begin{aligned}
& A_{4}^{\prime} P_{1}+P_{1} A_{4}+Q_{3}-\frac{1}{g_{1}} P_{1} B_{4} B_{4}^{\prime} P_{1}=0_{n_{x_{4}} \times n_{x_{4}}} \\
& F_{1}=\frac{1}{g_{1}} B_{4}^{\prime} P_{1}=\frac{1}{g_{1}}\left[\begin{array}{llll}
p_{n_{x_{4}} 1} & p_{n_{x_{4}}} & \cdot & p_{n_{x_{4}} n_{x_{4}}}
\end{array}\right] \\
& =\left[\begin{array}{llll}
f_{1} & f_{2} & \cdot & f_{n_{x_{4}}}
\end{array}\right] \\
& F_{1}^{*}=\left[\begin{array}{llll}
f_{1}^{*} & f_{2}^{*} & \cdot & f_{n_{x_{4}-1}}^{*}
\end{array}\right], f_{i}^{*}=\tau_{n_{1}} \times f_{i} \quad i=1,2, \ldots, n_{x_{4}}-1
\end{aligned}
$$

where

$$
\tau_{n_{1}}=\frac{1}{f_{n_{x_{4}}}} \text {, motor lag delay for lead pilot }
$$

and $\mathrm{n}_{\mathrm{x}_{4}}=\mathrm{n}_{\mathrm{x}_{1}}+\mathrm{n}_{\mathrm{x}_{\mathrm{d}_{1}}}+1$.

To determine the optimal estimate of the augmented state $\underline{x}_{4}$, we use the following Kalman filter. 


$$
\underline{\hat{\hat{x}}}_{4}(\mathrm{t})=\mathrm{A}_{5} \underline{\hat{x}}_{4}(\mathrm{t})+\mathrm{K}_{1} \mathrm{C}_{4}\left[\underline{\mathrm{x}}_{4}(\mathrm{t})-\underline{\hat{x}}_{4}(\mathrm{t})\right]+\underline{\mathrm{b}}_{5} \mathrm{u}_{\mathrm{c}_{1}}(\mathrm{t})+\mathrm{K}_{1} \underline{\mathrm{v}}_{\mathrm{y}_{1}}
$$

where

$$
\left.A_{5}=\left[\begin{array}{cc}
A_{3} & B_{3} \\
0 & -\frac{1}{\tau_{n_{1}}}
\end{array}\right], \underline{b}_{5}+n_{x_{1}}\right)=\left[\begin{array}{c}
0\left(n_{x_{1}}+n_{x_{1}}\right) \times 1 \\
\frac{1}{\tau_{n_{1}}}
\end{array}\right], C_{4}=\left[\begin{array}{ll}
C_{3} & \underline{d_{3}}
\end{array}\right]
$$

The augmented state $\underline{x}_{4}$ satisfies the dynamical equation

$$
\underline{\dot{x}}_{4}(t)=A_{5} \underline{x}_{4}(t)+\underline{b}_{5} u_{c_{1}}(t)+E_{5} \underline{w}_{1}(t)
$$

where

$$
\underline{w}_{1}(.)=\left[\begin{array}{c}
\underline{w}(.) \\
v_{u}(.)
\end{array}\right], E_{5}=\left[\begin{array}{cc}
E_{3} & 0 \\
0 & \frac{1}{\left.n_{x_{1}}+n_{x_{d_{1}}}\right) \times 1} \\
0_{1 \times n_{w}} & \frac{\tau_{n_{1}}}{n}
\end{array}\right]
$$

and

$$
\mathrm{u}_{\mathrm{c}_{1}}(\mathrm{t})=-\left[\begin{array}{ll}
\mathrm{F}_{1}^{*} & 0
\end{array}\right] \underline{\hat{\mathrm{x}}}_{4}(\mathrm{t}) \text {. }
$$

The estimator gain, $K_{1}$ is determined by

$$
\mathrm{K}_{1}=\mathrm{S}_{1} \mathrm{C}_{4}^{\prime} \mathrm{V}_{\mathrm{y}}^{-1}
$$

where, the positive definite symmetric matrix $S_{1}$ is the solution of the Riccati equation

$$
A_{5} S_{1}+S_{1} A_{5}^{\prime}+W_{1}-S_{1} C_{4}^{\prime} V_{y}^{-1} C_{4} S_{1}=0_{n_{x_{4}} \times n_{x_{4}}}
$$

where

$$
\mathrm{W}_{1}=\operatorname{Cov}\left(\mathrm{E}_{5} \underline{\mathrm{w}}_{1}\right)=\mathrm{E}_{5}\left[\begin{array}{cc}
\mathrm{W} & 0_{\mathrm{n}_{\mathrm{w}} \times 1} \\
0_{1 \times \mathrm{n}_{\mathrm{w}}} & \mathrm{V}_{\mathrm{u}_{1}}
\end{array}\right] \mathrm{E}_{5}^{\prime}=\left[\begin{array}{cc}
\mathrm{E}_{3} \mathrm{WE}_{3}^{\prime} & 0_{\left(\mathrm{n}_{\mathrm{x}_{1}}+\mathrm{n}_{\mathrm{x}_{\mathrm{d}} 1}\right) \times 1} \\
0_{1 \times\left(\mathrm{n}_{\mathrm{x}_{1}}+\mathrm{n}_{\left.\mathrm{x}_{\mathrm{d} 1}\right)}\right)} & \frac{\mathrm{V}_{\mathrm{u}_{1}}}{\tau_{\mathrm{n}_{1}}^{2}}
\end{array}\right]
$$


and

$$
E\left\{v_{u_{1}}(t) v_{u_{1}}(\tau)\right\}=V_{u_{1}} \delta(t-\tau)
$$

To find the overall closed-loop system containing the first OCM (lead pilot), we may proceed as follows. By using Equation (5.20) in the Equations (5.18) and (5.19), we will have the following equations.

$$
\begin{aligned}
\underline{\dot{x}}_{4}(\mathrm{t})= & \mathrm{A}_{5} \underline{\mathrm{x}}_{4}(\mathrm{t})-\underline{\mathrm{b}}_{5} \mathrm{~F}_{1}^{*} \underline{\hat{\mathrm{x}}}_{4}(\mathrm{t})+\mathrm{E}_{5} \underline{\mathrm{w}}_{1}(\mathrm{t}) \\
\underline{\hat{\mathrm{x}}}_{4}(\mathrm{t})= & \mathrm{A}_{5} \underline{\hat{\mathrm{x}}}_{4}(\mathrm{t})+\mathrm{K}_{1} \mathrm{C}_{4}\left[\underline{\mathrm{x}}_{4}(\mathrm{t})-\underline{\hat{x}}_{4}(\mathrm{t})\right] \\
& -\underline{\mathrm{b}}_{5} \underline{\hat{\mathrm{x}}}_{4}(\mathrm{t})+\mathrm{K}_{1} \underline{\mathrm{v}}_{\mathrm{y}_{1}}(\mathrm{t}) .
\end{aligned}
$$

Now, we define the new augmented state $\underline{x}_{5}($.$) and new external$ input vector $\underline{\mathrm{w}}_{2}($.$) as$

$$
\underline{\mathrm{x}}_{5}(.)=\left[\begin{array}{l}
\underline{\mathrm{x}}_{4}(.) \\
\underline{\hat{x}}_{4}(.)
\end{array}\right], \quad \underline{\mathrm{w}}_{2}(.)=\left[\begin{array}{c}
\underline{\mathrm{w}}_{1}(.) \\
\underline{\mathrm{v}}_{1}(.)
\end{array}\right]
$$

where

$$
\begin{aligned}
& \underline{\mathrm{x}}_{5} \text {-is a } 2 \times \mathrm{n}_{\mathrm{x}_{4}} \text {-dimensional column vector, } \\
& \quad \mathrm{n}_{\mathrm{x}_{4}}=\mathrm{n}_{\mathrm{x}_{1}}+\mathrm{n}_{\mathrm{x}_{\mathrm{d} 1}}+1 \\
& \underline{\mathrm{w}}_{2} \text {-is an } \mathrm{n}_{\mathrm{w}_{2}} \text {-dimensional column vector, } \mathrm{n}_{\mathrm{w}_{2}}=\mathrm{n}_{\mathrm{w}}+1+\mathrm{n}_{\mathrm{v}_{\mathrm{y} 1}} .
\end{aligned}
$$

In terms of $\underline{x}_{5}($.$) , the combined Equations (5.23) and (5.24) becomes$

$$
\underline{\dot{x}}_{5}(t)=A_{6} \underline{x}_{5}(t)+E_{6} \underline{w}_{2}(t)
$$

where

$$
\mathrm{A}_{6}=\left[\begin{array}{cc}
\mathrm{A}_{5} & -\underline{\mathrm{b}}_{5} \mathrm{~F}_{1}^{*} \\
\mathrm{~K}_{1} & \mathrm{~A}_{5}-\underline{\mathrm{b}}_{5} \mathrm{~F}_{1}^{*}-\mathrm{K}_{1} \mathrm{C}_{4}
\end{array}\right],
$$


and $E_{6}=\left[\begin{array}{cc}E_{5} & 0_{n_{x_{4}} \times n_{v_{y 1}}} \\ 0_{n_{x_{4}} \times\left(n_{w}+1\right)} & K_{1}\end{array}\right]$.

The output of the vehicle 1 , in terms of $\underline{x}_{5}(t)$ becomes

$$
\underline{y}_{1}(t)=C_{5} \underline{x}_{5}(t)
$$

where

$$
\mathrm{C}_{5}=\left[\begin{array}{ll}
\mathrm{C}_{4} & 0_{\mathrm{n}_{\mathrm{y}_{1}} \times \mathrm{n}_{\mathrm{x} 4}}
\end{array}\right] .
$$

The Equations of (5.26) and (5.27) describe the closed loop dynamics of vehicle 1 (see Figure 5.1).

\subsubsection{OCM2 Development}

The second pilot (Wingman) observes the delayed error (and error rate). In order to determine the delayed errors, we may proceed as follows. We assume that the following equations model the intrasimulator time delay $\tau_{3}$.

$$
\begin{aligned}
& \underline{\dot{x}}_{d_{3}}=A_{d_{3}} \underline{x}_{d_{3}}+\underline{b}_{d_{3}} u_{\tau_{3}} \\
& y_{d_{3}}=C_{d_{3}} \underline{x}_{d_{3}}+d_{d_{3}} u_{\tau_{3}}
\end{aligned}
$$

where

$\underline{x}_{d_{3}}-$ an $n_{x_{d_{3}}}$-dimensional state vector

$\mathbf{u}_{\tau_{3}}$ - a scalar input

$\mathrm{y}_{\mathrm{d}_{3}}$ - a scalar output

$\mathrm{A}_{\mathrm{d}_{3}}$-an $\mathrm{n}_{\mathrm{x}_{\mathrm{d} 3}} \times \mathrm{n}_{\mathrm{x}_{\mathrm{d} 3}}$-dimensional constant matrix

$\underline{b}_{d_{3}}$-an $n_{x_{d 3}}$-dimensional constant column vector

$\mathrm{C}_{\mathrm{d}_{3}}$-an $\mathrm{n}_{\mathrm{x}_{\mathrm{d} 3}}$-dimensional constant row vector

$d_{d_{3}}-a$ scalar 
Becuase we have $\mathrm{n}_{\mathrm{v}_{\mathrm{y} 1}}$ displayed variables, $\underline{\mathrm{y}}_{1}$, each passes through delay $\tau_{3}$, the following augmented state equations relate the output vector $\underline{y}_{1}$, as an input to the delay element $\tau_{3}$, to the delayed output $\underline{y}_{1}^{d}$ which is the output of the time delay $\tau_{3}$ (see Figure 5.2).

$$
\begin{aligned}
& \underline{\dot{x}}_{D_{3}}(t)=A_{D_{3}} \underline{x}_{D_{3}}(t)+B_{D_{3}} C_{5} \underline{x}_{5} \\
& \underline{y}_{1}^{d}(t)=C_{D_{3}} \underline{x}_{D_{3}}(t)+D_{D_{3}} C_{5} \underline{x}_{5}
\end{aligned}
$$

where

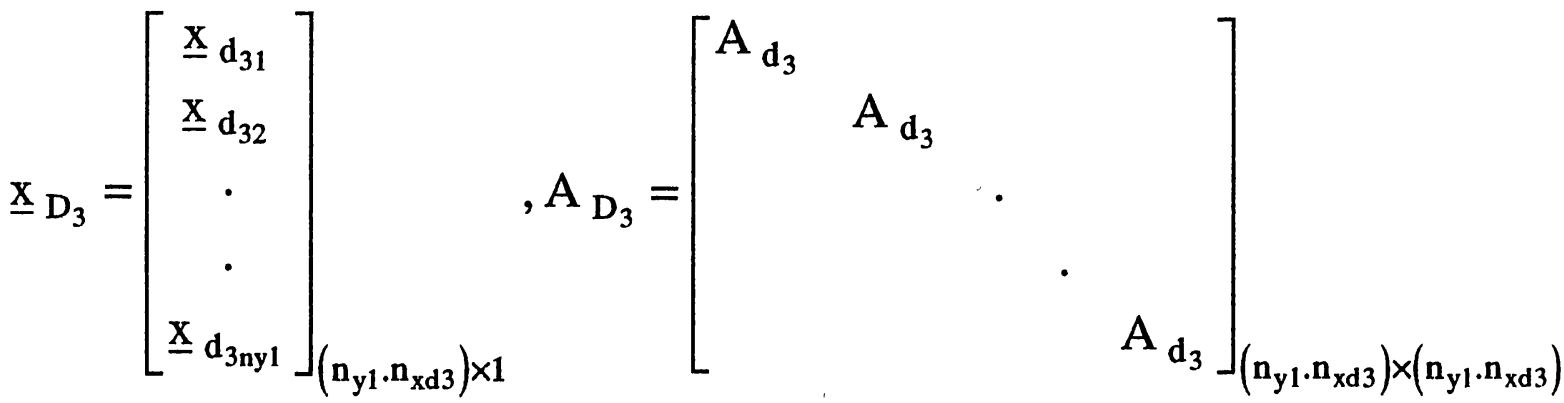

$$
\begin{aligned}
& B_{D_{3}}=\left[\begin{array}{llll}
\underline{b}_{d_{3}} & & & \\
& \underline{b}_{d_{3}} & & \\
& & \cdot & \\
& & & \\
& & & \underline{b}_{d_{3}}
\end{array}\right]_{\left(n_{y 1} \cdot n_{\mathrm{xd} 3}\right) \times n_{y 1}}
\end{aligned}
$$
and
$\mathrm{C}_{\mathrm{D}_{3}}=\left[\begin{array}{llll}\mathrm{C}_{\mathrm{d}_{3}} & & & \\ & \cdot & & \\ & & \cdot & \\ & & \mathrm{C}_{\mathrm{d}_{3}}\end{array}\right]_{\left(\mathrm{n}_{\mathrm{y} 1}\right) \times\left(\mathrm{n}_{\mathrm{y} 1} \cdot \mathrm{n}_{\mathrm{xd} 3}\right)}, \mathrm{D}_{\mathrm{D}_{3}}=\left[\begin{array}{lll}\mathrm{d}_{\mathrm{d}_{3}} & & \\ & \cdot & \\ & \cdot & \\ & & \mathrm{d}_{\mathrm{d} 3}\end{array}\right]_{\mathrm{n}_{\mathrm{y} 1} \times \mathrm{n}_{\mathrm{y} 1}}$. 


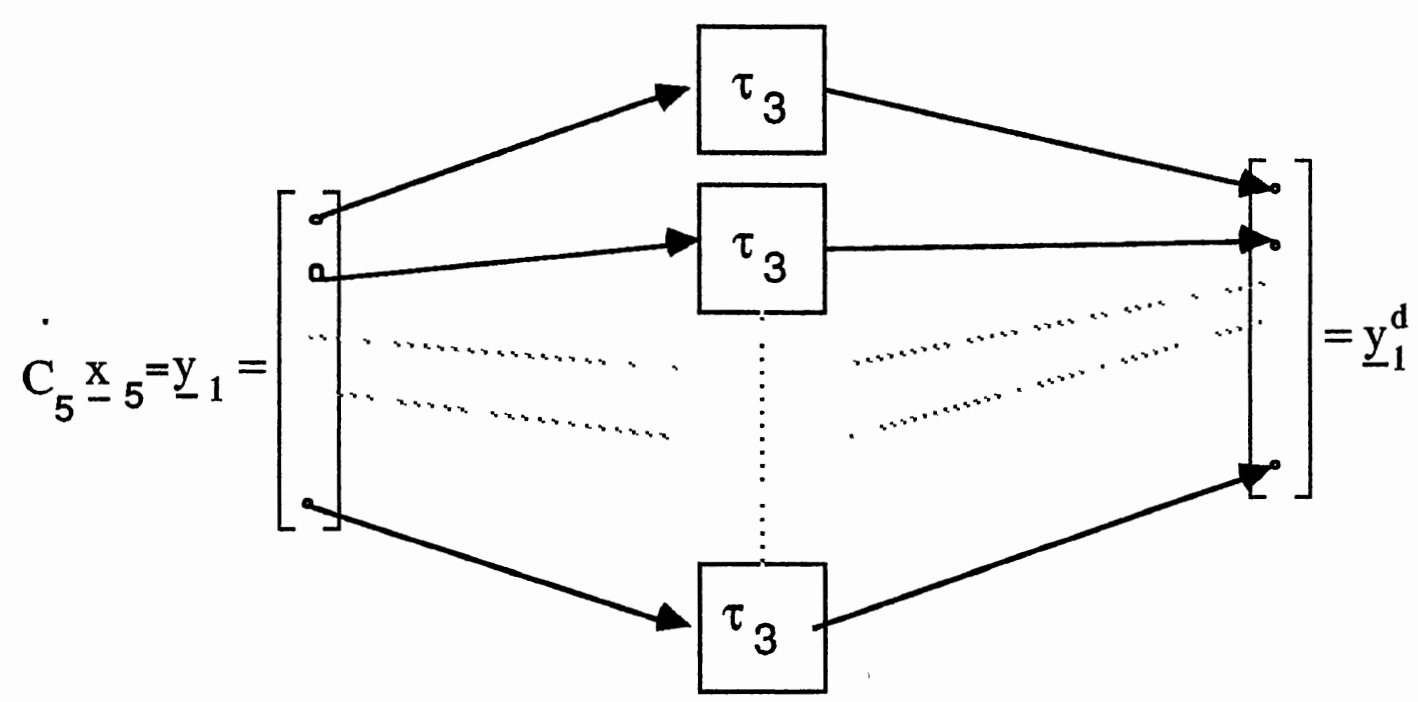

Figure 5.2. Input/Output Relationship For Model (5.29)

Let us combine the closed-loop system $\underline{x}_{5}$ and the delayed states $\underline{\mathrm{x}}_{\mathrm{D}_{3}}$ in an augmented state $\underline{\mathrm{x}}_{6}$.

$$
\underline{\mathrm{x}}_{6}(.)=\left[\begin{array}{c}
\underline{\mathrm{x}}_{5}(.) \\
\underline{\mathrm{x}}_{\mathrm{D}_{3}}(.)
\end{array}\right]_{\mathrm{n}_{\mathrm{x} 6} \times 1}, \mathrm{n}_{\mathrm{x}_{6}}=2\left(\mathrm{n}_{\mathrm{x}_{1}}+\mathrm{n}_{\mathrm{x}_{\mathrm{d} 1}}+1\right)+\mathrm{n}_{\mathrm{y}_{1}} \cdot \mathrm{n}_{\mathrm{x}_{\mathrm{d} 3}} .
$$

In terms of $\underline{x}_{6}($.$) , we may have the following augmented system$

$$
\begin{aligned}
& \underline{\dot{x}}_{6}(t)=A_{7} \underline{x}_{6}+E_{7} \underline{w}_{2}(t) \\
& \underline{y}_{1}^{d}(t)=C_{6} \underline{x}_{6}
\end{aligned}
$$

where

$$
A_{7}=\left[\begin{array}{cc}
A_{6} & 0_{2 n_{x 4} \times n_{y 1} \cdot n_{x_{d} 3}} \\
B_{D_{3}} C_{5} & A_{D_{3}}
\end{array}\right], E_{7}=\left[\begin{array}{c}
E_{6} \\
0_{n_{y 1} \cdot n_{x_{d} 3} \times 1}
\end{array}\right]
$$

and 
$\mathrm{C}_{6}=\left[\begin{array}{lll}\mathrm{D}_{\mathrm{D}_{3}} \mathrm{C}_{5} & \mathrm{C}_{\mathrm{D}_{3}}\end{array}\right]$

Now, we have a special tracking problem illustrated in Figures $5.3 \& 5.4$, where the state disturbance noise of the reference model and that of the plant model are correlated.

As seen in Figure 5.4, $\mathrm{n}_{\mathrm{x}_{6}}$ states of the overall closed-loop system are uncontrollable from $u_{2}$. These uncontrollable states are asymptotically stable because the matrix $A_{7}$ is asymptotically stable (see Appendix B). Therefore, we may develop an optimal controller for this tracking problem as follows.

Let us assume that the operation of the second vehicle is defined by the equations (see Figure 5.4)

$$
\begin{aligned}
& \underline{\dot{x}}_{2}(t)=A_{2} \underline{x}_{2}(t)+\underline{b}_{6} u_{d_{2}}(t)+E_{2} \underline{w}(t) \\
& \underline{y}_{2}(t)=C_{2} \underline{x}_{2}(t)+\underline{d}_{2} u_{d_{2}}(t)
\end{aligned}
$$

where

$\underline{x}_{2}-$ an $n_{x_{2}}-$ dimensional state vector

$u_{d_{2}}$-a scalar input to the vehicle 2

$\underline{\mathrm{y}}_{2}$-an $\mathrm{n}_{\mathrm{y}_{1}}$ - dimensional output vector

$A_{2}-n_{x_{2}} \times n_{x_{2}}$-dimensional constant matrix

$\underline{\mathrm{b}}_{2}-\mathbf{n}_{\mathrm{x}_{2}}-$ dimensional column vector

$\mathrm{E}_{2}-\mathrm{n}_{\mathrm{x}_{2}} \times \mathrm{n}_{\mathrm{w}}$-dimensional constant matrix

$\mathrm{C}_{2}-\mathrm{n}_{\mathrm{y}_{1}} \times \mathrm{n}_{\mathrm{x}_{1}}$-dimensional constant matrix

$\underline{\mathrm{d}}_{2}-\mathrm{n}_{\mathrm{y}_{1}}$-dimensional constant column vector 


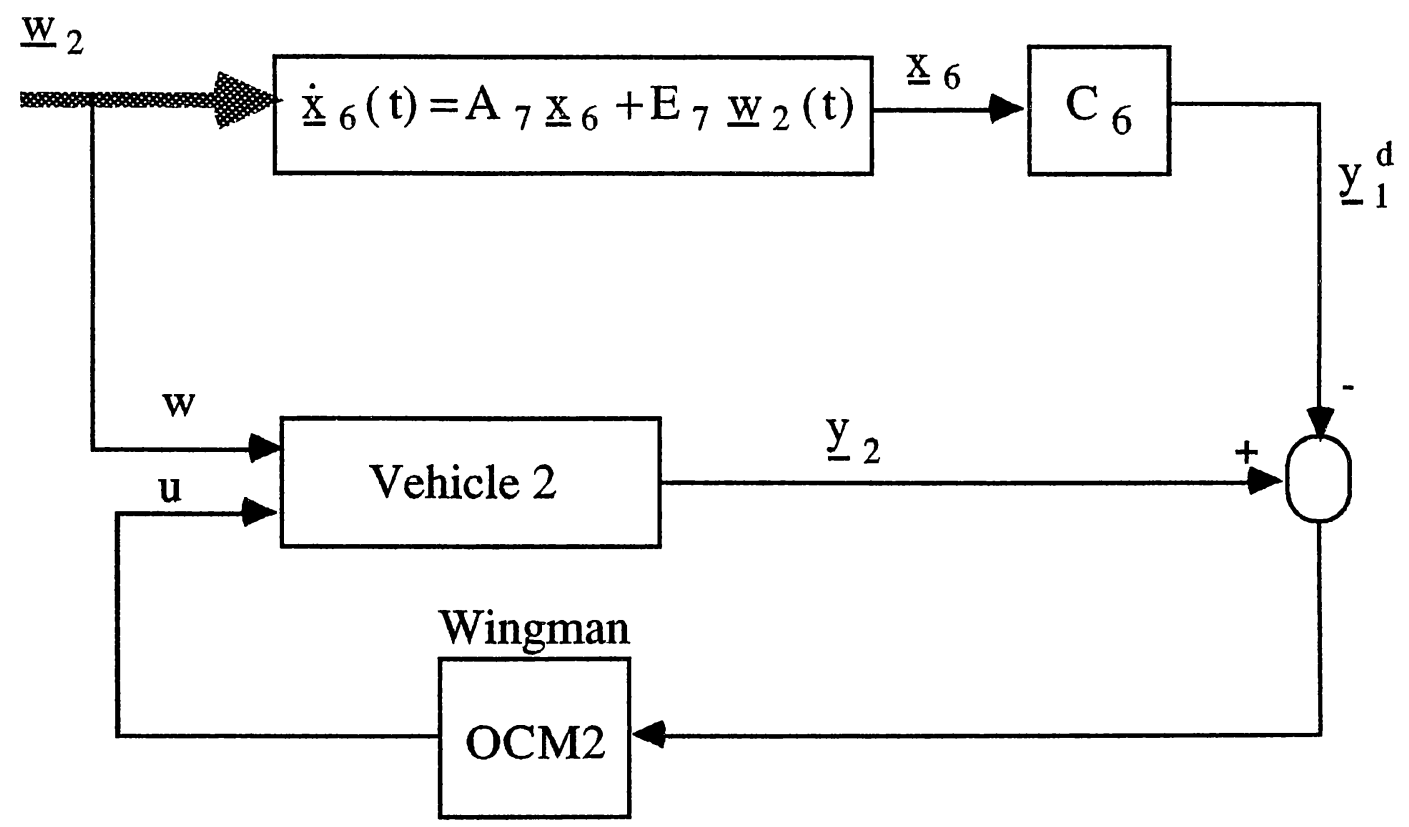

Figure 5.3. Tracking Configuration Of The Dual OCM

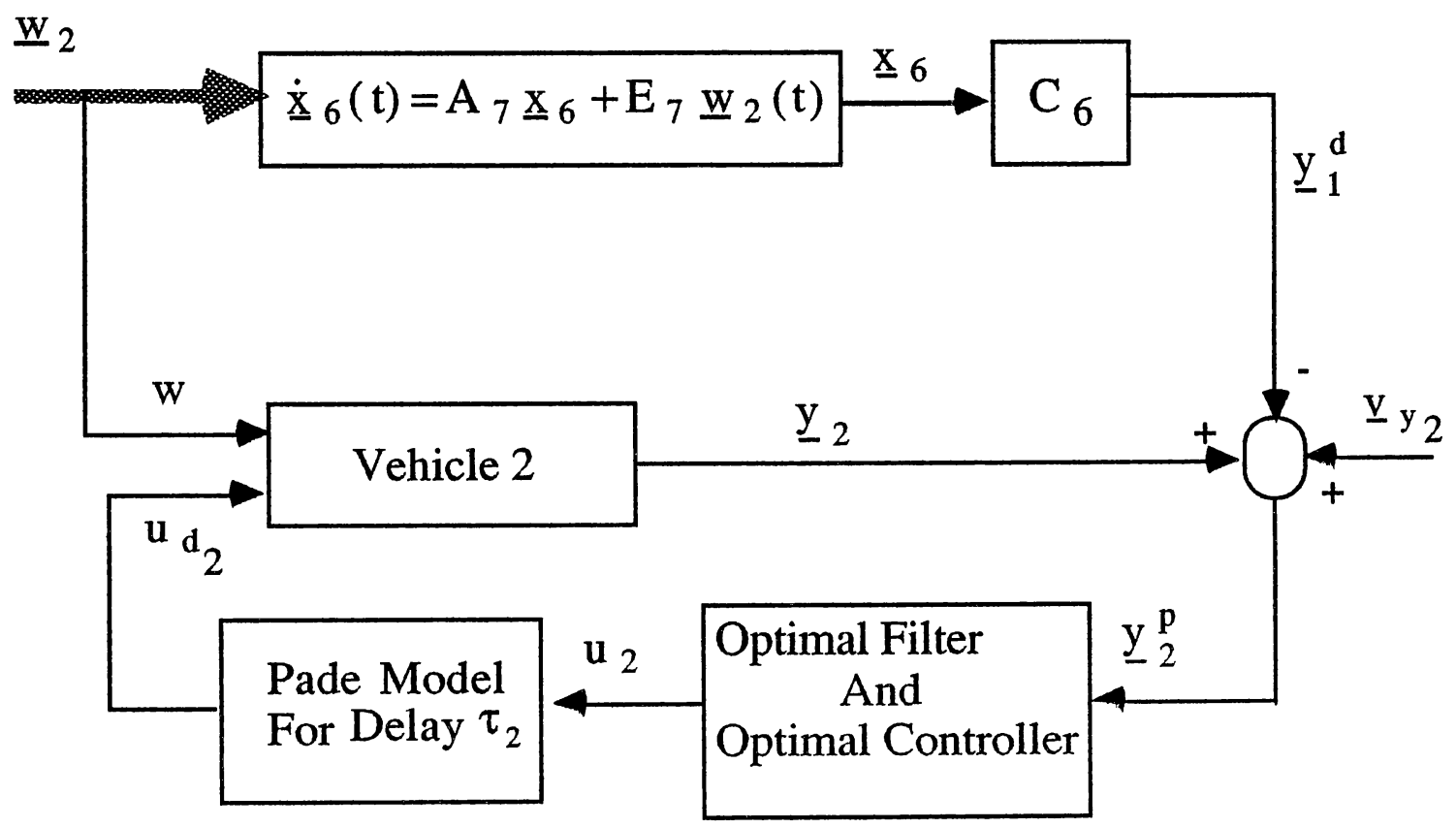

Figure 5.4. Using Modified OCM In Figure 5.3 
Now, we combine the reference model (5.30) and plant model (5.31) in an augmented system in order to develop the second OCM. In terms of the augmented state $\underline{x}_{7}()=.\left[\begin{array}{l}\underline{x}_{6}(.) \\ \underline{x}_{2}(.)\end{array}\right]$, we write

$$
\underline{\dot{x}}_{7}(t)=A_{8} \underline{x}_{7}(t)+\underline{b}_{8} u_{d_{2}}(t)+E_{8} \underline{w}_{2}(t)
$$

where

$$
A_{8}=\left[\begin{array}{cc}
A_{7} & 0_{n_{x_{6}} \times n_{x_{6}}} \\
0 n_{n_{2} \times n_{x_{6}}} & A_{2}
\end{array}\right], \underline{b}_{8}=\left[\begin{array}{c}
0 n_{x_{6} \times 1} \\
\underline{b}_{2}
\end{array}\right], E_{8}=\left[\begin{array}{c}
E_{7} \\
E_{2} \cdot T_{e}
\end{array}\right]
$$

with

$$
\mathrm{T}_{\mathrm{e}}=\left[\begin{array}{ccccc}
1 & 0 & \cdot & \cdot & 0 \\
1 & 0 & \cdot & \cdot & 0 \\
\cdot & \cdot & \cdot & \cdot & \cdot \\
\cdot & \cdot & \cdot & \cdot & \cdot \\
1 & 0 & . & \cdot & 0
\end{array}\right]_{\mathrm{n}_{\mathrm{w}} \times\left(\mathrm{n}_{\mathrm{y}_{1}}+2\right)}
$$

The output variables for the augmented system are

$$
\left[\begin{array}{c}
\underline{y}_{1}^{d} \\
\underline{y}_{2}
\end{array}\right]=\left[\begin{array}{cc}
C_{6} & 0_{n_{y_{1}} \times n_{x_{2}}} \\
0_{n_{x_{2}} \times n_{x_{6}}} & C_{2}
\end{array}\right] \underline{x}_{7}+\left[\begin{array}{c}
0_{n_{y_{1}} \times 1} \\
\underline{d}_{2}
\end{array}\right] u_{d_{2}}
$$

In order to change the above tracking problem to a standard OCM problem, we need to change the output variables (5.33) as

$$
E_{r}(t)=\underline{y}_{2}(t)-\underline{y}_{1}^{d}(t)=\left[\begin{array}{ll}
C_{2} & -C_{6}
\end{array}\right] \underline{x}_{7}(t)+\underline{d}_{2} u_{d_{2}}(t)
$$




$$
\begin{aligned}
\underline{y}_{2}^{p}(t) & =E_{r}(t)+\underline{v}_{y_{2}}(t)= \\
& =\underbrace{\left[\begin{array}{ll}
C_{2}-C_{6}
\end{array}\right] \underline{x}_{7}(t)+\underline{d}_{2} u_{d_{2}}(t)+\underline{v}_{y_{2}}(t) .}_{C_{8}}
\end{aligned}
$$

The displayed variables given in (5.35) are perceived by the human operator in the second OCM (Wingman).

Now, As illustrated in Figure 5.5, we wish to develop a modified OCM for the controlled system described by Equation (5.32) with displayed dynamics given in (5.35). Let us assume the following Pade approximant for delay $\tau_{2}$.

$$
\begin{aligned}
& \underline{\dot{x}}_{d_{2}}(t)=A_{d_{2}} \underline{x}_{d_{2}}(t)+\underline{b}_{d_{2}} u_{2}(t) \\
& u_{d_{2}}(t)=C_{d_{2}} \underline{x}_{d_{2}}(t)+d_{d_{2}} u_{2}(t)
\end{aligned}
$$

where

$$
\begin{aligned}
& \underline{x}_{d_{2}}-\text { an } n_{x_{2}} \text {-dimensional state vector } \\
& u_{2}-\text { a scalar input } \\
& u_{d_{2}}-a \text { scalar output } \\
& A_{d_{2}} \text {-an } n_{x_{d 2}} \times n_{x_{d 2}} \text {-dimensional constant matrix } \\
& \underline{b}_{d_{2}} \text {-an } n_{x_{2}}-\text { dimensional constant column vector } \\
& C_{d_{2}} \text {-an } n_{x_{2}} \text {-dimensional constant row vector } \\
& d_{d_{2}}-a \text { scalar. }
\end{aligned}
$$

Further, it is assumed that the Wingman is attempting to minimize the following cost function.

$$
\mathrm{J}\left(\mathrm{u}_{2}\right)=\mathrm{E}\left\{\mathrm{e}^{2}(\mathrm{t})+\mathrm{g}_{2} \dot{\mathrm{u}}_{2}^{2}(\mathrm{t})\right\}
$$


Where $e(t)$ denotes the error function which can be defined as an actual error (an element of vector quantity $\underline{y}_{2}(t)-\underline{y}_{1}(t)$ ) or a delayed error (an element of $\left.\underline{y}_{2}(t)-\underline{y}_{1}^{d}\right)$. Hence, the error function, e(t), can be defined appropriately as a function of components of the augmented state $\underline{x}_{7}(t)$, and Equation (5.37) can be rewritten as

$$
\mathrm{J}\left(\mathrm{u}_{2}\right)=\mathrm{E}\left[\underline{\mathrm{x}}_{7}^{\prime}(\mathrm{t}) \mathrm{Q}_{7} \underline{\mathrm{x}}_{7}(\mathrm{t})+\mathrm{g} \dot{\mathrm{u}}_{2}^{2}(\mathrm{t})\right]
$$

where $\mathrm{Q}_{7}$ is an appropriate compatible nonnegative definite symmetric constant matrix.

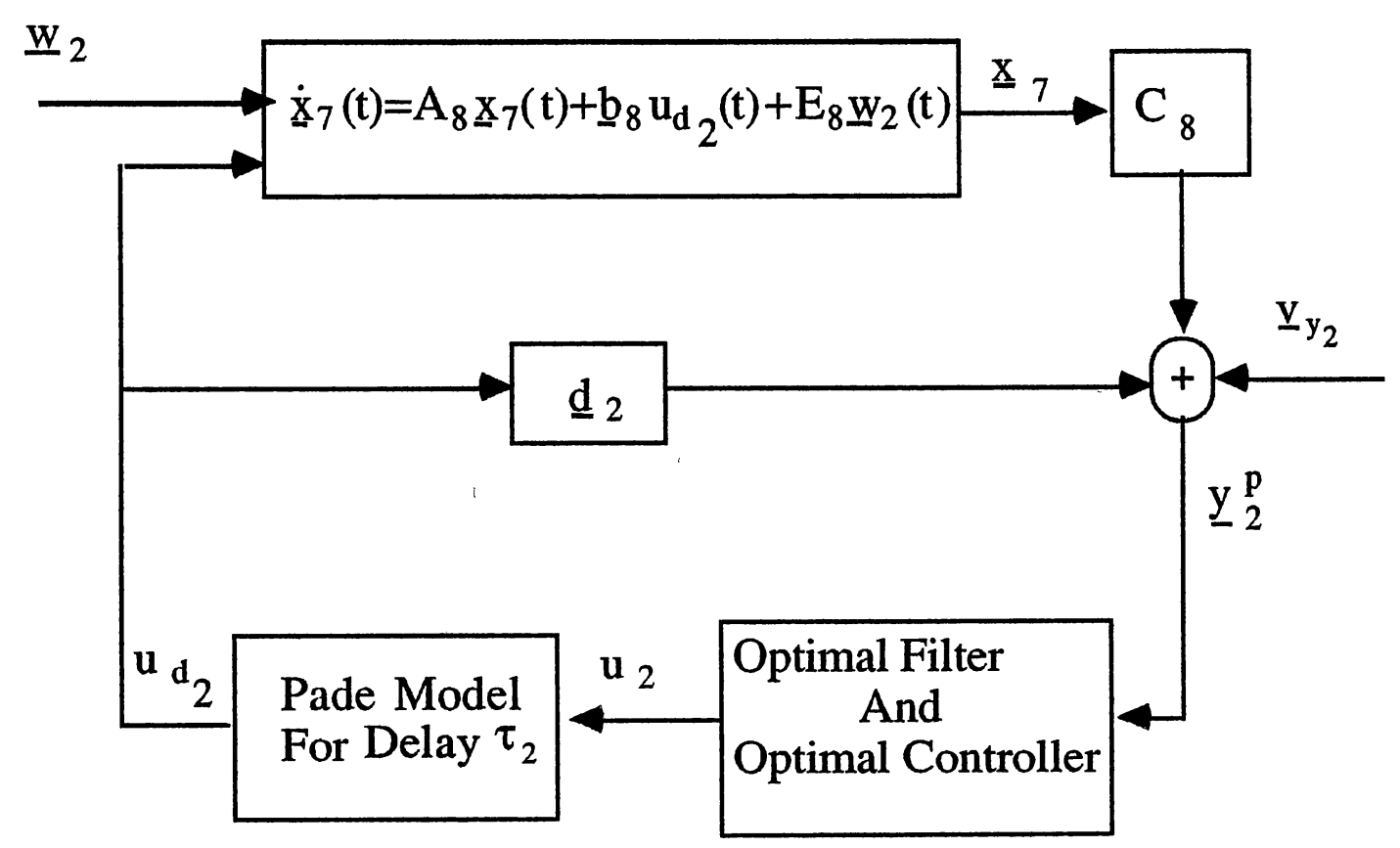

Figure 5.5 Block-Diagram Of Modified OCM For Wingman 
The external inputs $\underline{w}_{2}(t), \underline{v}_{y_{2}}(t)$ and $v_{u}(t)$ are assumed to be independent white Gaussian noises with

$$
\begin{aligned}
& E\left\{\underline{w}_{2}(t) \underline{w}_{2}^{\prime}(\tau)\right\}=W_{2} \delta(t-\tau), W_{2}=\left[\begin{array}{ccc}
W & 0_{n_{w} \times 1} & 0_{n_{w} \times n_{y_{1}}} \\
0_{1 \times n_{w}} & v_{u} & 0_{1 \times n_{y_{1}}} \\
0_{n_{y_{1}} \times n_{w}} & 0_{n_{y_{1}} \times 1} & v_{y_{1}}
\end{array}\right] \\
& E\left\{v_{u_{2}}(t) v_{u_{2}}(\tau)\right\}=V_{u_{2}} \delta(t-\tau) \\
& E\left\{\underline{v}_{y_{2}}(t) \underline{v}_{y_{2}}^{\prime}(\tau)\right\}=V_{y_{2}} \delta(t-\tau)
\end{aligned}
$$

where

$$
\mathrm{v}_{\mathrm{y}_{2}}=\left[\begin{array}{ccccc}
\mathrm{v}_{\mathrm{y}_{2,1}} & 0 & \cdot & \cdot & 0 \\
\cdot & \mathrm{v}_{\mathrm{y}_{2,2}} & 0 & \cdot & 0 \\
\cdot & 0 & \cdot & \cdot & \cdot \\
0 & \cdot & \cdot & \cdot & 0 \\
0 & \cdot & \cdot & 0 & \mathrm{v}_{\mathrm{y}_{2, \mathrm{n}_{1} 1}}
\end{array}\right]_{\left(\mathrm{n}_{\mathrm{y}_{1}} \times \mathrm{n}_{\mathrm{y}_{1}}\right)}
$$

and

$$
\mathrm{E}\left\{\underline{\mathrm{v}}_{\mathrm{y}_{2_{\mathrm{i}}}}(\mathrm{t}) \underline{\mathrm{v}}_{\mathrm{y}_{2_{\mathrm{j}}}}^{\prime}(\tau)\right\}=\left\{\begin{array}{cccc}
\mathrm{v}_{\mathrm{y}_{2, i}} \delta(\mathrm{t}-\tau) & \text { if } & \mathrm{i}=\mathrm{j} \\
0 & \text { if } & \mathrm{i} \neq \mathrm{j}
\end{array}\right\},
$$

$\underline{v}_{y_{i}}$ denotes the $i$-th component of the vector $\underline{v}_{y_{2}}$.

Now, in order to develop the Wingman's OCM, we may proceed as just described for the first OCM. The Equations (5.1) through (5.27) remain the same except that $A_{1}, \underline{b}_{1}, E_{1}, C_{1}, \underline{d}_{1}, A_{d_{1}}, \underline{b}_{d_{1}}, C_{d_{1}}, \underline{d}_{d_{1}}, Q_{1}$ and $\underline{w}(t)$ are respectively replaced by $A_{8}, \underline{b}_{8}, E_{8}, C_{8}, \underline{d}_{2}, A_{d_{2}}, \underline{b}_{d_{2}}, C_{d_{2}}$, 
$\underline{\mathrm{d}}_{\mathrm{d}_{2}}, \mathrm{Q}_{7}$ and $\underline{w}_{2}(\mathrm{t})$. Below, we summarize the main results.

$$
\begin{array}{r}
\underline{\dot{x}}_{T}(t)=A_{T} \underline{x}_{T}(t)-\underline{b}_{T} F_{2}^{*} \underline{\hat{x}}_{T}(t)+E_{T} \underline{w}_{T}(t) \\
\underline{\hat{x}}_{T}(t)=A_{T} \underline{\hat{x}}_{T}(t)+K_{2} C_{T}\left[\underline{x}_{T}(t)-\underline{\hat{x}}_{T}(t)\right] \\
-\underline{b}_{T} \underline{\hat{x}}_{T}(t)+K_{2} \underline{y}_{y_{2}}(t)
\end{array}
$$

where

$$
\begin{aligned}
& \underline{x}_{T}(t)=\left[\begin{array}{c}
\underline{x}_{d_{2}}(t) \\
\underline{x}_{7}(t) \\
u_{2}(t)
\end{array}\right], A_{T}=\left[\begin{array}{ccc}
A_{d_{2}} & 0\left(n_{\left.x_{2} \times n_{x_{7}}\right)}\right. & \underline{b}_{d_{2}} \\
\underline{b}_{8} C_{d_{2}} & A_{8} & \underline{b}_{8} d_{d_{2}} \\
0_{1 \times n_{x_{d} 2}} & 0_{1 \times n_{x_{7}}} & -\frac{1}{\tau_{n_{2}}}
\end{array}\right], \underline{b}_{T}=\left[\begin{array}{c}
0_{n_{x_{d}} \times 1} \\
0_{n_{x_{7} \times 1}} \\
\frac{1}{\tau_{n_{2}}}
\end{array}\right] \\
& \underline{\mathrm{w}}_{\mathrm{T}}(\mathrm{t})=\left[\begin{array}{cc}
\underline{\mathrm{w}}_{2}(\mathrm{t}) \\
\mathrm{v}_{\mathrm{u}_{2}}(\mathrm{t})
\end{array}\right], \mathrm{E}_{\mathrm{T}}=\left[\begin{array}{cc}
0_{1 \times\left(\mathrm{n}_{\mathrm{w}}+1+\mathrm{n}_{\mathrm{y}_{1}}\right)} & 0_{\mathrm{n}_{\mathrm{x}_{\mathrm{d}_{2}}} \times 1} \\
\mathrm{E}_{8} & 0_{\mathrm{n}_{\mathrm{x}_{7} \times 1}} \\
0_{1 \times\left(\mathrm{n}_{\mathrm{w}}+1+\mathrm{n}_{\mathrm{y}_{1}}\right)} & \frac{1}{\tau_{\mathrm{n}_{2}}}
\end{array}\right]
\end{aligned}
$$

and

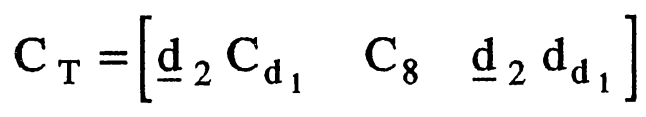

with

$$
\mathrm{n}_{\mathrm{x}_{7}}=2\left(\mathrm{n}_{\mathrm{x}_{1}}+\mathrm{n}_{\mathrm{x}_{\mathrm{d}_{1}}}+1\right)+\mathrm{n}_{\mathrm{y}_{1}} \cdot \mathrm{n}_{\mathrm{x}_{7}}+\mathrm{n}_{\mathrm{x}_{2}}
$$

$\underline{\mathrm{x}}_{\mathrm{T}}$ is the closed-loop state, of order $\mathbf{n}_{\mathrm{x}_{\mathrm{T}}}=\mathrm{n}_{\mathrm{x}_{7}}+\mathbf{n}_{\mathrm{x}_{\mathrm{d}_{2}}}+1$.

Equations (5.39) describe the overall closed-loop dual OCM system. The optimal controller gain factor, $\mathrm{F}_{2}{ }^{*}$, minimizes the performance index $\mathrm{J}\left(\mathrm{u}_{2}\right)$ given in Equation (5.38) and $\mathrm{K}_{2}$ denotes the 
estimator gain matrix. The matrices $\mathrm{F}_{2}{ }^{*}, \mathrm{~K}_{2}$ and the motor lag for the Wingman, $\tau_{\mathrm{n}_{2}}$, are respectively determined by Equations (5.14), (5.15), (5.17) and (5.21), with new matrix replacements mentioned above. The overall system performance (closed loop transfer function), the mean square error and the mean square control input can be determined just as discussed in chapter IV.

\subsection{Summary}

In this chapter we considerd and solved the dual OCM problem.in general for the case in which the first OCM does not need to interact with the second OCM. The solution of this problem may be best summarized in Figure 5.6. Here, we assumed that the dynamics of both vehicles (system matrices and input statistics) and the parameters of both OCMs are are given. The corresponding state dynamics for time-delays can be developed by knowing their numerical values.

Based on the above given parameters and some matrix transformations, we determined the adaptive elements of the OCMs (Filter \& controller gains). By appropriate choices of system parameters, the Equations (5.14)-(5.16) can be used to solve for optimal controller gains $F_{1}^{*}$ and $F_{2}^{*}$, and equations (5.20)-(5.22) solve for optimal filter gains $\mathrm{K}_{1}$ and $\mathrm{K}_{2}$. 


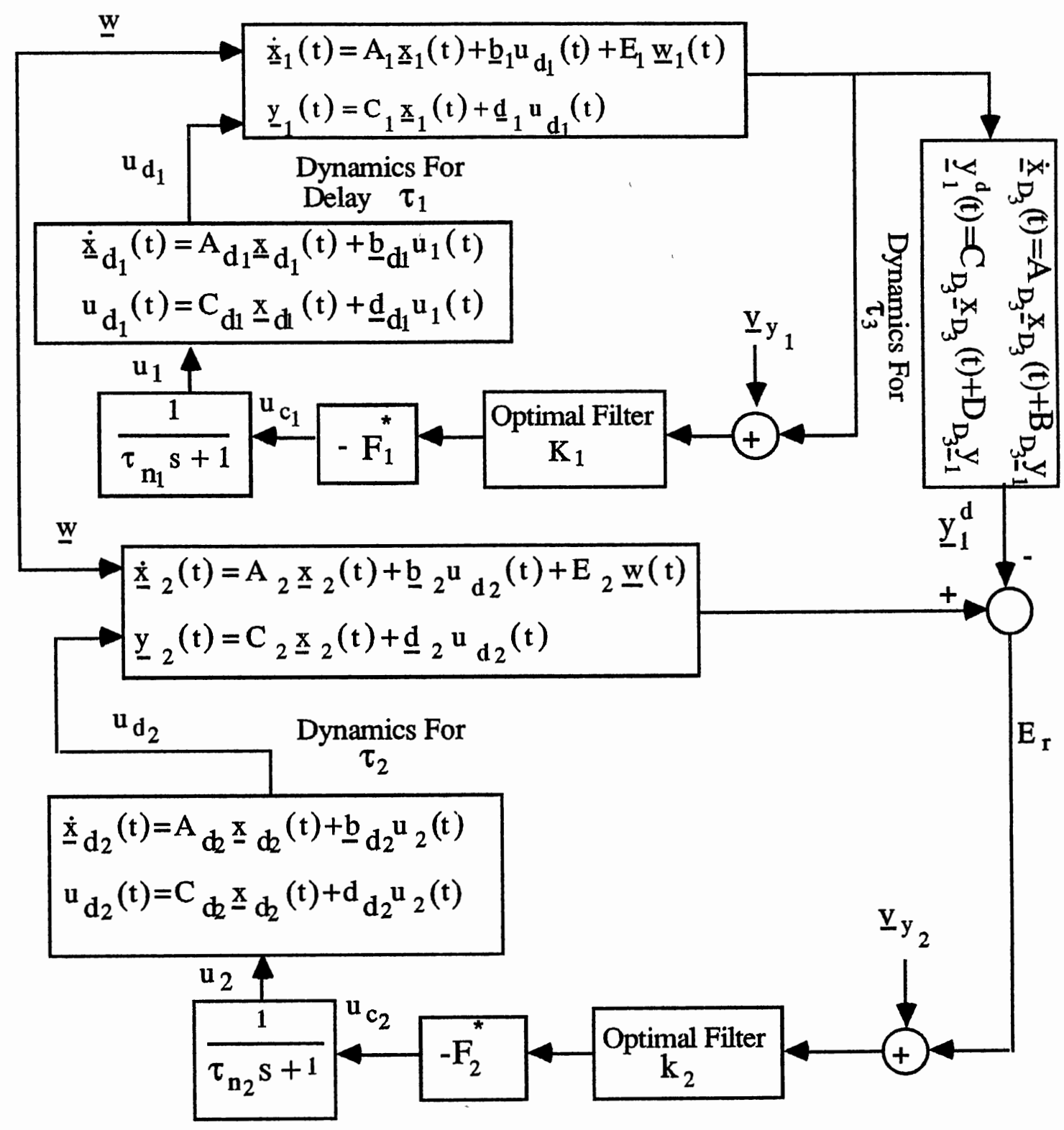

Figure 5.6. Reconfiguration Of Figure 5.1 


\section{CHAPTER VI}

\section{ANALYTICAL RESULTS}

Chapter V considered the formation flight task (a special class of networked flight simulator problems) as a dual OCM and represented its analytical solution in detail. In this chapter we discusse the effects of network delays on the pilot performance in a simplified formation flight task.

This chapter is organized as follows. Section 6.1 outlines the main simulation results obtained from a simplified formation flight task. In order to explain these results, sections 6.2-6.4 consider some simple optimal tracking problems. Section 6.5 considers some simple dual OCMs and summarizes their analytical results. Finally, section 6.6 concludes this chapter.

\subsection{Analytical Results of a Formation Flight Task}

This section considers the effects of time-delays (mainly intersimulator time delay $\tau_{3}$ ) on the human operator (pilot) performance in a simplified formation flight problem. Figure 6.1 shows a blockdiagram representation of the dual OCM for this problem. The vehicle dynamics diagrammed in Figure 6.2 represent a first approximation of the roll axis of an F-16 flying at $100 \mathrm{ft}$. at 250 knots together with a first order gust model appropriate to the vehicle speed and 
altitude. The gust state is filtered at $2.86 \mathrm{rad} / \mathrm{sec}$ by a first order filter. As indicated in Figure 6.1, the displayed variables are the error and error rate. The error is selected to be the roll angle (actual or delayed) mismatch between the two aircraft as displayed to the Wingman (second human operator). The disturbance intensity, $\mathrm{W}$, is chosen to guarantee an $\mathrm{rms}$ gust level of $3 \mathrm{deg} / \mathrm{s}$ and the control stick generates a $16 \mathrm{deg} / \mathrm{s}$ command for one pound of force input.

In the analysis of any of the experimental cases in this study, the cost functional of the form $J(u)=E\left\{e^{2}(t)+g \dot{u}^{2}(t)\right\}$ was taken for both OCMs (lead pilot and, wingman) in the dual OCM, with appropriate definition of the error quantity $e$ and the control input $u$. The normalized observation noises on error and error rate for both OCMs are adjusted to $-20 \mathrm{~dB}$ and the normalized motor noise is adjusted to $-25 \mathrm{~dB}$. The nominal values for motor lags $\tau_{n_{1}}=\tau_{n_{2}}=0.1 \mathrm{~s}$ for OCMs are adjusted by selecting the appropriate control rates, $g_{1}$ and $g_{2}$, equal to 0.0363 . A computer program has been developed [87] for implementing the dual OCM.

As shown in Figure 6.1, the error, e, can be chosen to be one of two things: 1) the difference between the roll angle of the second vehicle $\underline{y}_{2}$ and that of the first vehicle $\underline{y}_{1}$; or 2 ) the difference between $\underline{y}_{2}$ and the delayed output of the first vehicle, $\underline{y}_{1}^{d}$. The former is called the actual error and the latter is called the delayed error. 


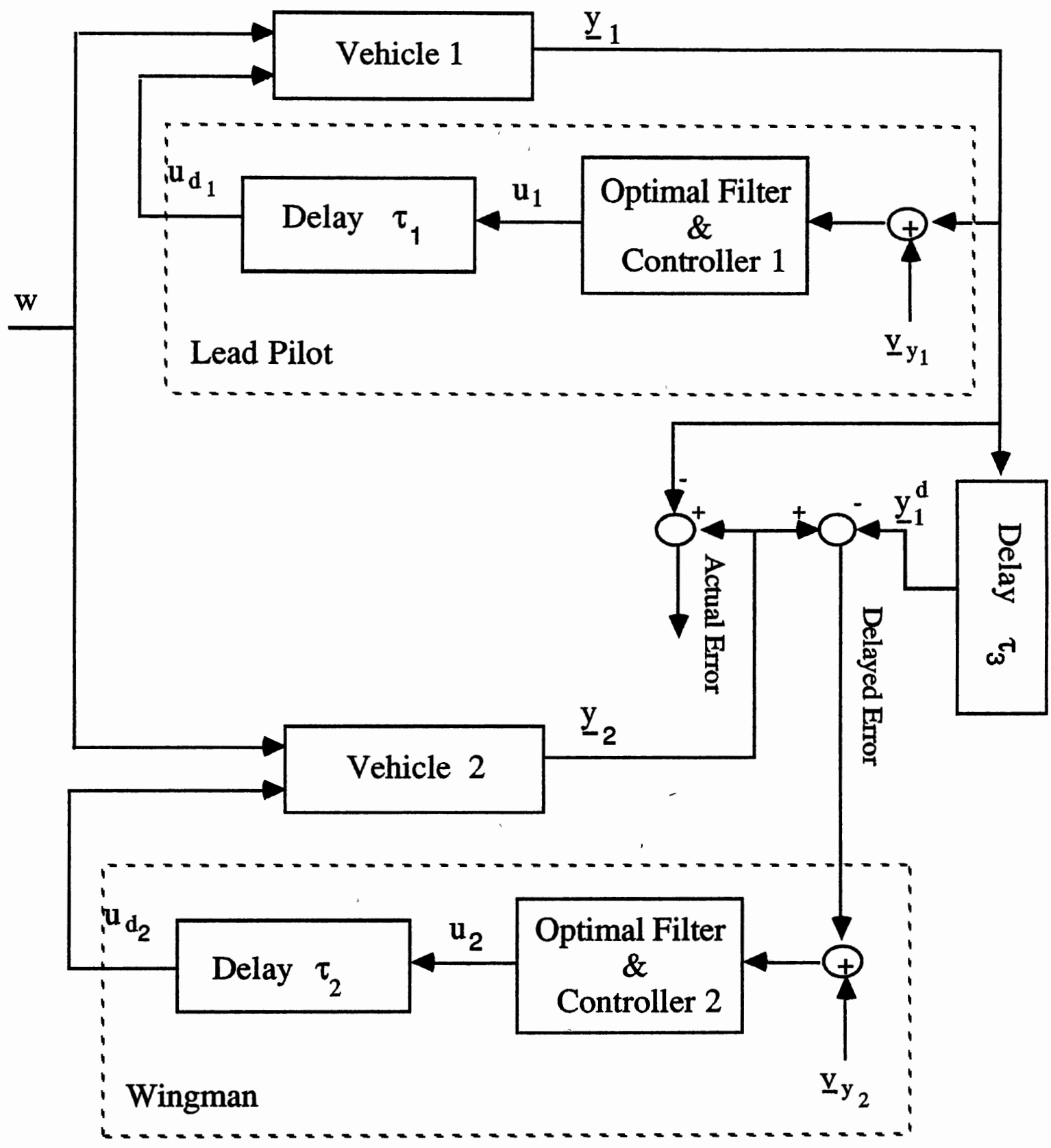

Figure 6.1 Dual OCM For a simplified Formation Flight Problem 


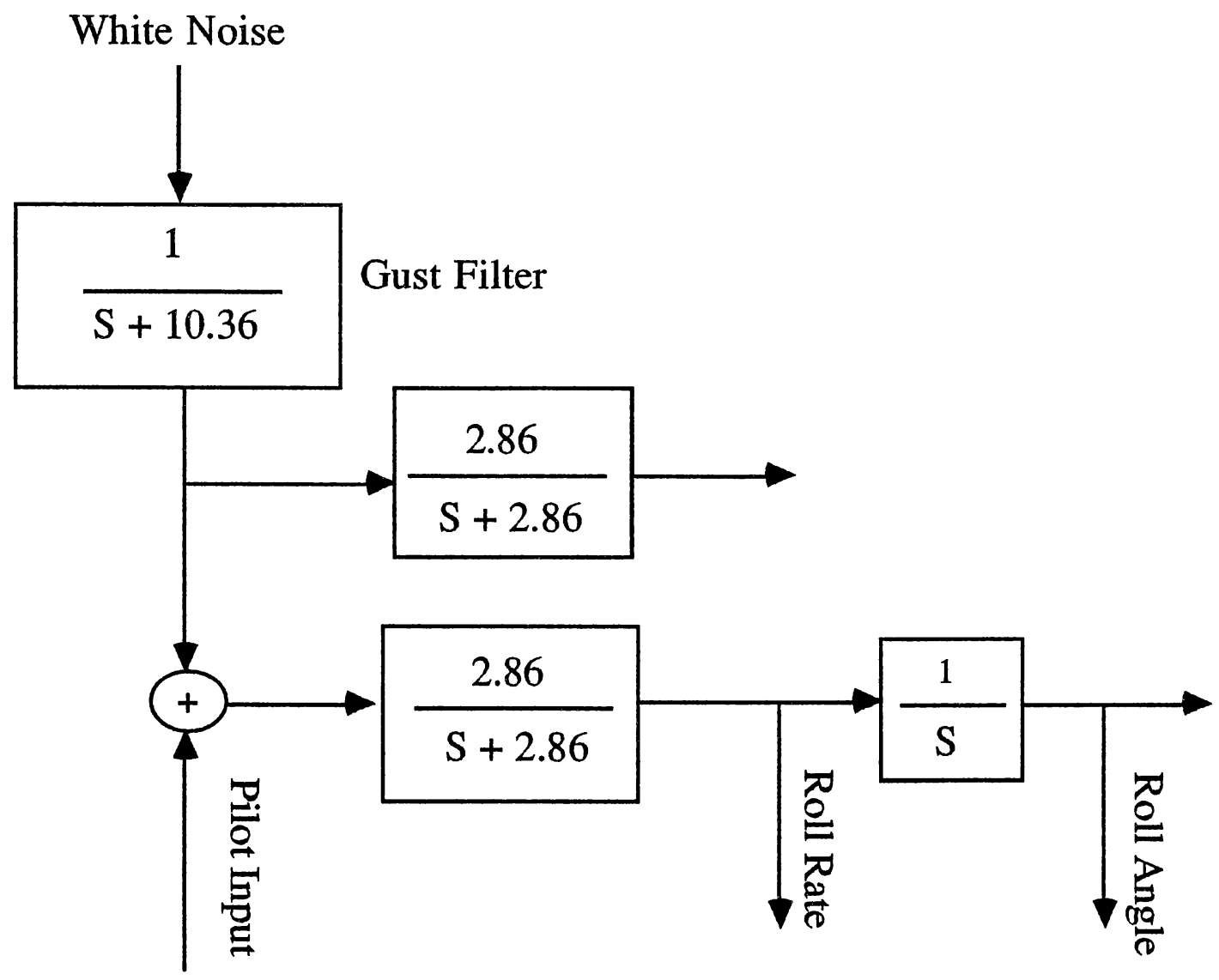

Figure 6.2 Vehicle Dynamics In The Dual OCM

In this study, the following notation is used extensively.

$D_{d}: \quad$ Delayed error while minimizing delayed error $A_{d}$ : Actual error while minimizing delayed error $\mathrm{D}_{\mathrm{a}}$ : Delayed error while minimizing actual error $A_{a}$ : Actual Error while minimizing actual error $\mathrm{H}_{1}(\mathrm{~s})$ : Transfer function linking $\mathrm{w}$ to $\underline{\mathrm{y}}_{1}$ $H_{1}^{d}(s)$ : Transfer function linking $w$ to $\underline{y}_{1}^{d}$ $\mathrm{H}_{2}$ (s): Transfer function linking $\mathrm{w}$ to $\underline{\mathrm{y}}_{2}$ $\tau_{3}^{\text {eq }}: \quad$ Stationary point of the error 
In this analysis, the main concern is to investigate the effects of inter-simulator delay $\tau_{3}$ on the overall system performance and to determine the possible range of the delayed variables. The impact of the delay $\tau_{3}$ on the overall system performance is illustrated in Figure 6.3. It is a plot of mean square error as a function of $\tau_{3}$, $0.001 \mathrm{~s} \leq \tau_{3} \leq 0.6 \mathrm{~s}$. Note that $\tau_{1}$ was set to $0.15 \mathrm{~s}$ and $\tau_{2}$ was set to $0.05 \mathrm{~s}$ and $0.15 \mathrm{~s}$. The top part of Figure 6.3 represents actual error and delayed error for the case where the delayed error is minimized. The bottom part of Figure 6.3 represents these errors when the actual error is minimized.

As shown in Figure 6.3, the delayed error drops initially to reach a minimum before increasing and the actual error goes up initially to reach a maximum before decreasing. These opposite trends of errors don't agree with our expectation of monotonically increasing errors. Furthermore, Figure 6.3 shows that the delayed error (actual error) will reach its minimum (maximum) later for larger $\tau_{2}$.

Figure 6.4 compares the errors illustrated in Figure 6.3. At the top of Figure 6.4, we see the actual and delayed errors while delayed error is minimized, and the bottom of this figure shows the errors when actual error is minimized. We should expect, as indicated in Figure 6.4, $D_{d} \leq D_{a}$ and $A_{a} \leq A_{d}$ for almost all $\tau_{3} \in[0.001,0.6]$, that delayed (actual) error while minimizing delayed (actual) error should be less than delayed (actual) error while minimizing actual (delayed) error. 

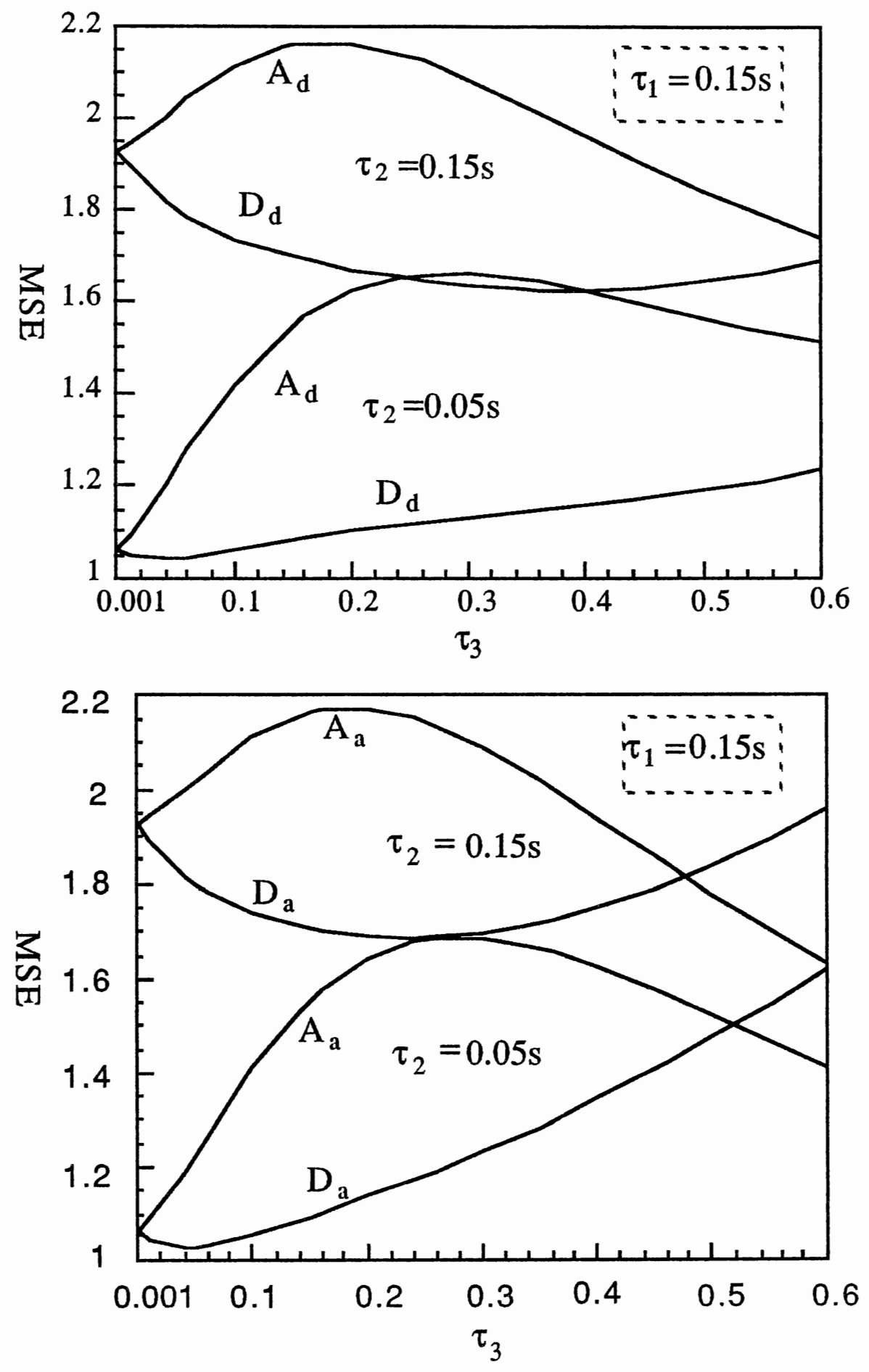

Figure 6.3 Effect Of Intra-simulator Delay On System Performance 

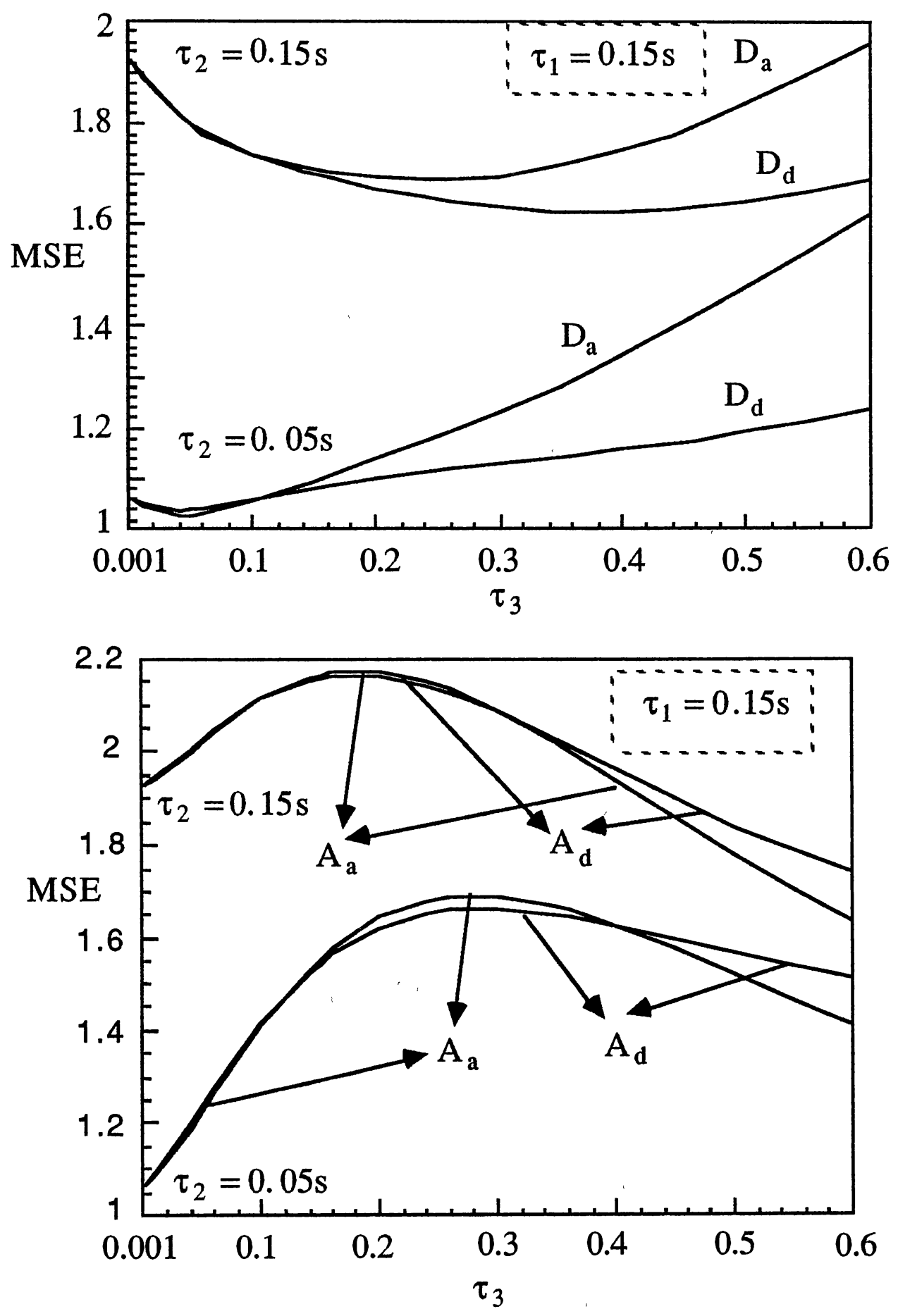

Figure 6.4. Comparison Of The Errors Plotted In Figure 6.3 
The basic conclusions of these experiments are as follows: 1) the errors are non-monotonic as a function of $\tau_{3}$, and 2) the stationary point (minimum or maximum) varies as a function of internal delays.

In order to check the above results and explore the effects of time delays on tracking performance, several test experiments have been made. In these tests the intra-simulator delay $\tau_{3}$ was varied over the range 0.0001 to $0.6 \mathrm{sec}$ and internal delays $\tau_{1}$ and $\tau_{2}$ were varied over the range from 0.05 to $0.55 \mathrm{sec}$. The results are illustrated in Figures 6.5 through 6.7 which give the mean square error versus system delays.

These Figures show three trends. First, if the first internal time delay $\tau_{1}$ is increased, while the second internal time delay $\tau_{2}$ is held fixed, the delayed error (actual error) is increased and will reach its minimum (maximum) sooner (later). In other words, for a fixed $\tau_{2}$, as $\tau_{1}$ is increased the minimum delayed error occurs at a smaller $\tau_{3}$ and the maximum actual error occurs at a larger $\tau_{3}$. Figures 6.5 illustrate this trend best.

Figure 6.5a represents a two-dimensional plot of the rms tracking error as a function of $\tau_{3}$ and $\tau_{1}$ for a fixed $\tau_{2}=0.25 \mathrm{~s}$. As seen, the delayed error drops initially and reaches its minimum at $\tau_{3}^{\mathrm{eq}}\left(\tau_{1}\right)$ before incresing. As $\tau_{1}$ increases, $\tau_{3}^{\mathrm{eq}}\left(\tau_{1}\right)$ becomes smaller. On the contrary, the actual error starts increasing and will reach a maximum at $\tau_{3}^{\text {eq }}\left(\tau_{1}\right)$ before decreasing. In this case, $\tau_{3}^{\text {eq }}\left(\tau_{1}\right)$ becomes bigger as $\tau_{1}$ increases. 
It might be helpful to see this trend in a three-dimensional plot. Figure $6.5 \mathrm{~b}$ displays the delayed error as a function of $\tau_{3}$ and $\tau_{1}$ for $\tau_{2}=0.05 \mathrm{~s}$ and $\tau_{2}=0.35 \mathrm{~s}$. For these two values of $\tau_{2}$, the corresponding actual errors are plotted in Figures 6.5c.
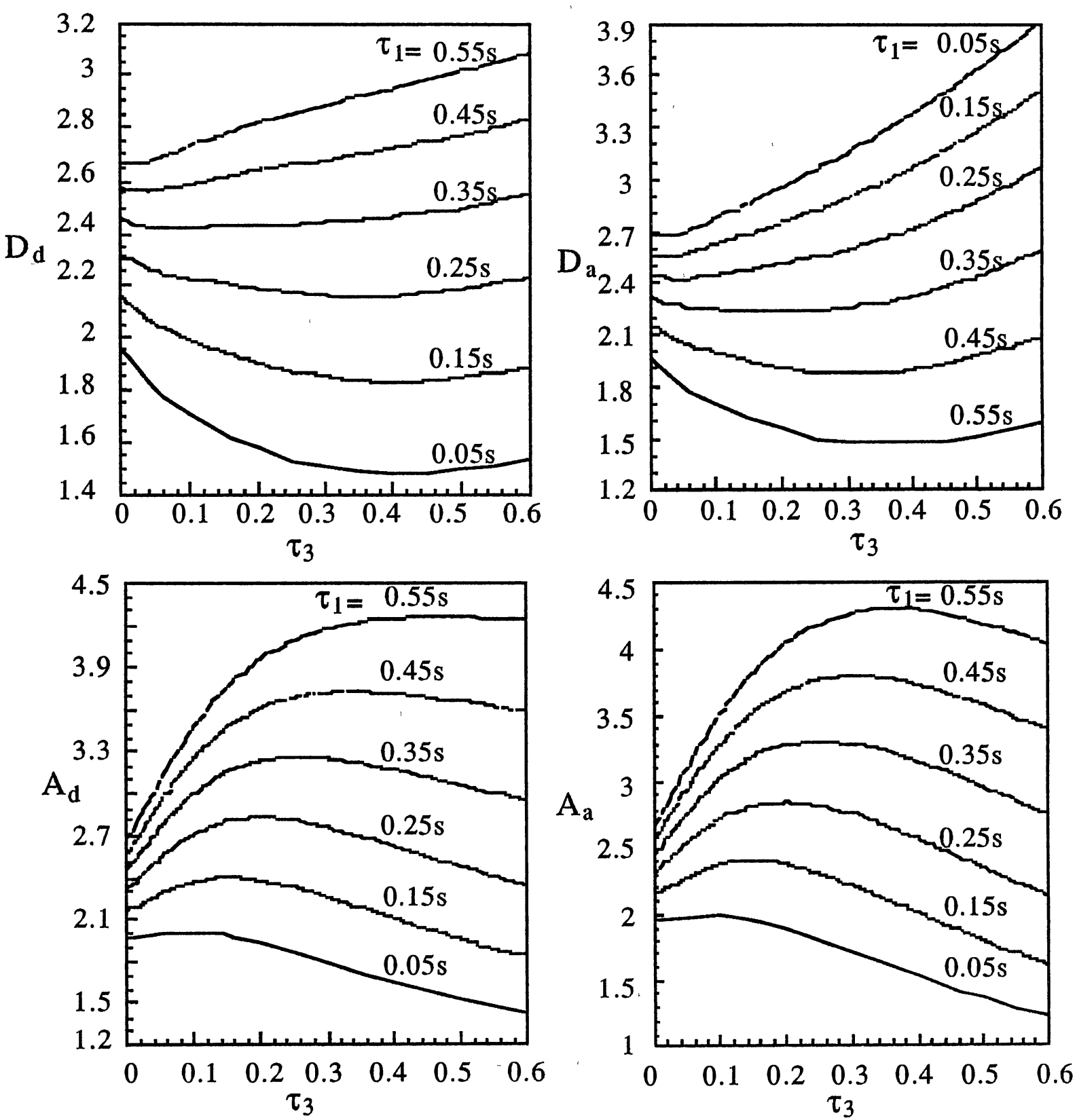

Figure 6.5a. The Effect of $\tau_{1}$ on Mean for a fixed $\tau_{2}=0.25 \mathrm{~s}$ 

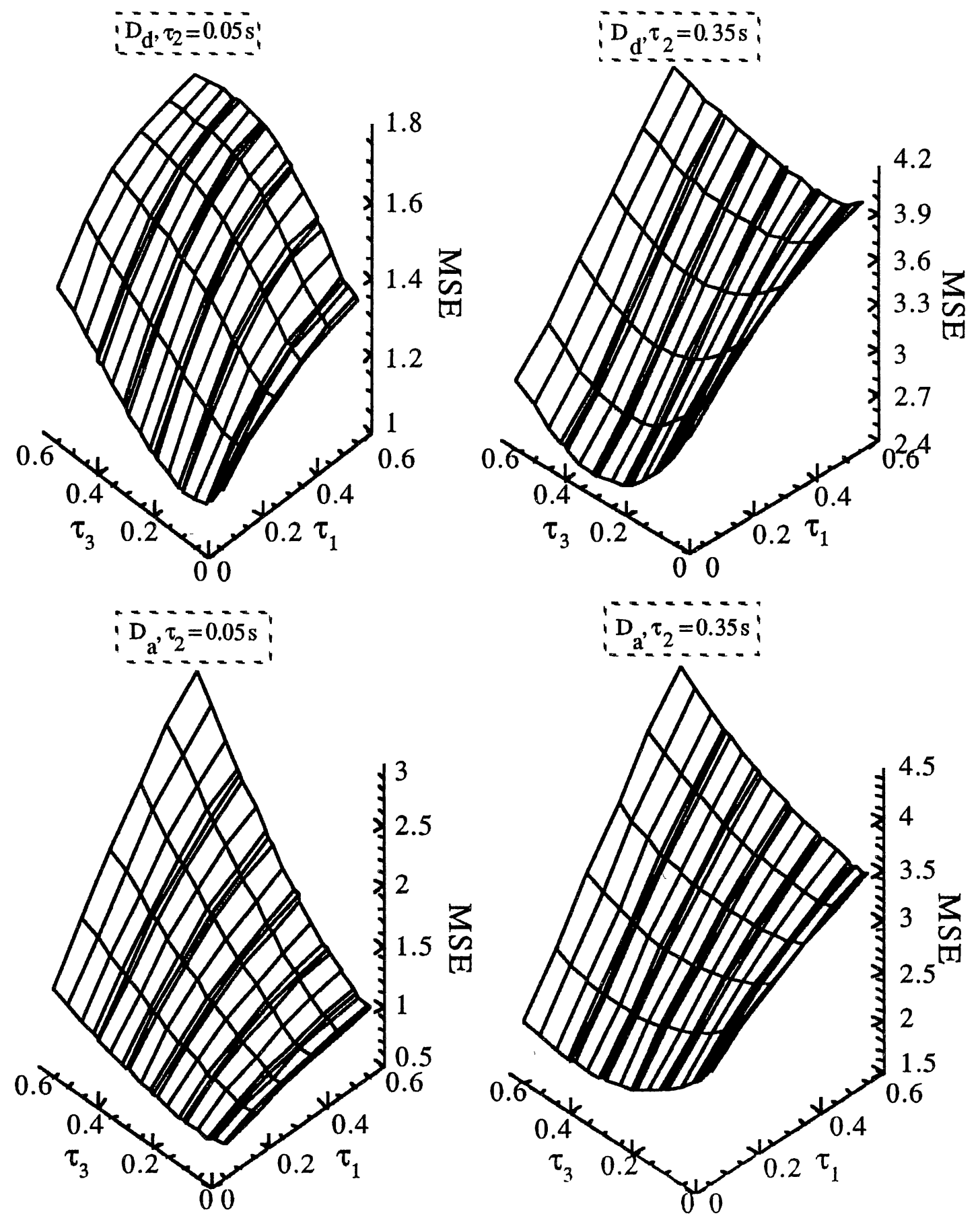

Figure $6.5 \mathrm{~b}$. The Effect Of $\tau_{1}$ On Mean Square Error 

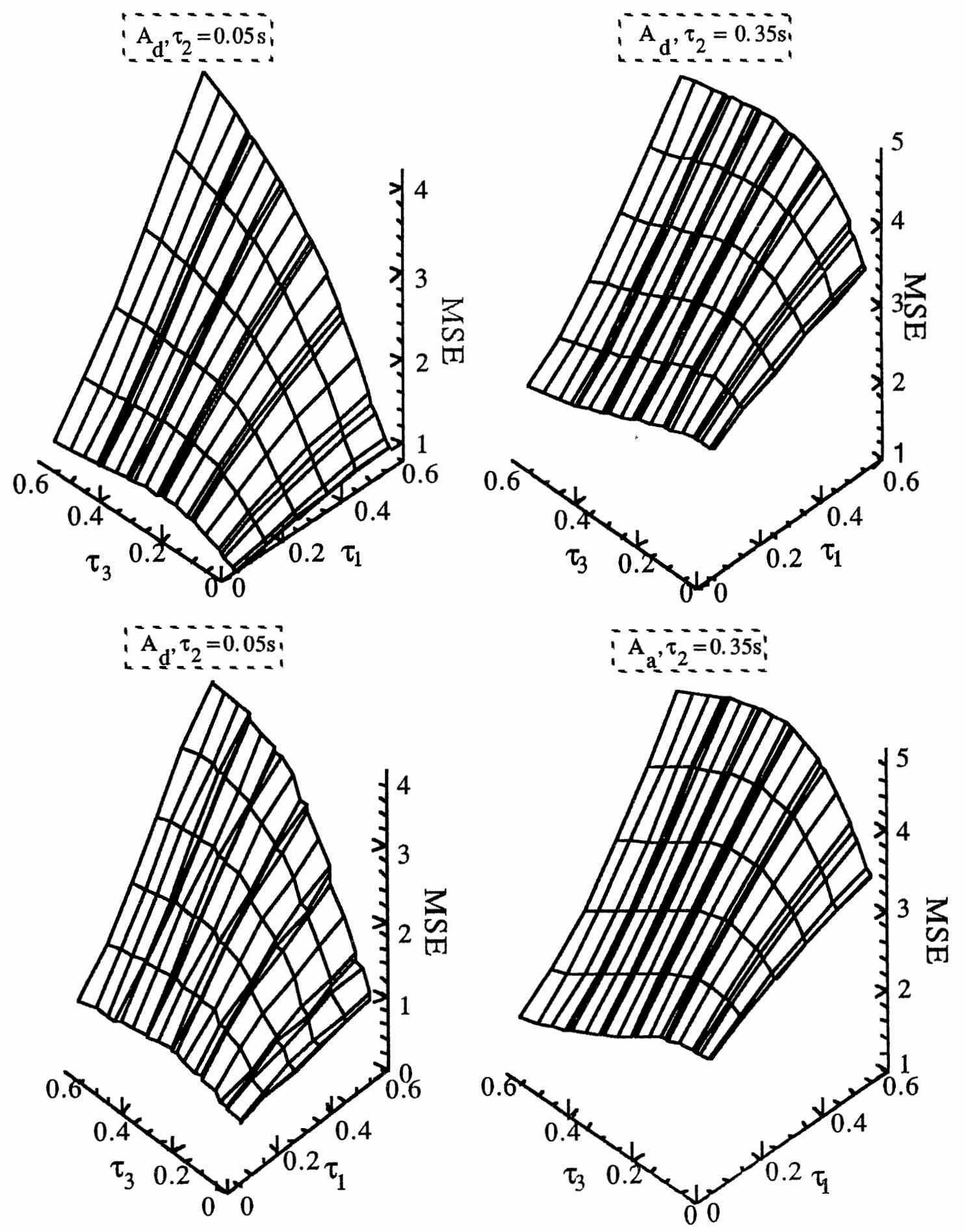

Figure 6.5c. The Effect Of $\tau_{1}$ On Mean Square Error 
These figures verify the trends observed in Figure 6.5a. The effect of $\tau_{1}$ on rms tracking error is best summarized in Figure 6.5d. This figure is obtained by cross-sectioning Figures $6.5 \mathrm{~b}$ and $6.5 \mathrm{c}$ at the stationary points. For example, the upper left of Figure 6.5d ilustrates the effect of $\tau_{1}$ on the delayed error while minimizing delayed error for a fixed value of $\tau_{2}=0.35 \mathrm{~s}$. As seen, the point at which the minimum of $D_{d}$ occurs, $\tau_{3}^{\text {eq }}$, becomes smaller as $\tau_{1}$ increases.
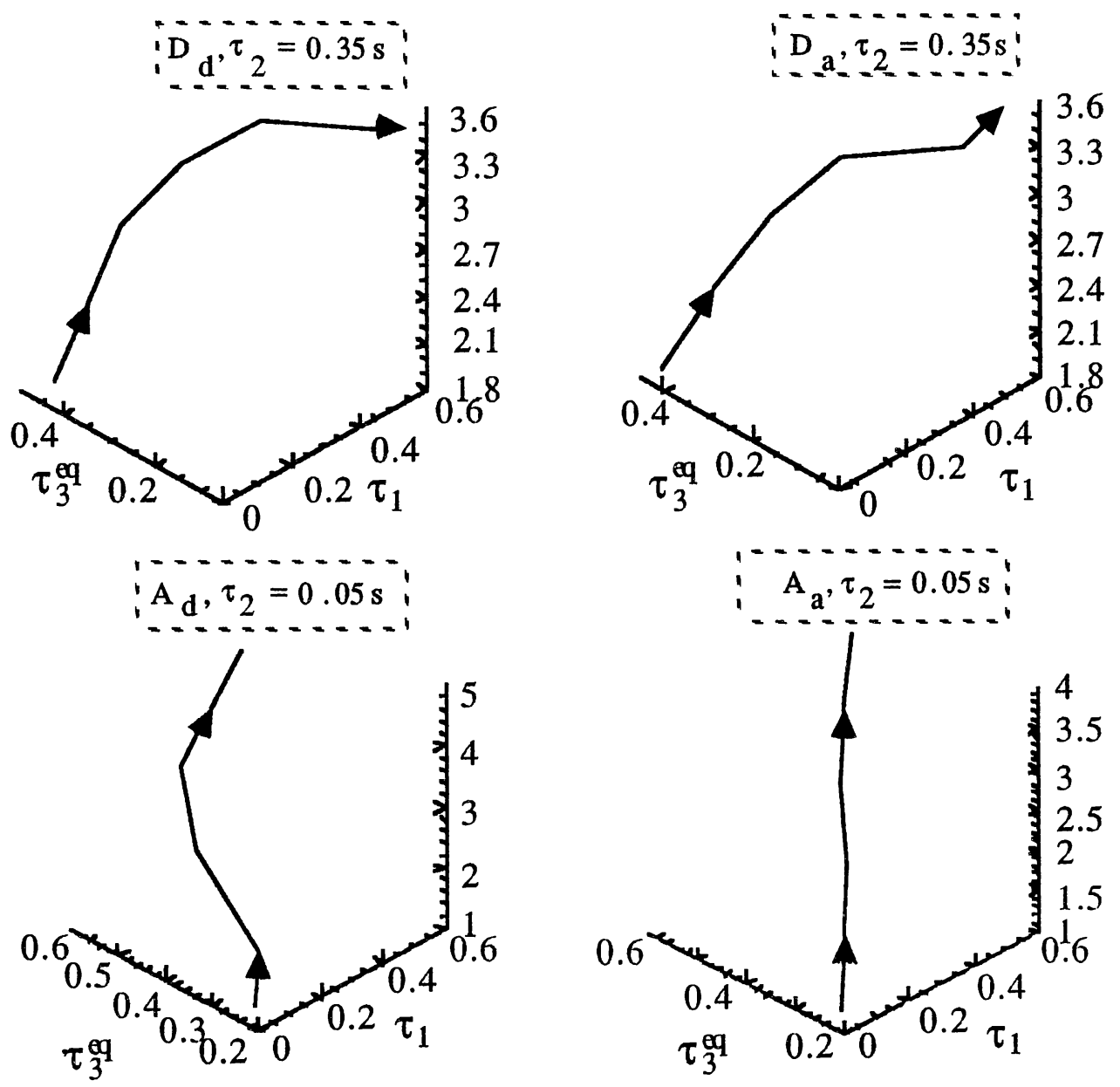

Figure 6.5d. The Effect Of $\tau_{1}$ On Mean Square Error 
Secondly, if $\tau_{2}$ is increased while $\tau_{1}$ is held fixed, then delayed (actual) error is increased and will reach its minimum (maximum) later (sooner). In other words, for any fixed $\tau_{1}$ as $\tau_{2}$ is increased the minimum delayed error occurs at a larger $\tau_{3}$, whereas the maximum actual error occurs at a smaller $\tau_{3}$. This trend is seen in Figures 6.6.

Figure 6.6a represents a two-dimensional plot of the rms tracking error as a function of $\tau_{3}$ and $\tau_{2}$ for a fixed $\tau_{1}=0.25 \mathrm{~s}$. As illustrated, the delayed error drops initially and reaches its minimum at $\tau_{3}^{\text {eq }}\left(\tau_{2}\right)$ before incresing. As $\tau_{2}$ increases, $\tau_{3}^{\text {eq }}\left(\tau_{2}\right)$ becomes bigger. Conversely, the actual error starts increasing and will reach a maximum at $\tau_{3}^{\text {eq }}\left(\tau_{2}\right)$ before decreasing. In this case, $\tau_{3}^{\text {eq }}\left(\tau_{2}\right)$ becomes smaller as $\tau_{2}$ increases.

Figure 6.6b displays the delayed error as a function of $\tau_{3}$ and $\tau_{2}$ for $\tau_{1}=0.05 \mathrm{~s}$ and $\tau_{1}=0.35 \mathrm{~s}$. For these two values of $\tau_{2}$, the corresponding actual errors are plotted in Figures 6.5c. These figures verify the trends observed in Figure 6.6a. The effect of $\tau_{2}$ on rms tracking error is best summarized in Figure 6.5d. This figure is obtained by cross-sectioning Figures $6.6 \mathrm{~b}$ and $6.6 \mathrm{c}$ at the stationary points. For example, the lower left of Figure 6.6d illustrates the effect of $\tau_{2}$ on the actual error while minimizing delayed error for a fixed value of $\tau_{1}=0.35 \mathrm{~s}$. As shown, the point at which the maximum of $D_{d}$ occurs, $\tau_{3}^{\text {eq }}$, becomes smaller as $\tau_{2}$ increases. 

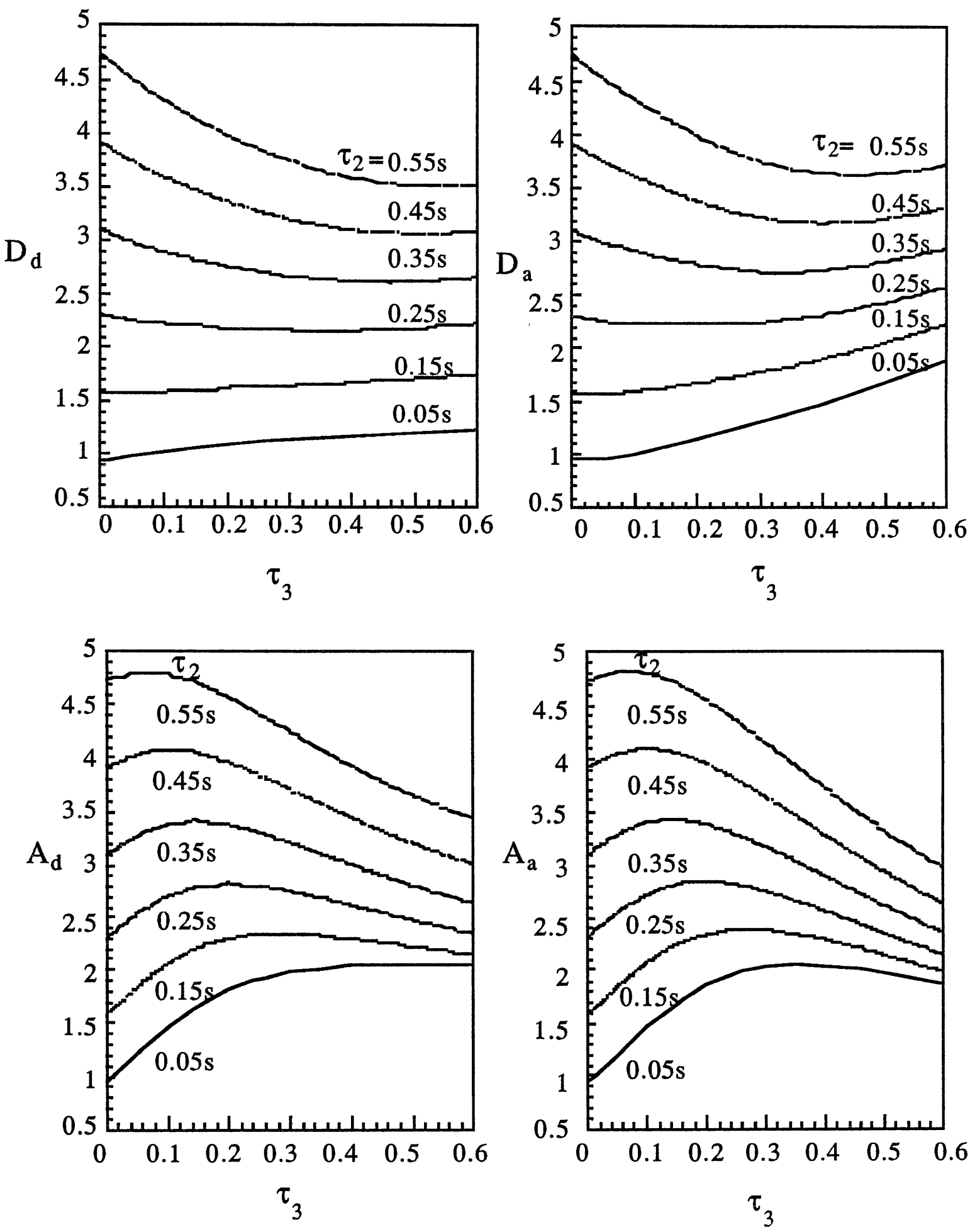

Figure 6.6a. The Effect of $\tau_{2}$ on Mean Square Error for a Fixed $\tau_{1}=0.25 \mathrm{~s}$ 

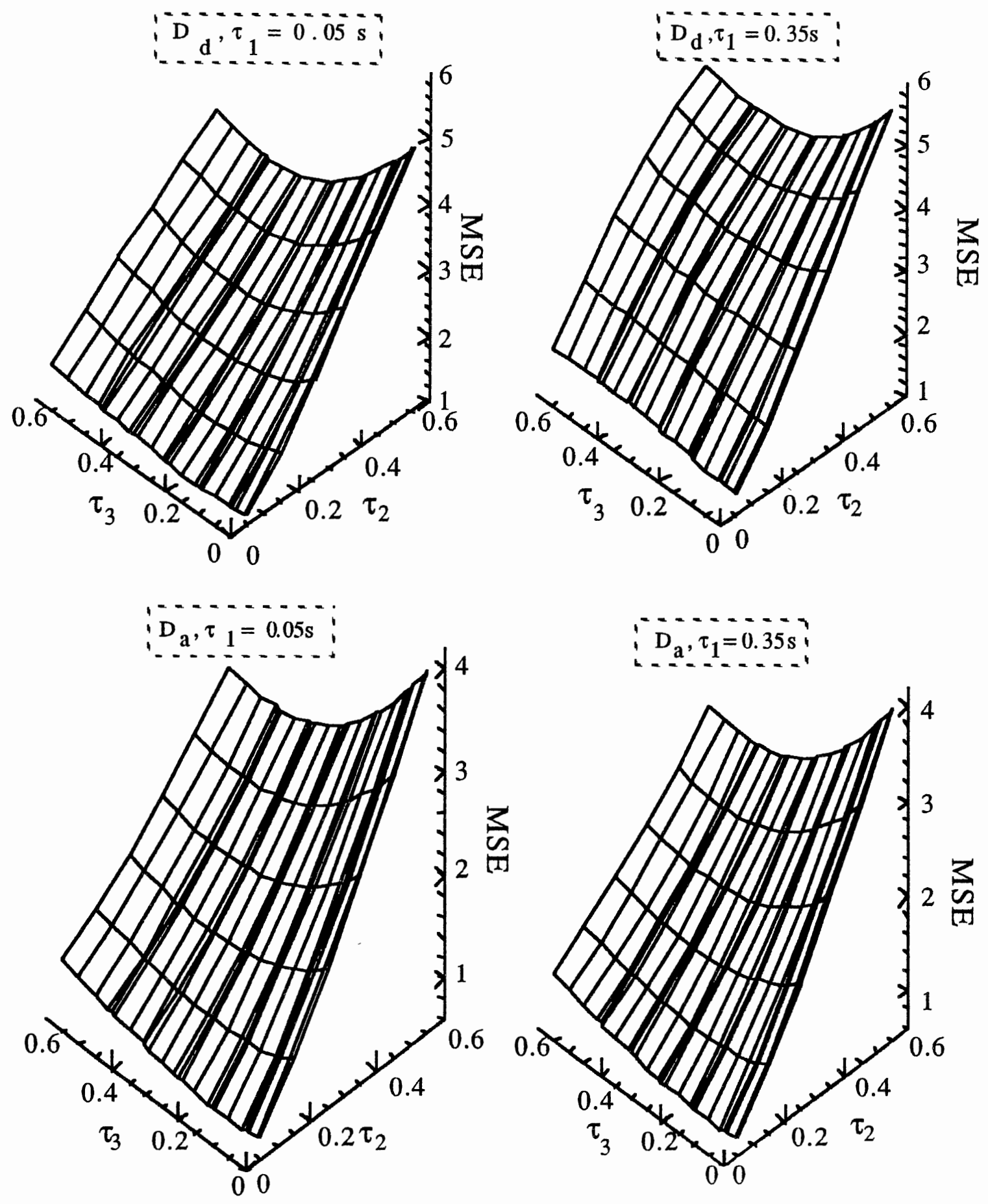

Figure 6.6b. The Effect Of $\tau_{2}$ On Mean Square Error 

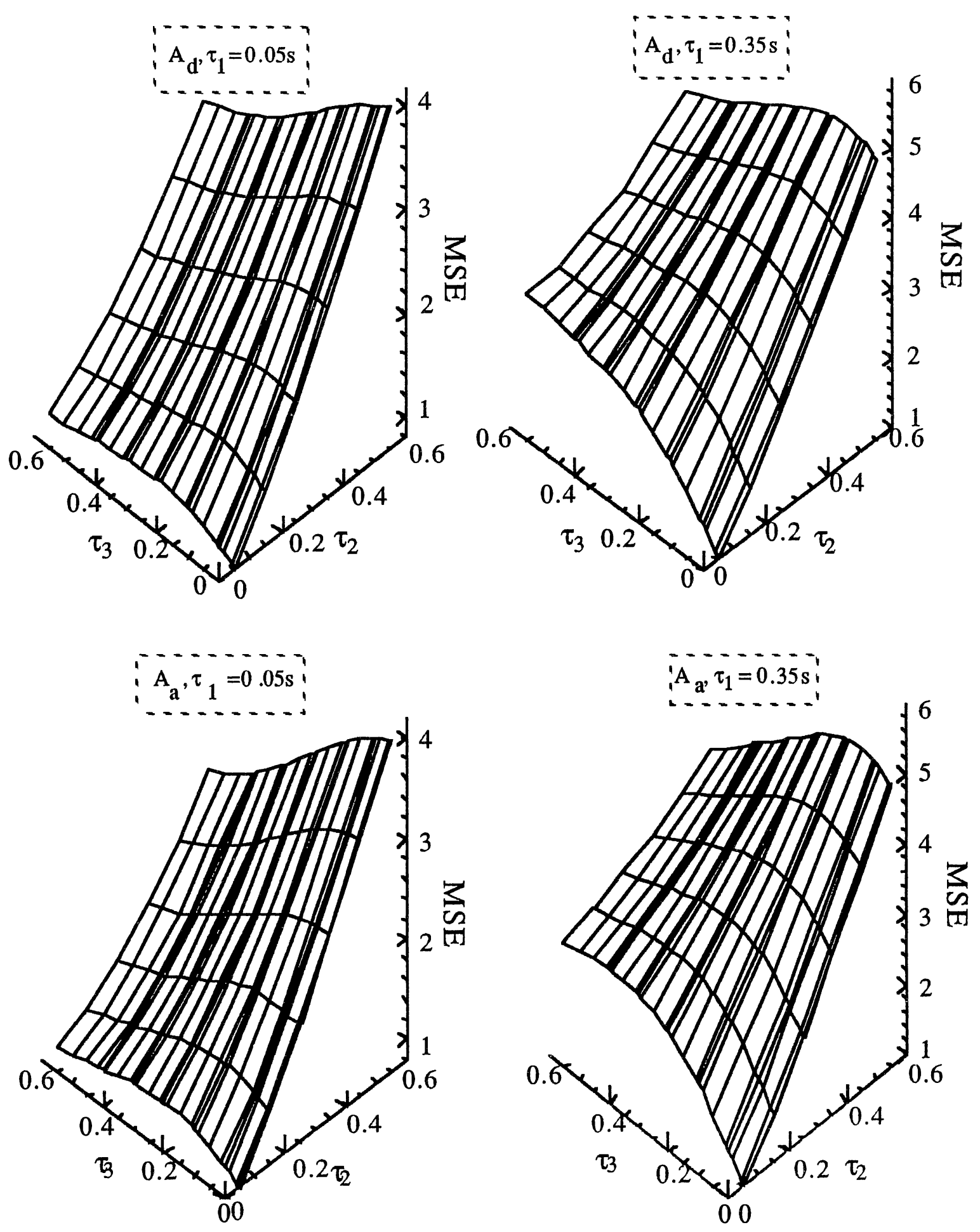

Figure 6.6c. The Effect Of $\tau_{2}$ On Mean Square Error 

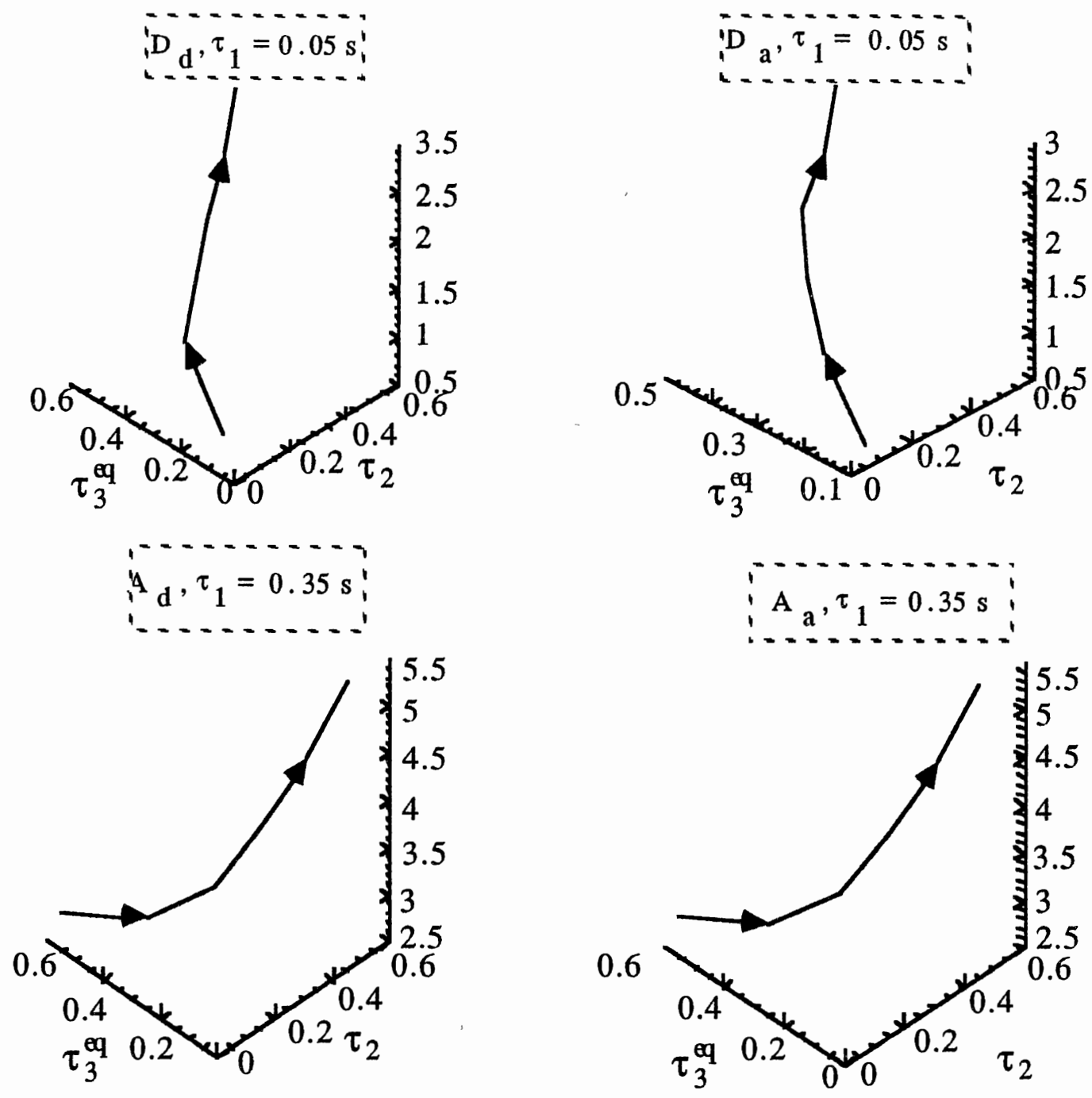

Figure 6.6d. The Effect Of $\tau_{2}$ On Mean Square Error 
Third, the internal time delay $\tau_{2}$ has a little more effect on mean square error (overall system performance) than $\tau_{1}$. Figure 6.7 illustrates this trend best. For example, the upper left of Figure 6.7 displays $D_{d}$ as a function of internal delays $\tau_{1}$ and $\tau_{2}$ for a fixed value of $\tau_{3}=0.15 \mathrm{~s}$. As we can see, for a fixed value of $\tau_{1}$ the rms tracking error, as a function of $\tau_{2}$, will increase much faster than that of the error for the case in which $\tau_{2}$ is held fixed.
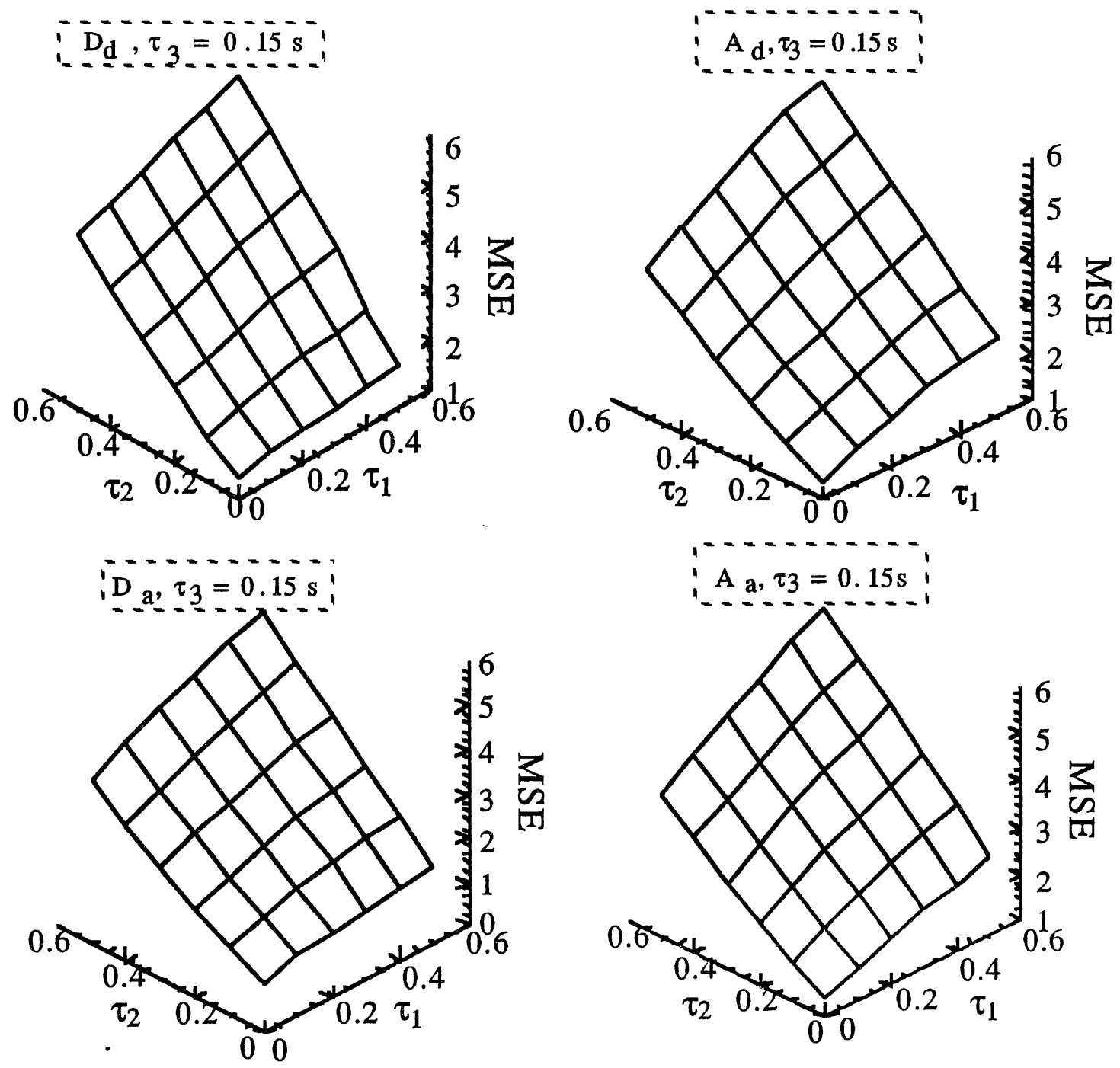

Figure 6.7. Mixed Effects of $\tau_{1}$ and $\tau_{2}$ on Mean Square Error 
We propose the following explanations for the trends mentioned above.

i) For rms delayed tracking error

The delays $\tau_{1}$ and $\tau_{3}$ make the reference tracking response, $\mathrm{y}_{1}^{\mathrm{d}}$, slow down. The time delay $\tau_{2}$ makes the response of the second OCM slow down (the plant model is made to respond slower as $\tau_{2}$ increases). Recalling that the dual OCM is basically a stochastic tracking problem (see chapter 5), we may achieve the best closedloop performance (the optimum rms delayed error) when there is a perfect match between the speed of plant and reference model. In other words, we may intuitively say that the sum of time delays $\tau_{1}$ and $\tau_{3}$ must be equal to the delay $\tau_{2}$, since the effect of $\tau_{3}$ on $y_{2}$ in this case can be ignored in comparison to its effect on reference tracking input.

In other words, when $\tau_{2}$ is increased, for a fixed $\tau_{1}$, it is expected that $\tau_{3}$ becomes larger in order to reach an optimum tracking performance. In addition, when $\tau_{1}$ increases, for a fixed $\tau_{2}$, we expect $\tau_{3}$ to decrease for achieving a minimum rms tracking error.

ii) For rms actual tracking error

In this case, the reference tracking signal is the output of the first plant, $y_{1}$. It becomes slower as $\tau_{1}$ increases. The delays $\tau_{2}$ and $\tau_{3}$ slow down $y_{2}$, the output of the second plant. The actual tracking error initially goes up and and reaches its maximum before 
decreasing. We expect that the maximum point will be as small as possible in order to have better performance. To do so, the sum of $\tau_{2}$ and $\tau_{3}$ should approximately match $\tau_{1}$. Therefore, for a fixed $\tau_{1}$, as $\tau_{2}$ increases, we expect the error to decrease as a function of $\tau_{3}$, and when $\tau_{1}$ increases, for a fixed $\tau_{2}$, we expect the error to increase as a function of $\tau_{3}$.

In order to comment on the frequency response, which can explain the cause-and-effect relationship between delays and rms tracking errors, sections 6.2 through 6.4 consider different simple tracking cases and quantify the results which are summarized below.

To summarize, the time delays have a consistent and significant effect on the rms tracking errors. These errors are less sensitive to the internal delay $\tau_{1}$. In other words, the rms tracking errors are more impacted by the internal time delay $\tau_{2}$. The point at which the minimum (maximum) rms tracking error occurs, as a function of $\tau_{3}$, increases (decreases) with increasing (decreasing) $\tau_{2}$ and decreases (increases) with increasing (decreasing) $\tau_{1}$.

\subsection{A Simple Optimal Tracking Problem}

Section 6.1 considered the effects of time delays on pilot performance in a formation flight task (see Figure 6.1) and concluded that the optimal delayed tracking error, as a function of intersimulator time delay $\tau_{3}$, drops initially to reach a minimum before increasing. Furthermore, it has been shown that if we hold the first OCM time delay fixed and increase the second OCM time delay the 
delayed tracking error is increased and reaches its minimum at a larger $\tau_{3}$.

To investigate this trend, we considered a simple optimal stochastic tracking problem illustrated in Figure 6.8. The upper block diagram represents the reference model with external disturbance input $\mathrm{w}$, and the lower block diagram represents the plant whose output tracks the output of the reference model. The plant is disturbed by the same external input w. Both reference model and plant are represented by first order systems with model parameters $\tau_{3}$ and $\tau_{2}$ respectively.

Reference model

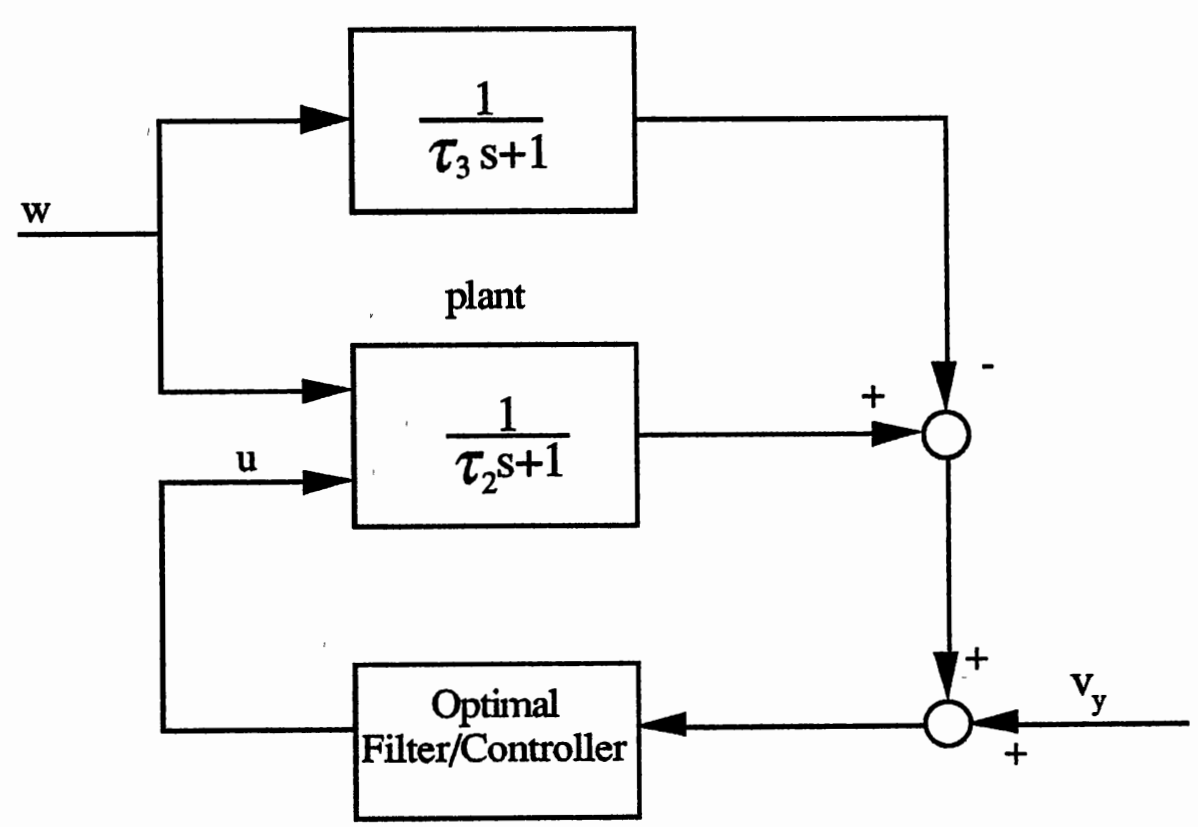

Figure 6.8. Simple optimal tracking problem 
The optimal mean square tracking errors versus $\tau_{3}$ are plotted in Figure 6.9 for different values of $\tau_{2}=0.15,0.25,0.35,0.45$ and 0.55 . In other words, Figure 6.9 shows the effect of $\tau_{2}$ on the plant performance. The error as a function of $\tau_{3}$ decreases initially and reaches its minimum at $\tau_{2}=\tau_{3}$ before increasing and reaching its steady state value (not shown in Figure 6.9). Appendix C presents a detailed derivation of this problem and shows theoretically the nonmonotonic property of the rms tracking error.

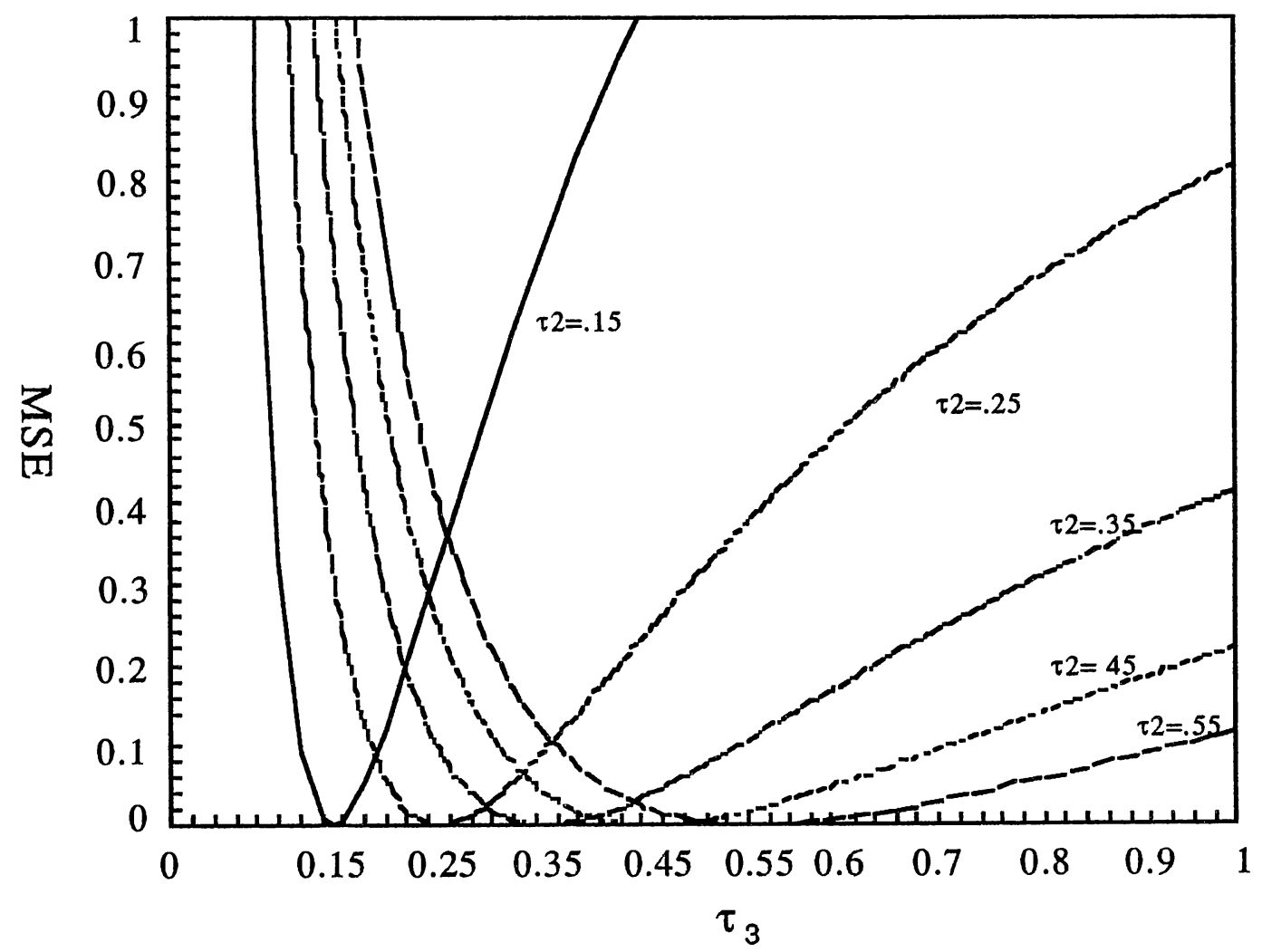

Figure 6.9. The effect of plant parameter $\tau_{2}$ on optimal mean square tracking error 
This is exactly the same trend which is observed for the formation flight task experiment. As $\tau_{2}$ is increased the optimal tracking error is increased and will reach its minimum later (at larger $\tau_{3}$ ). The reason why this trend happens may be stated as follows. As we increase $\tau_{2}$, the plant will react more slowly, and its response cannot track the desired output. Therefore, we observe a larger tracking error. For a fixed $\tau_{2}$, as $\tau_{3}$ increases, the reference model becomes slower, which yields a smaller tracking error, since the plant is able to track the output of reference model. At $\tau_{2}=\tau_{3}$ the error becomes zero, since we have a perfect match. In this case the optimal feedback will be zero, since the plant and the reference model match.

For $\tau_{3}$ bigger than $\tau_{2}$, the plant is responding to the same disturbance faster. Therefore the error starts to rise. As $\tau_{3}$ becomes much bigger than $\tau_{2}$, the output of the reference model is effectively constant, and the tracking problem is reduced to an optimal regulator problem with an external input $w$. The MSE in this case is exactly the variance of the plant, which is independent of $\tau_{3}$. This variance will increase as $\tau_{2}$ decreases.

As discussed in chapter 5, the formation flight task, in which the wingman tries to mimic the ideal pilot, is itself a tracking problem in a complex form. The trends mentioned above and fully discussed in section 6.1 are related to the nature of the general tracking problem. 


\subsection{A Simple Tracking Problem with Inter-simulator Delay}

There have been two analytical results of the networked simulator formation flight problems (discussed fully in section 6.1) which were unexpected and initially puzzling. The first was that the error perceived by the wingman (labeled "Delayed Error" in Figure 6.1) decreases initially as the inter-simulator time delay $\tau_{3}$ increases (see Figure 6.3). This non-monotonic behavior was considered in section 6.2 where a simple optimal stochastic tracking problem was applied to illustrate why this behavior occurs.

The second unexpected result was that the actual tracking error between the lead position and the wingman (labeled "Actual Error" in Figure 6.1) behaves in an opposite way than does the delayed error, as indicated in Figure 6.3. The mean square delayed error is decreasing while the mean square actual error is increasing.

The objective of this section is to illustrate, with a simple example, why this behavior occurs. The example is a simple optimal tracking problem depicted in Figure 6.10. This problem is similar to the one used in section 6.2, except that an inter-simulator delay $\tau_{3}$ has been added. In Figure 6.10, the upper block diagram represents the reference model with external disturbance input $w(t)$, and the lower block represents the plant model. The plant is disturbed by the same external input $w(t)$. Both the reference model and the plant are represented by first order systems with model parameters $\tau_{1}$ and $\tau_{2}$ respectively. 


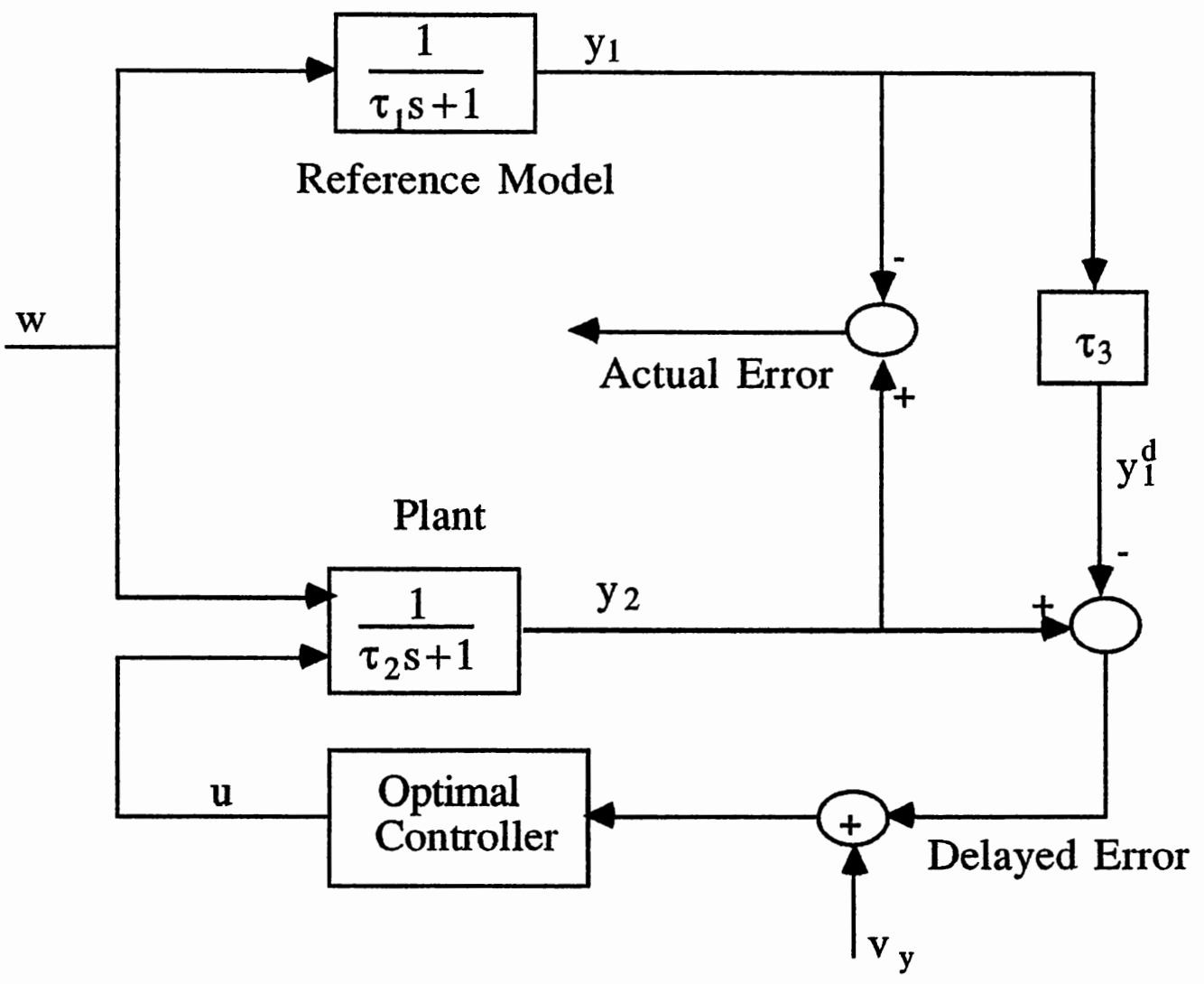

Figure 10. Simple Optimal Tracking Problem With An Intra-Simulator Time Delay $\tau_{3}$

In this problem, the error can be chosen from the difference between the output of the plant, $y_{2}$, and that of the reference model, $y_{1}$, or from the difference between $y_{2}$ and the delayed-output of the reference model, $y_{1}^{d}$. The former is called the actual error and the latter is called the delayed error. As in the previous sections, the following notation is used extensively. 
$D_{d}$ - Delayed error while minimizing delayed error

$A_{d}$ - Actual error while minimizing delayed error

$D_{a}$ - Delayed error while minimizing actual error

$A_{\mathbf{a}}$ - Actual error while minimizing actual error

$\mathrm{H}_{1}(\mathrm{~s})$ - Transfer function linking $\mathrm{w}$ to $\mathrm{y}_{1}$

$H_{1}^{d}(s)$ - Transfer function linking $w$ to $y_{1}^{d}$

$\mathrm{H}_{2}$ (s) - Transfer function linking $w$ to $\mathrm{y}_{2}$

Three cases have been considered with three different combinations of values for $\tau_{1}$ and $\tau_{2}$, and with $\tau_{3}$ ranging between 0 and 0.25 seconds for each case. Here, we will discuss only one case, since it illustrates the basic pattern.

CASE 1. $\tau_{1}=0.25, \tau_{2}=0.15, \tau_{3}=0,0.05,0.1,0.15,0.25$

Figure 6.11 displays the actual error and the delayed error for the case where the delayed error is to be minimized. Notice that the delayed error increases while the actual error decreases with $\tau_{3}$. This pattern is similar to that found in the dual OCM problem (see Figure $6.3)$.

The effect illustrated in Figure 6.11 can be understood by looking at the behavior of some of the transfer functions. Figure 6.12 represents the magnitude and phase plots of $H_{1}^{d}(s)$. As $\tau_{3}$ increases, $\mathrm{H}_{1}^{\mathrm{d}}(\mathrm{s})$ will have more phase lag due to pure intra-simulator delay, $\tau_{3}$.. The gain and phase plots of $\mathrm{H}_{2}(\mathrm{~s})$, are depicted in Figures 6.13, for the above different values of $\tau_{3}$. Figure 6.13a represents the frequency plots when the delayed error is minimized, and Figure 
$6.13 \mathrm{~b}$ represents the same plots for the case in which the delayed error is minimized.

Keep in mind that since we are minimizing $\left(\mathrm{y}_{2}-\mathrm{y}_{1}^{\mathrm{d}}\right)$ the optimal controller will attempt to make $\mathrm{H}_{2}(\mathrm{~s})$ match $\mathrm{H}_{1}^{\mathrm{d}}(\mathrm{s})$; if they are equal, $y_{2}$ will equal $y_{1}^{d}$. If $H_{2}(s)$ matches $H_{1}(s)$, then $y_{2}$ will equal $\mathrm{y}_{1}$.

By comparing the gain plots of Figure 613 with the gain plot of Figure 6.12, we can see that as $\tau_{3}$ increases, the gain of $\mathrm{H}_{2}(\mathrm{~s})$ is approaching the gain of $\mathrm{H}_{1}(\mathrm{~s}$ ) (which is the same as the gain of $\left.H_{1}^{d}(s)\right)$. Moreover, as we compare the phase plots of Figure 13 with the phase plot of Figure 6.12, it is seen that the phase of $\mathrm{H}_{2}(\mathrm{~s})$ is moving toward that of $H_{1}(s)$ (which is equal to the phase of $H_{1}^{d}(s)$ when $\tau_{3}=0$ ). These trends say that the actual error as a function of $\tau_{3}$ has to drop initially.

The delayed error $\left(D_{d}\right.$ or $\left.D_{a}\right)$ behaves differently; it increases monotonically (see Figure 6.11). The reason why the delayed error is monotonically increasing may be stated as follows: by increasing $\tau_{3}$, although the gain of $\mathrm{H}_{2}(\mathrm{~s})$ is approaching that of $\mathrm{H}_{1}^{\mathrm{d}}(\mathrm{s})$, we still have more phase lag in $\mathrm{H}_{1}^{\mathrm{d}}(\mathrm{s})$ than that of in $\mathrm{H}_{2}(\mathrm{~s})$. It means that even though $\mathrm{y}_{2}$ is getting closer to $\mathrm{y}_{1}$, as $\tau_{3}$ increases, the phase lag imposed by the pure time delay $\tau_{3}$ can not be fully compensated for. 

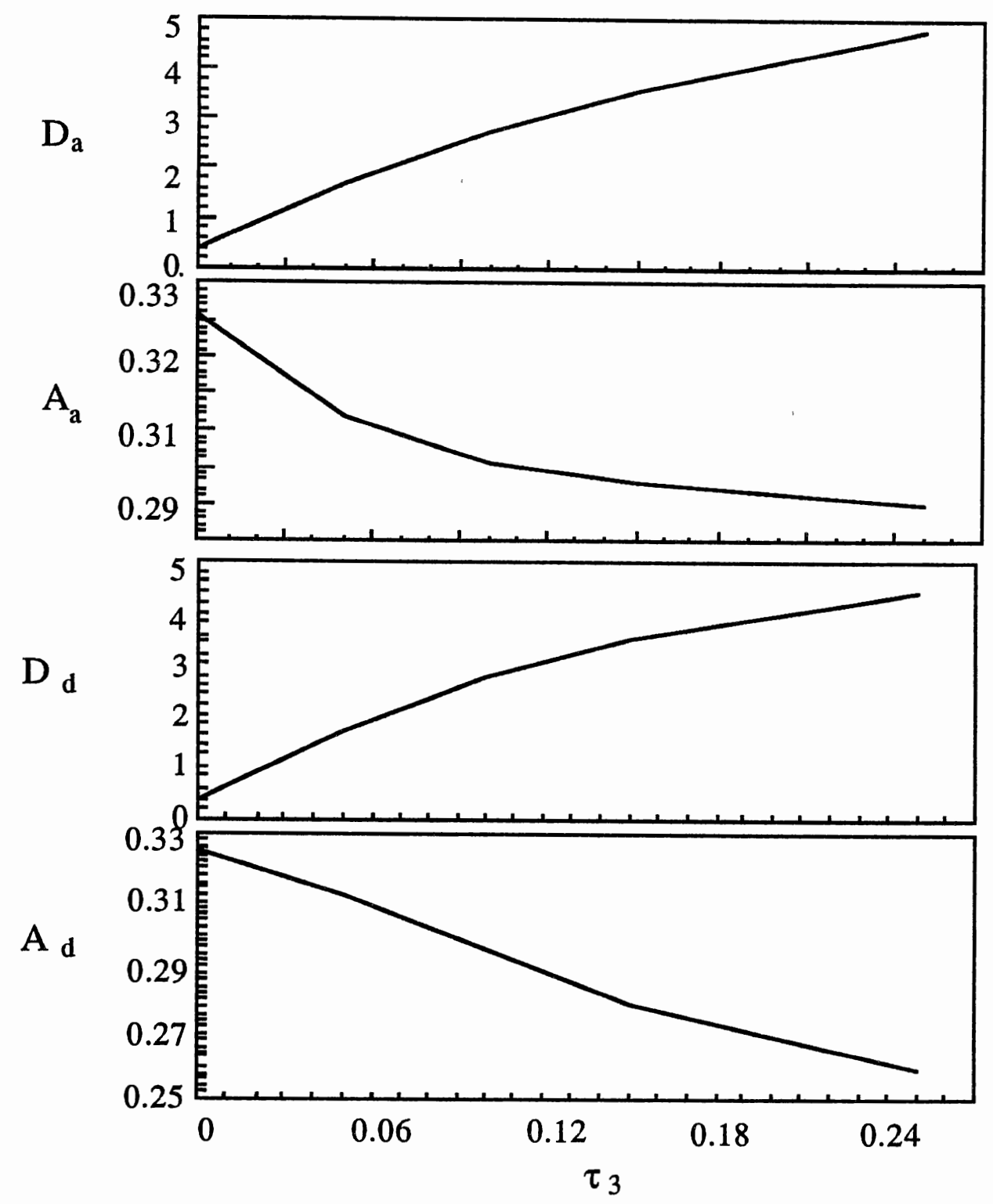

Figure 6.11. Actual Error And Delayed Error Versus $\tau_{3}$ 

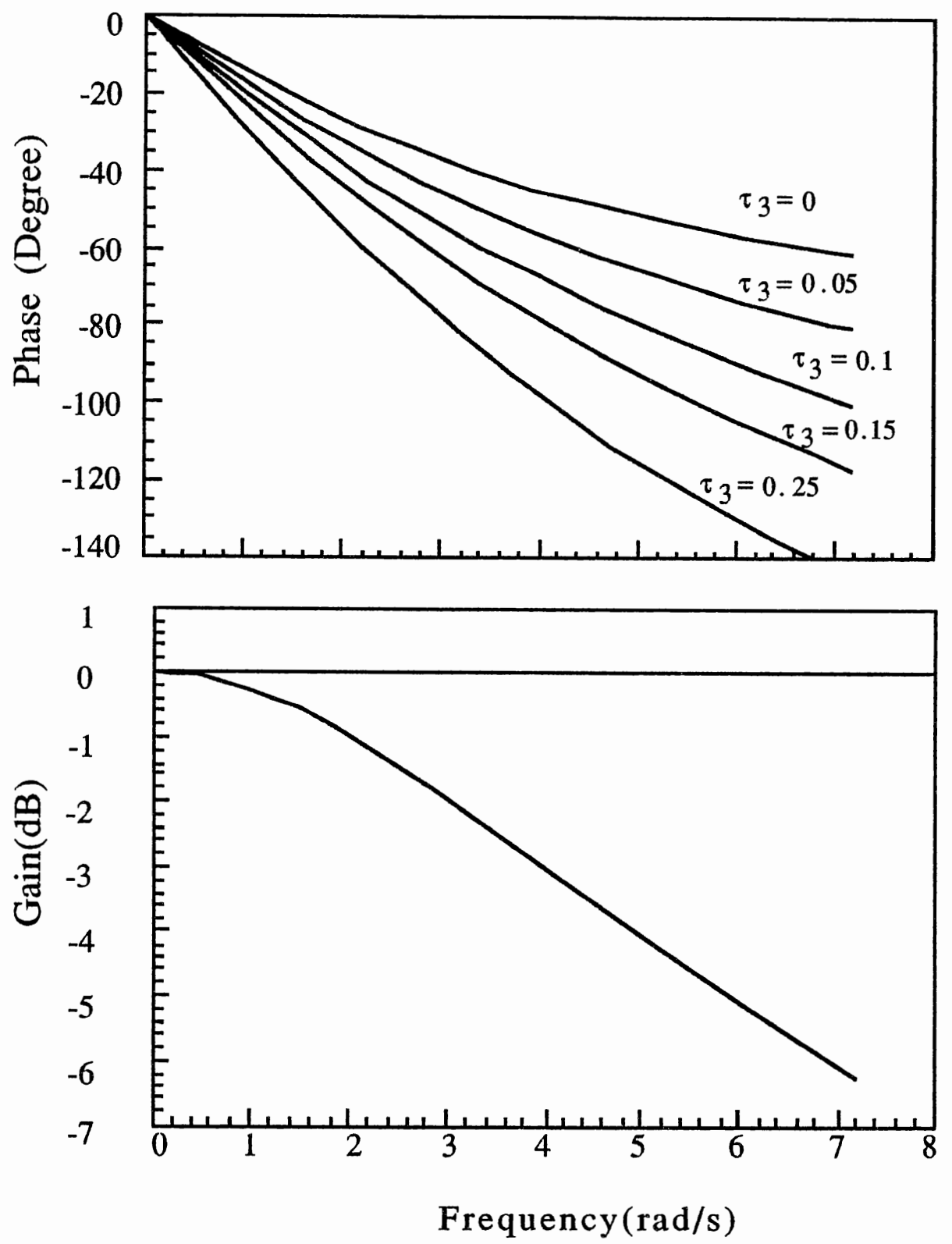

Figure 6.12. Magnitude And Phase Of $H_{1}^{d}(s)$ 

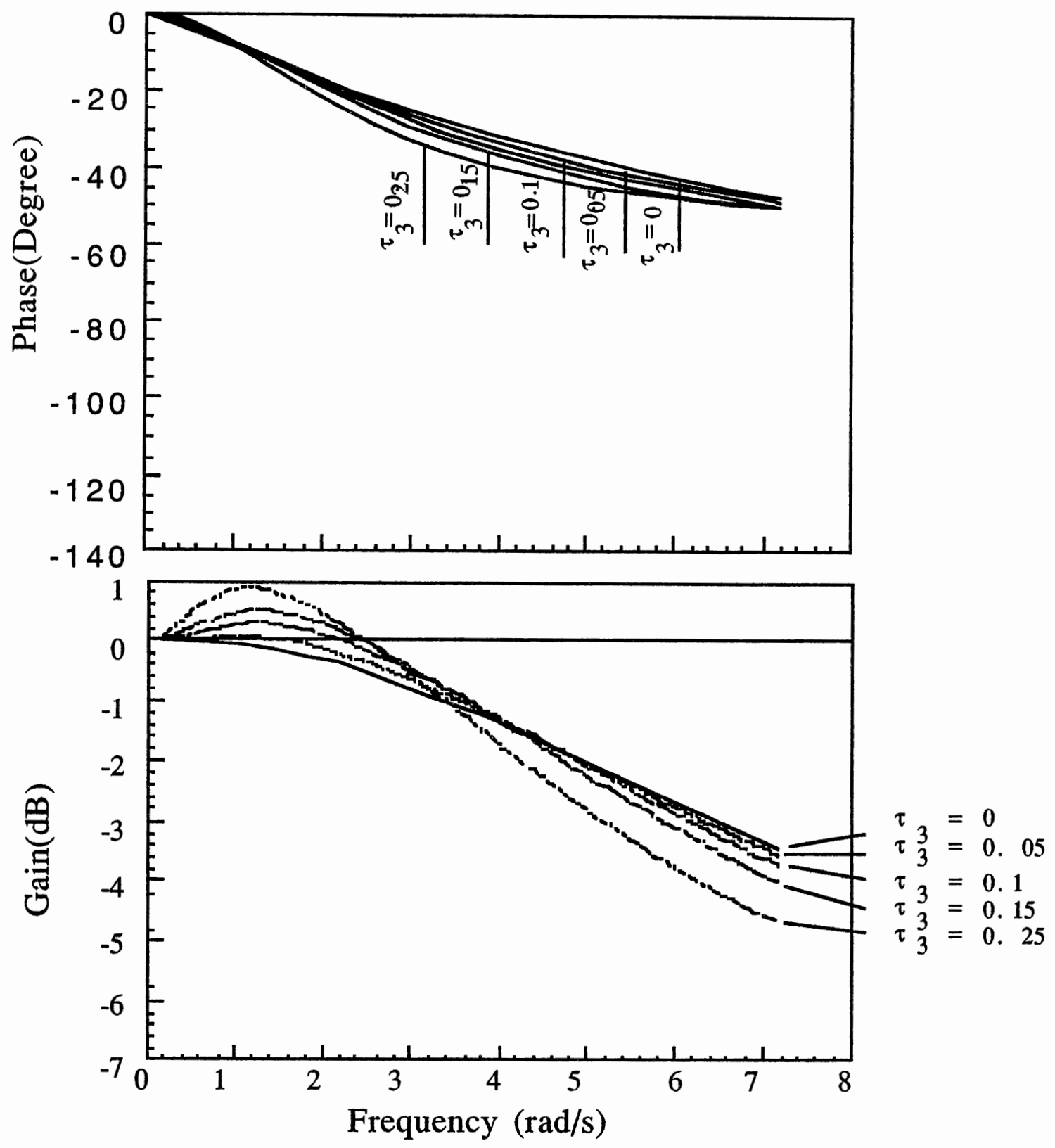

Figure 6.13a. Phase And Magnitude of $\mathrm{H}_{2}(\mathrm{~s})$ (delayed error is minimized) 

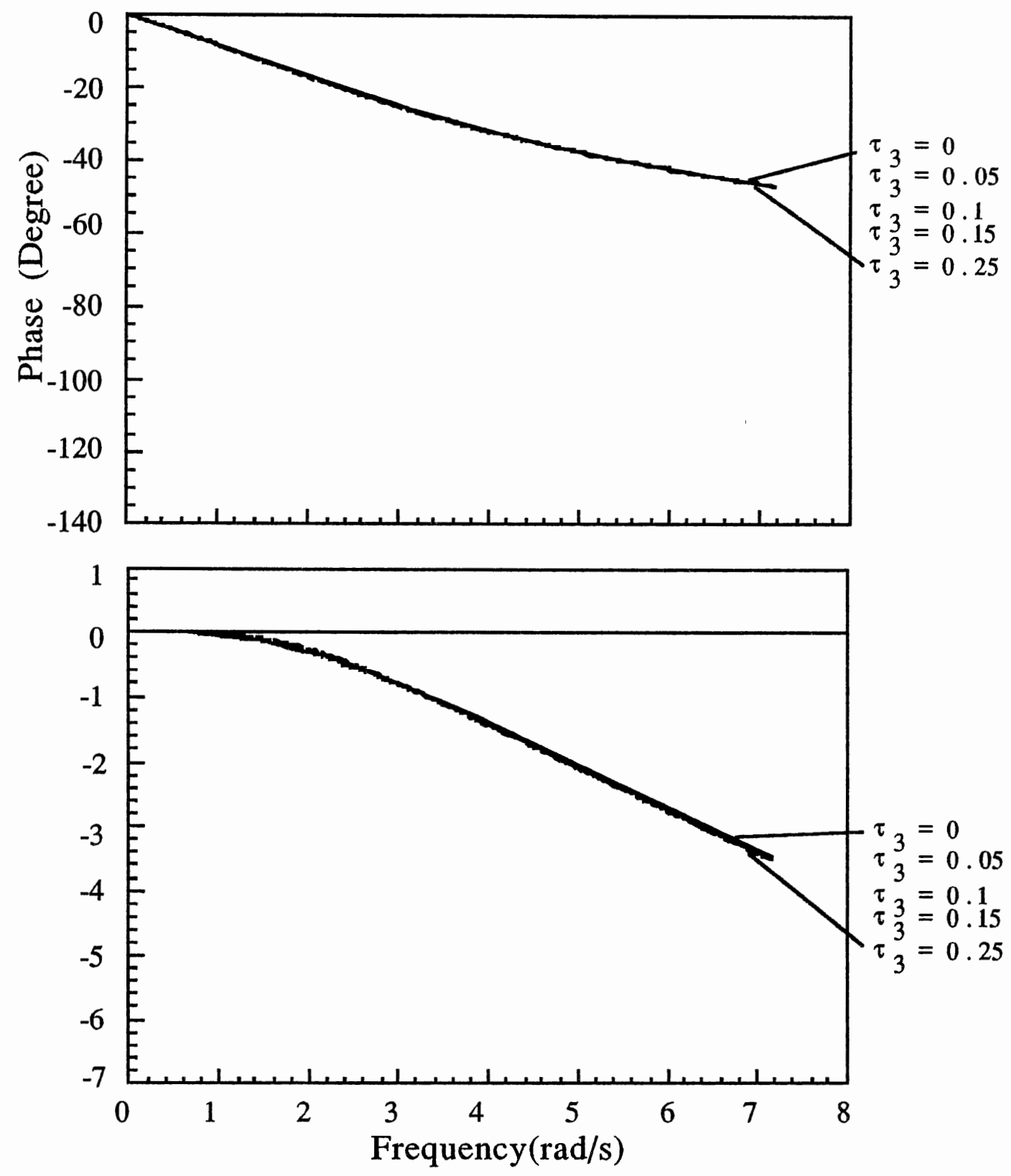

Figure 6.13b. Phase And Magnitude Of $\mathrm{H}_{2}(\mathrm{~s})$ (actual error is minimized) 
To summarize, this section considered a simple optimal tracking problem with an inter-simulator time delay, $\tau_{3}$. The behavior of the actual and delayed errors have been analyzed as a function of $\tau_{3}$. We observed that the actual error and delayed error show two exactly opposite trends (which have been seen in the formation flight task) as a function of $\tau_{3}$ As shown in chapter 5, the formation flight task is itself a special tracking problem (in which the reference model and the plant model experience the same disturbance) in a complex form. The opposite trends mentioned above are related to the nature of the general tracking problem.

\subsection{Simple Tracking With OCM}

The two unexpected properties of the rms error curves of the networked simulator formation flight tasks, non-monotonic and opposite trends of actual and delayed error, have been discussed in sections 6.2 and 6.3 , respectively. These sections used simple optimal tracking problems to illustrate the non-monotonic and opposite behavior of errors.

This section considers a simple tracking problem with an OCM depicted in Figure 6.14. As indicated in chapter V, this problem may represent a simple form of the dual OCM. The upper block diagram represents the reference model with external disturbance $w$ and the lower path contains the plant model and the OCM structure. The purpose of this section is to illustrate the effect of internal delay, $\tau_{2}$ on OCM model and to describe the effect of the OCM, as a special class of optimal filter and controller, on overall system performance. 
As discussed in chapter IV, the knowledge of the human parameters $\tau_{2}, \tau_{\mathrm{n}}, \mathrm{v}_{\mathrm{y}}$ and $\mathrm{v}_{\mathrm{u}}$ are necessary in order to implement the OCM. Here, the values of internal time-delay, $\tau_{2}$ and motor lag, $\tau_{n}$ are 0.15 and 0.1 second respectively. The value of control rate, $\mathrm{g}=0.003275$ was adjusted in order to have the required $\tau_{\mathrm{n}}=0.1 \mathrm{~s}$. The arbitrary values of the intensities for the observation noise, $\mathrm{v}_{\mathrm{y}}=0.1$, and motor noise, $\mathrm{v}_{\mathrm{u}}=0.1$, were chosen.

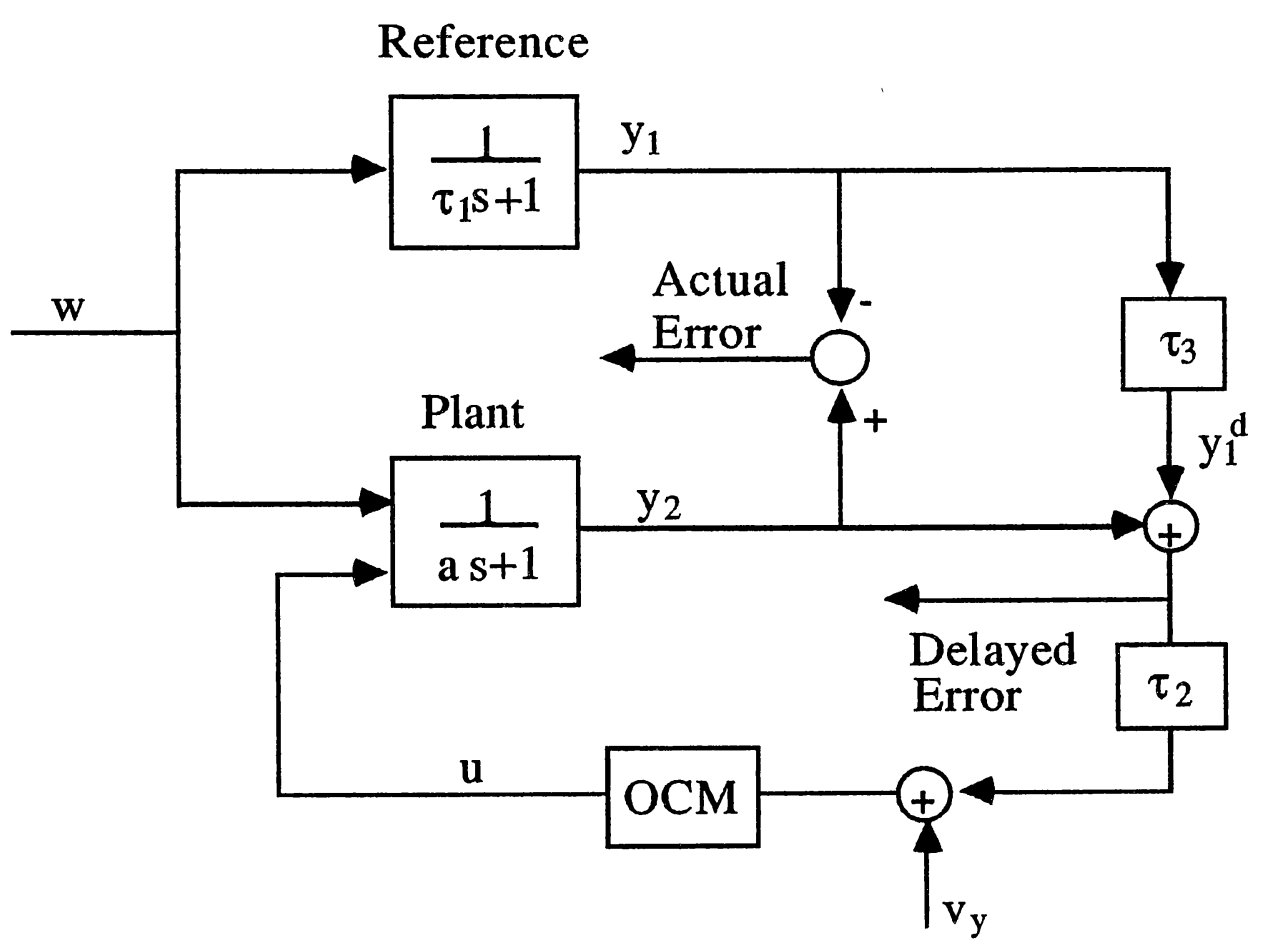

Figure 6.14. A simple Tracking Problem With OCM 
Figure 6.15 displays the optimal mean square errors versus $\tau_{1}$ for different values of the plant parameter $a$, and with intrasimulator delay $\tau_{3}=0$. The error as a function of $\tau_{1}$ drops initially and reaches its minimum at $\tau_{1}=a$ before increasing.

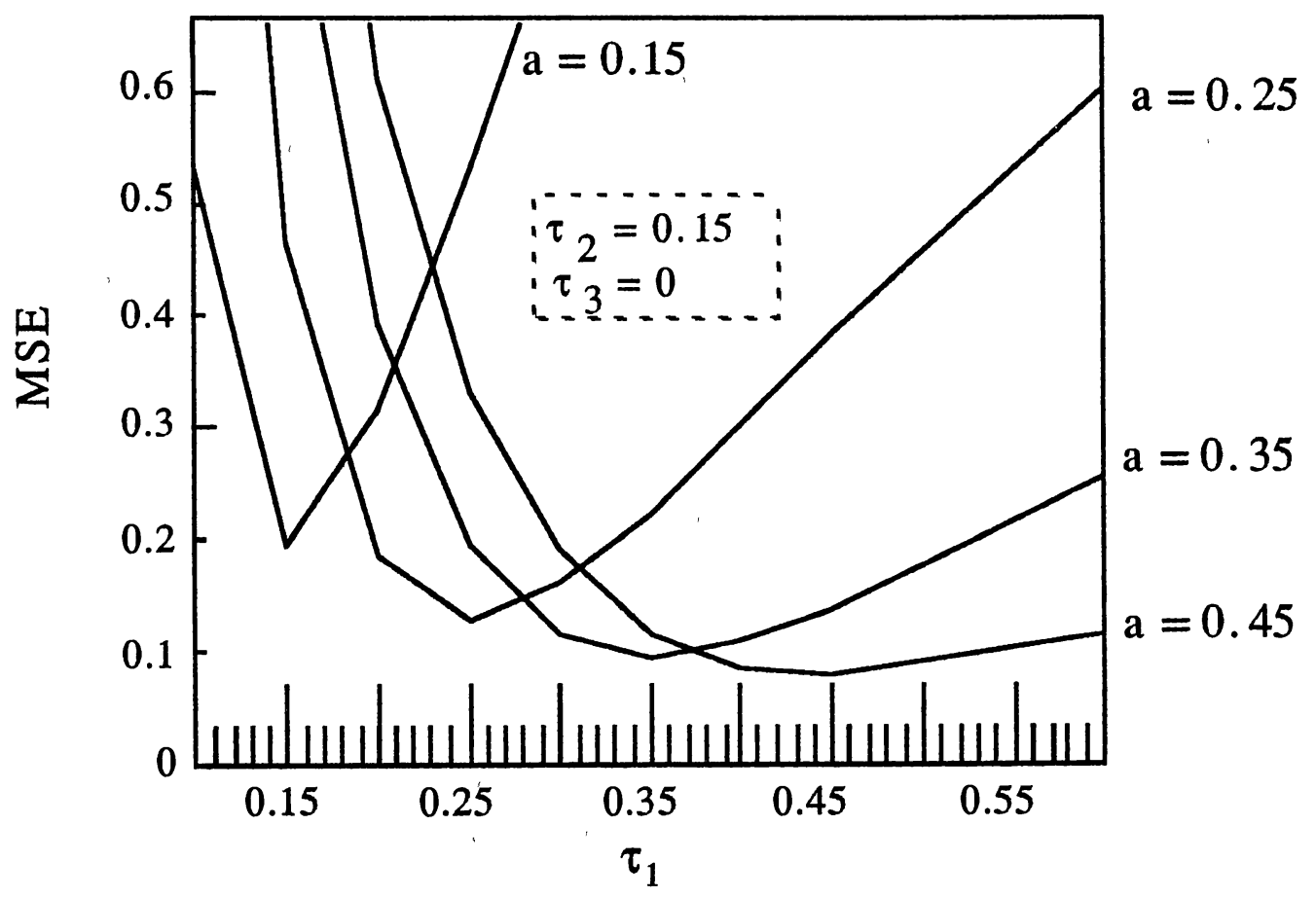

Figure 6.15. The Effect Of Plant Parameter "a" on MSE

By Comparing Figure 6.15 with Figure 6.9 (simple optimal control problem), we can see the effect of internal delay $\tau_{2}$ and motor noise $v_{u}$ (human operator limitations) on MSE. Here, we cannot have a zero error for the case in which $\tau_{1}=a$ (perfect match) because of human operator's limitations. The gain and phase plots of $\mathrm{H}_{2}(\mathrm{~s})$ 
are depicted in Figure 6.16 for different values of $\tau_{1}$. As seen, the optimal controller attempts to make $\mathrm{H}_{2}(\mathrm{~s})$ match $\mathrm{H}_{1}(\mathrm{~s})$. As $\tau_{1}$ increases, the gain of $\mathrm{H}_{2}(\mathrm{~s})$ increases and phase of $\mathrm{H}_{2}(\mathrm{~s})$ decreases. The frequency plots $\mathrm{H}_{2}(\mathrm{~s})$ match those of $\mathrm{H}_{1}(\mathrm{~s})$ best when $\tau_{1}=\mathrm{a}=0.15$.
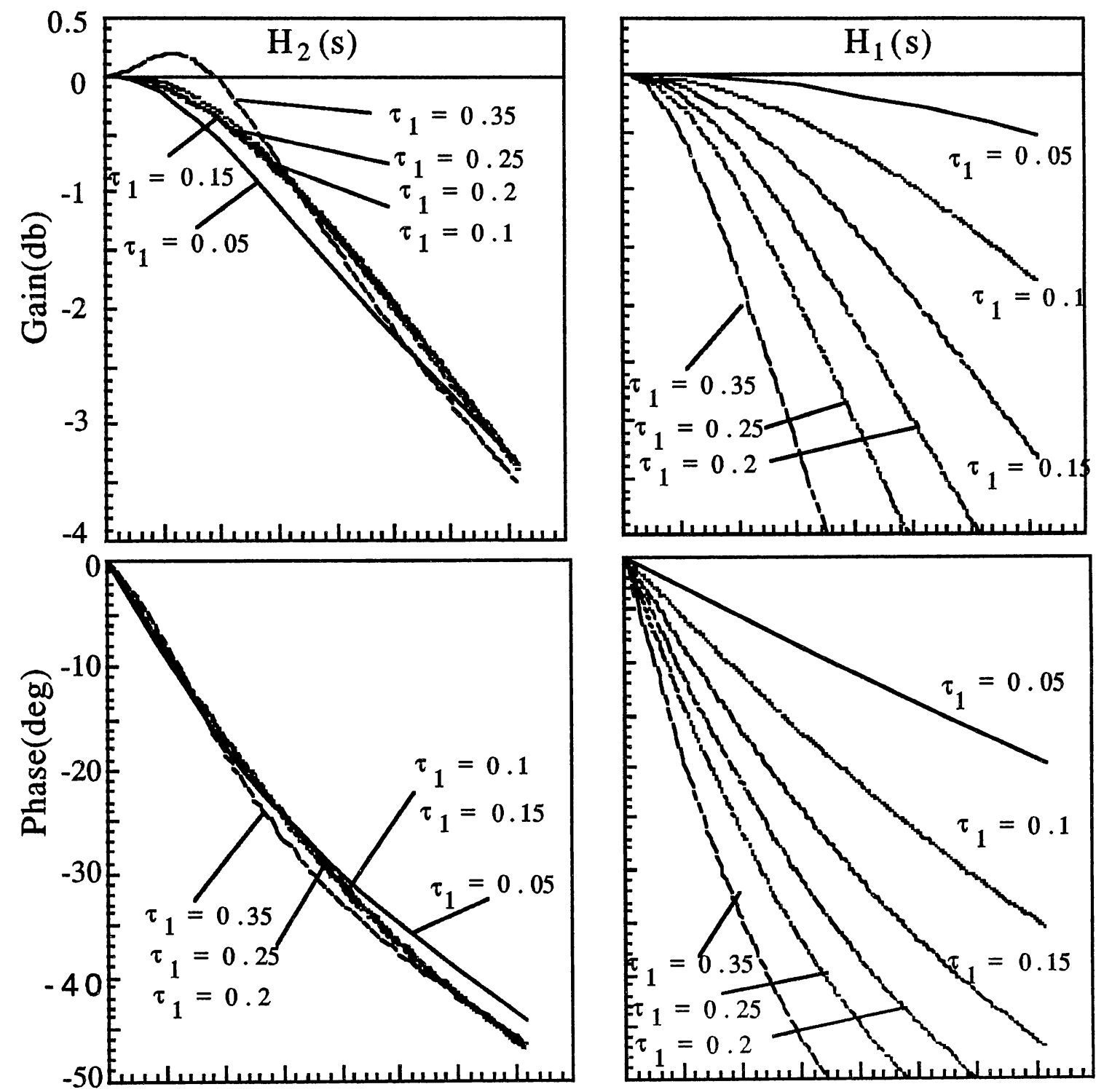

Figure 6.16. Frequency Plots of $\mathrm{H}_{2}(\mathrm{~s}) \& \mathrm{H}_{1}(\mathrm{~s})$ vs. $\tau_{1}$

$$
\left(\mathrm{a}=\tau_{2}=0.15, \tau_{3}=0\right)
$$




\subsubsection{Effect Of $\tau_{2}$ On OCM Response}

The effect of internal delay $\tau_{2}$ on human operator frequency response is best illustrated in Figure 6.17. Bigger delay $\tau_{2}$ yields smaller gain and bigger phase shift (especially at lower frequencies). It means that the human operator tries to deal with the increased system lags by adapting his behavior in order to make the overall system stable.

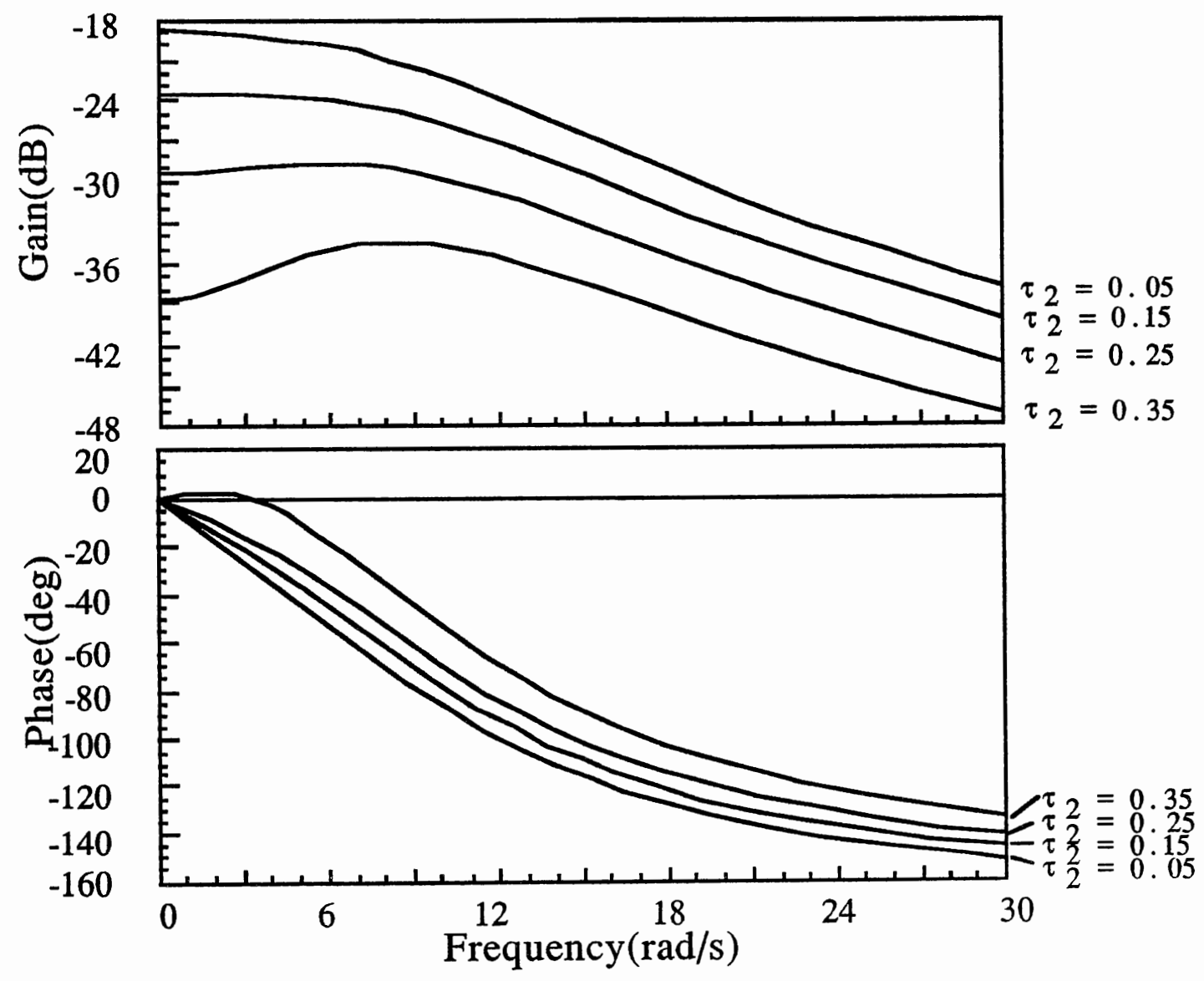

Figure 6.17. Frequency Plots of OCM $\left(a=\tau_{1}=0.15, \tau_{3}=0\right)$ 
In other words, when $\tau_{2}$, the man-machine phase lag, is increased, the human operator reduces his gain in order to guarantee closed-loop stability (The feedback controller becomes sluggish, and therefore the controller input becomes less significant). He also produces more phase lead in order to compensate for the lag introduced by $\tau_{2}$.

As $\tau_{1}$ increases, the gain of $\mathrm{H}_{2}(\mathrm{~s})$ increases and the phase of $\mathrm{H}_{2}(\mathrm{~s})$ decreases. The frequency plots $\mathrm{H}_{2}(\mathrm{~s})$ match those of $\mathrm{H}_{1}(\mathrm{~s})$ best when $\tau_{1}=\mathrm{a}=0.15$.

\subsubsection{Effect Of $\tau_{2}$ on MSE}

Figure 6.18 shows the effects of $\tau_{2}$ on mean square tracking error. The errors show different trends. $D_{d}$ decreases initially and reaches its minimum at $\tau_{2}=\tau_{3}$, before increasing. $A_{a}$ increases monotonically with $\tau_{2} \cdot \mathrm{D}_{\mathrm{a}}$ and $\mathrm{A}_{\mathrm{d}}$ are monotonically decreasing as $\tau_{2}$ increases.

The reason why $D_{d}$ behaves non-monotonically can be stated as follows. Figure 6.19 represents a reconfiguration of the blockdiagram of Figure 6.14 when the delayed error is minimized. As indicated in Figure 6.19, for the case in which the inter-simulator delay $\tau_{3}$ is equal to internal delay $\tau_{2}$, we will return to the case in which the delay $\tau_{3}=0$ (see Figure 6.14). This case has been discussed earlier in this section (see Figure 6.15). For $\tau_{1}=\mathbf{a}$, as $\tau_{2}$ increases, the control input $\mathbf{u}$ becomes less significant, and both the reference model and the plant respond to the same external input, w. 

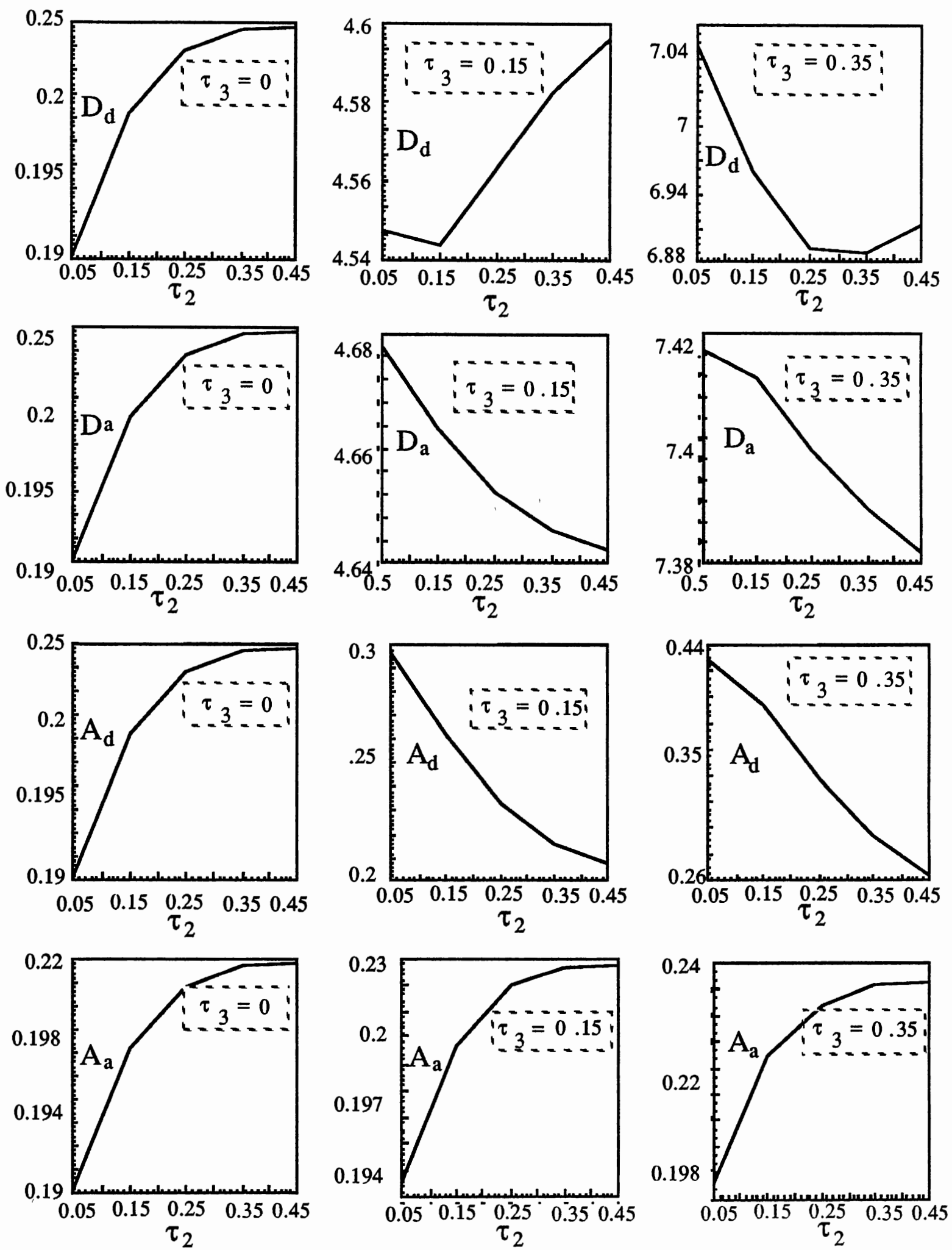

Figure 6.18. The Effect of Internal Delay $\tau_{2}$ on MSE

$$
\left(\mathrm{a}=\tau_{1}=0.15\right)
$$


Therefore, $D_{d}$ should drop initially when $\tau_{2}<\tau_{3}$ and reach a minimum at $\tau_{2}=\tau_{3}$ before increasing. One can use the same argument and explain why the other trends occur.

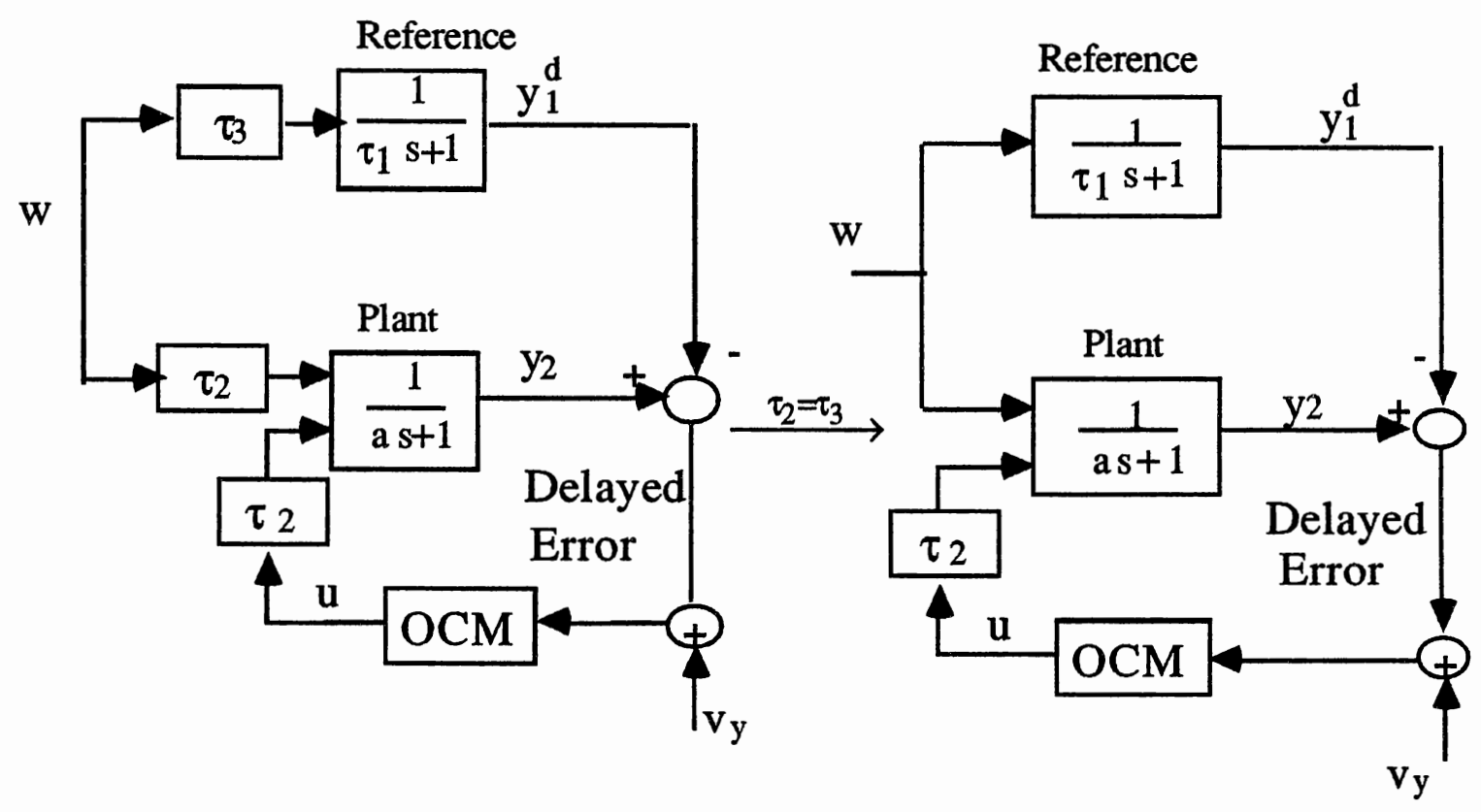

Figure 6.19. Reconfiguration of Figure 6.14 (when delayed error is minimized)

\subsection{Lower Order Dual OCM Results}

Section 6.1 considered the effects of intra-simulator timedelay, $\tau_{3}$, on pilot performance in a formation flight task, and it has been concluded that the minimum (maximum) value of mean square tracking error as a function of $\tau_{3}$, increases (decreases) with increasing $\tau_{2}$ and decreases (increases) with decreasing $\tau_{1}$. The 
OCMs in the formation flight task controlled vehicles which were modeled by fourth order dynamic systems.

Knowing that the behavior of the human operator is strongly dependent on the dynamics of the vehicles which he manipulates, this section considers dual OCMs with lower order vehicle dynamics, in order to check further the behavior of rms tracking errors. Sections 6.5.1 and 6.5.2 summarize the analytical results obtained from a dual OCM with first order and second order vehicle dynamics, respectively.

For the experimental cases in this section, the intrasimulator delay $\tau_{3}$ was varied over the range 0.001 to 0.6 seconds and internal delays $\tau_{1}$ and $\tau_{2}$ were varied over the range $0.05-0.55$ seconds. For both OCMs, the normalized observation noises on error and error rate are adjusted to $-20 \mathrm{~dB}$ by appropriate choices of observation noises $\mathrm{V}_{\mathrm{y}_{1}}$ and $\mathrm{V}_{\mathrm{y}_{2}}$. The normalized motor noises are adjusted to $-25 \mathrm{~dB}$ by selecting the right values for motor noise intensities $V_{u_{1}}$ and $V_{u_{2}}$. The nominal values for motor lags $\tau_{n_{1}}=\tau_{n_{2}}=0.1 \mathrm{~s}$ are chosen by adjusting control rates $g_{1}$ and $g_{2}$.

\subsubsection{Analytical Results of a}

First Order Dual OCM

The dynamics of both vehicles in dual OCM (see Figure 6.1) are governed by the following first order dynamical equations

$$
\begin{aligned}
& \dot{x}(t)=-x(t)+u(t)+w(t) \\
& y(t)=x(t),
\end{aligned}
$$


with the following block-diagram.

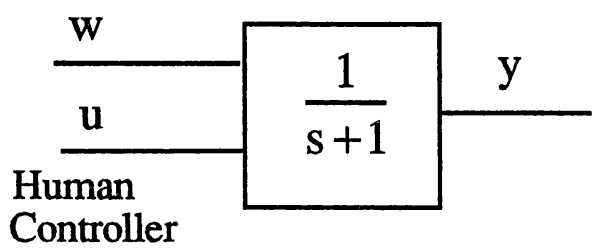

Figure 6.20 illustrates the effects of time-delays on the mean square tracking errors. As a function of intra-simulator delay $\tau_{3}$, the delayed error increases monotonically and the actual error drops initially to reach its minimum before increasing. As shown in Figure 6.20 , for a fixed second internal delay $\tau_{2}$, as the first internal delay $\tau_{1}$ increases the actual error decreases, and its minimum occurs at a smaller $\tau_{3}$. If we hold $\tau_{1}$ fixed and let $\tau_{2}$ increase, the actual error is increased and will reach its minimum later (at a larger $\tau_{3}$ ).

In summary, we saw that the delayed error and actual error, for a simple first order dual OCM, show two opposite behaviors, which are exactly the same trends which have been observed for a simple optimal tracking problem discussed in section 6.3.

\subsubsection{Second Order Dual OCM}

This section summarizes the effects of time-delays on mean square tracking errors for a dual OCM whose vehicle dynamics are both defined by the following second order system.

$$
\begin{aligned}
& \underline{\dot{x}}(t)=\left[\begin{array}{cc}
0 & 1 \\
-1 & -2
\end{array}\right] \underline{x}+\left[\begin{array}{l}
0 \\
1
\end{array}\right] u(t)+\left[\begin{array}{l}
0 \\
1
\end{array}\right] w(t) \\
& \underline{y}=\left[\begin{array}{ll}
1 & 0 \\
0 & 1
\end{array}\right] \underline{x} .
\end{aligned}
$$



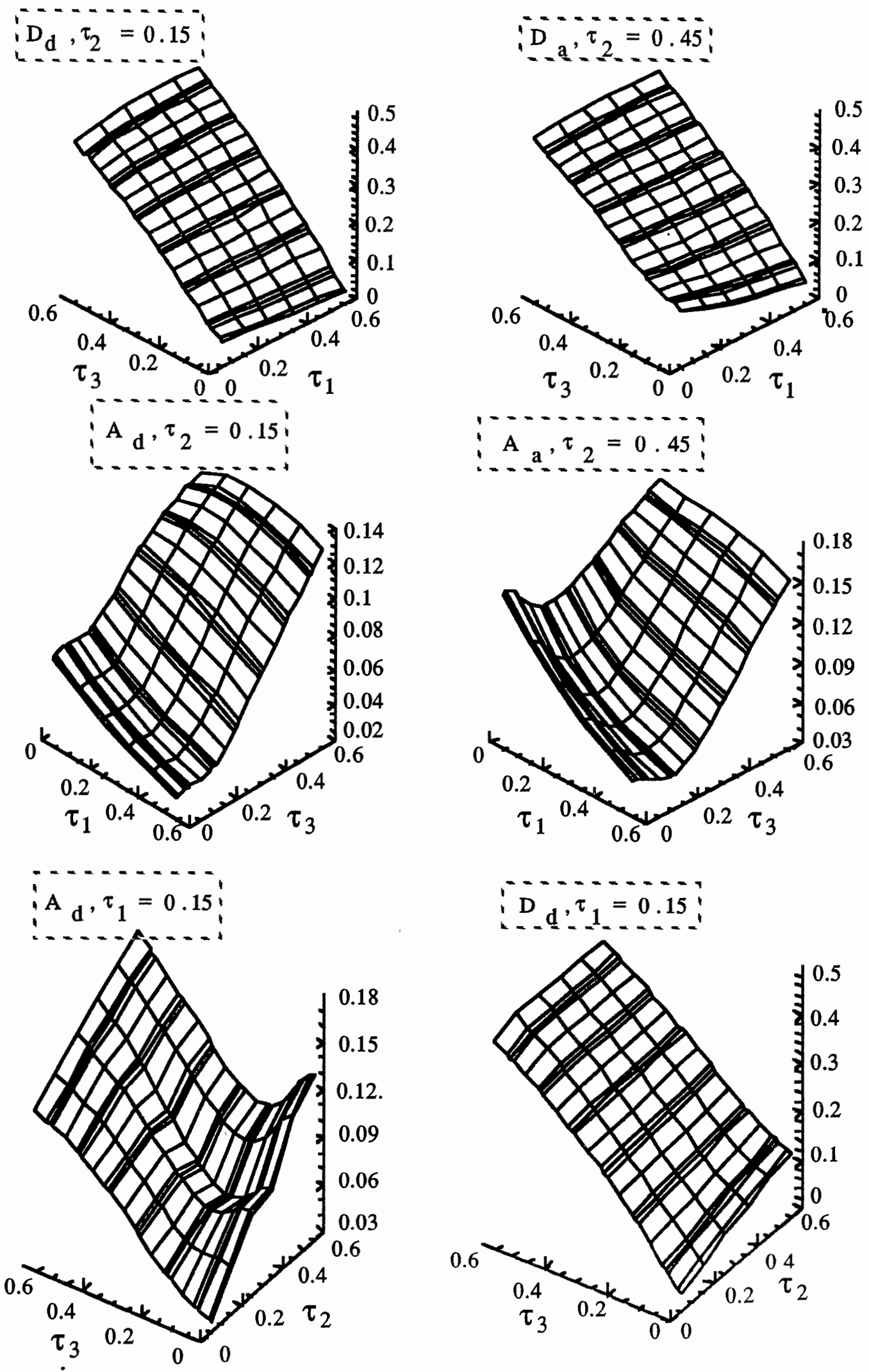

Figure 6.20. Effects of Delays on MSE in a First Order Dual OCM 
Figures 6.21 and 6.22 illustrate the effects of time-delays on mean square tracking error for this second order dual OCM. As seen, as afunction of inter-simulator delay $\tau_{3}$, the delayed error drops initially and reaches a minimum before increasing and the actual error goes up initially and will reach its maximum before decreasing.
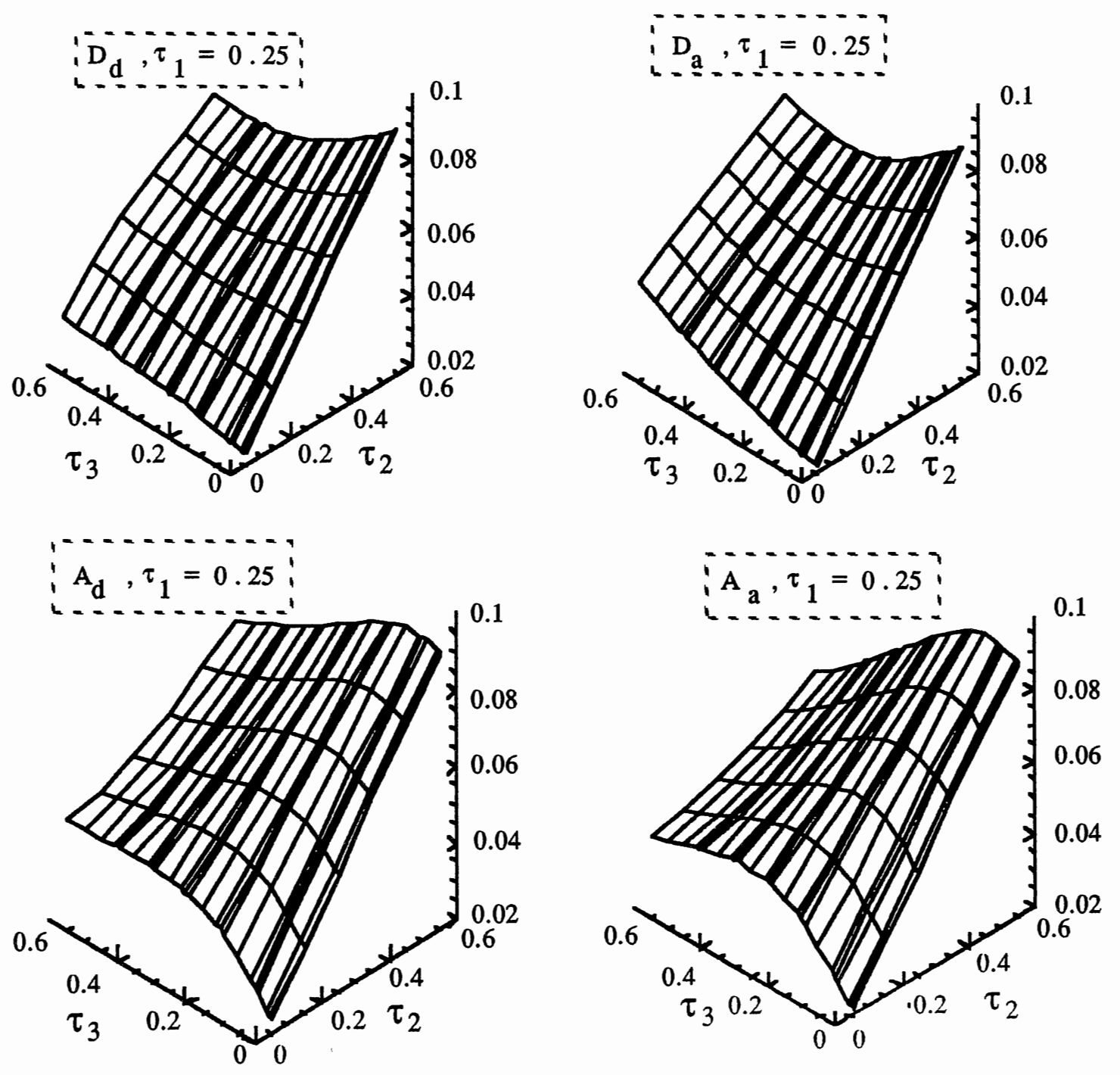

Figure 6.21. Effect of Delay $\tau_{2}$ on MSE for a Second Order Dual OCM 
The stationary points are varied as a function of internal delays. As indicated in Figure 6.21, If we hold the first internal delay $\tau_{1}$ fixed and increase the second internal delay $\tau_{2}$, the mean square errors are increased, and the delayed error will reach its minimum later (at alarger $\tau_{3}$ ), whereas the actual error reaches its maximum faster (at a smaller $\tau_{3}$ ).
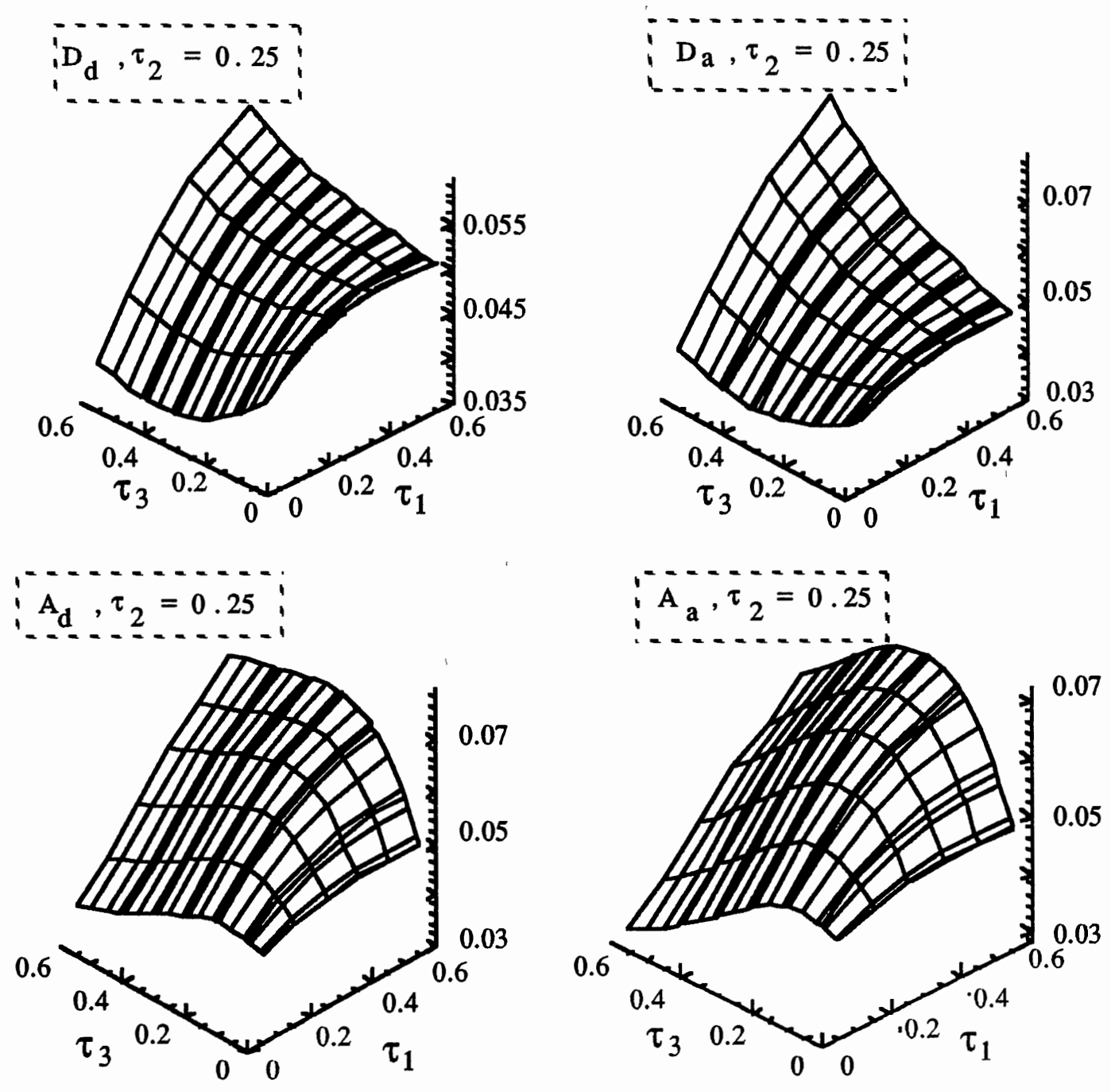

Figure 6.22. Effect of Delay $\tau_{1}$ on MSE for a Second Order Dual OCM 
In short, for second order dual OCMs we have observed that the mean square tracking errors possess exactly the same trends which have been seen in formation flight task experiments. These trends have been discussed in section 6.1.

\subsection{Summary}

In this chapter we investigated the effects of system delays on human performance in dual OCM problems. The analytical results obtained from a formation flight task showed that the mean square tracking errors (perceived and actual errors), as a function of intersimulator delay, are non-monotonic, and they behave in two opposite ways. The mean square perceived error drops initially to reach a minimum before increasing, whereas the mean square actual error increases initially and will reach a maximum before decreasing.

These trends have been investigated in sections 6.2 and 6.3 by considering some simple optimal tracking problems, and it has been concluded that the non-monotonic and opposite behaviors of errors are related to the nature of the general tracking problem, since the formation flight task, in which the first pilot tries to mimic the second pilot, is itself a tracking problem in a complex form.

The effect of internal delay on the OCM, as a special class of optimal filter and controller, and on mean square tracking error, have been discussed in section 6.4 by considering a simple tracking problem with an OCM used as an optimal controller feedback. It has been shown that by increasing internal delay, the human operator 
reduces his gain and produces more phase lead in order to guarantee the stability of the closed loop system.

By considering the lower order dual OCMs in section 6.5 , we observed that although the behavior of human operator is strongly affected by the complexity of the vehicles which he manipulates, the overall closed-loop performance of a dual OCM problem, where the first OCM attempts to follow the second OCM, is governed by the general nature of the stochastic tracking problem. 
CHAPTER VII

\section{AN INTRODUCTION TO THE AIR-TO-AIR COMBAT TASK}

\subsection{Introduction}

So far it has been shown that we can use the optimal control model methodology to analyze the adverse effects of the communication delay in a formation flight task, a special class of problem. The formation flight task is formulated as a dual OCM in which the first OCM does not interact with the second OCM.

The formation flight task, which was analyzed in this report, is only one task in which inter-simulator time delay may cause problems. The air-to-air combat task, for example, could be even more sensitive to the effects of delays between the simulators. The next phase of this research should concentrate on this more challenging problem.

The difficulty in analyzing the air-to-air combat task stems from the fact that the OCM assumes that each pilot has an accurate internal model of the target. In this case the target is another aircraft, with a pilot who is also tracking the first aircraft.

Hence, the air-to-air combat task is basically different from the formation flight task discussed in previous chapters Consequently, the 
dual OCM formulated in chapter $\mathrm{V}$ can not be applied to an air-to-air combat problem.

The "game of combat", which includes air-to-air combat, is referred to as an encounter between hostile players, each of whom attempts to capture (destroy) his opponent, while ensuring his own survival. The combat game becomes a one-target, pursuer-evader game if one of the players has no offensive capabilities so that he can never destroy his opponent. The offensive player is called the pursuer, and his opponent (the defensive player) is called the evader. The pursuer chooses policies to minimize the time of the capture of his opponent, and the evader tries to prevent the capture or to maximize the time of capture.

The general mathematical approach for formulating and solving pursuit-evasion problems is the theory of differential games due to Isaacs, 1954 [81]. Since that time till now, much work has been done on games of pursuit-evasion [82-107].

This chapter, which considers the feasibility of developing an OCM air-to-air combat model is organized as follows. Sections 7.2 through 7.4 present a review of the literature on differential game theory and introduce pursuit-evasion problems. In section 7.5, we apply the methods reviewed in the previous sections to an example of a stochastic pursuit-evasion game (classical interception problem). Section 7.6 investigates the feasibility of applying differential games theory to modeling of an air-to-air combat task by considering the possibility of combining the optimal control model and differential games for a simple task. Finally, section 7.7 concludes this chapter. 


\subsection{Differential Games}

The theory of differential games was invented by $R$. Isaacs in 1954 [81]. His study is basically an extension of pursuit problems in which the target can control his motion (maneuverable target) and his solution is rooted in both game theory and modern control theory [90]. We proceed the mathematical formulation of the differential game theory in section 7.2.2 after reviewing some concepts of game theory, which are necessary in order to have a better understanding of the differential games.

\subsubsection{Game theory}

The theory of games, which was first developed by Jon Von Neuman in 1920's, may be viewed as a decision-making processe involving two players with the conflicting optimal strategies [8083,116]. A strategy is defined as a set of decisions which provide an alternative for the player in order to take an action. The players take the strategies based on some certain available information, including the state of the game and the rules of the game. These rules determine the objectives of the game, the end of the game, and present the admissible set of strategies of each player of the game.

The game is called zero-sum if each player's loss is his opponent's gain. There are two basic premises of zero-sum two player deterministic games: (1) each player is trying to maximize his own gains, or to minimize his own losses, (2) both players are able enough to evaluate accurately the payoffs associated with both opponent's alternative strategies (perfect information game). The 
payoff is a numerical measure which determines the value of a game as well as the values of strategies; it is expressed in terms of the strategies of both players.

The payoffs associated with the players' alternative strategies are regularly expressed in the form of a payoff matrix. Thus the $n \times m$ payoff matrix means that the first player, $u$, can choose a selection of pure strategies associated with the m payoff rows, while the other player, $v$, may choose a selection of strategies associated with the $n$ payoff columns. The payoff will be the number at the joint point of their two choices, for example if the player $u$ chooses the ith row and the player $v$ chooses the column $j$, the payoff is the $i j$-th element of the payoff matrix.

In order to introduce conflict, we assume that the player $v$ tries to maximize the payoff by choosing his appropriate choice of strategy, while the player $u$ attempts to minimize the same payoff of the game by his selection of a strategy. Since it is a perfect information game in a sense that $u$ and $v$ know their objectives, $v$ strives for the maximum of the minima (maximin), while $u$ strives for the minimum of the maxima (minimax.)

The game has a saddle point if the minimax equals the maximin. The solution to a game means a statement of the probabilities which each player has to assign to each of his strategies.

Given a game with a saddle point, finding the solution of the game is simply finding the pure single strategy for each player will result in the maximin equalling the minimax. Given a game with no saddle point, a mixed strategy (i.e., a combination of pure strategies with a given frequency) will yield the same stability (i.e., u's minimax 
equates the v's maximin) as in the games with a saddle point*. This payoff is called the value of game which indicates the expected value of v's gains and u's losses.

If the choices of $u$ and $v$ are continuous, the discrete payoff matrix is replaced by a continuous payoff function expressed explicitly in terms of the strategies of $u$ and $v, L(u, v)$, where $L$ denotes the payoff of the game. The main concern in game theory is to find a pair of strategies $u^{0}$ and $v^{0}$ such that

$$
\mathrm{J}\left(\mathrm{u}^{\mathrm{o}}, \mathrm{v}\right) \leq \mathrm{J}\left(\mathrm{u}^{\mathrm{o}}, \mathrm{v}^{\mathrm{o}}\right) \leq \mathrm{J}\left(\mathrm{u}, \mathrm{v}^{\mathrm{o}}\right)
$$

with $u \in U$ and $v \in V$, where $U$ and $V$ are the sets of all admissible strategies for the minimizing and maximizing players, respectively.

The pair $\left(\mathbf{u}^{0}, v^{0}\right)$ is called a saddle point of the game and $J\left(u^{0}, v^{0}\right)$, which is the payoff evaluated at the saddle point, is called the value of the game. A game theoretic saddle point exits if the following conditions are satisfied [80]

$$
\begin{aligned}
& \frac{\partial \mathrm{L}(\mathrm{u}, \mathrm{v})}{\partial(\mathrm{u})}=\frac{\partial \mathrm{L}(\mathrm{u}, \mathrm{v})}{\partial(\mathrm{v})}=0 \\
& \frac{\partial^{2} \mathrm{~L}(\mathrm{u}, \mathrm{v})}{\partial\left(\mathrm{u}^{2}\right)} \geq 0, \quad \frac{\partial^{2} \mathrm{~L}(\mathrm{u}, \mathrm{v})}{\partial\left(\mathrm{v}^{2}\right)} \leq 0
\end{aligned}
$$

and

$$
\frac{\partial^{2} \mathrm{~L}(\mathrm{u}, \mathrm{v})}{\partial\left(\mathrm{u}^{2}\right)}>0, \frac{\partial^{2} \mathrm{~L}(\mathrm{u}, \mathrm{v})}{\partial\left(\mathrm{v}^{2}\right)}<0
$$

\footnotetext{
* This is the result of minimax theorem whichwas proved in 1928 by John von Neuman. This theorem says that by introducing mixed strategies we are able to find a solution for any two person zero-sum game.
} 
where the equations (7.2) and the equation (7.3) are respectively the necessary and sufficient conditions for $u^{0}$ and $v^{0}$. The equation (7.1) is equivalent to the following minimax equation :

$$
\begin{aligned}
& \mathrm{J}\left(\mathrm{u}^{\mathrm{o}}, \mathrm{v}^{\mathrm{o}}\right)=\underset{\mathrm{u} \in \mathrm{U}}{\operatorname{Min}} \mathrm{J}\left(\mathrm{u}, \mathrm{v}^{\mathrm{o}}\right)=\underset{\mathrm{v} \in \mathrm{V}}{\operatorname{Max}} \mathrm{J}\left(\mathbf{u}^{\mathrm{o}}, \mathrm{v}\right) \\
& =\operatorname{Min}_{\mathbf{u} \in \mathrm{U}} \underset{\mathbf{v a x} \in \mathrm{V}}{\operatorname{Max}} \mathrm{J}(\mathbf{u}, \mathbf{v}) \\
& =\underset{\mathbf{v} \in \mathrm{V}}{\operatorname{Max}} \operatorname{Min}_{\mathbf{u} \in \mathrm{U}} \mathrm{J}(\mathrm{u}, \mathrm{v})
\end{aligned}
$$

Equation (7.4) is obtained based on the assumption that each player takes his optimal strategy while assuming that his opponents plays optimally.

\subsubsection{Differential Games}

A mathematical definition of a general differential game may be stated as ${ }^{\#}$ : determine a saddle-point pair of pure strategies $\underline{u}^{\circ}$ and $\underline{v}^{0}$ of the continuous payoff index

$$
J\left(t_{0}, \underline{x}_{0} ; \underline{u}, \underline{v}\right)=\Gamma\left(\underline{x}\left(t_{f}\right), t_{f}\right)+\int_{t_{0}}^{t_{f}} L(\underline{x}(t), \underline{u}(t), \underline{v}(t)) d t
$$

subject to the state constraints

$$
\begin{aligned}
& \underline{\dot{x}}(t)=f(\underline{x}(t), \underline{u}(t), \underline{v}(t)), \quad \underline{x}\left(t_{0}\right)=\underline{x}_{0}, \\
& \psi\left(\underline{x}\left(t_{f}\right)\right)=0 .
\end{aligned}
$$

\# A rigorous formulation of differential games termed as quantitative games is given in Chapters I-V of Ref. 113. 
One may refer to the differential game as a generalized game theory problem. For example, it becomes a game theory problem discussed in the previous section if the state $\underline{x}$ can be solved in terms of control inputs $\underline{\mathbf{u}}$ and $\underline{\mathbf{v}}$. The state constraints given above define the rules of the game, i.e., the start, the progress and the end of the game. The differential game defined above is called a zero-sum, two person game, and its solution has been fully discussed in the literature [81,84-88].

The differential game problem as a 'two-sided' optimal control problem [80] becomes an optimal control problem if both players minimize (maximize) the payoff index $J$ or if one of the players does not play (inactive). Ref. [108] formulates a particular case of differential game which can be redeuced and considred as a statndard optimal control processes. Conversely, the optimal control problems can be generalized to differential games by choosing an opponent (max-min control problems or games against nature $[109,116])$. That is why the strategies are the control inputs and are defined in the form of linear state feedback control laws.

As we mentioned at the beginning of this chapter, the differential games which date back to the 1950's concerned the study of the combat problems [81]. These kind of problems can be formulated as pursuit-evasion games. The next two sections consider deterministic and stochastic linear pursuit-evasion games, respectively. 


\subsection{Linear Deterministic Pursuit- \\ Evasion Game [80,82]}

This section considers a class of differential games which is interpreted as a pursuit-evasion problem. This problem is formulated as follows. Assume that the dynamics of pursuer and evader are defined accurately by the following state equations,

$$
\begin{array}{lll}
\underline{\dot{x}}_{p}(t)=A_{p} \underline{x}_{p}(t)+B_{p} \underline{u}(t) & , & \underline{x}_{p}\left(t_{0}\right)=\underline{x}_{p_{0}} \\
\underline{\dot{x}}_{e}(t)=A_{e} \underline{x}_{e}(t)+B_{e} \underline{v}(t) \quad, & \underline{x}_{e}\left(t_{0}\right)=\underline{x}_{e} 0
\end{array}
$$

where subscripts $p$ and e stand for pursuer and evader, respectively. It is assumed that $\underline{x}_{p}$ and $\underline{x}_{e}$ are, respectively $n_{p}$-dimensional and $n_{e}$ dimensional state vector, and the control vectors $\underline{\mathbf{u}}$ and $\underline{v}$ are of dimension $r_{p}$ and $r_{e}$, respectively. The matrices $A_{p}, A_{e}, B_{p}$, and $B_{e}$ have the appropriate dimensions. The pursuer manipulates the control input $\underline{\mathbf{u}}$ to capture the evader, while the latter tries to avoid capture by using the control input, $\underline{\mathbf{v}}$. These inputs are limited as:

$$
\begin{aligned}
& \int_{t_{0}}^{t_{f}} \underline{u^{\prime}}(t) R_{p} \underline{u}(t) d(t) \leq E_{p} \\
& \int_{t_{0}}^{t_{f}} \underline{v}^{\prime}(t) R_{e} \underline{v}(t) d(t) \leq E_{e}
\end{aligned}
$$

where $t_{f}$ is a fixed final time, $R_{p}$ and $R_{e}$ are positive definite, and $E_{p}$ and $E_{e}$ denote the maximum energy sources of pursuer and evader, respectively.

For such linear systems given in (7.5) \& (7.6), the following differential game is considered: 
Find a saddle point $\left(\underline{u}^{0}\left(t ; \underline{x}_{0}, t_{0}\right), \underline{\mathbf{v}}^{0}\left(t ; \underline{x}_{0}, t_{0}\right)\right)$, of the payoff index

$$
\begin{aligned}
J\left(\underline{u}, \underline{v} ; t_{0}, \underline{x}_{0}\right)= & \frac{c^{2}}{2}\left(C_{p} \underline{x}_{p}\left(t_{f}\right)-C_{e} \underline{x}_{e}\left(t_{f}\right)\right)^{\prime}\left(C_{p} \underline{x}_{p}\left(t_{f}\right)-C_{e} \underline{x}_{e}\left(t_{f}\right)\right)+ \\
& \frac{1}{2} \int_{t_{0}}^{t_{f}} \underline{u}^{\prime}(t) R_{p} \underline{u}(t) d t-\frac{1}{2} \int_{t_{0}}^{t_{f}} \underline{v}^{\prime}(t) R_{e} \underline{v}(t) d t
\end{aligned}
$$

subject to the constraints given in Equations (7.5) and (7.6), where the matrices of $\mathrm{C}_{\mathrm{p}}$ and $\mathrm{C}_{e}$ yielding the interested states, are of dimensions $\mathbf{n} \times \mathbf{n}_{\mathrm{p}}$ and $\mathbf{n} \times \mathrm{n}_{\mathrm{e}}$, respectively. Both pursuer and evader are aware of the dynamics of both system (they know $\underline{x}_{p}$ and $\underline{x}_{e}$ at any time $t$ ); this is a game of perfect information. The positive parameter $c^{2}$ weights the final miss. The case in which $c^{2}$ is very large corresponds to the situation of the pursuer trying to capture the evader using some finite energy. The capture occurs if the difference between $C_{p} \underline{x}_{p}\left(t_{f}\right)$ and $\mathrm{C}_{\mathrm{e}} \underline{\mathrm{x}}_{\mathrm{e}}\left(\mathrm{t}_{\mathrm{f}}\right)$ becomes zero.

As indicated in (7.8), the objective of the game is that the pursuer attempts to minimize the final miss in order to intercept the evader at some fixed final time $t_{f}$, while the evader attempts to maximize the final miss; the miss is defined as a weighted quadratic index. The minus sign in the front of the third term in (7.8) is due to maximization of $J$ with respect to $\underline{v}$. 


\subsubsection{The Solution}

Let us define the predicted terminal miss vector

$$
\underline{\mathrm{x}}(\mathrm{t})=\left[\mathrm{C}_{\mathrm{p}} \Phi_{\mathrm{p}}\left(\mathrm{t}_{\mathrm{f}}, \mathrm{t}\right) \underline{\mathrm{x}}_{\mathrm{p}}(\mathrm{t})-\mathrm{C}_{\mathrm{e}} \Phi_{\mathrm{e}}\left(\mathrm{t}_{\mathrm{f}}, \mathrm{t}\right) \underline{\mathrm{x}}_{\mathrm{e}}(\mathrm{t})\right]
$$

where $\Phi_{p}\left(t_{f}, t\right)$ and $\Phi_{e}\left(t_{f}, t\right)$ are the transition matrices for the pursuer and the evader systems, respectively.

$$
\begin{aligned}
& \Phi_{p}\left(t_{f}, t\right)=e^{A_{p}\left(t_{f}-t\right)} \\
& \Phi_{e}\left(t_{f}, t\right)=e^{A_{e}\left(t_{f}-t\right)}
\end{aligned}
$$

From Equation (7.10), we can see that the state vector $\underline{x}(t)$ is actually the weighted difference between homogeneous solutions of (7.5) and (7.6), due to the initial conditions given at time t.

Differentiating both sides of (7.9) and knowing that

$$
\dot{\Phi}_{e}\left(t_{f}, t\right)=-A_{e} e^{A_{e}\left(t_{f}-t\right)} \text { and } \dot{\Phi}_{p}\left(t_{f}, t\right)=-A_{p} e^{A_{p}\left(t_{f}-t\right)} \text {, }
$$

we can write as:

$$
\begin{aligned}
\underline{\dot{x}}(t)= & C_{p}\left[\dot{\Phi}_{p} \underline{x}_{p}+\Phi_{p} \underline{\dot{x}}_{p}\right]-C_{e}\left[\dot{\Phi}_{e} \underline{x}_{e}+\Phi_{e} \underline{\dot{x}}_{e}\right] \Rightarrow \\
\underline{\dot{x}}(t)= & C_{p}\left[-A_{p} \Phi_{p} \underline{x}_{p}+\Phi_{p} A_{p} \underline{x}_{p}+\Phi_{p} B_{p} \underline{u}\right] \\
& -C_{e}\left[-A_{e} \Phi_{e} \underline{x}_{e}+\Phi_{e} A_{e} \underline{x}_{e}+\Phi_{e} B_{e} \underline{v}\right] \Rightarrow \\
\underline{\dot{x}}(t)= & C_{p} \Phi_{p}\left(t_{f}, t\right) B_{p} \underline{u}(t)-C_{e} \Phi_{e}\left(t_{f}, t\right) B_{e} \underline{v}(t) .
\end{aligned}
$$

To obtain (7.11), we used Equations (7.5), (7.6) and the fact that $\mathrm{e}^{\mathrm{K}(.)} \mathrm{K}=\mathrm{Ke} \mathrm{K}^{\mathrm{K}(.)}$. The index (7.8) can be written 


$$
\begin{aligned}
J\left(\underline{u}, \underline{v} ; t_{0}, \underline{x}_{0}\right) & =\frac{c^{2}}{2} \underline{x}^{\prime}\left(t_{f}\right) \underline{x}\left(t_{f}\right) \\
& +\frac{1}{2} \int_{t_{0}}^{t_{f}} \underline{u}^{\prime}(t) R_{p} \underline{u}(t) d t-\frac{1}{2} \int_{t_{0}}^{t_{f}} \underline{v}^{\prime}(t) R_{e} \underline{v}(t) d t
\end{aligned}
$$

where

$$
\underline{x}\left(t_{f}\right)=C_{p} \underline{x}_{p}\left(t_{f}\right)-C_{e} \underline{x}_{e}\left(t_{f}\right)
$$

Equation (7.13) is obtained by using the property $\Phi\left(t_{f}, t_{f}\right)=I$ in (7.9).

Thus, the problem of determining a saddle point of (7.12) subject to (7.11) is exactly equivalent to the original problem. In order to solve the above differential game, one may apply the standard variational procedures. By introducing the Hamiltonian

$$
\begin{aligned}
H(t, \underline{x}, \underline{u}, \underline{v}, \underline{\lambda}) & =\frac{1}{2} \underline{u}^{\prime}(t) R_{p} \underline{u}(t)-\frac{1}{2} \underline{v}^{\prime}(t) R_{e} \underline{v}(t) \\
& +\underline{\lambda}^{\prime}\left[P\left(t_{f}, t\right) \underline{u}(t)-E\left(t_{f}, t\right) \underline{v}(t)\right],
\end{aligned}
$$

the necessary conditions for a saddle point are

$$
\begin{aligned}
& H_{u}=\frac{\partial H}{\partial \underline{\mathrm{u}}}=R_{\mathrm{p}} \underline{\mathrm{u}}+\mathrm{P}^{\prime} \underline{\lambda}=0 \Rightarrow \underline{\mathrm{u}}^{0}=-\mathrm{R}_{\mathrm{p}}^{-1} \mathrm{P}^{\prime} \underline{\lambda} \\
& H_{v}=\frac{\partial H}{\partial \underline{\mathrm{v}}}=R_{\mathrm{e}} \underline{\mathrm{v}}+\mathrm{E}^{\prime} \underline{\lambda}=0 \Rightarrow \underline{\mathrm{v}}^{\mathrm{o}}=-\mathrm{R}_{\mathrm{e}}^{-1} \mathrm{E}^{\prime} \underline{\boldsymbol{\lambda}} \\
& \underline{\dot{\lambda}}(\mathrm{t})=-\mathrm{H}_{\mathrm{x}}=\frac{\partial \mathrm{H}}{\partial \underline{\mathrm{x}}}=0 \quad, \quad \underline{\lambda}\left(\mathrm{t}_{\mathrm{f}}\right)=\left.\frac{\mathrm{c}^{2}}{2} \frac{\partial\left(\underline{\mathrm{x}}^{\prime} \underline{\mathrm{x}}\right)}{\partial \underline{\mathrm{x}}}\right|_{\mathrm{t}_{\mathrm{f}}}=\mathrm{c}^{2} \underline{\mathrm{x}}\left(\mathrm{t}_{\mathrm{f}}\right),
\end{aligned}
$$

where

$$
P\left(t_{f}, t\right)=C_{p} \Phi_{p}\left(t_{f}, t\right) B_{p}
$$


and

$$
E\left(t_{f}, t\right)=C_{e} \Phi_{e}\left(t_{f}, t\right) B_{e}
$$

By substituting (7.15a) and (7.15b) into (7.11), we have

$$
\underline{\dot{x}}=\left(E\left(t_{f}, t\right) R_{e}^{-1} E^{\prime}\left(t_{f}, t\right)-P\left(t_{f}, t\right) R_{p}^{-1} P^{\prime}\left(t_{f}, t\right)\right) \underline{\lambda} .
$$

Equation (7.15c) solves for $\underline{\lambda}$,

$$
\underline{\lambda}(\mathrm{t})=\underline{\lambda}\left(\mathrm{t}_{\mathrm{f}}\right)=\mathrm{c}^{2} \underline{\mathrm{x}}\left(\mathrm{t}_{\mathrm{f}}\right) .
$$

Introducing (7.18) and integrating both sides of (7.17), we obtain

$$
\begin{aligned}
& \underline{x}\left(t_{f}\right)-\underline{x}(t)=c^{2}\left(M_{e}\left(t_{f}, t\right)-M_{p}\left(t_{f}, t\right)\right) \underline{x}\left(t_{f}\right) \Rightarrow \\
& {\left[I+c^{2}\left(M_{p}\left(t_{f}, t\right)-M_{e}\left(t_{f}, t\right)\right)\right] \underline{x}\left(t_{f}\right)=\underline{x}(t),}
\end{aligned}
$$

where

$$
M_{p}\left(t_{f}, t\right)=\int_{t}^{t_{f}} P\left(t_{f}, \tau\right) R_{p}^{-1} P^{\prime}\left(t_{f}, \tau\right) d \tau
$$

and

$$
M_{e}\left(t_{f}, t\right)=\int_{t}^{t_{f}} E\left(t_{f}, \tau\right) R_{e}^{-1} E^{\prime}\left(t_{f}, \tau\right) d \tau
$$

From (7.19) we can write

$$
\underline{x}\left(t_{f}\right)=F^{-1}\left(t_{f}, t\right) \underline{x}(t) \Rightarrow \underline{\lambda}(t)=c^{2} F^{-1}\left(t_{f}, t\right) \underline{x}(t)
$$

where the feedback gain matrix $F$ is of the form

$$
F\left(t_{f}, t\right)=\left[I+c^{2}\left(M_{p}\left(t_{f}, t\right)-M_{e}\left(t_{f}, t\right)\right)\right] .
$$


Here, we assume that the matrix $F$ is invertible. Substitution of (7.21) into (7.15a) and (7.15b) yields

$$
\begin{aligned}
& \underline{u}^{o}(t)=-K_{u}\left(t_{f}, t\right) \underline{x}(t) \\
& \underline{v}^{o}(t)=-K_{v}\left(t_{f}, t\right) \underline{x}(t)
\end{aligned}
$$

and

$$
J(t, \underline{x}(t))=\frac{c^{2}}{2} \underline{x}^{\prime}(t) F^{-1}\left(t_{f}, t\right) \underline{x}(t),
$$

where

$$
\begin{aligned}
& K_{u}\left(t_{f}, t\right)=c^{2} R_{p}^{-1} P^{\prime}\left(t_{f}, t\right) F^{-1}\left(t_{f}, t\right) \\
& K_{v}\left(t_{f}, t\right)=c^{2} R_{e}^{-1} E^{\prime}\left(t_{f}, t\right) F^{-1}\left(t_{f}, t\right)
\end{aligned}
$$

and $\underline{x}(t)$ is the predicted terminal miss at any given time $t$ which represents the position or the state of the game.

As seen, the optimal pursuit-evasion feedback controls (optimal strategies) are linear combinations of the predicted miss with the time-variant gain factors which measure the control capabilities of pursuer and evader on the predicted miss $\underline{x}(t)$. In addition, if a saddle point exists or the Equations (7.22) are to be considered as the strategies, then the non-singularities of $F\left(t_{f}, t\right)$ for all $t \in\left[t_{0}, t_{f}\right]$ is a sufficient condition for optimality and uniqueness of these strategies (movements of the game). The matrix $F$ is invertible if

$$
\operatorname{Det}\left(I+c^{2}\left(M_{p}\left(t_{f}, t\right)-M_{e}\left(t_{f}, t\right)\right)\right) \neq 0
$$

or 
$M_{p}\left(t_{f}, t\right)-M_{e}\left(t_{f}, t\right)$ is a positive definite matrix (see Equation (7.21b)). The latter says that some states of pursuer which are determined by matrix $C_{p}$ (states of interest) must be more positive definite (more controllable) than those of the evader which are determined by matrix $\mathrm{C}_{\mathrm{e}}$ (see Equations (7.16) \& (7.20)). The following example illustrates the general concept of a (deterministic) pursuitevasion problem.

\subsubsection{An Example Guidance Law} for Target Interception [80]

We assume that the equations of motion for an interceptor and target are described by Equations (7.24) and the payoff criterion is defined by Equation (7.25),

$$
\begin{aligned}
& \dot{r}_{p}(t)=v_{p}(t) ; \dot{v}_{p}(t)=f_{p}+a_{p}(t) \\
& \dot{r}_{e}(t)=v_{e}(t) ; \dot{v}_{e}(t)=f_{e}+a_{e}(t) \\
& J\left(a_{p}, a_{e}\right)=\frac{b}{2}\left[r_{p}\left(t_{f}\right)-r_{e}\left(t_{f}\right)\right]^{2}+\frac{1}{2} \int_{0}^{t_{f}}\left[\frac{1}{\gamma_{p}} a_{p}^{2}(t)-\frac{1}{\gamma_{e}} a_{e}^{2}(t)\right] d t
\end{aligned}
$$

where $\mathbf{r}$ and $\mathrm{v}$ are the position and the velocity of a body in the space, respectively, $f$ is the external force per unit per mass exerted on the body, $a$ is the acceleration of the body, and $\gamma_{p}$ and $\gamma_{e}$ are the energy capacity of the pursuer and evader, respectively. It is assumed that the altitude difference between the pursuer and the evader is small enough that $f_{p}=f_{e}$ and consequently, since only the difference $r_{p}(t)$ $r_{e}(t)$ is of interest in this problem, the effect of the external forces can 
be ignored.

We can develop the following state space models for the pursuer and the evader

$$
\begin{aligned}
& \underline{\dot{x}}_{p}(t)=A_{p} \underline{x}_{p}(t)+\underline{b}_{p} a_{p}(t) \\
& \underline{\dot{x}}_{e}(t)=A_{e} \underline{x}_{e}(t)+\underline{b}_{e} a_{e}(t)
\end{aligned}
$$

where

$$
\underline{x}_{p}=\left[\begin{array}{c}
r_{p} \\
v_{p}
\end{array}\right], \quad \underline{x}_{e}=\left[\begin{array}{c}
r_{e} \\
v_{e}
\end{array}\right], A_{p}=A_{e}=\left[\begin{array}{ll}
0 & 1 \\
0 & 0
\end{array}\right], \underline{b}_{p}=\underline{b}_{e}=\left[\begin{array}{l}
0 \\
1
\end{array}\right] .
$$

Equation (7.25) can be written

$$
\begin{aligned}
J\left(a_{p}, a_{e}\right)= & \frac{b}{2}\left[\left(\underline{x}_{p}\left(t_{f}\right)-\underline{x}_{e}\left(t_{f}\right)\right)^{\prime} A^{\prime} A\left(\underline{x}_{p}\left(t_{f}\right)-\underline{x}_{e}\left(t_{f}\right)\right)\right] \\
& +\frac{1}{2} \int_{0}^{t_{f}}\left[\frac{1}{c_{p}} a_{p}^{2}(t)-\frac{1}{c_{e}} a_{e}^{2}(t)\right] d t
\end{aligned}
$$

since, $r_{p}()=.A \underline{x}_{p}(),. r_{e}()=.A \underline{x}_{e}($.$) with C_{p}=C_{e}=A=\left[\begin{array}{ll}1 & 0\end{array}\right]$.

Now, in order to obtain the pair of saddle point $\left(a_{p}^{o}, a_{e}^{o}\right)$, we may apply the Equations (7.9), (7.10), (7.16), and (7.20)-(7.23) as follows.

$$
\begin{aligned}
& \Phi_{p}\left(t_{f}, t\right)=e^{A_{p}\left(t_{f}-t\right)}=I+A_{p}\left(t_{f}-t\right)=\left[\begin{array}{cc}
1 & t_{f}-t \\
0 & 1
\end{array}\right] \\
& \Phi_{e}\left(t_{f}, t\right)=e^{A_{c}\left(t_{f}-t\right)}=\left[\begin{array}{cc}
1 & t_{f}-t \\
0 & 1
\end{array}\right] \\
& P\left(t_{f}, t\right)=A \Phi_{p}\left(t_{f}, t\right) \underline{b}_{p}=\left[\begin{array}{ll}
1 & 0
\end{array}\right]\left[\begin{array}{cc}
1 & t_{f}-t \\
0 & 1
\end{array}\right]\left[\begin{array}{l}
0 \\
1
\end{array}\right]=t_{f}-t
\end{aligned}
$$




$$
\begin{aligned}
& E\left(t_{f}, t\right)=A \Phi_{e}\left(t_{f}, t\right) \underline{b}_{e}=\left[\begin{array}{ll}
1 & 0
\end{array}\right]\left[\begin{array}{ll}
1 & t_{f}-t \\
0 & 1
\end{array}\right]\left[\begin{array}{l}
0 \\
1
\end{array}\right]=t_{f}-t \\
& M_{p}\left(t_{f}, t\right)=\int_{t}^{t_{f}} P\left(t_{f}, \tau\right) \gamma_{p} P^{\prime}\left(t_{f}, \tau\right) d \tau= \\
& \quad \gamma_{p} \int_{t}^{t_{f}}\left(t_{f}-\tau\right)^{2} d \tau=\frac{\gamma_{p}}{3}\left(t_{f}-t\right)^{3} \\
& M_{e}\left(t_{f}, t\right)=\int_{t}^{t_{f}} E\left(t_{f}, \tau\right) \gamma_{e} E^{\prime}\left(t_{f}, \tau\right) d \tau= \\
& F\left(t_{f}, t\right)=\left[I+b\left(M_{p}\left(t_{f}, t\right)-M_{e}\left(t_{f}, t\right)\right)\right]=1+b \frac{\gamma_{p}-\gamma_{e}}{3}\left(t_{f}-t\right)^{3} \\
& K_{a_{p}}\left(t_{f}, t\right)=b \gamma_{p} P^{\prime}\left(t_{f}, t\right) F^{-1}\left(t_{f}, t\right)=\frac{b \gamma_{p}\left(t_{f}-t\right)}{1+\left(\gamma_{p}-\gamma_{e}\right)\left(t_{f}-t\right)^{3} b / 3} \\
& K_{a_{e}}\left(t_{f}, t\right)=b \gamma_{e} E^{\prime}\left(t_{f}, t\right) F^{-1}\left(t_{f}, t\right)=\frac{b \gamma_{e}\left(t_{f}-t\right)}{1+\left(\gamma_{p}-\gamma_{e}\right)\left(t_{f}-t\right)^{3} b / 3} \\
& \underline{x}(t)=A\left[\Phi_{p}\left(t_{f}, t\right) \underline{x}_{p}(t)-\Phi_{e}\left(t_{f}, t\right) \underline{x}_{e}(t)\right] \\
& =\left[\begin{array}{ll}
1 & 0]
\end{array}\right]\left[\begin{array}{ll}
1 & t_{f}-t \\
0 & 1
\end{array}\right]\left[\begin{array}{l}
r_{p}(t)-r_{e}(t) \\
v_{p}(t)-v_{e}(t)
\end{array}\right] \\
& =r_{p}(t)-r_{e}(t)+\left(t_{f}-t\right)\left[v_{p}(t)-v_{e}(t)\right] .
\end{aligned}
$$

Thus, the pair of saddle points becomes

$$
\begin{aligned}
a_{p}^{0}(t) & =-K_{a_{p}}\left(t_{f}, t\right) \underline{x}(t) \\
& =-\frac{b \gamma_{p}\left(t_{f}-t\right)\left\{r_{p}(t)-r_{e}(t)+\left(t_{f}-t\right)\left[v_{p}(t)-v_{e}(t)\right]\right\}}{1+\left(\gamma_{p}-\gamma_{e}\right)\left(t_{f}-t\right)^{3} b / 3}, \\
a_{e}^{0}(t) & =-K_{a_{e}}\left(t_{f}, t\right) \underline{x}(t) \\
& =-\frac{b \gamma_{e}\left(t_{f}-t\right)\left\{r_{p}(t)-r_{e}(t)+\left(t_{f}-t\right)\left[v_{p}(t)-v_{e}(t)\right]\right\}}{1+\left(\gamma_{p}-\gamma_{e}\right)\left(t_{f}-t\right)^{3} b / 3}
\end{aligned}
$$




$$
=\frac{\gamma_{\mathrm{e}}}{\gamma_{\mathrm{p}}} \mathrm{a}_{\mathrm{p}}^{0}(\mathrm{t})
$$

From Equations (7.28) it can be seen that the existence and uniqueness of the optimal strategies are assured if

$$
1+\left(\gamma_{p}-\gamma_{e}\right)\left(t_{f}-t\right)^{3} b / 3 \neq 0, \forall t \in\left[0, t_{f}\right]
$$

Let us determine under what condition the above equation becomes zero,

$$
\begin{aligned}
& 1+\left(\gamma_{p}-\gamma_{e}\right)\left(t_{f}-t\right)^{3} b / 3=0, \forall t \in\left[0, t_{f}\right] \Rightarrow \\
& t=t_{f}+\sqrt[3]{\frac{3}{b\left(\gamma_{p}-\gamma_{e}\right)}} .
\end{aligned}
$$

If $\gamma_{p}>\gamma_{e}$, then $t>t_{f}$ and for $\gamma_{p}<\gamma_{e}, t$ becomes less than $t_{f}$. Therefore, for the case in which the pursuer has more energy than the evader, $\gamma_{p}>\gamma_{e}$, the feedback gain matrix $F$ is always positive in the range between 0 to $t_{f}$ and the interception is guaranteed, especially for large b. But for the second case, $\gamma_{\mathrm{p}}<\gamma_{\mathrm{e}}$, there is no guarantee for the interception, especially for large $t_{f}$ the interception is impossible.

Figure 7.1 illustrates a computer simulation of the target interception problem discussed above. The top path, represents the case in which $\gamma_{p}<\gamma_{e}$. We can see the impossibility of interception. The bottom paths of Figure 7.1 represent the cases in which $\gamma_{p}>\gamma_{e}$ As seen the interception is guaranteed by increasing $\gamma_{p}$ which indicates the attempts of the pursuer by spending more energy in order to capture the evader. The experimental results totally meet the theoretical results which we discussed in this section. 

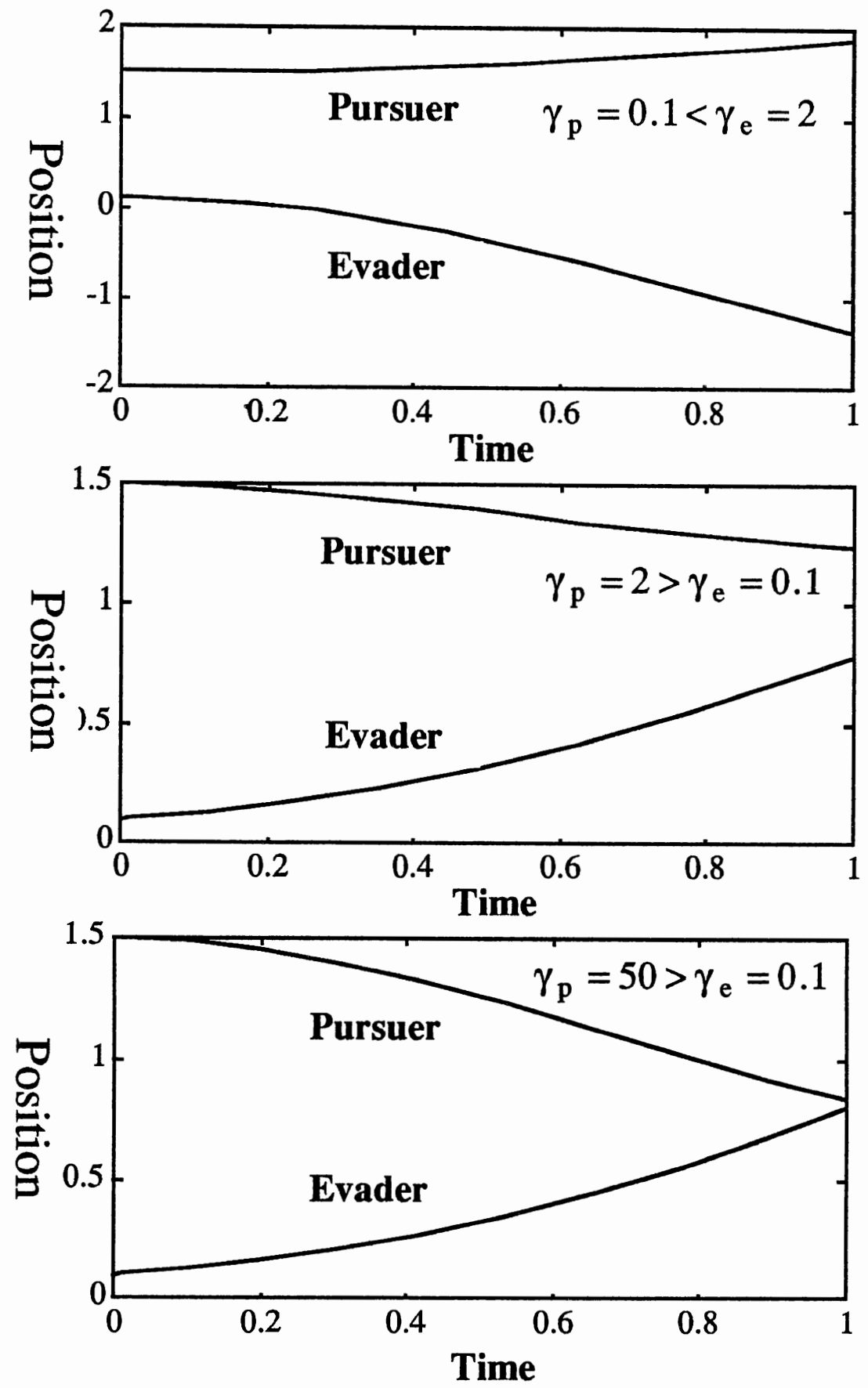

Figure 7.1 Simulation of a Simple Pursuit-Evasion (Target Interception) Problem, $b=1 ; t_{f}=1 \mathrm{sec}$. 


\subsection{Linear Stochastic Differential Games}

\subsubsection{Introduction}

The previous section considered a linear deterministic twoplayer, zero-sum pursuit-evasion differential game in which each player is assumed to have perfect knowledge of his and his opponent's states (dynamics). This section considers a linear stochastic quadratic pursuit-evasion game in which the system may be disturbed by additive noise and both the evader and pursuer have access to noisecorrupted measurements.

Reference [81] initiated the concept of stochastic differential game by presenting some results under the general subject of differential games with incomplete information. Ref. [99], by investigating a minimax index of performance for designing a filter to estimate a noisy signal with bounded accelerations, actually solved a simple stochastic differential game. A more general stochastic linear differential game in which only one player controlled the state was formulated by authors of references [97] and [98] by applying calculus of variation optimization techniques. References [101-102] present the most important results for stochastic differential games in which one player has access to measurements corrupted by white noise and the other one has perfect measurements. For the case in which both players have noisy-measurements, references [104] and [105] present a solution. The next section reviews the stochastic differential games considered by references [101] and [105] because of their important results. 


\subsubsection{Problem Statement}

In this section, we review the main results of References [101] \& [105]. Consider the linear systems given in (7-5) \& (7-6) which define, respectively the dynamics of pursuer and evader. It is assumed that the pursuer knows the dynamics and states of both himself and his opponent, while the evader has imperfect knowledge of his and his opponent's state and dynamics. The measurements available to the evader are assumed to be of the form

$$
\underline{z}(t)=H \underline{x}(t)+\underline{v}_{x}(t)
$$

where $\underline{x}(t)$ is the terminal miss whose dynamic is given in (7.11) which defines the pursuit-evasion system, and $\underline{v}_{x}(t)$ is a Gaussian white noise with zero mean and covariance matrix $V_{x}$. From (7.9) and (7.29), we may write

$$
\begin{aligned}
& \underline{z}_{p}(t)=H_{p} \underline{x}_{p}(t)+\underline{v}_{p}(t) \\
& \underline{z}_{e}(t)=H_{e} \underline{x}_{e}(t)+\underline{v}_{e}(t),
\end{aligned}
$$

which reflect the imperfect measurements of the evader on his and the pursuer's state, where $\underline{v}_{p}$ and $\underline{v}_{e}$ are white, zero mean Gaussian noise vectors. Therefore, the class of evader's admissible strategies at time $t$ is based on the measurement $\underline{z}(\tau), \tau \in\left[t_{0}, t\right]$. Based on these measurements, let the optimal mean squared linear estimate of $\underline{x}(t)$ and the estimation error be denoted as : 


$$
\begin{aligned}
& \underline{\hat{x}}(t)=E\left\{\underline{x}(t) \text { given } \underline{z}(\tau), t_{0} \leq \tau \leq t\right\} \\
& \underline{\tilde{x}}(t)=\underline{x}(t)-\underline{\hat{x}}(t) .
\end{aligned}
$$

The stochastic pursuit-evasion problem is defined as: for the linear system defined in (7.11) with the evader having noisy measurement given in (7.29) and the pursuer having perfect knowledge of $\underline{x}(t)$, find a pair of saddle points $\left(\underline{u}^{0}, \underline{v}^{0}\right)$ such that the following quadratic criterion is minimized

$$
J=E\left\{\begin{aligned}
\frac{c^{2}}{2} \underline{x}^{\prime}\left(t_{f}\right) \underline{x}\left(t_{f}\right) & \\
& +\frac{1}{2} \int_{t_{0}}^{t_{f}} \underline{u}^{\prime}(t) R_{p} \underline{u}(t) d t-\frac{1}{2} \int_{t_{0}}^{t_{f}} \underline{v}^{\prime}(t) R_{e} \underline{v}(t) d t
\end{aligned}\right\},
$$

where $\underline{x}\left(t_{f}\right)$ is a random variable, because the initial state $\underline{x}\left(t_{0}\right)$ for the evader is assumed to be a Gaussian random vector, uncorrelated with $\underline{v}(t)$ for all $t \in\left[t_{0}, t_{f}\right]$, with a mean $\underline{x}_{0}$ and a covariance $P_{0}$.

It is assumed that the optimal strategies are of the structural form

$$
\begin{aligned}
& \underline{\underline{u}}^{o}(t)=K_{p}\left(t, t_{f}\right) \underline{x}(t)+L_{p}\left(t, t_{f}\right) \underline{\tilde{x}}(t) \\
& \underline{v}^{o}(t)=K_{e}\left(t, t_{f}\right) \underline{\hat{x}}(t) .
\end{aligned}
$$

Equation (7.31a) says that we implicitly assume that to the pursuer, the error of the evader's estimate is available at time $t$. In order to see under what conditions the pursuer is able to obtain the exact value of 
the error, we may consider the following equation which is obtained by substitution (7.31) into (7.11)

$$
E\left(t_{f}, t\right) K_{e}\left(t_{f}, t\right) \underline{\hat{x}}(t)=P\left(t_{f}, t\right) \underline{u}(t)-\underline{\dot{x}}(t)
$$

where $E$ and $P$ are given in (7.16).

Figure 7.2 illustrates the realization of the above control strategies for the stochastic pursuit-evasion problem. By looking at Figure 7.2 , we can write the following equation which produces $\underline{u}(t)$,

$$
\underline{u}(t)=\left(I-K_{e}^{-1} E^{-1} P\right)^{-1}\left[\left(K_{p}+L_{p}\right) \underline{x}+L_{p} K_{e}^{-1} E^{-1} \underline{\dot{x}}\right] .
$$

From equations (7.32) we can see that the pursuer can obtain $\underline{\hat{x}}(t)$ if the gain matrices $E$ and $K_{e}$ and the matrix $\left(I-K_{e}^{-1} E^{-1} P\right)$ are nonsingular. We shall discuss these conditions after the determination of $\mathbf{K}_{\mathbf{e}}$.

\subsubsection{The Solution}

This section presents the solution of the stochastic differential game stated in the previous section. Introducing Equations (7.16), (7.30b) and using (7.31), the pursuit-evasion system equation (7.11) and the index (7.30) become

$$
\underline{\dot{x}}(t)=\left[P K_{p}-E K_{e}\right] \underline{x}(t)+\left[P L_{p}+E K_{e}\right] \underline{\tilde{x}}(t),
$$




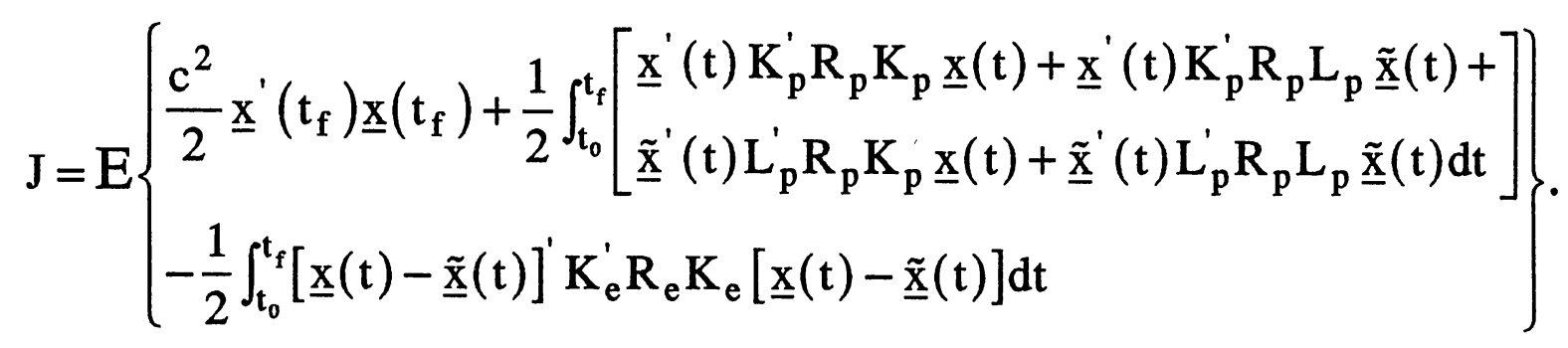

By letting $X(t)=E\left\{\underline{x}(t) \underline{x}^{\prime}(t)\right\}$ and knowing $X^{\prime} A X=\operatorname{Tr}\left\{X X^{\prime} A\right\}$, the index $J$ in (7.34) and $\dot{X}(t)$ can be rewritten as:

$$
\begin{gathered}
J=\operatorname{Tr}\left\{\begin{array}{l}
\frac{c^{2}}{2} X\left(t_{f}\right)+\frac{1}{2} \int_{t_{0}}^{t_{f}}\left[X(t)\left(K_{p}^{\prime} R_{p} K_{p}-K_{e}^{\prime} R_{e} K_{e}\right) d t\right] \\
+\frac{1}{2} \int_{t_{0}}^{t_{f}}\left[Q(t)\left(K_{p}^{\prime} R_{p} L_{p}+L_{p}^{\prime} R_{p} K_{p}+L_{p}^{\prime} R_{p} L_{p}+K_{e}^{\prime} R_{e} K_{e}\right)\right] d t
\end{array}\right\} \\
\dot{X}(t)= \\
P K_{p} X+X K_{p}^{\prime} P^{\prime}+Q L_{p}^{\prime} P^{\prime}+P L_{p} Q \\
+E K_{e}(Q-X)+(Q-X) K_{e}^{\prime} E^{\prime}
\end{gathered}
$$

where $Q(t)$ is the covariance matrix of the evader's estimate error

$$
\mathrm{Q}(\mathrm{t})=\mathrm{E}\left\{\underline{\tilde{x}}(\mathrm{t}) \underline{\tilde{x}}^{\prime}(\mathrm{t})\right\}=\mathrm{E}\left\{\underline{\mathrm{x}}(\mathrm{t}) \underline{\tilde{x}}^{\prime}(\mathrm{t})\right\}, \sin \operatorname{ce} \mathrm{E}\left\{\underline{\hat{\mathrm{x}}}(\mathrm{t}) \underline{\tilde{x}}^{\prime}(\mathrm{t})\right\}=0,
$$

and it satisfies the following Riccati equation

$$
\dot{Q}(t)=P\left(K_{p}+L_{p}\right) Q+Q\left(K_{p}^{\prime}+L_{p}^{\prime}\right) P^{\prime}-Q H^{\prime} V_{x}^{-1} H Q
$$

Based on the system given in (7.33) \& (7.29), the following Kalman filter defines the evader's estimate

$$
\underline{\dot{\hat{x}}}(t)=\left[P K_{p}-E K_{e}\right] \underline{\hat{x}}(t)+Q H^{\prime} V_{x}^{-1}[\underline{z}(t)-H \underline{\hat{x}}(t)]
$$




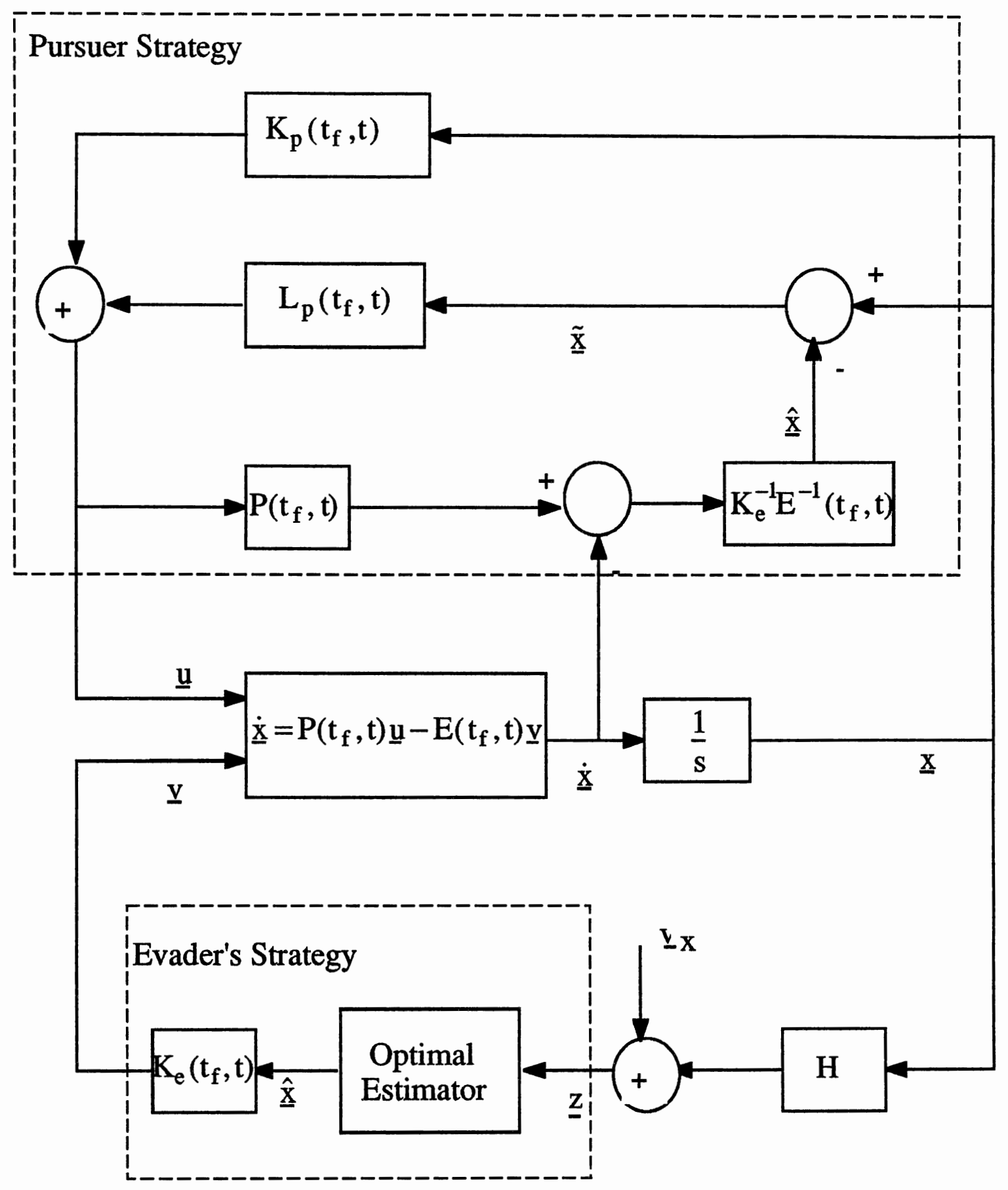

Figure 7.2. Stochastic Pursuit-Evasion Structure 
The original stochastic pursuit-evasion problem is now equivalent to the deterministic problem which is stated as: For the linear systems given in (7.36) \& (7.38), find a saddle point which minimaximizes the index given in (7.35).

As discussed in the previous sections, this problem may be solved by defining the Hamiltonian

$$
H=\frac{1}{2} \operatorname{Tr}\left\{\begin{array}{l}
X\left(K_{p}^{\prime} R_{p} K_{p}-K_{e}^{\prime} R_{e} K_{e}\right)+\Lambda_{1} \dot{X}+\Lambda_{2} \dot{Q} \\
Q\left(K_{p}^{\prime} R_{p} L_{p}+L_{p}^{\prime} R_{p} K_{p}+L_{p}^{\prime} R_{p} L_{p}+K_{e}^{\prime} R_{e} K_{e}\right)
\end{array}\right\} .
$$

By defining $S_{1}=\Lambda_{1}+\Lambda_{1}^{\prime}$ and $S_{2}=\Lambda_{2}+\Lambda_{2}^{\prime}$, it can be shown that the following matrix differential equations solve for optimal strategies as:

$$
\begin{aligned}
\dot{S}_{1}= & S_{1}\left(P R_{p}^{-1} P-E R_{e}^{-1} E^{\prime}\right) S_{1} \\
\dot{S}_{2}= & S_{2} P_{p}^{-1} P_{2}+S_{2}\left(P_{p}^{-1} P_{1}+Q^{\prime} Q^{-1} H\right)+ \\
& \left(S_{1} P R_{p}^{-1} P+H^{\prime} Q^{-1} H Q\right) S_{2}+S_{1} E R_{e}^{-1} E^{\prime} S_{1} \\
\dot{Q}(t) & =P\left(K_{p}+L_{p}\right) Q+Q\left(K_{p}^{\prime}+L_{p}^{\prime}\right) P^{\prime}-Q^{\prime} V_{x}^{-1} H Q \\
K_{p}= & -R_{p}^{-1} P_{1} \\
K_{e}= & -R_{e}^{-1} E_{1} \\
L_{p}= & -R_{p}^{-1} P_{2}
\end{aligned}
$$

with the boundary conditions

$$
S_{1}\left(t_{f}\right)=\frac{c^{2}}{2} I, S_{2}\left(t_{f}\right)=0 \text { and } Q\left(t_{0}\right)=Q_{0}
$$


Therefore, the optimal strategies are obtained as:

$$
\begin{aligned}
\underline{u}^{o}(t) & =K_{p}\left(t_{f}, t\right) \underline{x}(t)+L_{p}\left(t_{f}, t\right) \underline{\tilde{x}}(t) \\
& =-R_{p}^{-1} P^{\prime}\left(t_{f}, t\right) S_{1}(t) \underline{x}(t)-R_{e}^{-1} E^{\prime}\left(t_{f}, t\right) S_{2} \underline{\tilde{x}}(t), \\
\underline{v}^{o}(t) & =-R_{e}^{-1} E^{\prime}\left(t_{f}, t\right) S_{1}(t) \underline{\hat{x}}(t),
\end{aligned}
$$

where $E($.$) and P($.$) are given in (7.16). Finally, by substituting the$ optimal values $K_{p}, L_{p}$, and $K_{e}$ given in (7.42) into the payoff index (7.35) and after some algebraic manipulations and simplifications, we can find the optimal expected cost expression as:

$$
\begin{aligned}
& J\left(\underline{\mathbf{u}}^{0}, \underline{v}^{0}\right)=\frac{1}{2}\left\{\begin{array}{l}
\underline{x}^{\prime}\left(t_{0}\right) S_{1}\left(t_{0}\right) \underline{x}\left(t_{0}\right)+\underline{\tilde{x}}^{\prime}\left(t_{0}\right) S_{2}\left(t_{0}\right) \underline{\tilde{x}}\left(t_{0}\right)+ \\
\operatorname{Tr}\left\{\int_{t_{0}}^{t_{f}} Q(t) H^{\prime} V_{x}^{-1} H Q(t) S_{2}(t) d t\right\}
\end{array}\right\} \Rightarrow \\
& J\left(\underline{\underline{u}}^{o}, \underline{v}^{0}\right)=\frac{1}{2} \operatorname{Tr}\left\{\begin{array}{l}
S_{1}\left(t_{0}\right) X\left(t_{0}\right)+S_{2}\left(t_{0}\right) Q\left(t_{0}\right)+ \\
\int_{t_{0}}^{t_{f}} Q(t) H^{\prime} V_{x}^{-1} H Q(t) S_{2}(t) d t
\end{array}\right\} .
\end{aligned}
$$

By introducing (7.43) and comparing (7.22c) and (7.45), we can see that the first term in (7.45) corresponds to the total value for the index in the deterministic case ( since it can be shown that $S_{1}=F^{-1}$ ), and the second and third terms represent the total uncertainty of the evader's estimate initially and during the play of the game. It can be shown that these two terms are negative, since $S_{2}$ is a negative definite matrix. Therefore, the last two terms in (7.45), which is called the relative or reduced payoff, represent the reduction in the capability of the evader. 
From Equations (7.42a) and (7.32), we can conclude that the pursuer can be given $\underline{\hat{x}}$ if 1 ) the interested states and the evader's control input, $\underline{v}$, are of the same dimension, and 2) the matrices $E$ and $S_{1}$ are invertible.

Equations (7.41) through (7.42) with boundary conditions given in (7.43) present the solution for the stochastic differential game defined by Equations (7.11) and (7.29-31). This solution is called the prior commitment type [106] and it is based on the assumptions that 1) the pursuer is given, or can determine exactly, the error in the evader's estimate (i.e., the pursuer takes the advantage of this error), and 2) the evader's control is of a feedback form based on the estimate of the pursuit-evasion system's state, $\underline{x}(t)$. This is not an online solution because of the uncoupled nonlinear differential equations (7.41) with two different boundary conditions given in (7.43). These nonlinear differential equations can be solved by methods presented in the References [110-112]. The following section presents an on-line solution to the same differential game and the games where both players have access to noisy measurements.

\subsubsection{Delayed Commitment Solution}

Consider the pursuit-evasion system whose dynamics is defined b y

$$
\begin{aligned}
& \underline{\dot{x}}(t)=P(t) \underline{u}(t)-E(t) \underline{v}(t), \\
& \underline{z}_{p}(t)=H_{p} \underline{x}(t)+\underline{v}_{p}(t), \\
& \underline{z}_{e}(t)=H_{e} \underline{x}(t)+\underline{v}_{e}(t),
\end{aligned}
$$


where the zero mean, white noises $\underline{v}_{p}(t)$ and $\underline{v}_{e}(t)$ are Gaussian and independent, with covariance matrices $V_{p}$ and $V_{e}$, respectively. It is assumed that the initial state $\underline{x}\left(t_{0}\right)$ is a Gaussian white noise, uncorrelated with both measurement noises, and it has a mean of $\underline{x}_{0}$ and a covariance matrix $\mathrm{P}_{\mathrm{x} 0}$. The pursuer, the minimizer player controlling $u$, has available measurements given in (7.46b) and the available measurements for the evader, the maximizer player controlling $\mathrm{v}$, is given in $(7.46 \mathrm{c})$. The payoff criterion to the game is assumed to be of the quadratic form given in (7.31).

By following the procedure given in [105], the pursuer's optimal strategy is obtained by solving of the following set of nonlinear equations:

$$
\begin{aligned}
\underline{u}^{*}(t)= & -R_{p}^{-1} P^{\prime}(t) S(t) \underline{\hat{x}}_{p}(t), \\
\dot{S}(t)= & S(t)\left[P(t) R_{p}^{-1} P^{\prime}(t)-E(t) R_{e}^{-1} E^{\prime}(t)\right] S(t), \quad S\left(t_{f}\right)=c^{2} I \\
\dot{N}_{p}(t)= & N_{p}(t) E(t) R_{e}^{-1} E^{\prime}(t) N_{p}(t), N_{p}\left(t_{f}\right)=I \\
\dot{\Gamma}_{p}(t)= & -N_{p}(t) E(t) R_{e}^{-1} E^{\prime}(t) \Gamma_{p}(t)-N_{p}(t) P(t) R_{p}^{-1} P^{\prime}(t) S(t)+ \\
& \Gamma_{p}(t)\left[P(t) R_{p}^{-1} P^{\prime}(t)-E(t) R_{e}^{-1} E^{\prime}(t)\right] S(t), \quad \Gamma_{p}\left(t_{f}\right)=0 \\
\dot{\hat{x}}_{p}(t)= & {\left[\begin{array}{ll}
P(t) R_{p}^{-1} P^{\prime}(t) S(t)+E(t) R_{e}^{-1} E^{\prime}(t) N_{p}(t) \\
-E(t) R_{e}^{-1} E^{\prime}(t) \Gamma_{p}(t)
\end{array}\right.} \\
& Q_{p 1}(t) H_{p}^{\prime} V_{p}^{-1}\left[\underline{x}_{p}(t)-H_{p} \hat{x}_{p}(t)\right], \quad \hat{x}_{p}\left(t_{0}\right)=\underline{x}_{0}, \\
\dot{Q}_{p 1}(t)= & E(t) R_{e}^{-1} E^{\prime}(t) N_{p}(t) Q_{p 2}(t)+Q_{p 2}(t) N_{p}(t) E(t) R_{e}^{-1} E^{\prime}(t)- \\
& \quad Q_{p 1}(t) H_{p}^{\prime} V_{p}^{-1} H_{p} Q_{p 1}(t), \quad Q_{p 1}\left(t_{0}\right)=P_{x 0},
\end{aligned}
$$


$(7.47 f)$

$$
\begin{gathered}
\dot{Q}_{p 2}(t)=E(t) R_{e}^{-1} E^{\prime}(t) N_{p}(t) Q_{p 2}(t)+Q_{p 2}(t) N_{p}(t) E(t) R_{e}^{-1} E^{\prime}(t)- \\
Q_{p 1}(t) H_{p}^{\prime} V_{p}^{-1} H_{p} Q_{p 2}(t)-Q_{p 2}(t) H_{p}^{\prime} V_{p}^{-1} H_{p} Q_{p 1}(t)+ \\
Q_{p 2}(t) H_{p}^{\prime} V_{p}^{-1} H_{p} Q_{p 2}(t)+Q_{p 1}(t) H_{e}^{\prime} V_{e}^{-1} H_{e} Q_{p 1}(t)- \\
Q_{p 1}(t) H_{e}^{\prime} V_{e}^{-1} H_{e} Q_{p 2}(t)-Q_{p 2}(t) H_{e}^{\prime} V_{e}^{-1} H_{e} Q_{p 1}(t)+ \\
+Q_{p 2}(t) H_{e}^{\prime} V_{e}^{-1} H_{e} Q_{p 2}(t), \quad Q_{p 2}\left(t_{f}\right)=0
\end{gathered}
$$

The corresponding equations which solve for the optimal strategy of the evader are:

$$
\begin{aligned}
\underline{v}^{*}(t)= & -R_{e}^{-1} E^{\prime}(t) S(t) \hat{x}_{e}(t), \\
\dot{S}(t)= & S(t)\left[E(t) R_{e}^{-1} E^{\prime}(t)-P(t) R_{p}^{-1} P^{\prime}(t)\right] S(t), \quad S\left(t_{f}\right)=c^{2} I \\
\dot{N}_{e}(t)= & N_{e}(t) P(t) R_{p}^{-1} P^{\prime}(t) N_{e}(t), \quad N_{e}\left(t_{f}\right)=I, \\
\dot{\Gamma}_{e}(t)= & -N_{e}(t) P(t) R_{p}^{-1} P^{\prime}(t) \Gamma_{e}(t)-N_{e}(t) E(t) R_{e}^{-1} E^{\prime}(t) S(t)+ \\
& \Gamma_{e}(t)\left[E(t) R_{e}^{-1} E^{\prime}(t)-P(t) R_{p}^{-1} P^{\prime}(t)\right] S(t), \quad \Gamma_{e}\left(t_{f}\right)=0 \\
\dot{\hat{x}}_{e}(t)= & {\left[\begin{array}{ll}
\left.E(t) R_{e}^{-1} E^{\prime}(t) S(t)+P(t) R_{p}^{-1} P^{\prime}(t) N_{e}(t)\right] \\
-P(t) R_{p}^{-1} P^{\prime}(t) \Gamma_{e}(t)
\end{array}\right.} \\
& Q_{e 1}(t) H_{e}^{\prime} V_{e}^{-1}\left[\underline{z}_{e}(t)-H_{e} \hat{x}_{e}(t)\right], \quad \underline{\hat{x}}_{e}\left(t_{0}\right)=\underline{x}_{0}, \\
\dot{Q}_{e 1}(t)= & P(t) R_{p}^{-1} P^{\prime}(t) N_{e}(t) Q_{e 2}(t)+Q_{e 2}(t) N_{e}(t) P(t) R_{p}^{-1} P^{\prime}(t)- \\
& Q_{e 1}(t) H_{e}^{\prime} V_{e}^{-1} H_{e} Q_{e 1}(t), \quad Q_{e 1}\left(t_{0}\right)=P_{x 0},
\end{aligned}
$$




$$
\begin{aligned}
\dot{Q}_{e 2}(t)= & P(t) R_{p}^{-1} P^{\prime}(t) N_{e}(t) Q_{e 2}(t)+Q_{e 2}(t) N_{e}(t) P(t) R_{p}^{-1} P^{\prime}(t)- \\
& Q_{e 1}(t) H_{e}^{\prime} V_{e}^{-1} H_{e} Q_{e 2}(t)-Q_{e 2}(t) H_{e}^{\prime} V_{e}^{-1} H_{e} Q_{e 1}(t)+ \\
& Q_{e 2}(t) H_{e}^{\prime} V_{e}^{-1} H_{e} Q_{e 2}(t)+Q_{e 1}(t) H_{p}^{\prime} V_{p}^{-1} H_{p} Q_{e 1}(t)- \\
& Q_{e 1}(t) H_{p}^{\prime} V_{p}^{-1} H_{p} Q_{e 2}(t)-Q_{e 2}(t) H_{p}^{\prime} V_{p}^{-1} H_{p} Q_{e 1}(t)+ \\
& +Q_{e 2}(t) H_{p}^{\prime} V_{p}^{-1} H_{p} Q_{e 2}(t), \quad Q_{e 2}\left(t_{f}\right)=0
\end{aligned}
$$

The Equations (7.47) \& (7.48) represent an on-line solution of the differential game defined in Equations (7.46), because the above nonlinear matrix differential equations can be solved by applying either forward integration or backward integration.

In the case where the pursuer has perfect information of the state vector $x(t)$ and the evader has measurements given in $(7.47 \mathrm{c})$, it can be shown that the following equations solve for the optimal strategies for both players

$$
\begin{aligned}
& \underline{\mathrm{u}}^{*}(t)=-R_{\mathrm{p}}^{-1} P^{\prime}(t) S(t) \underline{\hat{x}}(t)+R_{p}^{-1} P^{\prime}(t) N(t) \underline{\tilde{x}}(t), \\
& \underline{v}^{*}(t)=-R_{e}^{-1} E^{\prime}(t) S(t) \underline{\hat{x}}(t), \\
& \dot{S}(t)= S(t)\left[P(t) R_{p}^{-1} P^{\prime}(t)-E(t) R_{e}^{-1} E^{\prime}(t)\right] S(t), \quad S\left(t_{f}\right)=c^{2} I, \\
& \dot{N}(t)= N(t) P(t) R_{p}^{-1} P^{\prime}(t) N(t), \quad N\left(t_{f}\right)=I, \\
& \dot{Q}(t)=-P(t) R_{p}^{-1} P^{\prime}(t) N(t) Q(t)-Q(t) N(t) P(t) R_{p}^{-1} P^{\prime}(t)- \\
& \quad Q(t) H_{e}^{\prime} V_{e}^{-1} H_{e} Q(t), \quad Q\left(t_{0}\right)=P_{x 0}, \\
& \underline{\hat{x}}(t)=\left[E(t) R_{e}^{-1} E^{\prime}(t)-P(t) R_{p}^{-1} P^{\prime}(t)\right] S(t) \underline{\hat{x}}(t) \\
&+Q(t) H_{e}^{\prime} V_{e}^{-1}\left[\underline{z}_{e}(t)-H_{e} \underline{\hat{x}}_{e}(t)\right], \quad \underline{\hat{x}}\left(t_{0}\right)=\underline{x}_{0} .
\end{aligned}
$$


The optimal payoff for the evader is:

$$
\begin{aligned}
\mathrm{J}\left(\underline{\underline{u}}^{*}(\mathrm{t}), \underline{\mathrm{v}}^{*}(\mathrm{t})\right)= & \frac{1}{2} \underline{x}^{\prime}(\mathrm{t}) \mathrm{S}(\mathrm{t}) \underline{\mathrm{x}}(\mathrm{t})+\frac{1}{2} \operatorname{Tr}(\mathrm{N}(\mathrm{t}) \mathrm{Q}(\mathrm{t})) \\
& +\frac{1}{2} \operatorname{Tr}\left(\int_{\mathrm{t}}^{\mathrm{t}_{\mathrm{f}}}[\mathrm{N}(\tau)-\mathrm{S}(\tau)] \mathrm{Q}(\tau) \mathrm{H}_{\mathrm{e}}^{\prime} \mathrm{V}_{\mathrm{e}}^{-1} \mathrm{H}_{\mathrm{e}} \mathrm{Q}(\tau) \mathrm{d} \tau\right)
\end{aligned}
$$

\subsection{An Example of a Stochastic Differential Game}

In this section we apply the two types of solutions reviewed in the previous sections to an example of a stochastic pursuit-evasion game taken from [101] and compare the prior-commitment and the delayed-commitment results. This is a game in two-dimensional Euclidian space, where the pursuer, the minimizer player, has perfect information of his and his opponent's system, while the evader, the maximizer player, is given only noise-corrupted measurements.

The dynamics of the pursuer and the evader are given by

$$
\begin{array}{lll}
\underline{\dot{x}}_{p}(t)=A_{p} \underline{x}_{p}(t)+B_{p} \underline{u}(t) & , & \underline{x}_{p}\left(t_{0}\right)=\underline{x}_{p_{0}} \\
\underline{\dot{x}}_{e}(t)=A_{e} \underline{x}_{e}(t)+B_{e} \underline{v}(t) \quad, & \underline{x}_{e}\left(t_{0}\right)=\underline{x}_{e} 0
\end{array}
$$

where

$$
A_{p}=A_{e}=\left[\begin{array}{llll}
0 & 0 & 1 & 0 \\
0 & 0 & 0 & 1 \\
0 & 0 & 0 & 0 \\
0 & 0 & 0 & 0
\end{array}\right], B_{p}=B_{e}=\left[\begin{array}{ll}
0 & 0 \\
0 & 0 \\
1 & 0 \\
0 & 1
\end{array}\right],
$$

and the sate vectors of the pursuer and the evader are 


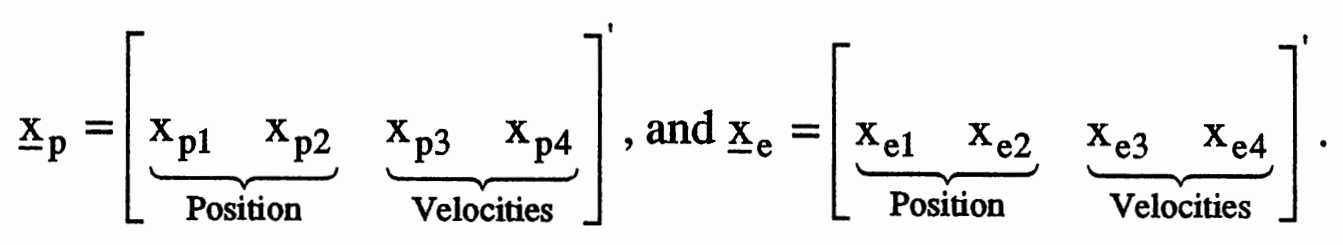

For this game the terminal position miss is of interest. Therefore, the matrices $C_{p}$ and $C_{e}$ which give the reduced (interested) state vectors are

$$
C_{p}=C_{e}=\left[\begin{array}{llll}
1 & 0 & 0 & 0 \\
0 & 1 & 0 & 0
\end{array}\right],
$$

and the payoff index for this game can be written as:

$$
J\left(\underline{u}, \underline{v} ; t_{0}, \underline{x}_{0}\right)=E\left\{\begin{array}{l}
\frac{c^{2}}{2}\left[\left(x_{p 1}-x_{e 1}\right)^{2}+\left(x_{p 2}-x_{e 2}\right)^{2}\right]+ \\
\frac{1}{2} \int_{t_{0}}^{t_{f}} \underline{u}^{\prime}(t) R_{p} \underline{u}(t) d t-\frac{1}{2} \int_{t_{0}}^{t_{f}} \underline{v}^{\prime}(t) R_{e} \underline{v}(t) d t
\end{array}\right\},
$$

where, it is assumed that the energy weighting matrices are of the form

$$
R_{p}=\left[\begin{array}{cc}
r_{1 p} & 0 \\
0 & r_{2 p}
\end{array}\right] \text { and } R_{e}=\left[\begin{array}{cc}
r_{1 e} & 0 \\
0 & r_{2 e}
\end{array}\right]
$$

Using (7.10) \& (7.16), it can be shown that

$$
P\left(t_{f}, t\right)=E\left(t_{f}, t\right)=\left[\begin{array}{cc}
t_{f}-t & 0 \\
0 & t_{f}-t
\end{array}\right],
$$

and the Equation (7.9), reduced pursuit-evasion system, becomes 


$$
\underline{x}(t)=\left[\begin{array}{cccc}
1 & 0 & t_{f}-t & 0 \\
0 & 1 & 0 & t_{f}-t
\end{array}\right]\left[\underline{x}_{p}(t)-\underline{x}_{e}(t)\right]
$$

Since $E($.$) in invertible for all t$ less that $t_{f}$ and since $\underline{x}(t)$ and control vectors are of the same dimension 2 , we can apply the priorcommitment solution to the problem given in equations (7.52) and (7.29) with criterion $\mathrm{J}$ given in (7.51).

In this example the constants and the parameters are:

$$
\begin{array}{ll}
t_{0}=\text { Initial time } & =0 \mathrm{sec} . \\
t_{\mathrm{f}}=\text { Final time } & =10 \mathrm{sec} . \\
\mathrm{c}^{2}=\text { Terminal miss weighting factor } & =10 \mathrm{ft}^{-2} \\
\mathrm{r}_{1 \mathrm{p}}=\text { Pursuer control weighting factor } & =0.4 \mathrm{ft}^{-2} \mathrm{sec}^{3} \\
\mathrm{r}_{1 \mathrm{e}}=\text { Evader control weighting factor } & =1 \mathrm{ft}^{-2} \mathrm{sec}^{3} \\
\mathrm{Q}_{0}=\text { Initial covariance of error } & =1 \mathrm{ft}^{2} \\
\mathrm{~V}_{\mathrm{x}}=\text { Measurement noise intensity } & =1,10,100 \mathrm{ft}^{2} .
\end{array}
$$

By letting $r_{1 p}<r_{1 e}$, we guarantee the possibility of the interception by satisfying the relative controllability requirement discussed in the previous sections. Figure 7.3 shows the feedback gains $K_{p}, L_{p}$, and $K_{e}$ versus time. As seen, all gains become zero at $\mathrm{t}=\mathrm{t}_{\mathrm{f}}=10$ seconds. This implies a zero terminal miss (the occurrence of the interception) by referring to the Equation (7.11), see Figure 7.4. All curves in Figure 7.3 show that the gains are independent of measurement noise intensity, $\mathrm{V}_{\mathrm{x}}$. By introducing Equations (7.31), (7.41) and (7.42), of course $\mathrm{K}_{\mathrm{p}}$ and $\mathrm{K}_{e}$ are independent of $V_{x}$, but for this example, we have found that $L_{p}$ which is actually a function of $V_{x}$ was also insensitive to the variations of $\mathrm{V}_{\mathrm{x}}$. 

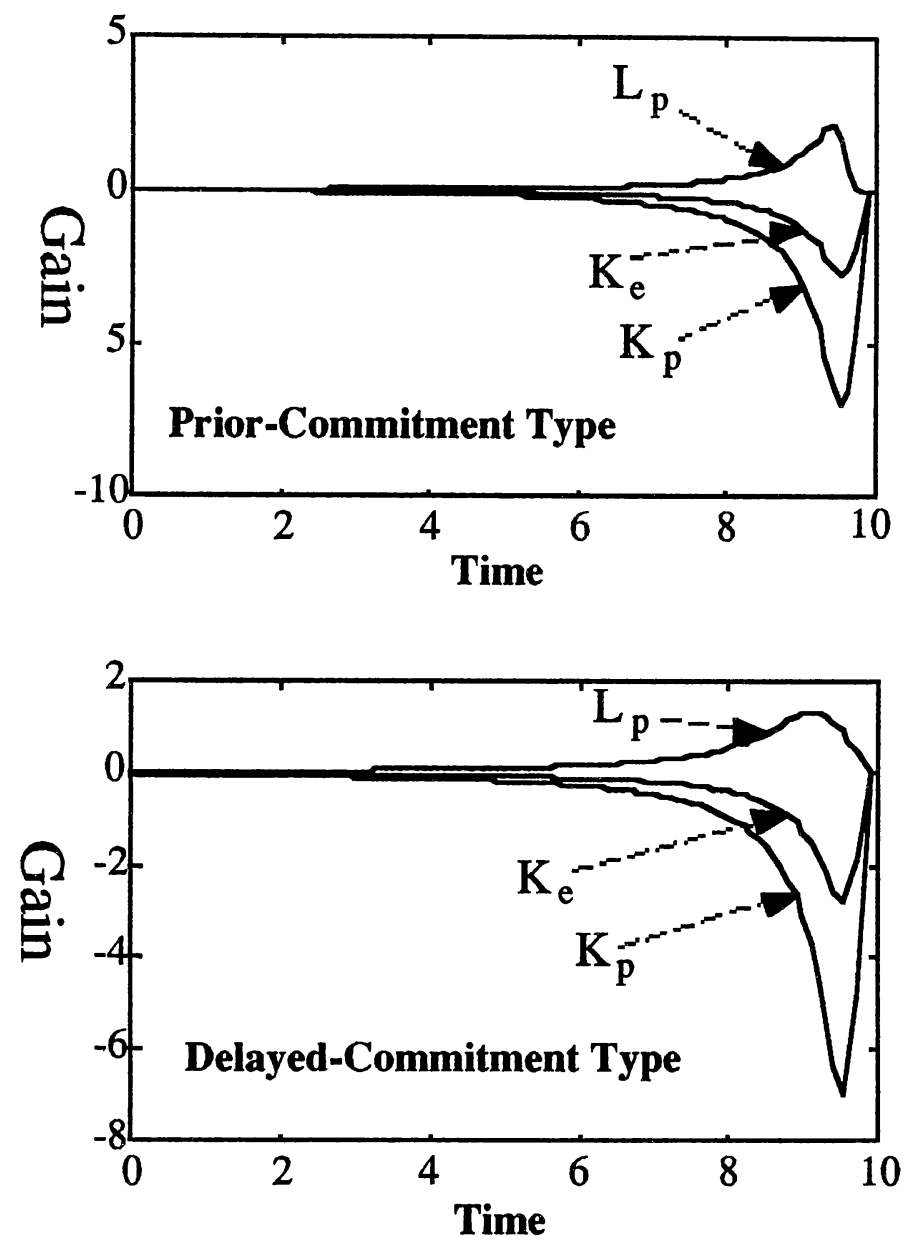

Figure 7.3. The Feedback Strategy Gains

Figure 7.5 shows the estimation error variance of the evader versus time for the same values of $V_{x}$. The top path represents the error variance of the evader, Q11, in the prior-commitment game and the bottom path represents that of the delayed-commitment 
game. As indicated in Figure 7.5, first, the error variances of the priorcommitment and delayed-commitment are almost the same and secondly, by increasing $\mathrm{V}_{\mathrm{x}}$ the corresponding error variance curves become less distinguishable.

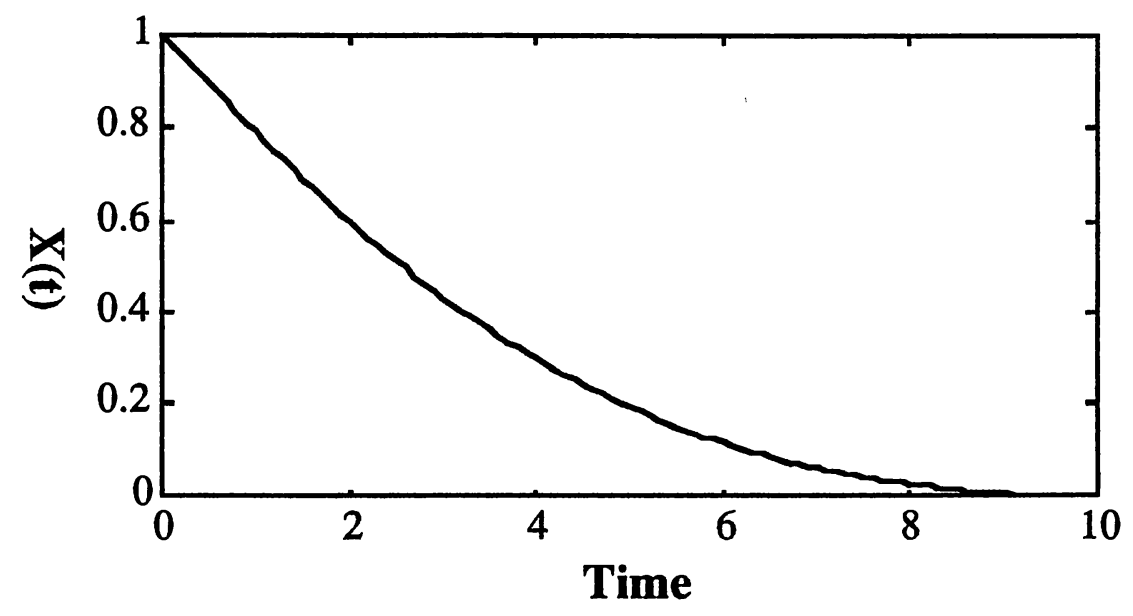

Figure 7.4. Terminal Miss Versus Time, $V_{x}=1$

The curves in Figure 7.5 agree with the fact that 1) the error must become larger as $V_{x}$ increases, i.e. the variance of error will converge to zero slower, 2) with very large $V_{x}$ the control strategies become basically a function of initial estimate $\underline{\hat{x}}(0)$ (see Equations (7.39 \& 7.49f)), i.e. the pursuer can not take advantage of the evader's estimate any more; this fact is illustrated in Figure 7.6. 

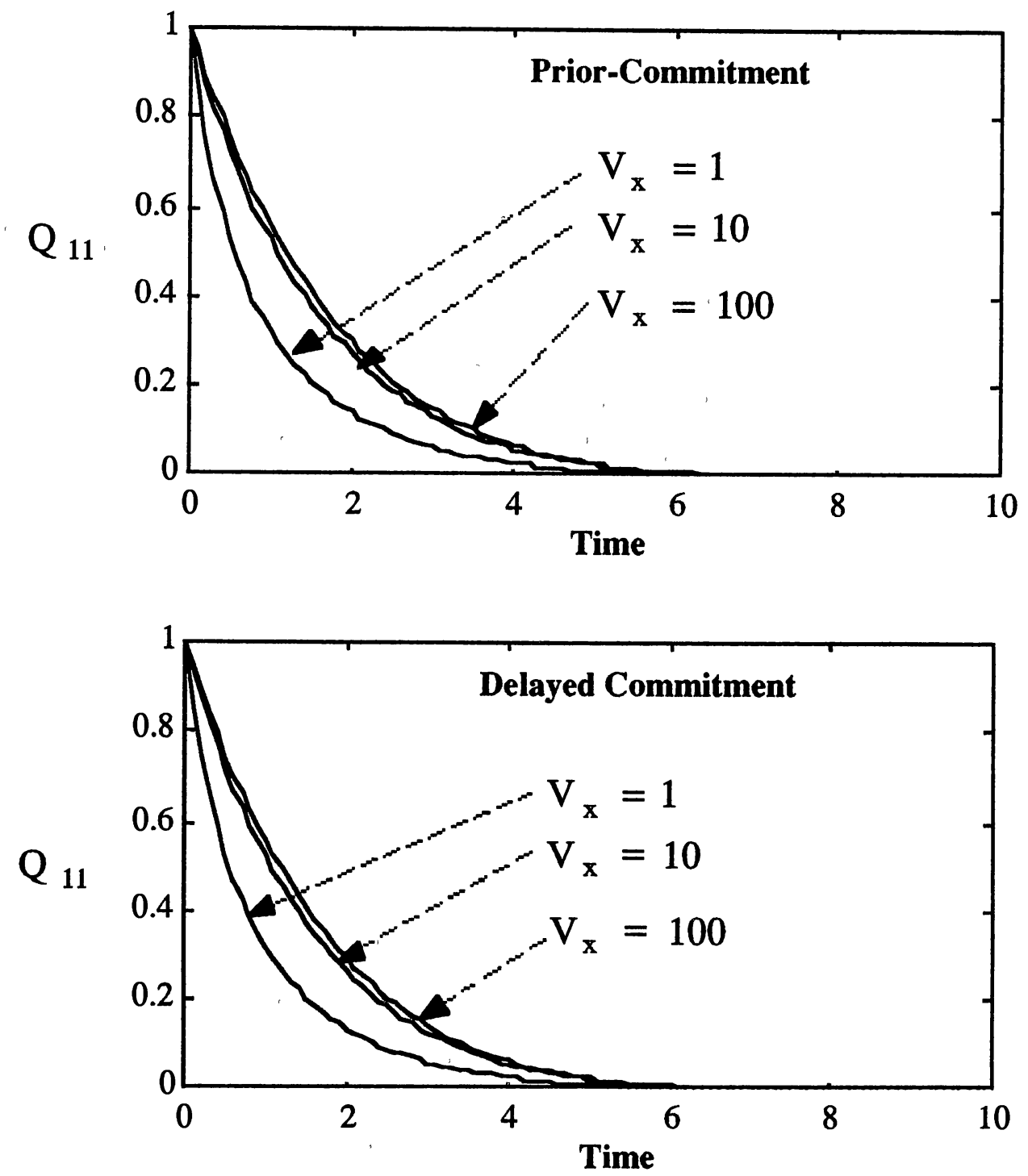

Figure 7.5. The Error Variance of The Evader's Estimate

The relative payoffs* as a function of time for a fixed $V_{x}=100$ are displayed in Figure 7.7. They are always negative which indicate the reduction in capability of the evader. Moreover, the relative payoff

* The relative payoff is the difference between the evader's payoff and the payoff of the perfect information game, and it represents the the direct effect of the evader measurement noise on the payoff of the game. 
associated with the delayed-commitment game is more negative than that of the prior-commitment game. These facts about relative payoffs have been discussed in reference [105] in detail.

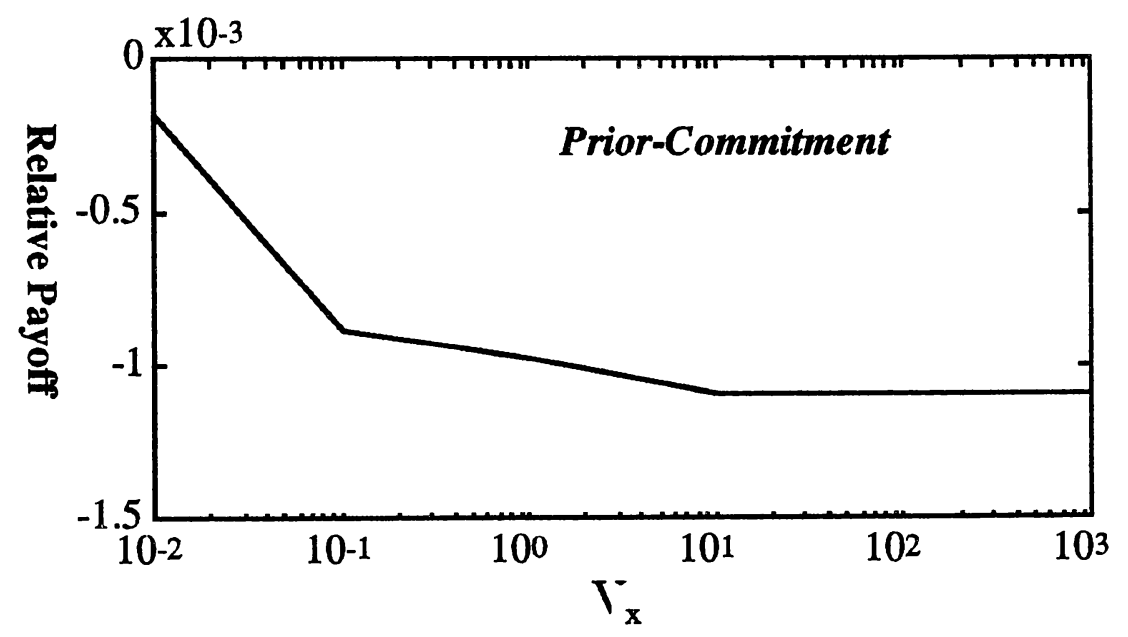

Figure 7.6. The Relative Payoff as a Function of $V_{x}$

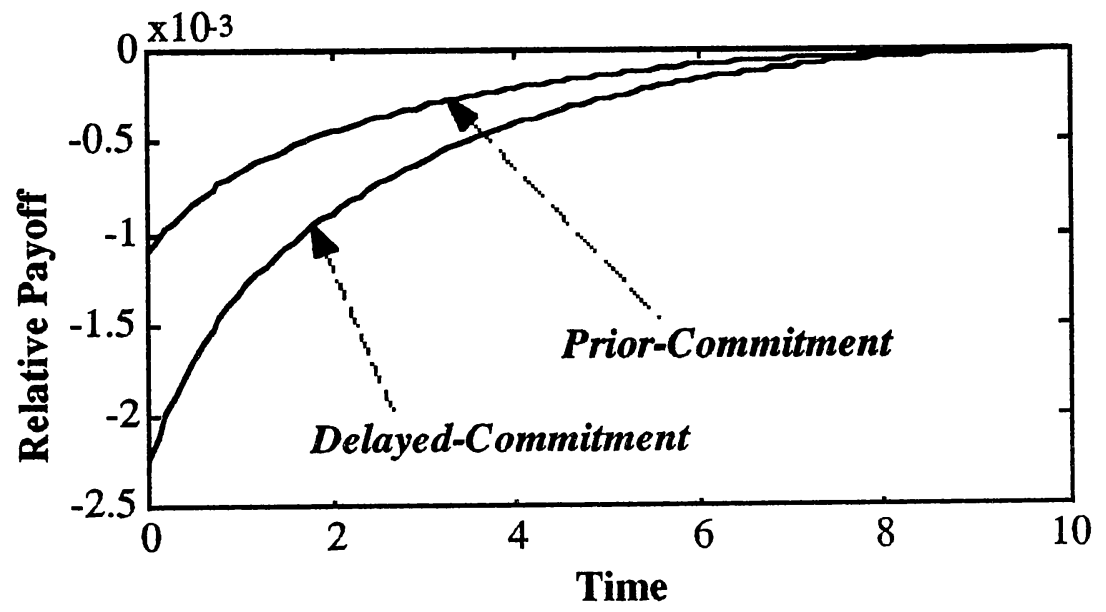

Figure 7.7. The Relative Payoff 


\subsection{Differential Games \& OCM}

In this section we investigate the feasibility of applying differential game theory to modeling of an air-to-air combat task. To do so, we consider the possibility of combination of the optimal control model discussed in chapter IV and pursuit-evasion differential game theory discussed in the previous sections. Because we have seen the applicability of the optimal control tool which can be recognized as a one-sided differential game in manual control systems, it seems logical to consider differential game theory as an analytical tool to analyze the performance of the human operator in an air combat task.

In order to apply the approaches developed in the previous sections, we model human operator as an optimal controller with a lumped time-delay element $\tau$ as its only limitation and observation noise as its remnant. Section 7.6.1 formulates a simple air-to-air combat task. In section 7.6.2 we present a computer simulation of a missile-aircraft problem in which the missile (pursuer) has perfect knowledge of his state as well as that of the aircraft (evader), while the evader, which includs an OCM, is given delayed noise-corrupted information.

\subsubsection{Development of a Simple}

\section{Air-to-Air Combat Task}

In this section we formulate a simple air-to-air problem depicted in Figure 7.8. The top path represents the pursuer and the bottom one represents the evader. Each player is given delayed noise- 
corrupted information about his opponents. The controller 1 and the controller 2 are determined by minimaximizing the payoff performance index of the form

$$
J=E\left\{\begin{array}{l}
\frac{c^{2}}{2} \underline{x}^{\prime}\left(t_{f}\right) \underline{x}\left(t_{f}\right)+ \\
\frac{1}{2} \int_{t_{0}}^{t_{f}} r_{p}^{2} \dot{u}^{2}(t) d t-\frac{1}{2} \int_{t_{0}}^{t_{f}} r_{e}^{2} \dot{v}^{2}(t) d t
\end{array}\right\},
$$

where $\underline{x}($.$) is the predicted terminal miss which shall be defined later.$

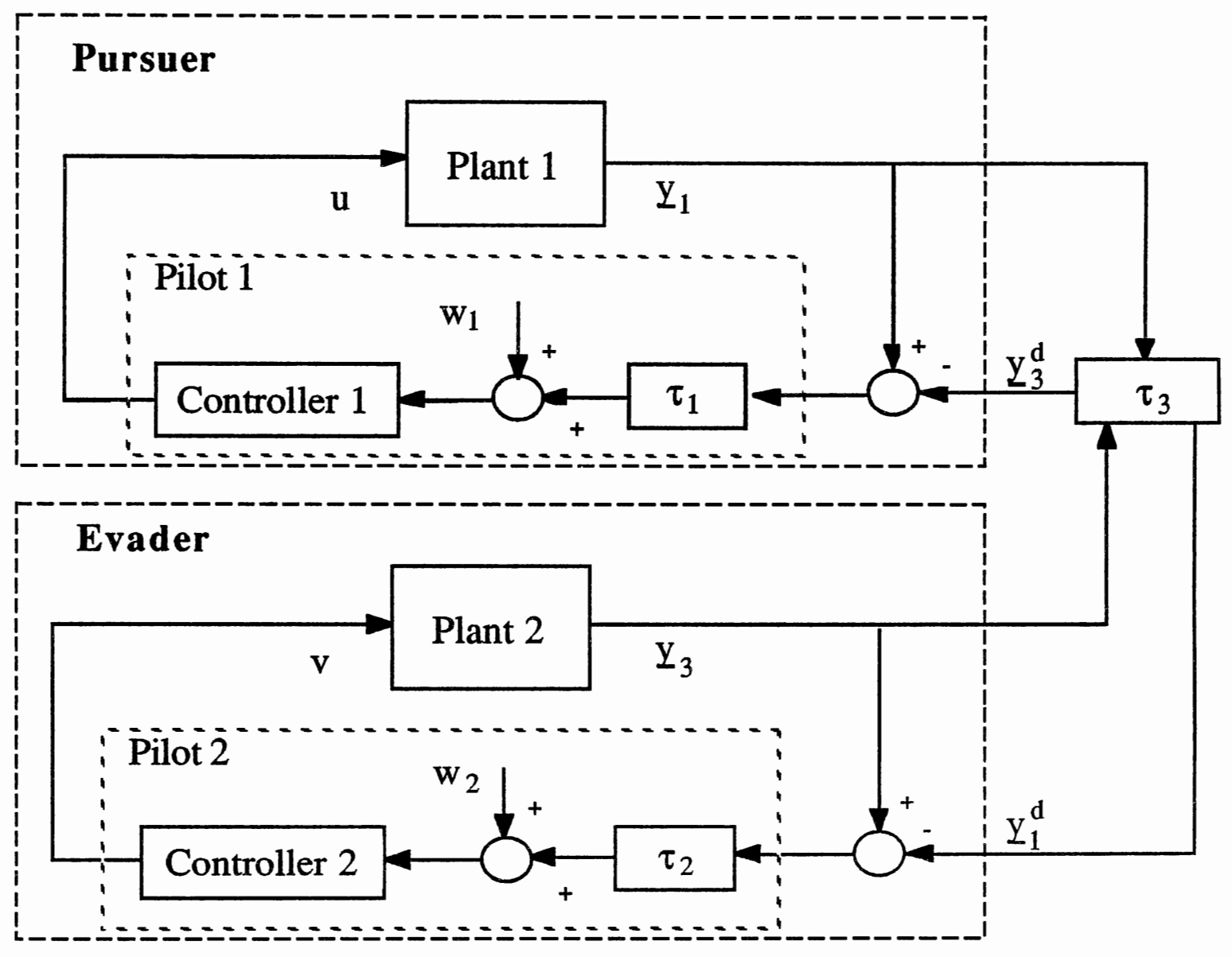

Figure 7.8. Block Diagram of a Simple Air-to-Air Combat Task 
We assume that the operation of the first vehicle (plant1) is described by the following linear equations

$$
\begin{aligned}
& \underline{\dot{x}}_{1}(t)=A_{1} \underline{x}_{1}(t)+\underline{b}_{1} u_{d_{1}}(t) \\
& \underline{y}_{1}(t)=C_{1} \underline{x}_{1}(t),
\end{aligned}
$$

where $\underline{x}_{1}(t)$ is an $n$-dimensional state vector and $y_{1}(t)$ is an $m$ dimensional output (interested state) vector. It is assumed that we have the following Pade approximant for the time-delay $\tau_{1}$,

$$
\begin{aligned}
& \underline{\dot{x}}_{d_{1}}(t)=A_{d_{1}} \underline{x}_{d_{1}}(t)+B_{d_{1}} u(t) \\
& u_{d_{1}}(t)=C_{d_{1}} \underline{x}_{d_{1}}(t)+\underline{d}_{d_{1}} u(t)
\end{aligned}
$$

where $\underline{x}_{d_{1}}$ is a p-dimensional state vector.

By letting $\underline{x}_{2}(t)=\left[\begin{array}{ll}\underline{x}_{1} & \underline{x}_{d_{1}}\end{array}\right]^{\prime}$, from equations (7.54)-(7.58) we have

$$
\begin{aligned}
& \underline{\dot{x}}_{2}(t)=A_{2} \underline{x}_{2}(t)+\underline{b}_{2} u \\
& \underline{y}_{2}(t)=C_{2} \underline{x}_{2}(t),
\end{aligned}
$$

where

$$
A_{2}=\left[\begin{array}{cc}
A_{1} & \underline{b}_{1} c_{d_{1}} \\
0_{p \times n} & A_{d_{1}}
\end{array}\right], \underline{b}_{2}=\left[\begin{array}{c}
\underline{b}_{1} d_{d_{1}} \\
b_{d_{1}}
\end{array}\right] \text { and } C_{2}=\left[\begin{array}{ll}
C_{1} & 0_{m \times p}
\end{array}\right]
$$

Let us assume that the following linear equations define the dynamic of the second vehicle (plant 2),

$$
\begin{aligned}
& \underline{\dot{x}}_{3}(t)=A_{3} \underline{x}_{3}(t)+\underline{b}_{3} v_{d_{1}}(t) \\
& \underline{y}_{3}(t)=C_{3} \underline{x}_{3}(t),
\end{aligned}
$$

where $\underline{x}_{3}(t)$ is an $n$-dimensional state vector and $y_{3}$ is an m-dimensional output vector. The second internal delay $\tau_{2}$ is modeled by the 
Pade approximant

$$
\begin{aligned}
& \underline{\dot{x}}_{d_{2}}(t)=A_{d_{2}} \underline{x}_{d_{2}}(t)+B_{d_{2}} v(t) \\
& v_{d_{1}}(t)=C_{d_{2}} \underline{x}_{d_{2}}(t)+\underline{d}_{d_{2}} v(t),
\end{aligned}
$$

where $\underline{x}_{d_{2}}(t)$ is a p-dimensional state vector.

Let

$$
\underline{x}_{4}(t)=\left[\begin{array}{c}
\underline{x}_{3}(t) \\
\underline{x}_{d_{2}}(t)
\end{array}\right]
$$

The augmented state $\underline{x}_{4}$, satisfies the following augmented equation

$$
\begin{aligned}
& \underline{\dot{x}}_{4}(t)=A_{4} \underline{x}_{4}(t)+\underline{b}_{4} v \\
& \underline{y}_{3}(t)=C_{4} \underline{x}_{4}(t),
\end{aligned}
$$

where

$$
A_{4}=\left[\begin{array}{cc}
A_{3} & \underline{b}_{3} c_{d_{2}} \\
0_{p \times n} & A_{d_{2}}
\end{array}\right], \underline{b}_{4}=\left[\begin{array}{c}
\underline{b}_{3} d_{d_{2}} \\
b_{d_{2}}
\end{array}\right]
$$

and

$$
\mathrm{C}_{4}=\left[\begin{array}{ll}
\mathrm{C}_{1} & 0_{\mathrm{m} \times \mathrm{p}}
\end{array}\right]
$$

Further, we assume that the following equations model the intrasimulator delay $\mathbf{t} 3$,

$$
\begin{aligned}
& \underline{\dot{x}}_{d_{3}}=A_{d_{3}} \underline{x}_{d_{3}}+\underline{b}_{d_{3}} u_{\tau_{3}} \\
& y_{d_{3}}=C_{d_{3}} \underline{x}_{d_{3}}+d_{d_{3}} u_{\tau_{3}}
\end{aligned}
$$

where $\underline{x}_{d_{3}}$ is a $p$-dimensional state vector.

In the light of the above augmented state equations and equations (7.66)-(7.67), we wish to convert our original problem to an equivalent pursuit-evasion problem. This can be done as follows. 


\subsubsection{Evader Development}

Letting

$$
\underline{x}_{5}(t)=\left[\begin{array}{c}
\underline{x}_{4}(t) \\
\underline{x}_{D_{3}}(t)
\end{array}\right]
$$

we can write the following equations

$$
\begin{aligned}
& \underline{\dot{x}}_{5}(t)=A_{5} \underline{x}_{5}(t)+\underline{b}_{5} v, \\
& \underline{y}_{3}(t)=C_{5} \underline{x}_{5}(t), \\
& \underline{y}_{3}^{d}(t)=\left[\begin{array}{ll}
D_{d_{3}} C_{4} & C_{D_{3}}
\end{array}\right] \underline{x}_{5}(t),
\end{aligned}
$$

where

$$
A_{5}=\left[\begin{array}{cc}
A_{4} & 0_{n \times m p} \\
B_{D_{3}} C_{4} & A_{D_{3}}
\end{array}\right], \underline{b}_{5}=\left[\begin{array}{c}
\underline{b}_{4} \\
0_{m p \times 1}
\end{array}\right], C_{5}=\left[\begin{array}{ll}
C_{4} & 0_{m \times m p}
\end{array}\right],
$$

and

$\underline{\mathrm{x}}_{\mathrm{D}_{3}}($.$) is an augmented state vector of dimension \mathrm{m}^{*} \mathrm{p}$ with consisting of $\underline{x}_{d_{3}}($.$) repeated m$ times, $A_{D_{3}}, B_{D_{3}}, C_{D_{3}}$ and $D_{d_{3}}$ are block diagonal matrices with $A_{d_{3}}, \underline{b}_{d_{3}}, c_{d_{3}}$ and $d_{d_{3}}$ as their diagonal elements, respectively (see Chapter V). Finally, by augmenting $\underline{x}_{5}$ with $v$ and letting $\dot{v}(t)=u_{e}(t)$, we can have the following equations describing the evader,

$$
\begin{aligned}
& \underline{\dot{x}}_{e}(t)=A_{e} \underline{x}_{e}(t)+\underline{b}_{e} u_{e}(t), \\
& \underline{y}_{e}(t)=C_{e} \underline{x}_{e}(t), \\
& \underline{y}_{e}^{d}(t)=H_{e} \underline{x}_{e}(t),
\end{aligned}
$$

where 


$$
\underline{x}_{e}(t)=\left[\begin{array}{c}
\underline{x}_{5}(t) \\
v(t)
\end{array}\right], A_{e}=\left[\begin{array}{cc}
A_{5} & \underline{b}_{5} \\
0_{1 \times(n+m p+p)} & 0
\end{array}\right]
$$

and

$$
\underline{\mathrm{b}}_{\mathrm{e}}=\left[\begin{array}{c}
0_{(\mathrm{n}+\mathrm{mp}+\mathrm{p}) \times 1} \\
1
\end{array}\right], \mathrm{C}_{\mathrm{e}}=\left[\begin{array}{ll}
\mathrm{C}_{5} & 0
\end{array}\right], \mathrm{H}_{\mathrm{e}}=\left[\begin{array}{lll}
\mathrm{D}_{\mathrm{d}_{3}} \mathrm{C}_{4} & \mathrm{C}_{\mathrm{D}_{3}} & 0
\end{array}\right] .
$$

The evader is given the information of the form

$$
\begin{aligned}
& \underline{z}_{\mathrm{e} 1}(\mathrm{t})=\underline{y}_{1}^{\mathrm{d}}(\mathrm{t})+\underline{\mathrm{w}}_{\mathrm{e} 1}(\mathrm{t}) \\
& \underline{\mathrm{z}}_{\mathrm{e} 2}(\mathrm{t})=\underline{\mathrm{y}}_{\mathrm{e}}(\mathrm{t})+\underline{\mathrm{w}}_{\mathrm{e} 2}(\mathrm{t}),
\end{aligned}
$$

where $\underline{w}_{e 1}(t)$ and $\underline{w}_{e 2}(t)$ are independent additive white noise processes.

\subsubsection{Pursuer Development}

By letting

$$
\underline{\mathrm{x}}_{6}(\mathrm{t})=\left[\begin{array}{c}
\underline{\mathrm{x}}_{2}(\mathrm{t}) \\
\underline{\mathrm{x}}_{\mathrm{D}_{3}}(\mathrm{t})
\end{array}\right]
$$

and using equations (7.59)-(7.61), we may write

$$
\begin{aligned}
& \underline{\dot{x}}_{6}(t)=A_{6} \underline{x}_{6}(t)+\underline{b}_{6} u, \\
& \underline{y}_{1}(t)=C_{6} \underline{x}_{6}(t), \\
& \underline{y}_{1}^{d}(t)=\left[\begin{array}{ll}
D_{d_{3}} C_{2} & C_{D_{3}}
\end{array}\right] \underline{x}_{6}(t),
\end{aligned}
$$

where

$$
\mathrm{A}_{6}=\left[\begin{array}{cc}
\mathrm{A}_{2} & 0_{\mathrm{n} \times \mathrm{mp}} \\
\underline{b}_{d_{3}} \mathrm{C}_{2} & \mathrm{~A}_{\mathrm{D}_{3}}
\end{array}\right], \underline{\mathrm{b}}_{5}=\left[\begin{array}{c}
\underline{\mathrm{b}}_{2} \\
0_{\mathrm{mp} \times 1}
\end{array}\right], \mathrm{C}_{6}=\left[\begin{array}{ll}
\mathrm{C}_{2} & 0_{\mathrm{m} \times \mathrm{mp}}
\end{array}\right]
$$


By defining the augmented state vector

$$
\underline{x}_{p}(t)=\left[\begin{array}{c}
\underline{x}_{6}(t) \\
u(t)
\end{array}\right]
$$

and letting $\dot{\mathrm{u}}(\mathrm{t})=\mathrm{u}_{\mathrm{p}}(\mathrm{t})$, we can write the pursuer equations as:

$$
\begin{aligned}
& \underline{\dot{x}}_{p}(t)=A_{p} \underline{x}_{p}(t)+\underline{b}_{p} u_{p}(t) \\
& \underline{y}_{p}(t)=C_{p} \underline{x}_{p}(t) \\
& \underline{y}_{p}^{d}(t)=H_{p} \underline{x}_{p}(t)
\end{aligned}
$$

where

$$
A_{p}=\left[\begin{array}{cc}
A_{6} & \underline{b}_{6} \\
0_{1 \times(n+m p+p)} & 0
\end{array}\right], \underline{b}_{p}=\left[\begin{array}{c}
0_{(n+m p+p) \times 1} \\
1
\end{array}\right]
$$

and

$$
\mathrm{C}_{\mathrm{p}}=\left[\begin{array}{ll}
\mathrm{C}_{6} & 0
\end{array}\right], \mathrm{H}_{\mathrm{p}}=\left[\begin{array}{llll}
\mathrm{D}_{\mathrm{d}_{3}} \mathrm{C}_{2} & \mathrm{C}_{\mathrm{D}_{3}} & 0
\end{array}\right]
$$

The pursuer has access to the following information

$$
\begin{aligned}
& \underline{z}_{p 1}(t)=\underline{y}_{e}^{d}(t)+\underline{w}_{p 1}(t), \\
& \underline{z}_{p 2}(t)=\underline{y}_{p}(t)+\underline{w}_{p 2}(t),
\end{aligned}
$$

where noise processes $\underline{w}_{p 1}(t)$ and $\underline{w}_{p 2}(t)$ are independent and white.

\subsubsection{Development of the Pursuer-Evader System}

In order to be able to apply the method discussed in section 7.4.4 (delayed-Commitment solution), we need to develop a stochastic pursuer-evader system. To accomplish that, we define the predicted terminal miss as: 


$$
\underline{x}(t)=C_{p} \Phi_{p}\left(t_{f}, t\right) \underline{x}_{p}(t)-C_{e} \Phi_{e}\left(t_{f}, t\right) \underline{x}_{e}(t),
$$

where

$$
\Phi_{p}\left(t_{f}, t\right)=e^{A_{p}\left(t_{f}-t\right)} \text { and } \Phi_{e}\left(t_{f}, t\right)=e^{A_{e}\left(t_{f}-t\right)}
$$

By differentiating both sides of (7.80) and using (7.68) \& (7.74), as discussed in section 7.3 , we can obtain the following differential equation which describes the dynamics of the pursuit-evasion system

$$
\underline{\dot{x}}(t)=P\left(t_{f}, t\right) u_{p}(t)-E\left(t_{f}, t\right) u_{e}(t)
$$

where

$$
\mathrm{P}\left(\mathrm{t}_{\mathrm{f}}, \mathrm{t}\right)=\mathrm{C}_{\mathrm{p}} \Phi_{\mathrm{p}}\left(\mathrm{t}_{\mathrm{f}}, \mathrm{t}\right) \underline{\mathrm{b}}_{\mathrm{p}} \text { and } \mathrm{E}\left(\mathrm{t}_{\mathrm{f}}, \mathrm{t}\right)=\mathrm{C}_{\mathrm{e}} \Phi_{\mathrm{e}}\left(\mathrm{t}_{\mathrm{f}}, \mathrm{t}\right) \underline{\mathrm{b}}_{\mathrm{e}} \text {. }
$$

In order to convert the measurements of the two players to measurements on terminal miss $\underline{x}(t)$, we may define

$$
\begin{aligned}
& \underline{z}_{p}(t) \equiv C_{p} \Phi_{p} C_{p}^{\#} \underline{z}_{p 1}(t)-C_{e} \Phi_{e} C_{e}^{\#} \underline{z}_{p 2}(t), \\
& \underline{z}_{e}(t) \equiv C_{p} \Phi_{p} C_{p}^{\#} \underline{z}_{e}(t)-C_{e} \Phi_{e} C_{e}^{\#} \underline{z}_{e}(t),
\end{aligned}
$$

where $\underline{z}_{\mathrm{e} 1}(t), \underline{\mathrm{z}}_{\mathrm{e} 2}(\mathrm{t})$ and $\underline{\mathrm{z}}_{\mathrm{p} 1}(\mathrm{t}), \underline{\mathrm{z}}_{\mathrm{p} 2}(\mathrm{t})$ are defined in equations (7.72-7.73) and (7.78-7.79), respectively and $L^{\#}$ denotes the general pseudo-inverse of $\mathrm{L}$. The equations (7.82) \& (7.83) can be rewritten

$$
\begin{aligned}
& \underline{\mathrm{z}}_{\mathrm{p}}(\mathrm{t})=\underline{\mathrm{x}}(\mathrm{t})+\underline{\mathrm{w}}_{\mathrm{p}}(\mathrm{t}), \\
& \underline{\mathrm{z}}_{\mathrm{e}}(\mathrm{t})=\underline{\mathrm{x}}(\mathrm{t})+\underline{\mathrm{w}}_{\mathrm{e}}(\mathrm{t}),
\end{aligned}
$$

where 


$$
\underline{w}_{p}(t)=C_{p} \Phi_{p} C_{p}^{\#} \underline{w}_{p 1}(t)-C_{e} \Phi_{e} C_{e}^{\#} \underline{w}_{p 2}
$$

and

$$
\underline{w}_{e}(t)=C_{p} \Phi_{p} C_{p}^{\#} \underline{w}_{e 1}(t)-C_{e} \Phi_{e} C_{e}^{\#} \underline{w}_{e}(t)
$$

Now, we have converted our original air-to-air combat problem to the stochastic pursuit-evasion problem which is defined by equations (7.81), (7.84) and (7.85) with the payoff index

$$
J=E\left\{\begin{array}{l}
\frac{c^{2}}{2} \underline{x}^{\prime}\left(t_{f}\right) \underline{x}\left(t_{f}\right)+ \\
\frac{1}{2} \int_{t_{0}}^{t_{f}} r_{p}^{2} u_{p}^{2}(t) d t-\frac{1}{2} \int_{t_{0}}^{t_{f}} r_{e}^{2} u_{e}^{2}(t) d t
\end{array}\right\} .
$$

This problem was discussed in section 7.4. The next section illuminates the above results by analyzing a simple missile-aircraft problem in which the evader contains the OCM and the pursuer has perfect information of the game.

\subsubsection{A Missile-Aircraft Example}

This section presents a computer implementation of a missileaircraft problem taken from [106] in which the missile (pursuer) has perfect knowledge of his state as well as that of the aircraft (evader), while the evader including an OCM is given noise-corrupted information. The operation of the minimizer player (missile) is described by

$$
\underline{\dot{x}}_{1}(t)=\left[\begin{array}{ccc}
0 & 1 & 0 \\
0 & 0 & k_{1} \\
0 & 0 & -1 / \tau_{1}
\end{array}\right] \underline{x}_{1}(t)+\left[\begin{array}{c}
0 \\
0 \\
1 / \tau_{1}
\end{array}\right] u_{p},
$$




$$
\mathrm{y}_{1}(\mathrm{t})=\left[\begin{array}{lll}
1 & 0 & 0
\end{array}\right] \underline{\mathrm{x}}_{3}(\mathrm{t})
$$

where $k_{1}$ is a constant number depending on the initial angle of velocity of the missile and $\tau_{1}$ is the missile time constant. For the maxim-izer player (aircraft), the following equations are obtained

$$
\begin{aligned}
& \underline{\underline{x}}_{3}(t)=\left[\begin{array}{ccc}
0 & 1 & 0 \\
0 & 0 & \mathbf{k}_{2} \\
0 & 0 & -1 / \tau_{2}
\end{array}\right] \underline{x}_{3}(t)+\left[\begin{array}{c}
0 \\
0 \\
1 / \tau_{2}
\end{array}\right] v, \\
& \mathrm{y}_{3}(t)=\left[\begin{array}{lll}
1 & 0 & 0
\end{array}\right] \underline{x}_{3}(t),
\end{aligned}
$$

where $k_{2}$ is a constant number depending on the initial angle of velocity of the aircraft and $\tau_{2}$ is the aircraft time constant. The equations (7.88) and (7.90) give the position of the missile and the aircraft, res-pectively. Further, the payoff index is given by

$$
J=E\left\{\begin{array}{l}
\frac{c^{2}}{2}\left(y_{1}\left(t_{f}\right)-y_{3}\left(t_{f}\right)\right)^{2}+ \\
\frac{1}{2} \int_{t_{0}}^{t_{f}} r_{p}^{2} u_{p}^{2}(t) d t-\frac{1}{2} \int_{t_{0}}^{t_{f}} r_{e}^{2} \dot{v}^{2}(t) d t
\end{array}\right\} .
$$

A general class of this problem was formulated in the previous section. We solved this problem for the following constants and parameters listed bellow,

$$
\begin{array}{ll}
\mathbf{t}_{\mathrm{f}}=\text { Final time } & =2 \mathrm{sec} . \\
\mathrm{t}_{0}=\text { Initial time } & =0 \mathrm{sec} . \\
\mathrm{c}^{2}=\text { Terminal miss factor } & =0.04 \mathrm{ft}^{-2} \\
\mathbf{r}_{\mathrm{p}}=\text { Missile control factor } & =0.1\left(\mathrm{ft}^{-2} \mathrm{sec} .^{3}\right) \\
\mathbf{r}_{\mathrm{e}}=\text { Airplane control factor } & =10\left(\mathrm{ft}^{-2} \mathrm{sec} .{ }^{3}\right)
\end{array}
$$


$\tau_{1}=$ Missile time constant

$\tau_{2}=$ Aircraft time constant

$\mathrm{Q}_{0}=$ Initial error variance

$\mathrm{W}_{\mathrm{e}}=$ Evader measurement noise variance
$=1 \mathrm{sec}$.

$=1 \mathrm{sec}$.

$=1 \mathrm{ft}^{2}$

$=10 \mathrm{ft}^{2}$.

Figure 7.9 shows the effect of the intersimulator delay $\tau_{3}$ on the overall system performance measured by the covariance of the terminal miss given the measurements of the evader, $\mathrm{X}\left(\mathrm{t}_{\mathrm{f}}\right)$. This is actually, the mean square of terminal miss and it can be viewed as the mean square actual error between the position of the missile and the aircraft. As indicated in the figure $7.8, \mathrm{X}\left(\mathrm{t}_{\mathrm{f}}\right)$ initially increases and will reach a maximum before decreasing. This is exactly the same interesting trend which is observed for the formation flight task (see Chapter VI). This results could be expected to be in full agreement with our previous results obtained in the formation flight problem, because the differential game is actually a two-sided optimal control theory.

In addition, it seems that the internal delay $\tau_{2}$ does not have significant effect on mean square of the terminal miss, $\mathrm{X}\left(\mathrm{t}_{\mathrm{f}}\right)$, especially for larger $\tau_{3}$. The effect of the internal delay on $X(t)$ is shown in Figure 7.10. As seen, $X(t)$ will increase with $\tau_{2}$, however the amount of increase is not significant. 

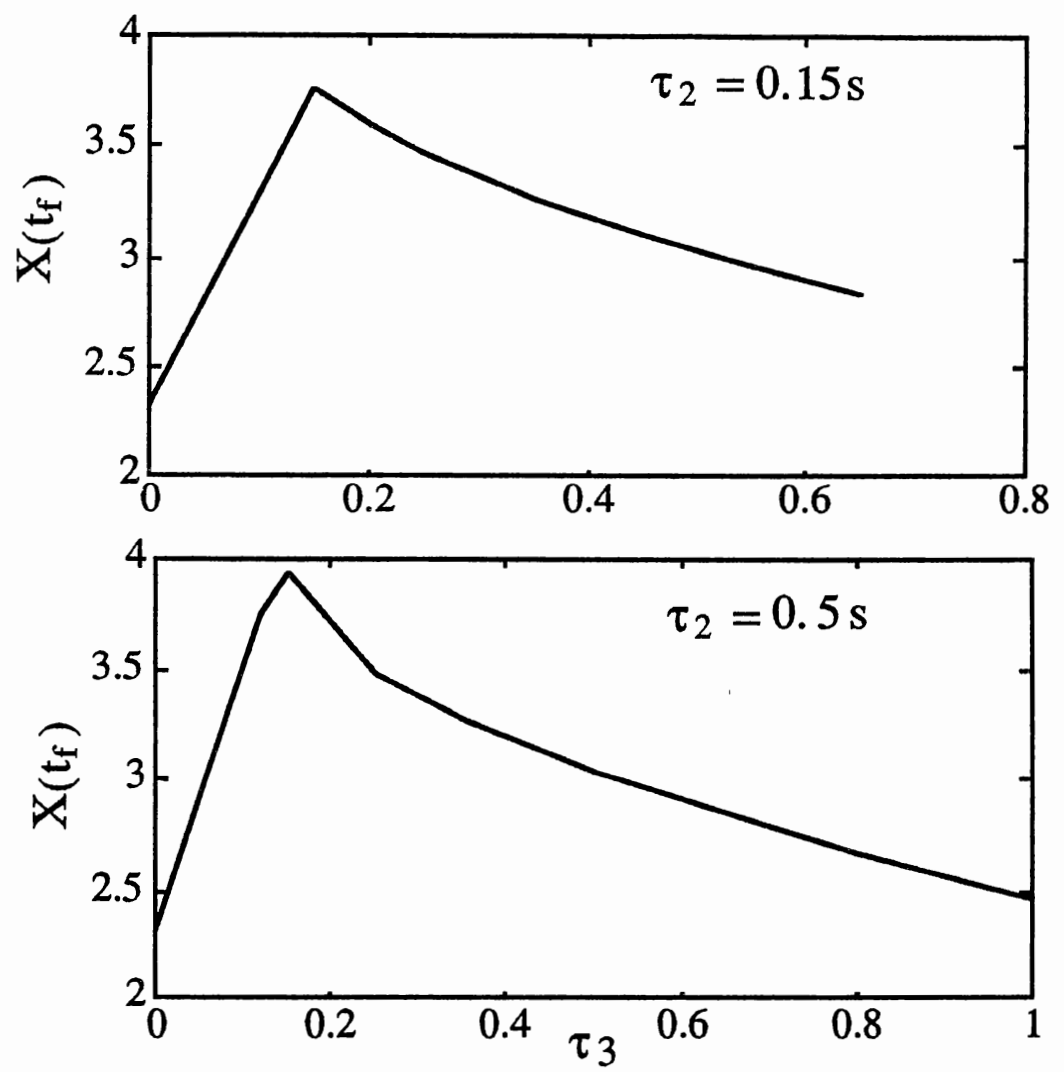

Figure 7.9. The Effect of $\tau_{3}$ on a Missile-Aircraft Problem

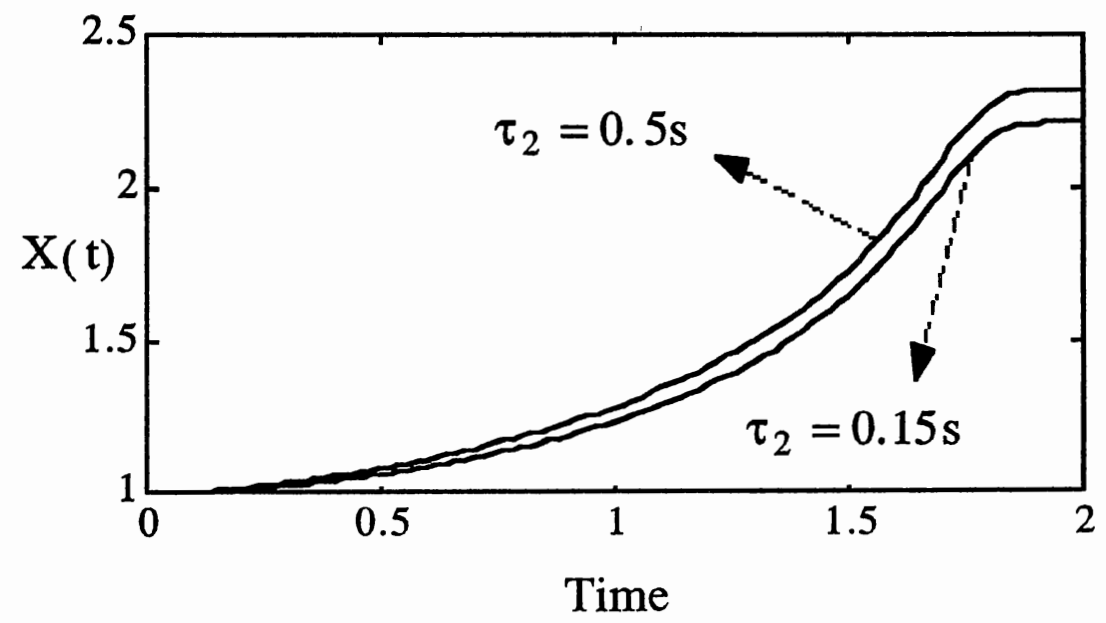

Figure 7.10. The Effect of $\tau_{2}$ on Missile-Aircraft Problem, $\tau_{3}=0$. 


\subsection{Summary}

In this chapter we first introduced air-to-air combat tasks which can be considered as the game of combat, and they may be formulated as pursuit-evasion differential games. Sections 7.2 through 7.4 present a review of literature on differential game theory and introduce the pursuit-evasion problems. Section 7.2 presented a general view of differential game theory. Pursuit-evasion problems have been discussed in sections 7.3 and 7.4 .

In section 7.5, we applied the methods reviewed in the previous sections to a classical interception problem as an example.of a stochastic pursuit-evasion game. In section 7.6, by applying pursuitevasion differential game theory, we first developed a model for a simple air-to-air combat task and then we analyzed a missile-aircraft problem in which the aircraft (evader) is given noisy delayed information. We observe that the mean square terminal miss, as a function of intersimulator delay, is nonomotonic; it initially increases and will reach a maximum before decreasing. This was exactly the same trend that has been observed for formation flight task problems discussed in Chapter VI. 
CHAPTER VIII

\section{CONCLUDING REMARKS}

\subsection{Conclusions}

Time delays can cause significant problems in the simulation of highly responsive systems, such as fighter aircraft. It is very important to analyze the adverse effects of these delays. Several efforts have been made to analyze the effects of delay in single flight simulators, but there has been nothing found in the literature for analysis of the delay in networked flight simulators. This study provides an analytical method to show the effects of certain time delays, in particular inter-simulator delay, in some networked flight simulators.

The first phase of this study has investigated the effect of the length of the inter-simulator delay on pilot performance in a simple formation flight task. For this task there are two aircraft: a lead pilot and a Wingman. The lead pilot is attempting to maintain level flight while experiencing turbulence. The Wingman is trying to follow the lead, and is experiencing the same turbulence.

There were several interesting, and unexpected, results from the analysis of this task. The first was that the tracking error is not a monotonically increasing function of the time delay between the two simulators, as might be expected. The error perceived by the 
Wingman decreases initially as the inter-simulator delay is increased. After reaching a minimum, the error then increases. In addition, the actual error (by-passing the inter-simulator delay) has the opposite pattern. It increases first, then decreases after reaching a maximum.

It was also found that the point at which the perceived error reaches a minimum (as a function of inter-simulator delay) will increase as the intra-simulator delay of the Wingman increases, but will decrease as the intra-simulator delay of the lead pilot increases.

In order to explain these results, several simple optimal tracking problems were analyzed. These studies showed that, indeed, it is the nature of the optimal tracking problem which brings on the phenomena seen in the formation flight task.

As a logical extension, the second phase of this study investigated the feasibility of applying differential game theory to the modeling of an air-to-air combat task by considering the possibility of combination of optimal control model and differential games for a simple task. After introducing a model for a simple air-to-air combat task, we analyzed a missile-aircraft problem in which the aircraft (evader) is given a noisy delayed information. Again, we observed that the mean squared terminal miss, as a function of intersimulator delay, is non-monotonic; it initially increases and will reach a maximum before decreasing. This was exactly the same trend that has been observed for the formation flight task problems discussed in Chapter VI. 


\subsection{Suggested Topics for Further \\ Research}

The previous chapter considered a simple case of a combat game formulated as a zero-sum, two-person pursuit-evasion differential game with the following characteristics:

1. There was a single payoff index of performance, which was a quadratic of the terminal miss and the time of the capture, assigning a unique number (value of the game) to each pair of the strategies of the pursuit-evader game.

2. It was a single target for the combat game.

3. The evader (the maximizer player) had no offensive capabilities; he only strived for maximizing the time of capture from an initial state to the target state. The pursuer attempted to minimize the the time of the capture.

4. The external state disturbances have been neglected.

The pursuer-evader model developed in chapter VII is not accurate for a general air-to-air combat task (Dogfight problem*) in which the evader also has offensive capabilities. However, an encounter between aggressive aircraft may end in a misille-aircraft problem discussed in the previous chapter. For further research, the following extensions are recommended.

* According to the terminology of Isaacs [81]. 


\subsubsection{Noisy-State Pursuit-Evasion}

\section{Differential Games}

Consider the following state-differential equations which define the dynamics of pursuer and evader,

$$
\begin{aligned}
& \underline{\dot{x}}_{p}(t)=A_{p} \underline{x}_{p}(t)+B_{p} \underline{u}_{p}(t)+E_{p} \underline{w}_{p}(t), \\
& \underline{\dot{x}}_{e}(t)=A_{e} \underline{x}_{e}(t)+B_{e} \underline{u}_{e}(t)+E_{e} \underline{w}_{e}(t),
\end{aligned}
$$

where subscripts $\mathrm{p}$ and $\mathrm{e}$ stand for pursuer and evader respectively, and $\underline{w}_{p}$ and $\underline{w}_{e}$ are independent white Gaussian noise. It is assumed that the initial states $\underline{x}_{p}\left(t_{0}\right)$ and $\underline{x}_{e}\left(t_{0}\right)$ are Gaussian white noise vectors, uncorrelated with both state disturbances, and the available measurements for the pursuer and the evader are given in Equations (8.3) and (8.4), respectively,

$$
\begin{aligned}
& \underline{z}_{p p}(t)=C_{p p} \underline{x}_{p}(t)+\underline{v}_{p p}(t), \\
& \underline{z}_{p e}(t)=C{ }_{p e} \underline{x}_{e}(t)+\underline{v}_{p e}(t), \\
& \underline{z}_{e p}(t)=C C_{e p} \underline{x}_{p}(t)+\underline{v}_{e p}(t), \\
& \underline{z}_{e e}(t)=C C_{e e} \underline{x}_{e}(t)+\underline{v}_{e e}(t) .
\end{aligned}
$$

where independent noise processes $\underline{v}_{(.)}$are white, Gaussian, and uncorrelated with initial states and state noises. For the linear systems given in (8.1) through (8.4), the following differential game is defined: Determine a saddle point $\left(\underline{u}_{p}^{*}, \underline{u}_{e}^{*}\right)$ of the payoff criterion of the form 


$$
J\left(\underline{u}_{p}, \underline{u}_{e}\right)=\frac{1}{2} E\left\{\lim _{T \rightarrow \infty} \frac{1}{T}\left[\begin{array}{l}
\underline{x}^{\prime}(T) R_{c} \underline{x}(T)+ \\
\int_{0}^{T}\left[\underline{u}_{p}^{\prime}(t) R_{p} \underline{u}_{p}(t)-\underline{u}_{e}^{\prime}(t) R_{e} \underline{u}_{e}(t)\right] d t
\end{array}\right]\right\}
$$

subject to the constraints given in (7.1) and (7.2), where the matrices $C_{p}$ and $C_{e}$ yielding the interested states, the matrices $R_{c}, R_{p}$ and $R_{e}$ are positive definite, and the terminal miss $\underline{x}(T)$ is of the form

$$
\underline{x}(T)=C_{p} \underline{x}_{p}(T)-C_{e} \underline{x}_{e}(T) .
$$

This is exactly a zero-sum, two person stochastic differential game with the noisy measurements given in equations (7.3) \& (7.4). As discussed in section 7.6 , in order to develop a complete OCM model, which includes the motor noise, for an air-to-air combat task,.the solution of the above general stochastic differential game is necessary. As selected bibliographies, references [103], [105] and [106] are suggested for future study.

\subsubsection{Non-Zero Sum Differential Games}

As mentioned earlier, in real air-to-air combat, each aircraft strives to destroy its encounter aircraft, while ensuring his survival. To be so, before missile launch each aircraft by right maneuvering has to bring its hostile opponent inside its weaponry range so that its missile can reach the opponent. In addition, after missile launch each aircraft must maneuver such that the missile of its opponent will not reach it. Therefore, we may say that the first player (the first aircraft) strives to minimize the distance from its missile to its opponent (the encounter aircraft), $1_{1}$, and to maximize, at the same 
time, the distance from its opponent's missile to its current position, $1_{2}$, while the second player (the second aircraft) behaves the opposite way; he attempts to minimize $1_{2}$ and maximize $1_{1}$. In this case, one may first suggest two different performance indices $J_{1}\left(l_{1}, l_{2}\right)$ and $\mathrm{j}_{2}\left(\mathrm{l}_{1}, \mathrm{l}_{2}\right)$, then determine the optimal strategies (the controllers) by minimaximizing the indices $\mathrm{J}_{1}$ and $\mathrm{J}_{2}$. This is exactly a two-person, non-zero-sum differential game.

Therefore, in order to include the offensive behavior of the evader (the opponent), we may have to consider non-zero sum differential games, since the zero-sum pursuit-evasion differential games emphasize the non-offensive maneuvering of the evader. Further research needs to be done on this kind of game, in particular on the determination of the appropriate choices of the cost indices $J_{1}$ and J2, especially when each player includes an OCM which models the pilot with different subjective performance indices.

References [114] \& [115] analyzed some air-to-air combat problems and addressed the problem of optimal missile firing which is based on the solutions of non-zero sum differential games. For preliminary background about non-zero sum differential games and their properties, References [116-117] are suggested.

\subsubsection{Two-Target Qualitative Games}

As mentioned earlier, chapter 7 concentrated on single-target pursuit-evasion differential games which can be used to modeling "idealized" air-to-air combat problems. In a single-target differential game, there is actually no winner because of the a priori compromise 
between the two players. This is called the role determination [81] problem which is a preassigned roles for the two-players (pursuer versus evader) and its limitations has been discussed in Ref. [118]. Although the role determination problem may be resolved by considering two simultaneous pursuit-evasion games, the two target qualitative games seems to be more promising [113].

In a two-target differential game, each player (each aircraft with offensive missile) strives to bring the state of the game from any given initial condition to its own target set, while not letting his opponent (the encounter aircraft with offensive capabilities) drive the game to his target set. For problem formulation and the determination of the optimal strategies in a two-target qualitative differential games, Refs. [119]-[123] are suggested. 


\section{REFERENCES}

[1] Rolfe, J. M. and K. J. Staples, Flight Simulatiom., New York, Cambridge University Press, 1986.

[2] Levison, W. H., " Application of The Optimal Control Model to The Design of Flight Simulation Experiment.", BBN Lab., Inc., MA., 1985.

[3] Levison, W. H. and A. W. F. Huggins, " Modeling The Effects Of System Delays and Lags on Tracking Performance.", BBN Lab., Inc., MA., 1986.

[4] Levison, W. H. and B. Papazian, "The effects of Time Delay and Simulator Mode on Closed-Loop Pilot/Vehicle Performance: Model Analysis and Manned Simulation Results.", BBN Lab. Inc., MA., 1987.

[5] Johns, J., " A Study of The Efeects of Delay Times in a Dome-toDome Simulation Link.", Northrop Corporation, Aircraft Division, CA., 1988.

[6] Gum, D. R. and E. A. Martin, " The Flight Simulator time Delay Problem.", Aeronautical Systems Division. WrightPatterson Air Force Base, OH 45433

[7] Levison, W. H., " Model-Based Guidlines For Simulator Temporal Fidelity Requirements.", BBN Systems and Technologies Corporation, MA, 1989.

[8] Hagan, M. T., " Effects of Time delays in Networked Simulators.", Final Report, 1990 USAF-UES, AFOSR, 1990.

[9] Johnston, D. L., " Modular Simulators: Is A Local Area Network Sufficient?", Tactical and Training Systems Division., Logicon. Inc., San Diego, CA, 1985. 
[10] Hecker, M., " Modular Simulators: How To Make It Work.", Tactical and Training Systems Division., Logicon, Inc., San Diego, CA, 1985.

[11] Fitts, P.M., " The Information Capacity of The Human Motor System In Controlling The Amplitude of Movement.", Journal of Experiment Psychology, vol. 47, pp. 381-391, 1954.

[12] Sheridan, T. B. and W. R. Ferrel, " Remote Manipulative Control With Transmission Delay.", IEEE Trans. on Human Factors in Electronics, vol. 4, pp. 25-29, 1963.

[13] Smallward, R. D., "Internal Models and The Human Instrument Monitor.", IEEE Trans. on Human Factors in Electronics, vol. 8, No. 3, 1967.

[14] Elkind, J. I., " Characteristics of Simple Manual Control Systems.", M.I.T. Lincoln Lab., Lexington, MA, 1956

[15] McRuer, D. T. and E. S. Krendel, " Dynamic Response of Human Operators.", Wright Air Development Center, Wright Patterson Air Force Base, Dayton, 1957

[16] Adams, J. A., " Human Tracking Behavior.", Psych. Bull., vol. 58, pp. 55-79, 1961.

[17] Bekey, G. A., H. F. Meissing and R. E. Rose, "mathematical Models of Human Operators in Simple Two-Axis Manual Control Systems.", IEEE Trans. on Human Factors in Electronics, vol. 6, pp. 42-52, 1965.

[18] Bates, J. A., " Some Characteristics of a Human Operator.", J. IEE, vol. 94, pt. 11a, pp. 298-304,1947

[19] Belsley, S. E., " Man Machine System Simulation For Flight Vehicles.", IEEE Trans. on Human Factors in Electronics, vol. 4, pp. 4-14, 1963.

[20] Sanders, A. F., " Human Performance Models and System Design.", Department of Psychology, RWTH Aachen, 1989. 
[21] Senders, J. W., " The Human Operator as a Monitor and Controller of Multidegree of Freedom Systems.", IEEE Trans. on Human Factors in Electronics, vol. 5, 1964.

[22] Stassen, H. G. , G. Johannsen and N. Moray, " Internal Representation, Internal Model and Mental Workload.", Automatica, vol. 26, no. 4, pp. 811-820, 1990.

[23] Jury E. I. and T. Pavlidis, " A Literature Survey of Biocontrol Systems.", IEEE Trans. on Automatic Control, vol. 8, pp. 210-217, 1963.

[24] Snyder, D. L. , " A Nonlinear Study of Compensatory Manual Control Systems.", IEEE Trans. on Human Factors in Electronics, vol. 5, pp. 25-28, 1964.

[25] Young, L. R., and L. Stack, " Variable Feedback Experiments Testing a Sampled Data Model or Eye Tracking Movements.", IEEE Trans. on Human Factors in Electronics, vol. 4, pp. 38-51, 1963.

[26] Lang, R. P. and A. G. Vacroux, " Operator Centered Adaptive Compensation of Continuous Manual Control Systems.", IEEE Trans. on Man-Machine Systems, vol. 10, no. 2, 1969.

[27] Baron, S. and D. L. Kleinman, " The human as an Optimal Controller and Information Processor.", IEEE Trans. on Man-Machine systems, vol. 10, no. 1, pp. 9-17,1969.

[28] Pew, R. W. and S. Baron, " Perspectives on Human Performance Modelling.", Automatica, vol. 19, pp. 663-676, 1983.

[29] Sheridan, T. B., " Measuring, Modeling and Augmenting Reliability of Man-Machine Systems.", Automatica, vol. 19, pp. 637-644, 1983.

[30] Margulies, F. and H. Zemanek, " Man's Role in Man-Machine Systems.", Automatica, vol. 19, pp. 677-683, 1983. 
[31] Secarea, V. V., Jr., " Beyond Knobs and Dials: Toward an Intentional Model of Man-Machine Interaction.", IEEE Service Center, Piscataway, NJ., USA, pp. 763-769.

[32] Rasmussen, J., " Skills, Rules, Knowledge: Signals, Signs, and Symbols, and Other Distinction in Human Performance Models.", IEEE Trans. Systems, Man and Cybernetics, vol. 13, no. 3, 1983.

[33] Todosiev, E. P., " Human Performance in a Cross-Coupled Tracking System.", IEEE Trans. on Human Factors in Electronics, vol. 8, 1967.

[34] Miller, D. C. and J. Elkind, " The Adaptive Response of The Human Controller to Sudden Change in Controlled Process Dynamics.", IEEE Trans. on Human Factors in Electronics, vol. 8, no. 3, 1967.

[35] Todosiev, E. P., E. Rose and L. G. Summers, " Human Performance in Single and Two-Axis Tracking Systems.", IEEE Trans. on Human Factors in Electronics, vol. 8, no. 2, 1967.

[36] Wierwille, W., " A Theory For Optimal Deterministic Characterization of Time-Varying Human Operator Dynamics.", IEEE Trans. on Human Factors in Electronics, vol. 4, 1965.

[37] Smith, R. H., "On The Limits of Manual Control.", IEEE Trans. on Human Factors in Electronics, vol. 4, pp. 56-59, 1963.

[38] Costello, R. G. and T. J. Higgins, "an Inclusive Classified Bibliography pertaining to Modeling the Human Operator as an Element in an Automatic Control System.", IEEE Trans. on Human Factors in Electronics, vol. 7, no. 4, 1966.

[39] Elkind, J. I. and C. D. Forgie, " Characteristics of The Human Operator In Simple Manual Control Systems.", IRE Trans. on Automatic Control, vol. 4, pp. 44-55, 1959. 
[40] Sheridan, T. B. and W. R. Ferrell, Man-Machine System., MIT Press, 1974.

[41] McRuer, D. T., " Human Dynamics in Man-Machine Systems.", Automatica, vol. 16, no.3, pp. 237-253, 1980.

[42] Tustin, A. , " The Nature of The Operator's response in Manual Control and It's Implication For Controller design. ", J. IEE, vol. 94, pt. IIA, pp. 190-202, 1947

[43] Wierwille, W. W. , " Improvement of The Human Operator's Tracking Performance by Means of Optimum Filtering and Prediction.", IEEE Trans. on Human Factors in Electronics, vol. 5, pp. 20-24, 1964.

[44] Young, L. R., D. M. Green, J. I. Elkind, and J. A. Kelly, " Adaptive Dynamics Response Characteristics of The Human Operator in Simple Manual Control.", IEEE Trans. on Human Factors in Electronics, vol. 5, pp. 6-13, 1964.

[45] Elkind, J. I. and D. L. Darley, " The Normality of Signals and Describing Function Measurements of Simple Manual Control Systems.", IEEE Trans. on Human Factors in Electronics, vol. 4, pp. 52-55, 1963.

[46] Levison, W. H., S. Baron and D. L. Kleinman, " A Model For_ Human Controller Remnant.", IEEE Trans. on Man-Machine Systems, vol. 10, no. 4, 1969.

[47] Levison, W. H. and J. I. Elkind, " Two-Dimensional Manual Control System With Seperated Displays.", IEEE Trans. on Human Factors in Electronics, vol. 8, pp. 202-209, 1967

[48] Senders, J. W. , " The Hunan Operator as a Monitor and Controller of Multi-Degree Of Freedom Systems.", IEEE Trans. on Human Factors in Electronics, vol. 5, PP. 2-5, 1964.

[49] Kleinman, D. L., S. Baron and W. H. Levison, " An Optimal Control Model Of Human Response Part I: Theory and Validation.", Automatica, vol. 6, pp. 357-369, 1970. 
[50] McRuer, D. and H. R. Jex, " A review of Quasi-Linear Pilot Models.", IEEE Trans. on Human Factors in Electronics, vol.8, pp. 231-249, 1967.

[51] Dander, V. A. , " Predicting Pilot Ratings of Multi-axis Control Tasks From Single axis Data.", IEEE Trans. on Human Factors in Electronics, vol. 4, pp. 15-17, 1963

[52] McRuer, D. T., et.al., " New Approach to Human-pilot Vehicle Dynamics Analysis.", Tech. Rept. AFFDL-TR-67-150, 1968.

[53] McRuer, D. T., D. Graham, E. Krendel and W. Reisener, "Human Pilot Dynamics in Compensatory Systems.", Air ForceFlight Dynamics Lab., Wright-Patterson Air Force Base, Dayton, Ohio, Tech. Rept., AFFDL-TR-65-15, 1965.

[54] Jackson, G. A., " A Method For The Direct Measurement of Cross-Over Model Parameters.", IEEE Trans. on ManMachine Systems, vol. 10, no. 1, 1969.

[55] Young, L. R. and J. L. Merry, " Bang-Bang Aspects of Manual Control in High-order systems.", IEEE Trans. on Automatic Control, vol. 10, pp. 336-341, 1965.

[56] Brogan, W. L. , Modern Control Theory, Second Edition, Prentice-Hall Inc., New Jersy, 1985

[57] Close, C. M. and Friedrick, D. K. , Modeling and Analysis of Dynamic systems., Houghton Miflin Company, Boston, 1978.

[58] Rowland, J. R. , Linear Control Systems, Modeling, Analysis. and Design, John Wiley \& Sons, New York, 1986.

[59] Kirk, D. E., Optimal Control Theory an Introduction, PrenticeHall, Inc., New Jersey, 1970.

[60] Anderson, B. D. O. , and J. B. Moore, Optimal Control, Linear Quadratic Methods., Prentice-Hall, New Jersy, 1990. 
[61] Rosenbrock, H. H., and C. Storey, Mathematics of Dynamical Systems, John Wiley \& Sons, Inc., Newyork, 1970.

[62] Boyce, W. E. and R. C. Diprima, Elementary Differential Equations and Boundary Value Problems, John Wiley \& New York, 1986.

[63] Meditch, J. S., Stochastic Optimal Linear Estimation and Control, McGraw-Hill, NY., 1969.

[64] Stengel, R. F., Stochastic Optimal Control, Theory and Application, John Wiley \& Sons Inc., NY., 1986.

[65] Stein, G. , " Generalized Quadratic Weights For Asymptotic Regulator Properties.", IEEE Trans. on Automatic Control, vol. 24, no. 4, pp. 559-566,1979.

[66] Kalman, R. E. , " When Is a Linear Control System Optimal?", Transactions, ASME ser. D:J. Basic Eng. vol. 86, pp. 110,1964 .

[67] Kwakernaak, H. and R. Sivan, Linear Optimal Control Systems, Wiley-Interscience, New York, 1972.

[68] Shinners, S., Control System Design, John Wiley \& Sons Inc., NY.

[69] Pattiputi, K. R. and D. L. Kleinman, " A Dynamic Decision Model of Human Task Selection Performance.", IEEE Trans. on Systems and Cybernetics, vol. 13, no. 3, 1983.

[70] Kleinman, D. L. , " Optimal Control of Linear Systems With Time-Delay and Observation Noise.", IEEE Trans. on Automatic Control, 1969.

[71] Phatak, A. V. and K. M Kessler, "Modeling the Human Guner in an Anti-Aircraft Artilery (AAA) Tracking Task.", Human Factors, pp. 477-494, 1977. 
[72] Phatak, A., H. Weinert, I. Segal and C. N. Day, " Identification of a Modified Optimal Control Model for the Human Operator.", Automatica, pp. 31-41, 1976.

[73] Shamash, Y., " Linear System Reduction Using Pade Approximation to allow retention of dominant modes.", Int. J. Control, vol. 21, no. 2, pp. 257-272, 1975.

[74] Johannsen, G. and T. Govinduraj, " Optimal Control Model Predictions of Systems Performance and Attention Allocation and Their Experimental Validation in a Display Design Study.", IEEE Trans. System, Man Cybernetics, vol. 10, pp. 249-261, 1984.

[75] Baron, S. and W. H. Levison, " An Optimal Control Methodology for Analyzing the Effects of Display Parameters on Performance and Work Load in Manual Flight Control.", IEEE Trans. System, Man, Cybernetics, vol. 5, pp. 423-430, 1975.

[76] Hess, R. A., " Analytical Display Design For Flight Tasks Controlled Under Instrument Meterological Conditions.", IEEE Trans. System, Man, Sybernetics, vol. 7, pp. 453-462, 1977

[77] Baron, S. and W. H. Levison, " Display Analusis With The Optimal Control Model of The Human Operator.", Human Factors, vol 19, pp. 437-457, 1977.

[78] Curry, R. E., D. 1. Kleinman and W. C. Hoffman.", A Design For Control/Display Systems.", Human Factors, vol. 19, pp. 421-436, 1977

[79] Hess, R. A., "Prediction of Pilot Ratins Using an Optimal Pilot Model.", Human Factors, vol. 19, pp. 459-475, 1977.

[80] Bryson, A. E., and Ho, Y. C., Applied optimal control, optimization, estimation, and control, John Wiley \& sons, NY, 1975.

[81] Isaacs, R., Differential Games, John Wiley \& sons, NY., 1965. 
[82] Ho, Y.C., A. E. Bryson, and Baron, S. , " Differential Games and optimal control pursuit-evasion strategies.," IEEE Trans. on Auto. Cont., vol. 10, 1965, pp. 385-389.

[83] Shapley, L. S., " Some Topics in Two-Person Game," In Annuals of math., Study N0. 52, Princeton, N. J., Princeton University Press., 1964, PP. 1-28.

[84] Berkowitz and Flemming, W. H., " On Differential Games with Integral Payoff," In Annuals of math., Study N0. 39, Princeton, N. J., Princeton University Press., 1957, PP. 413-445.

[85] Fleming, W. H., " The Convergence Problem for Differential Games," Journal of Mathematical Analysis \& Applications, vol. 3, 1961, pp. 102-116.

[86] Berkovitz, L. D., " A Variational Approach to Differential Games,", (Advances in Game Theory), in Annualy of Math. Study no. 52, Princeton, N. J., Princeton University Press., 1964, PP. 127-173.

[87]

---.-, A Differential Game With no Pure Strategy Solution," (Advances in Game Theory), in Annualy of Math. Study no. 52, Princeton, N. J., Princeton University Press., 1964, PP. 175-194.

[88] Wang, G. and Leitman, G., " Necessary and Sufficient Conditions for Multistags Two-Person, Zero-sum Games," Journal of Optimization Theory and Applications, vol. 4, no. 3, 1969, pp. 184-206.

[89] Chattopadhyay, R., " Completion of Linear Differential Games," Journal of Optimization Theory and Applications, vol. 3, no. 4, 1969, pp. 220-226.

[90] Lewis Meier, III, " A New Technique for Solving PursuitEvasion Differential Games," IEEE Trans on Auto. Control., vol. 14,1969 , pp. 352-359. 
[91] Salmon, D., "Polices and Controller Design for a Pursuing Vehicle,", IEEE Trans on Auto. Control, vol. 14, 1969, pp. $482-388$.

[92] Otomar Hajek, Pursuit Games: An Introduction to the theory and Applications of Differential Games of Pursuit and Evasion, Academic Press, N.Y., 1975.

[93] Porter, W., A., " On Function Space Pursuit-Evasion Games,", SIAM J. Control, vol. 5, no. 4, 1967, pp. 555-574.

[94] Moritz, K., Polis, R. and Well, K., " Pursuit-Evasion in MediumRange Air-Combat Scenarios,", Comput. Math. Applic. vol. 3, n0. 1-3, 1987, pp. 167-180.

[95] Mycielski, J., " Theories of Pursuit and Evasion,", Journal of Optimization Theory and Applications, vol. 56, no. 2, 1988, pp. 271-284.

[96] Pachter, M. and Kreindler, E., " An Optimal Evasion Problem: Complete Global Solution,", Journal of Optimization Theory and Applications, vol. 56, no. 3, 1988, pp. 179-204.

[97] Ho, Y. C., " Optimal Terminal Maneuver and Evasion Strategy,", J. SIAM Control, vol. 4, no. 3, 1966, pp. 421-429.

[98] Speyer, J. L., " A Stochastic Differential Game with Controllable Statistical Parameters," IEEE Trans. on Systems Science and Cybernetics, vol. 3, n. 1, 1967, pp. 17-20.

[99] Johnson, D., E., " Solution of a Linear Mean Square Estimation Problem Whwn Process Statistics are Undefined,", IEEE Trans. on Control, vol. 11, 1966, pp. 20-30.

[100] Luh, J. Y. S. and Maguiraga, M., " Differential Games with StateDependent and Control-Dependent Noise," IEEE Trans. on Auto. Control., vol. 16, no. 2, 1970, pp. 205-209.

[101] Behn, R. D. and Ho, Y., " On a Class of Linear Stochastic Differential Games,", IEEE Trans. on Auto. Control, vol. 13, 1968, pp. 227-240. 
[102] Rhodes, I. B. and Luenberger, D. G., " Differential Games with Imperfect State Information," IEEE Trans. on Auto. Control, vol.14, 1969, pp. 29-38.

[103] Willman, W., " Formal Solutions for a Class of Stochastic Pursuit-Evasion Games,", IEEE Trans. on Auto. Control, vol. 14, 1969, pp. 504-509.

[104] Rhodes, L. B., and Luenberger, D. G., " Stochastic Differential Games with Constrained State Estimators," IEEE Trans. on Auto. Control, vol. 14, 1969, pp. 476-481.

[105] Leondes, C. T. and Mons, B., " Differential Games with NoiseCorrupted Measurements," Journal of Optimization theory and Applications, vol. 28, no. 2, 1979, pp. 233-251.

[106] Leondes, C. T. and Mons, B., " On Line Solution of a Stochastic Pursuit-Evasion Game," Journal of Optimization theory and Applications, vol. 28, no. 3, 1979, pp. 411-429.

[107] Chan, W. L. and Ng, S. K., " Partially Observable LinearQuadratic Stochastic Pursuit-Evasion Games,", Comput. Math. Applic. Vol. 13, no. 1-3, pp. 181-189, 1987.

[108] Pontryagin, L. S., " On Some Differential Games," J. SIAM Control, vol. 3, pp. 49-52, 1965.

[109] Roxin, E., Liu, P. and Sternberg, R., Differential Games and Control Theory, Marcel Dekker, Inc., NY, 1977, pp. 49.

[110] Charles A. Baird, JR., " Modified Quasilinearization Technique for the Solution of Boundary-Value Problems for Ordinary Differential Equations," Journal of Optimization and Applications, vol. 3, no. 4, 1969.

[111] Miele, A. and Tyer, R. R., " General Technique for Solving Nonlinear, Two-Point Boundary-Value Problems Via the Method of Particular Solutions," Journal of Optimization and Applications, vol. 5, no. 3, 1970. 
[112] Bailey, P. B, Shampine, L. F, and Waltman, P. E., Nonlinear Two Point Boundary Value Problem, Academic Press, NY, 1968.

[113] Blaquiere, A., F. Gerard and Leitmann, G., Quantitative and Qualitative Games, Mathematics in Science and Engineering, vol. 58, 1969.

[114] Jaermark, B., " A Missle Dual Between two Aircraft,", Journal of Guid. and Control, vol. 8, pp. 508-513, 1985.

[115] Herbst, W., " Dynamics of air Combat," Journal of Aircraft, vol. 20, pp. 594-598, 1983.

[116] Basar, T. and Oldser, G. J., " Dynamic Noncooperative Game Theory," Mathematic in Science and Engineering, vol. 160, 1982.

[117] Starr, A. W. and Ho, Y., C., " Nonzero-sum Differential Games,", Journal of Optimization Theory and Applications, vol. 3, no. 3, 1969, pp. 184,219.

[118] Oldser, G. L. and Brekwell, J. V., " Role Determination in an Aerial Dogfigt," International Journal of Game Theory, vol. 3, pp. 47-66, 1974.

[119] Getz, W. M. and Leitmann, G., " Qualitative Differential Games with two Targets," Journal of Mathematics and Applications, vol. 68, pp. 421-430, 1979.

[120] Getz, W. M. and Pachter, M., " Two-Target Pursuit-Evasion Differential Games in the Plane," Journal of Optimization Theory and Applications, vol. 34, no. 3, pp. 382-402, 1981

[121] Stonier, R. J., " On Qualitative Differential Games with Two Targets," Journal of Optimization Theory and Applications, vol. 41, no. 4, 1983, pp. 587-599. 
[122] Yavin, Y., " Stochastic Two-Target Pursuit-Evasion Differential Games in the Plane,", Journal of Optimization theory and Applications, vol. 56, no. 3, 1988, pp. 325-343.

[123] Yavin, Y. and Villiers, R. DE, " Stochastic Pursuit-Evasion Differential Games in 3D," Journal of Optimization theory and Applications, vol. 56, no. 3, 1988, pp. 345-357. 


\section{APPENDIX A}

\section{OCM SOLUTION $[49,70]$}

\section{A.1 Introduction}

This appendix presents a detailed solution of the OCM (optimal controller for the systems with time delay and observation and motor noise) which was outlined in Chapter IV. Here, we investigate the problem of controlling a linear system to minimize a quadratic index of performance for the cases in which the output of the system is a delayed linear combination of system states corrupted by additive white observation noise.

In chapter III, we have seen that the optimal controller for linear stochastic system is produced by a linear feedback link consisting of optimal controller gains and a Kalman filter. This appendix will show how to modify this optimal controller for acceptance of the human's inherent constraints. In other words, we will see that the optimal control is generated by a linear feedback loop consisting of a cascaded combination of a Kalman filter, a least mean squared predictor, and a set of optimal controller gains. The effects of the human operator's limitations (time-delay, observation noise), which reduce system performance, will be seen explicitly in the derived expressions for the optimal performance in time domain as well as in frequency domain. 
We consider the system which is described by the state/output equations

$$
\begin{aligned}
& \underline{\dot{x}}(t)=A \underline{x}(t)+\underline{b} u(t)+E \underline{w}(t) \\
& \underline{y}(t)=C \underline{x}(t-\tau)+\underline{v}(t-\tau)
\end{aligned}
$$

where $\underline{w}(t)$ and $\underline{v}(t)$ are independent white Gaussian noises with intensity matrices

$$
\begin{aligned}
& E\left[\underline{w}(t) \underline{w}^{\prime}(\tau)\right]=W \delta(t-\tau) \\
& E\left[\underline{v}(t) \underline{v}^{\prime}(\tau)\right]=V \delta(t-\tau)
\end{aligned}
$$

The problem is to determine the control input $u(t)$ which minimizes the following performance index

$$
J=E\left[\underline{x}^{\prime} Q \underline{x}+r^{2}\right]
$$

where $Q$ is a positive semidefinite matrix and $\mathbf{r}$ is a positive scalar. Note that the case in which $\mathbf{J}$ contains a control rate term is considered later in this appendix.

\section{A.2 Optimal Controller}

\section{A.2.1 Solution of the Time Delay Problem}

First, the following case known as a prediction-control problem is solved.

Problem: For the system defined in (A-1) with the output

$$
\underline{y}(t)=\underline{x}(t-\tau),
$$


find the control input $\mathbf{u}^{*}(t)$ which minimizes the index of performance defined in (A-4).

This problem says that at time $t$ we are generating $\underline{x}(t-\tau)$ but not $\underline{x}(t)$ (see Equation (A-5)). In order to solve this problem, we first need to predict the current state, $\underline{x}(t)$, by observing the measurements $y(\delta)$, $\delta \leq \mathrm{t}$. The mean square prediction process has the form

$$
\underline{\hat{x}}(t)=E\{\underline{x}(t) \mid \underline{y}(\delta), \quad \delta \leq t\}
$$

In order to determine $\underline{\hat{x}}(t)$, we continue as follows. By differentiating both sides of (A-5) and using (A-1), we will have

$$
\begin{aligned}
\underline{\dot{y}}(t) & =\underline{\dot{x}}(t-\tau)=A \underline{x}(t-\tau)+\underline{b} u(t-\tau)+E_{\underline{w}}(t-\tau) \\
& =A \underline{y}(t)+\underline{b} u(t-\tau)+E_{\underline{w}}(t-\tau) .
\end{aligned}
$$

Knowing that the system (A-7) is linear, we may write

$$
\underline{y}(t)=\underline{y}_{u}(t)+\underline{y}_{w}(t)
$$

where $\underline{y}_{u}(t)$ and $\underline{y}_{w}(t)$ are respectively the contribution of $\underline{y}(t)$ due to $u(t)$ and due to $\underline{w}(t)$. They satisfy the following differential equations

$$
\begin{aligned}
& \dot{\dot{y}}_{u}(t)=A \underline{y}_{u}(t)+\underline{b} u(t-\tau) \\
& \underline{\dot{y}}_{w}(t)=A \underline{y}_{w}(t)+E \underline{w}(t-\tau) .
\end{aligned}
$$

Because of deterministic input $u(t)$ and randomness of $\underline{w}(t), \underline{y}_{u}(t)$ is a deterministic process and $\underline{\mathrm{y}}_{\mathrm{w}}(\mathrm{t})$ is a random process.

Now, if we write $\underline{x}(t)=\underline{y}(t+\tau)=\underline{y}_{u}(t+\tau)+\underline{y}_{w}(t+\tau)$, then we can find (using (A-6)) 


$$
\begin{aligned}
\underline{\hat{x}}(t) & =E\{\underline{x}(t) \mid \underline{y}(\sigma), \sigma \leq t\}= \\
& =E\left\{\underline{y}_{u}(t+\tau) \mid \underline{y}(\sigma), \sigma \leq t\right\}+E\left\{\underline{y}_{w}(t+\tau) \mid \underline{y}(\sigma), \sigma \leq t\right\} .
\end{aligned}
$$

Since $\underline{y}_{\mathfrak{a}}(t)$ is deterministic and it is independent of $\underline{y}_{w}(t)$, we write

$$
\underline{\hat{x}}(t)=\underline{y}_{u}(t+\tau)+E\left\{\underline{y}_{w}(t+\tau) \mid \underline{y}_{w}(\sigma), \sigma \leq t\right\}
$$

From (A-10), we may have

$$
\underline{\hat{x}}(t)=\underline{y}_{u}(t+\tau)+E\left\{\underline{y}_{w}(t+\tau) \mid \underline{y}_{w}(\sigma), \sigma \leq t\right\} .
$$

Knowing that $\underline{\mathrm{w}}($.$) is white noise and using the following properties of$ conditional expectation

$$
E[g(X) \mid X=x]=g(x)
$$

and

$$
\mathrm{E}[\mathrm{AX} \mid \mathrm{Y}=\mathrm{y}]=\mathrm{AE}[\mathrm{X} \mid \mathrm{Y}=\mathrm{y}],
$$

the second term in (A-12) becomes

$$
E\left\{\underline{y}_{w}(t+\tau) \mid \underline{y}_{w}(\sigma), \sigma \leq t\right\}=e^{A \tau} \underline{y}_{w}(t) .
$$

Thus,

$$
\underline{\hat{x}}(t)=\underline{y}_{u}(t+\tau)+e^{A \tau} \underline{y}_{w}(t)
$$

is the least mean squared prediction of $\underline{x}(t)$ with the following differential equation obtained from (A-14).

$$
\underline{\hat{\hat{x}}}(t)=\underline{\dot{y}}_{u}(t+\tau)+e^{A \tau} \underline{\dot{y}}_{w}(t)
$$

Using (A-9) and (A-10) in (A-15) yield 


$$
\underline{\dot{\hat{x}}}(\mathrm{t})=\mathrm{A} \underline{y}_{u}(t+\tau)+\underline{b} u(t)+e^{A \tau} A \underline{y}_{w}(t)+e^{A \tau} E \underline{w}(t-\tau) .
$$

Knowing that $\mathrm{e}^{\mathrm{A} \tau} \mathrm{A}=\mathrm{Ae} \mathrm{e}^{\mathrm{A} \tau}$, we can write

$$
\begin{aligned}
\underline{\dot{\hat{x}}}(t) & =A\left[\underline{y}_{u}(t+\tau)+e^{A \tau} \underline{y}_{w}(t)\right]+\underline{b}_{u}(t)+e^{A \tau} E_{\underline{w}}(t-\tau) \\
& =A \underline{\underline{\hat{x}}}(t)+\underline{b} u(t)+e^{A \tau} E_{\underline{w}}(t-\tau)
\end{aligned}
$$

which is of the same form as the system state equation given in (A-1). The prediction error is determined as

$$
\begin{aligned}
\underline{e}(t) & =\underline{x}(t)-\underline{\hat{x}}(t)=\underline{y}_{u}(t+\tau)+\underline{y}_{w}(t+\tau)-\underline{y}_{u}(t+\tau)-e^{A \tau} \underline{y}_{w}(t) \\
& =\underline{y}_{w}(t+\tau)-e \quad \underline{y}_{w}(t) .
\end{aligned}
$$

Using (A-13) in (A-18) gives

$$
\underline{e}(t)=\int_{t}^{t+\tau} e^{A(t+\tau-\sigma)} E \underline{w}(\sigma-\tau) d(\sigma) \text {. }
$$

In order to simplify (A-19), let $t+\tau-\sigma=\eta$. This implies

$$
\begin{aligned}
& d \sigma=-d \eta \\
& \sigma \in[t, t+\tau] \Rightarrow \eta \in[\tau, 0] .
\end{aligned}
$$

Thus, we can write (A-19) as

$$
\underline{e}(t)=-\int_{\tau}^{0} e^{A \eta} E_{\underline{w}}(t-\eta) d(\eta)=\int_{0}^{\tau} e^{A \eta} E_{\underline{w}}(t-\eta) d(\eta) .
$$

From (A-20), we can see that the control input $u(t)$ has no effect on the prediction error. Therefore, the minimum mean square 
error, which is calculated below, becomes independent of the choice of $u(t)$.

Covariance of $\underline{\mathrm{e}}(\mathrm{t})=\mathrm{E}\left\{\underline{\mathrm{e}}(\mathrm{t}) \underline{\mathrm{e}}^{\prime}(\mathrm{t})\right\}$

$$
\begin{aligned}
& =\int_{0}^{\tau} \int_{0}^{\tau} e^{A \eta} E\left\{E\left[\underline{w}(t-\eta) \underline{w^{\prime}}(t-\mu)\right] E^{\prime}\right\} e^{A^{\prime} \mu} d \eta d \mu \\
& =\int_{0}^{\tau} e^{A \eta} E W E^{\prime} d \eta \int_{0}^{\tau} \delta(\eta-\mu) e^{A^{\prime} \mu} d \mu \\
& =\int_{0}^{\tau} e^{A \eta} E W E^{\prime} e^{A^{\prime} \eta} d \eta .
\end{aligned}
$$

To obtain (A-21), we use the fact that $\left(e^{A \eta}\right)^{\prime}=e^{A^{\prime} \eta}$.

In order to minimize the index $\mathrm{J}(\mathrm{u})$, we first write

$$
\begin{aligned}
E\left\{\underline{x}^{\prime}(t) Q \underline{x}(t)\right\} & =E\left\{\underline{\hat{x}}^{\prime}(t) Q \underline{\hat{x}}(t)\right\}+E\left\{\underline{e}^{\prime}(t) Q \underline{e}(t)\right\} \\
& +E\left\{\underline{\hat{x}}^{\prime}(t) Q \underline{e}(t)\right\}+E\left\{\underline{e}^{\prime}(t) Q \underline{\hat{x}}(t)\right\} .
\end{aligned}
$$

Knowing that the predictor error is uncorrelated to the least mean square state estimate, i.e. $E\left\{\underline{\hat{x}}^{\prime}(t) \underline{e}(t)\right\}=E\left\{\underline{e}^{\prime}(t) \underline{\hat{x}}(t)\right\}=0$, the last two terms in (A-22) will be zero because of the following identity.

$$
\underline{w}^{\prime} \mathrm{A} \underline{\mathrm{w}}=\operatorname{Tr}\left[\mathrm{A} \underline{\mathrm{w}}^{\prime} \underline{\mathrm{w}}\right]=\operatorname{Tr}\left[\underline{\mathrm{w}}^{\prime} \underline{\mathrm{w}} \mathrm{A}\right]=\operatorname{Tr}\left[\underline{w}^{\prime} \underline{\mathrm{A}}^{\prime} \mathrm{A}\right] \text {. }
$$

Thus,

$$
E\left\{\underline{x}^{\prime}(t) Q \underline{x}(t)\right\}=E\left\{\underline{\hat{x}}^{\prime}(t) Q \underline{\hat{x}}(t)\right\}+E\left\{\underline{e}^{\prime}(t) Q \underline{e}(t)\right\} .
$$

Using (A-23) in (A-4) gives

$$
J(u)=E\left\{\underline{e}^{\prime}(t) Q \underline{e}(t)+\underline{\hat{x}}^{\prime}(t) Q \underline{\hat{x}}(t)+r u^{2}(t)\right\} .
$$

Substituting (A-21) and (A-23) into (A-25), we have 


$$
J(u)=\operatorname{Tr}\left(Q \int_{0}^{\tau} e^{A \eta} E W E^{\prime} e^{A^{\prime} \eta} d \eta\right)+J_{1}(u)
$$

where

$$
J_{1}(u)=E\left\{\underline{\hat{x}}^{\prime}(t) Q \underline{\hat{x}}(t)+r u^{2}(t)\right\}
$$

To minimize $J(u)$ we only need to minimize the index $J_{1}(u)$ defined in (A-27), because the first term in (A-26) is independent of the choice of $u$. In other words, the prediction-control problem changed to the problem of finding an input $\mathbf{u}^{*}(\mathrm{t})$ for the system given in (A-17) so that the index of performance $J_{1}(u)$ is minimized. This is a regulator problem which was discussed in Chapter III. Its solution is

$$
u^{*}(t)=-F \underline{\hat{x}}(t), F=r^{-1} \underline{b}^{\prime} P
$$

where $P$ is the unique positive definite solution of the Riccati equation

$$
\mathrm{Q}+\mathrm{PA}+\mathrm{A}^{\prime} \mathrm{P}-\mathrm{P} \underline{\mathrm{b}} \mathbf{r}^{-1} \underline{b}^{\prime} \mathrm{P}=0
$$

with the optimal cost

$$
\mathrm{J}_{1}^{*}\left(\mathrm{u}^{*}\right)=\operatorname{Tr}\left(\mathrm{PW}_{1}\right)
$$

where $W_{1}=e^{A \tau} E W E^{\prime} e^{A^{\prime} \tau}$ is the intensity matrix of the white process $\underline{w}_{1}(t)=e^{A \tau} \operatorname{Ew}(t-\tau)$.

In summary, as illustrated in Figure A-1, the solution of the prediction-control problem is given by equation (A-28), where $\underline{\hat{x}}(t)$ described by Equation (A-17) is the least mean square prediction of 


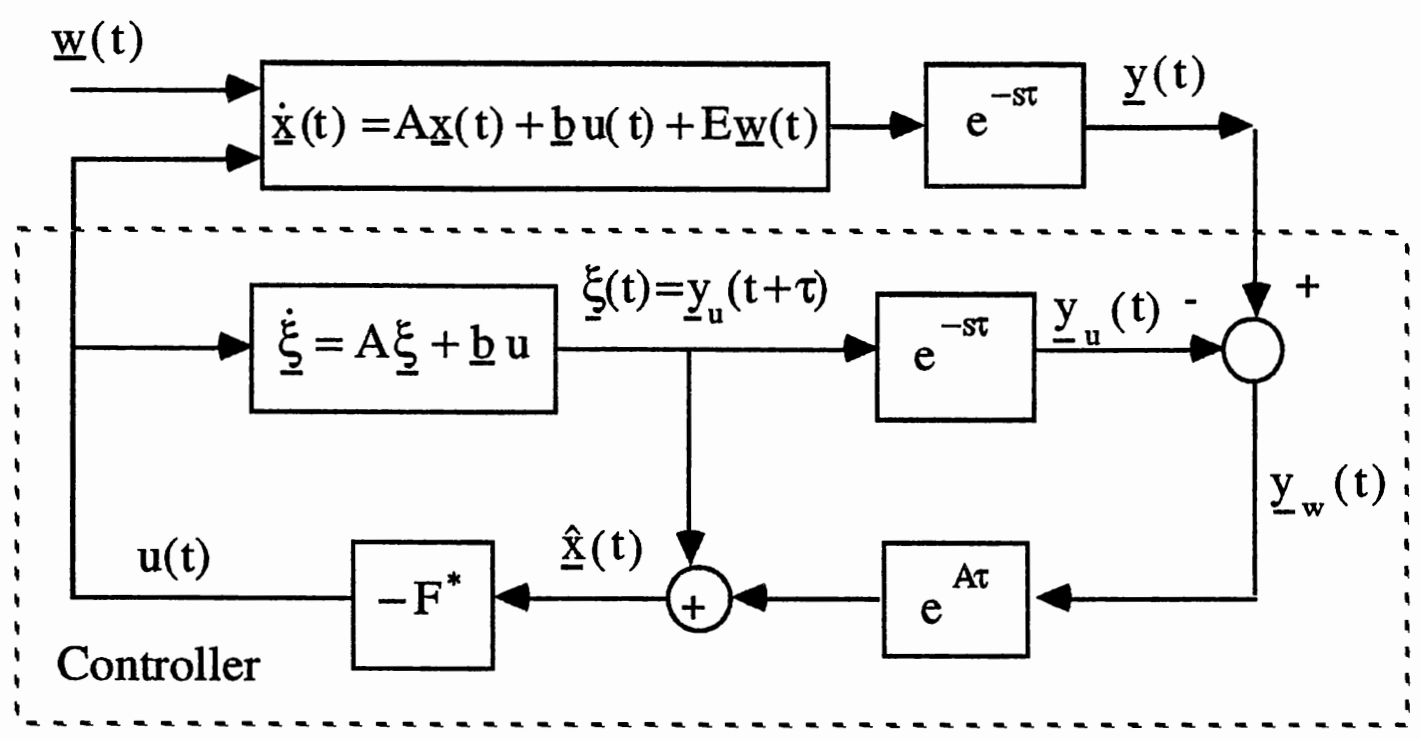

Figure A.1 Optimal Feedback Controller for System With Time Delay

$\underline{x}(t)$, with the optimal index of performance

$$
J\left(u^{*}\right)=\operatorname{Tr}\left(Q \int_{0}^{\tau} e^{A \eta} E W E^{\prime} e^{A^{\prime} \eta} d \eta\right)+\operatorname{Tr}\left(P^{A \tau} E W E^{\prime} e^{A^{\prime} \tau}\right)
$$

\section{A.2.2 Optimal Controller for Noisy \\ Time-Delay Systems.}

This section solves for the optimal control for the systems whose operations described by equations (A-1) and (A-2) introduced in section A.1. Let 


$$
\underline{\hat{x}}(\mathrm{t}-\tau)=E\{\underline{\mathrm{x}}(\mathrm{t}-\tau) \mid \underline{\mathrm{y}}(\sigma), \sigma \leq \mathrm{t}\}
$$

be the least mean squared estimate of $\underline{x}(t-\tau)$. As discussed in Chapter III, this estimate can be found by means of a Kalman filter as shown below,

$$
\underline{\dot{\hat{x}}}(\mathrm{t}-\tau)=\mathrm{A} \underline{\hat{x}}(\mathrm{t}-\tau)+\underline{\mathrm{b}} \mathrm{u}(\mathrm{t}-\tau)+\mathrm{K}[\underline{\mathrm{y}}(\mathrm{t})-\mathrm{C} \underline{\hat{x}}(\mathrm{t}-\tau)]
$$

where

$$
\begin{aligned}
& \underline{y}(t)=C \underline{x}(t-\tau)+\underline{v}(t-\tau) \\
& K=\Sigma C^{\prime} V^{-1}
\end{aligned}
$$

and $\Sigma$ is the covariance matrix of the reconstruction error

$$
\underline{e}(t)=\underline{x}(t)-\underline{\hat{x}}(t) \text {. }
$$

$\Sigma$ satisfies the matrix Riccati equation

$$
\mathrm{A} \Sigma+\Sigma \mathrm{A}^{\prime}+\mathrm{EWE}^{\prime}-\Sigma \mathrm{C}^{\prime} \mathrm{V}^{-1} \mathrm{C} \Sigma=0
$$

Using (A-1) and (A-33) in (A-35), we can have the following equation which defines the error $\underline{e}(t)$.

$$
\underline{\dot{e}}(t)=[A-K C] \underline{e}(t)-K \underline{v}(t)+E \underline{w}(t)
$$

From Equation (A-37) we see that $\underline{e}(t)$ is independent of $u(t)$. By substituting (A-33) in (A-4) and proceeding as in section A.2.1 (see equation (A-25), the performance index $J(u)$ becomes

$$
J(u)=E\left\{\underline{e}^{\prime}(t-\tau) Q \underline{e}(t-\tau)+\underline{\hat{x}}^{\prime}(t-\tau) Q \underline{\hat{x}}(t-\tau)+r u^{2}(t-\tau)\right\} .
$$


Knowing that

$$
\mathrm{E}\left\{\underline{\mathrm{e}}^{\prime}(\mathrm{t}-\tau) \mathrm{Q} \underline{\mathrm{e}}(\mathrm{t}-\tau)\right\}=\operatorname{Tr}\left(\mathrm{QE}\left\{\underline{\mathrm{e}}^{\prime}(\mathrm{t}-\tau) \underline{\mathrm{e}}(\mathrm{t}-\tau)\right\}\right)=\operatorname{Tr}(\mathrm{Q} \Sigma),
$$

$\mathbf{J}(\mathbf{u})$ is written as

$$
\mathrm{J}(\mathrm{u})=\mathrm{J}_{1}(\mathrm{u})+\operatorname{Tr}(\mathrm{Q} \Sigma)
$$

where

$$
J_{1}(u)=E\left\{\underline{\hat{x}}^{\prime}(t-\tau) Q \underline{\hat{x}}(t-\tau)+r u^{2}(t-\tau)\right\} .
$$

Therefore, minimizing of $J(u)$ is equivalent to minimizing of $J_{1}(u)$.

By letting $\underline{p}(t)=\left.\underline{\hat{x}}(t-\tau)\right|_{t=t+\tau}$, Equation $(A-40)$ and (A-33) can be rewritten as

$$
\begin{aligned}
& J_{1}(u)=E\left\{\underline{p}(t) Q \underline{p}(t)+r u^{2}(t)\right\} \\
& \underline{\dot{p}}(t)=A \underline{p}(t)+\underline{b} u(t)+\underline{\tilde{w}}(t)
\end{aligned}
$$

where

$$
\tilde{\mathbf{w}}(\mathrm{t})=\mathrm{K}[\mathrm{C} \underline{\mathrm{e}}(\mathrm{t})+\underline{\mathrm{v}}(\mathrm{t})] \text {. }
$$

It has been shown that $\tilde{w}(t)$ is a white noise process [49] with the intensity matrix

$$
E\{\underline{\tilde{w}}(t) \underline{\tilde{w}}(\sigma)\}=\tilde{W} \delta(t-\sigma)
$$

where

$$
\tilde{\mathrm{W}}=\mathrm{KC} \Sigma=\Sigma \mathrm{C}^{\prime} \mathrm{V}^{-1} \mathrm{C} \Sigma
$$


From Equation (A-42) we can see that at time $t$ we really produce $\underline{\hat{x}}(t-\tau)$ not $\underline{\hat{x}}(t)$. This implies that the control input $u(t)$ can only be generated by $\underline{\hat{x}}(\sigma), \sigma \leq t-\tau$. Now, we have the problem of minimizing $J_{1}(u)$ defined in Equation (A-41) subject to (A-42). This is exactly the prediction-control problem which was discussed in the previous section. Therefore, the control input $u^{*}(t)$ which minimizes $\mathrm{J}_{1}(\mathrm{u})$ is

$$
u^{*}(t)=-F \underline{\gamma}(t)
$$

where $\underline{\gamma}(t)$ is the output of the least mean squared predictor with the input $\underline{p}(t) . \underline{\gamma}(t)$ satisfies the following equations

$$
\begin{aligned}
& \underline{\gamma}(\mathrm{t})=\underline{\xi}(\mathrm{t})+\mathrm{e}^{\mathrm{A} \tau}[\underline{\mathrm{p}}(\mathrm{t})-\underline{\xi}(\mathrm{t}-\tau)] \\
& \underline{\xi}(\mathrm{t})=\mathrm{A} \underline{\xi}(\mathrm{t})+\underline{\mathrm{b}} \mathrm{u}^{*}(\mathrm{t}) .
\end{aligned}
$$

Figure A-2 shows the closed optimal control system for this case. As indicated in this Figure, the optimal feedback link is a cascaded combination a Kalman filter, a linear mean squared predictor, and optimal controller gain factors. 


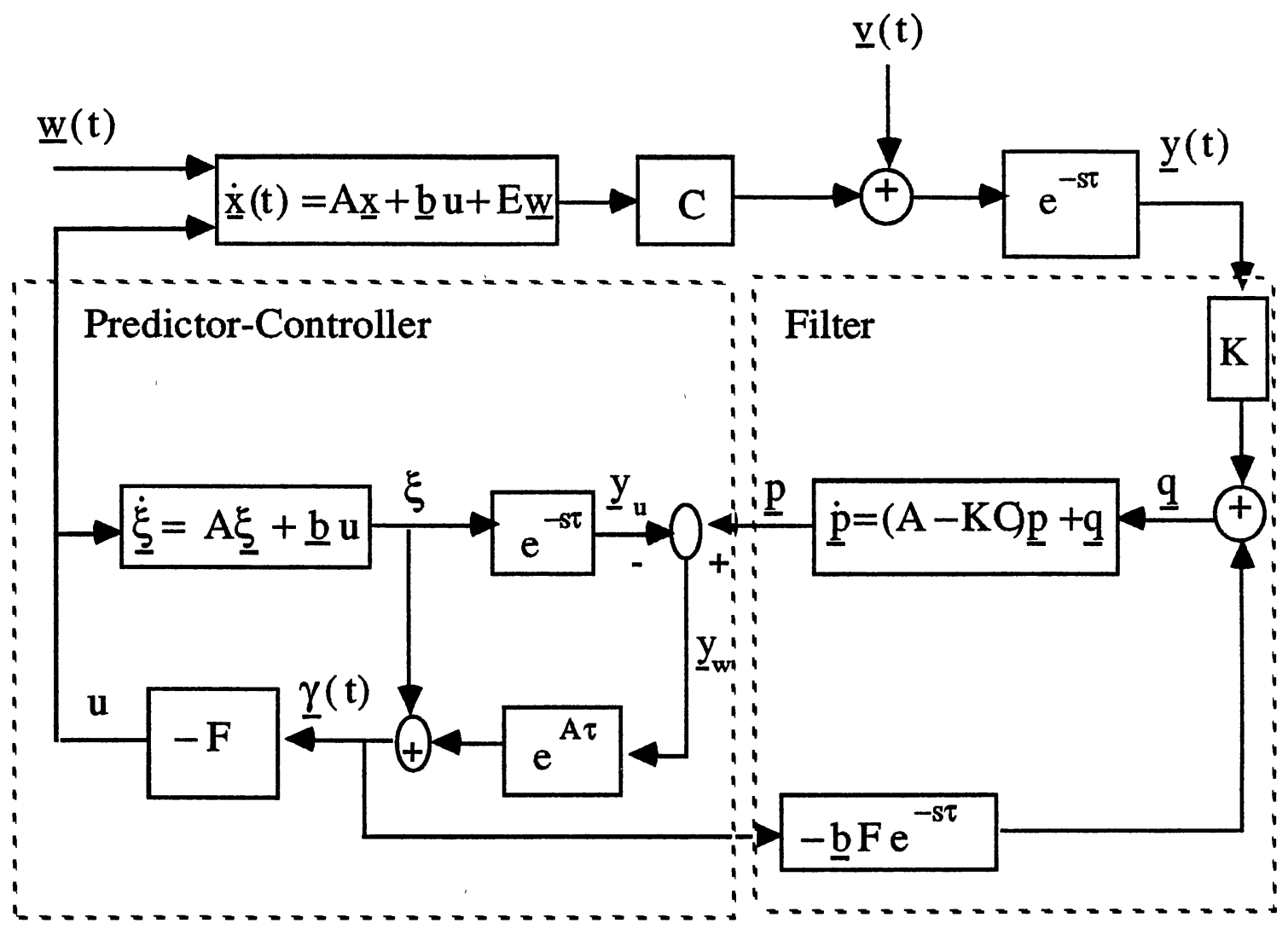

Figure A-2. Optimal Controller for Systems with Time-Delay and Observation Noise

\section{A.2.3 The Evaluation of Performance Index}

In order to evaluate the index $J(u)$, we first need to find the minimum value of $J_{1}(u)$ from Equation (A-40). Using (A-44) in (A-31), the optimum of $J_{1}(u)$ is

$$
\begin{aligned}
\mathrm{J}\left(\mathrm{u}_{1}^{*}\right) & =\operatorname{Tr}\left(\mathrm{Q} \int_{0}^{\tau} \mathrm{e}^{\mathrm{A} \eta} \tilde{\mathrm{W}} \mathrm{e}^{\mathrm{A}^{\prime} \eta} \mathrm{d} \eta\right)+\operatorname{Tr}\left(\mathrm{Pe}^{\mathrm{A} \tau} \tilde{\mathrm{W}} \mathrm{e}^{\mathrm{A}^{\prime} \tau}\right) \\
& =\operatorname{Tr}\left(\mathrm{Q} \int_{0}^{\tau} \mathrm{e}^{\mathrm{A} \eta} \Sigma \mathrm{C}^{\prime} \mathrm{V}^{-1} \mathrm{C} \Sigma \mathrm{e}^{\mathrm{A}^{\prime} \eta} \mathrm{d} \eta\right)+\operatorname{Tr}\left(\mathrm{Pe}^{\mathrm{A} \tau} \Sigma \mathrm{C}^{\prime} \mathrm{V}^{-1} \mathrm{C} \Sigma \mathrm{e}^{\mathrm{A}^{\prime} \tau}\right) .
\end{aligned}
$$


From (A-36), we can write

$$
\Sigma \mathrm{C}^{\prime} \mathrm{V}^{-1} \mathrm{C} \Sigma=\mathrm{A} \Sigma+\Sigma \mathrm{A}^{\prime}+\mathrm{EWE}^{\prime}
$$

By substituting (A-49) into (A-48), Equation (A-39) becomes

$$
\begin{aligned}
\mathrm{J}\left(\mathrm{u}^{*}\right)= & \mathrm{J}_{1}\left(\mathrm{u}^{*}\right)+\operatorname{Tr}(\mathrm{Q} \Sigma) \\
= & \operatorname{Tr}\left(\mathrm{Q} \int_{0}^{\tau} \mathrm{e}^{\mathrm{A} \eta} \mathrm{EWE} \mathrm{e}^{\mathrm{A}^{\prime} \eta} \mathrm{d} \eta\right) \\
& +\operatorname{Tr}\left(\mathrm{Q}\left[\mathrm{A} \int_{0}^{\tau} \mathrm{e}^{\mathrm{A} \eta} \Sigma \mathrm{e}^{\mathrm{A}^{\prime} \eta} \mathrm{d} \eta+\int_{0}^{\tau} \mathrm{e}^{\mathrm{A} \eta} \Sigma \mathrm{e}^{\mathrm{A}^{\prime} \eta} \mathrm{d} \eta \mathrm{A}^{\prime}+\Sigma\right]\right) \\
& +\operatorname{Tr}\left(\mathrm{Pe}^{\mathrm{A} \tau} \mathrm{EWE} \mathrm{e}^{\mathrm{A}^{\prime} \tau}\right)+\operatorname{Tr}\left(\left[\mathrm{PA}+\mathrm{A}^{\prime} \mathrm{P}\right] \mathrm{e}^{\mathrm{A} \tau} \Sigma \mathrm{e}^{\mathrm{A}^{\prime} \tau}\right) .
\end{aligned}
$$

It can be shown that (using power series expansion)

$$
\mathrm{A} \int_{0}^{\tau} \mathrm{e}^{\mathrm{A} \eta} \Sigma \mathrm{e}^{\mathrm{A}^{\prime} \eta} \mathrm{d} \eta+\int_{0}^{\tau} \mathrm{e}^{\mathrm{A} \eta} \Sigma \mathrm{e}^{\mathrm{A}^{\prime} \eta} \mathrm{d} \eta \mathrm{A}^{\prime}+\Sigma=\mathrm{e}^{\mathrm{A} \tau} \Sigma \mathrm{e}^{\mathrm{A}^{\prime} \tau}
$$

Using (A-51) in (A-50), the optimal performance index $J\left(u^{*}\right)$ becomes

$$
\begin{aligned}
\mathrm{J}\left(\mathrm{u}^{*}\right) & =\operatorname{Tr}\left(\mathrm{Q} \int_{0}^{\tau} \mathrm{e}^{\mathrm{A} \eta} \mathrm{EWE} \mathrm{e}^{\mathrm{A}^{\prime} \eta} \mathrm{d} \eta\right)+\operatorname{Tr}\left(\mathrm{Pe}^{\mathrm{A} \tau} \mathrm{EWE} \mathrm{e}^{\mathrm{A}^{\prime} \tau}\right) \\
& +\operatorname{Tr}\left(\left[\mathrm{PA}+\mathrm{A}^{\prime} \mathrm{P}+\mathrm{Q}\right] \mathrm{e}^{\mathrm{A} \tau} \Sigma \mathrm{e}^{\mathrm{A}^{\prime} \tau}\right) .
\end{aligned}
$$

Using (A-29) in (A-52), $J\left(\mathbf{u}^{*}\right)$ will be

$$
\begin{aligned}
\mathrm{J}\left(\mathrm{u}^{*}\right) & =\operatorname{Tr}\left(\mathrm{Q} \int_{0}^{\tau} \mathrm{e}^{\mathrm{A} \eta} \mathrm{EWE} \mathrm{e}^{\mathrm{A}^{\prime} \eta} \mathrm{d} \eta\right)+\operatorname{Tr}\left(\mathrm{Pe}^{\mathrm{A} \tau} \mathrm{EWE} \mathrm{e}^{\mathrm{A}^{\prime} \tau}\right) \\
\cdot & +\operatorname{Tr}\left(\mathrm{P}_{\mathrm{b}} \mathbf{r}^{-1} \underline{\mathrm{b}} \mathrm{Pe}^{\mathrm{A} \tau} \Sigma \mathrm{e}^{\mathrm{A}^{\prime} \tau}\right) .
\end{aligned}
$$


By letting $\mathrm{P} \underline{\mathrm{b}}=\underline{v}, \mathrm{Pe}^{\mathrm{A} \tau} \Sigma \mathrm{e}^{\mathrm{A}^{\prime} \tau}=\mathrm{Z}$, and using the trace property $\underline{v} \mathbf{Z} \underline{v}=\operatorname{Tr}\left(\underline{v}^{\prime} \underline{Z} \mathrm{Z}\right)$ given in $(\mathrm{A}-23)$, the index $(\mathrm{A}-53)$ can be rewritten as:

$$
\begin{aligned}
J\left(u^{*}\right) & =\operatorname{Tr}\left(Q \int_{0}^{\tau} e^{A \eta} E W E^{\prime} e^{A^{\prime} \eta} d \eta\right)+ \\
& +\operatorname{Tr}\left(\operatorname{Pe}^{A \tau} E^{A} E^{\prime} e^{A^{\prime} \tau}\right)+F_{e} \Sigma F_{e}^{\prime}
\end{aligned}
$$

where

$$
\mathrm{F}_{\mathrm{e}}=\sqrt{\mathrm{r}} \mathrm{r}^{-1} \underline{\mathrm{b}}^{\prime} \mathrm{Pe} \mathrm{e}^{\mathrm{A} \tau}=\sqrt{\mathrm{r}} \mathrm{Fe} \mathrm{A}^{\mathrm{A} \tau}
$$

The effects of human operator's limitations (time-delay and observation noise) are explicitly shown in the optimal performance index given by Equation (A-55).

In order to evaluate the closed-loop covariance matrix of system state and control input, we may continue as follows. Using (A-28) in (A-17) and (A-27), the positive definite matrix $P$ satisfies the Lyapunov equation [63]

$$
\mathrm{PA}_{\mathrm{c}}+\mathrm{A}_{\mathrm{c}}^{\prime} \mathrm{P}+\mathrm{Q}_{\mathrm{c}}=0
$$

with the solution

$$
P=\int_{0}^{\infty} e^{A_{c} \sigma} Q_{c} e^{A_{c}^{\prime} \sigma} d \sigma=\int_{0}^{\infty} e^{A_{c}^{\prime} \sigma} Q_{c} e^{A_{c} \sigma} d \sigma
$$

where

$$
A_{c}=A-\underline{b} F
$$

and 


$$
Q_{c}=Q+F^{\prime} r F .
$$

After introducing (A-49), (A-52) can be written

$$
\begin{aligned}
\mathrm{J}\left(\mathrm{u}^{*}\right) & =\operatorname{Tr}\left(\mathrm{Q} \int_{0}^{\tau} \mathrm{e}^{\mathrm{A} \eta} E W E^{\prime} \mathrm{e}^{\mathrm{A}^{\prime} \eta} \mathrm{d} \eta\right)+\operatorname{Tr}\left(\mathrm{Pe}^{\mathrm{A} \tau} \mathrm{EWE} \mathrm{e}^{\mathrm{A}^{\prime} \tau}\right) \\
& +\operatorname{Tr}\left(\mathrm{Q} \mathrm{e}^{\mathrm{A} \tau} \Sigma \mathrm{e}^{\mathrm{A}^{\prime} \tau}\right)+\operatorname{Tr}\left(\mathrm{Pe}^{\mathrm{A} \tau} \mathrm{A} \Sigma \mathrm{e}^{\mathrm{A}^{\prime} \tau}\right)+\operatorname{Tr}\left(\mathrm{Pe}^{\mathrm{A} \tau} \Sigma \mathrm{A}^{\prime} \mathrm{e}^{\mathrm{A}^{\prime} \tau}\right) \\
& =\operatorname{Tr}\left(\mathrm{Q} \int_{0}^{\tau} \mathrm{e}^{\mathrm{A} \eta} \mathrm{EWE} \mathrm{e}^{\mathrm{A}^{\prime} \eta} \mathrm{d} \eta\right)+\operatorname{Tr}\left(\mathrm{Q} \mathrm{e}^{\mathrm{A} \tau} \Sigma \mathrm{e}^{\mathrm{A}^{\prime} \tau}\right) \\
& +\operatorname{Tr}\left(\mathrm{Pe}^{\mathrm{A} \tau} \Sigma \mathrm{C}^{\prime} \mathrm{V}^{-1} \mathrm{C} \Sigma \mathrm{e}^{\mathrm{A}^{\prime} \tau}\right) .
\end{aligned}
$$

Substituting (A-56) into (A-58) gives

$$
\begin{aligned}
\mathrm{J}\left(\mathbf{u}^{*}\right) & =\operatorname{Tr}\left(\mathrm{Q} \int_{0}^{\tau} \mathrm{e}^{\mathrm{A} \eta} E W E^{\prime} \mathrm{e}^{\mathrm{A}^{\prime} \eta} \mathrm{d} \eta\right)+\operatorname{Tr}\left(\mathrm{Q} \mathrm{e}^{\mathrm{A} \tau} \Sigma \mathrm{e}^{\mathrm{A}^{\prime} \tau}\right) \\
& +\operatorname{Tr}\left(\int_{0}^{\infty} \mathrm{e}^{\mathrm{A}_{\mathrm{c}}^{\prime} \sigma} \mathrm{Q}_{\mathrm{c}} \mathrm{e}^{\mathrm{A}_{\mathrm{c}} \sigma} \mathrm{e}^{\mathrm{A} \tau} \Sigma \mathrm{C}^{\prime} \mathrm{V}^{-1} \mathrm{C} \Sigma \mathrm{e}^{\mathrm{A}^{\prime} \tau} \mathrm{d} \sigma\right) \\
\mathrm{J}\left(\mathbf{u}^{*}\right) & =\operatorname{Tr}\left(\mathrm{Q} \int_{0}^{\tau} \mathrm{e}^{\mathrm{A} \eta} E W E^{\prime} \mathrm{e}^{\mathrm{A}^{\prime} \eta} \mathrm{d} \eta\right)+\operatorname{Tr}\left(\mathrm{Q} \mathrm{e}^{\mathrm{A} \tau} \Sigma \mathrm{e}^{\mathrm{A}^{\prime} \tau}\right) \\
& +\operatorname{Tr}\left(\mathrm{Q}_{\mathrm{c}} \int_{0}^{\infty} \mathrm{e}^{\mathrm{A}_{\mathrm{c}}^{\prime} \sigma} \mathrm{e}^{\mathrm{A} \tau} \Sigma \mathrm{C}^{\prime} \mathrm{V}^{-1} \mathrm{C} \Sigma \mathrm{e}^{\mathrm{A}^{\prime} \tau} \mathrm{e}^{\mathrm{A}_{\mathrm{c}} \sigma} \mathrm{d} \sigma\right) .
\end{aligned}
$$

Using (A-57), (A-59) becomes

$$
\begin{aligned}
& J\left(u^{*}\right)=\operatorname{Tr}\left(Q_{0}\left\{\begin{array}{l}
\int_{0}^{\tau} e^{A \eta} E W E^{\prime} e^{A^{\prime} \eta} d \eta+e^{A \tau} \Sigma e^{A^{\prime} \tau}+ \\
\int_{0}^{\infty} e^{A_{c}^{\prime} \sigma} e^{A \tau} \Sigma C^{\prime} V^{-1} C \Sigma e^{A^{\prime} \tau} e^{A_{c} \sigma} d \sigma
\end{array}\right\}+\right. \\
& +\operatorname{Tr}\left(F^{\prime} r F \int_{0}^{\infty} e^{A_{c}^{\prime} \sigma} e^{A \tau} \Sigma C^{\prime} V^{-1} C \Sigma e^{A^{\prime} \tau} e^{A_{c} \sigma} d \sigma\right) \text {. }
\end{aligned}
$$


By equating the right hand sides of equations (A-60) and (A-4), we will have the following identities

$$
\begin{aligned}
& \mathrm{E}\left[\underline{x}^{\prime} \mathrm{Q} \underline{\mathrm{x}}\right]=\operatorname{Tr}\left(\mathrm{QE}\left[\underline{x}^{\prime} \underline{x}\right]\right)= \\
& \left.\operatorname{Tr}\left(\begin{array}{l}
\left.\int_{0}^{\tau} \mathrm{e}^{\mathrm{A} \eta} \mathrm{EWE} \mathrm{e}^{\mathrm{A}^{\prime} \eta} \mathrm{d} \eta+\mathrm{e}^{\mathrm{A} \tau} \Sigma \mathrm{e}^{\mathrm{A}^{\prime} \tau}+\right) \\
\int_{0}^{\infty} \mathrm{e}^{\mathrm{A}_{\mathrm{c}}^{\prime} \sigma} \mathrm{e}^{\mathrm{A} \tau} \Sigma \mathrm{C}^{\prime} \mathrm{V}^{-1} \mathrm{C} \Sigma \mathrm{e}^{\mathrm{A}^{\prime} \tau} \mathrm{e}^{\mathrm{A}_{\mathrm{c}} \sigma} \mathrm{d} \sigma
\end{array}\right\}\right) \\
& E\left[\mathrm{ru}^{2}\right]=\operatorname{Tr}\left(\mathrm{F}^{\prime} \mathrm{rF} \int_{0}^{\infty} \mathrm{e}^{\mathrm{A}_{\mathrm{c}}^{\prime} \sigma} \mathrm{e}^{\mathrm{A} \tau} \Sigma \mathrm{C}^{\prime} \mathrm{V}^{-1} \mathrm{C} \Sigma \mathrm{e}^{\mathrm{A}^{\prime} \tau} \mathrm{e}^{\mathrm{A}_{\mathrm{c}} \sigma} \mathrm{d} \sigma\right) .
\end{aligned}
$$

From (A-61), the covariance matrix of the closed loop state is

$$
\begin{aligned}
\operatorname{Cov}(\underline{x})=E\left[\underline{x}^{\prime} \underline{x}\right]= & \int_{0}^{\tau} e^{A \eta} E W E^{\prime} e^{A^{\prime} \eta} d \eta+e^{A \tau} \Sigma e^{A^{\prime} \tau}+ \\
& \int_{0}^{\infty} e^{A_{c}^{\prime} \sigma} e^{A \tau} \Sigma C^{\prime} V^{-1} C \Sigma e^{A^{\prime} \tau} e^{A_{c} \sigma} d \sigma .
\end{aligned}
$$

Another approach for determination of $\operatorname{Cov}(\underline{x})$ can be conducted as follows. Knowing that the least mean squared estimator is uncorrelated with its associated error, we can write the covariance of the closed-loop system state as

$$
\begin{aligned}
\operatorname{Cov}(\underline{\mathrm{x}}) & =\operatorname{Cov}(\text { filter error })+\operatorname{Cov}(\operatorname{Pr} \text { edictor Error }) \\
& +\operatorname{Cov}(\operatorname{Pr} \text { edictor })= \\
& =\Sigma+\int_{0}^{\tau} \mathrm{e}^{\mathrm{A} \eta} \Sigma \mathrm{C}^{\prime} \mathrm{V}^{-1} \mathrm{C} \Sigma \mathrm{e}^{\mathrm{A}^{\prime} \eta} \mathrm{d} \eta+\operatorname{Cov}(\underline{\gamma}) .
\end{aligned}
$$

The last term in (A-64) can be found as follows. By referring to Figure (A-2) and introducing (A-10), we can write the following equations.

$$
\underline{\gamma}(t)=\underline{\xi}(t)+e^{A \tau} \underline{y}_{w}(t)
$$




$$
\begin{aligned}
\underline{\dot{\gamma}}(\mathrm{t}) & =\mathrm{A} \underline{\xi}(\mathrm{t})+\mathrm{e}^{\mathrm{A} \tau}\left[\mathrm{A} \underline{y}_{\mathrm{w}}(\mathrm{t})+\underline{\tilde{w}}(\mathrm{t}-\tau)\right]-\underline{\mathrm{b}} \mathrm{F} \underline{\gamma}(\mathrm{t}) \\
& =\mathrm{A} \underline{\gamma}(\mathrm{t})-\mathrm{Ae} \mathrm{e}^{\mathrm{A} \tau} \underline{\mathrm{y}}_{\mathrm{w}}(\mathrm{t})+\mathrm{e}^{\mathrm{A} \tau} \mathrm{A} \underline{y}_{\mathrm{w}}(\mathrm{t})+\mathrm{e}^{\mathrm{A} \tau} \underline{\tilde{\mathrm{w}}}(\mathrm{t}-\tau)-\underline{\mathrm{b}} \mathrm{F} \underline{\gamma}(\mathrm{t}) \\
& =\mathrm{A}_{\mathrm{c}} \underline{\gamma}(\mathrm{t})+\mathrm{e}^{\mathrm{A} \tau} \underline{\tilde{\mathrm{w}}}(\mathrm{t}-\tau) .
\end{aligned}
$$

with $A_{c}$ defined in (A-57). For the above differential equation, the covariance of $\underline{\gamma}(t)$ is [63]

$$
\operatorname{Cov}(\underline{\gamma})=\int_{0}^{\infty} \mathrm{e}^{\mathrm{A}_{c}^{\prime} \sigma} \mathrm{e}^{\mathrm{A} \tau} \tilde{\mathrm{W}} \mathrm{e}^{\mathrm{A}^{\prime} \tau} \mathrm{e}^{\mathrm{A}_{\mathrm{c}} \sigma} \mathrm{d} \sigma
$$

where

$$
\tilde{\mathrm{W}}=\operatorname{Cov}(\underline{\tilde{\mathrm{w}}})=\Sigma \mathrm{C}^{\prime} \mathrm{V}^{-1} \mathrm{C} \Sigma \text {. }
$$

Using (A-65) in (A-64), we will obtain equation (A-63), since from Equations (A-49) and (A-51), it has been shown that

$$
\Sigma+\int_{0}^{\tau} e^{A \eta} \Sigma C^{\prime} V^{-1} C \Sigma e^{A^{\prime} \eta} d \eta=\int_{0}^{\tau} e^{A \eta} E W E^{\prime} e^{A^{\prime} \eta} d \eta+e^{A \tau} \Sigma e^{A^{\prime} \tau} .
$$

To summarize, so far the problem of finding an optimal controller for linear systems with time delay and observation noise has been solved and it has been seen that the optimal feedback control is a cascaded combination of a Kalman filter, least mean squared predictor and optimal controller gain factors. The optimal performance index and the covariance of closed-loop system state have been determined in their closed forms which explicitly reflect the effects of human inherent limitations (time-delay and observation noise) on overall system performance.

As discussed in chapter IV, applying OCM in manual control systems requires us to consider a index of performance which contains 
a control rate term in order to restrict rapid control movements. This kind of control problem is discussed in next section.

\section{A.2.4 The OCM Solution}

In this section we consider the problem stated as: for the system defined by equations (A-1) \& (A-2), we are looking for a control input $u^{*}(t)$ which minimizes the following performance index

$$
J(u)=E\left\{\underline{x} Q \underline{x}+r u^{2}+g \dot{u}^{2}\right\} .
$$

In order to convert this optimal control problem to the one discussed in section (A.2.2), we make the following modifications.

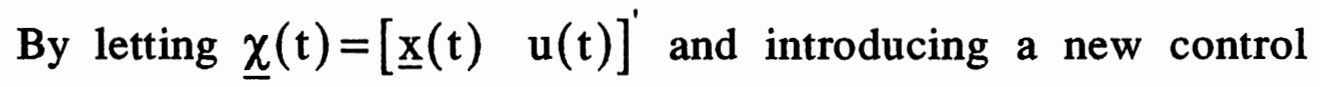
$\mu(t)=\dot{u}(t)$, we will have the $(n+1)$-dimensional system

$$
\begin{aligned}
& \underline{\dot{\chi}}(t)=A_{1} \underline{\chi}(t)+\underline{b}_{1} \mu(t)+E_{1} \underline{w}(t) \\
& \underline{y}(t)=C_{1} \underline{\chi}(t-\tau)+\underline{v}(t-\tau)
\end{aligned}
$$

with the index

$$
\mathrm{J}(\mathrm{u})=\mathrm{E}\left\{\underline{\underline{\chi}} \mathrm{Q}_{1} \underline{\chi}+\mathrm{g} \mu^{2}\right\}
$$

where

$$
\begin{aligned}
& \mathrm{A}_{1}=\left[\begin{array}{cc}
\mathrm{A} & \underline{\mathrm{b}} \\
0_{1 \times \mathrm{n}} & 0
\end{array}\right], \underline{\mathrm{b}}_{1}=\left[\begin{array}{c}
0_{\mathrm{n} \times 1} \\
1
\end{array}\right], \mathrm{C}_{1}=\left[\begin{array}{ll}
\mathrm{C} & \mathrm{d}
\end{array}\right], \\
& \mathrm{Q}_{1}=\left[\begin{array}{cc}
\mathrm{Q} & 0_{\mathrm{n} \times 1} \\
0_{1 \times \mathrm{n}} & \mathrm{r}
\end{array}\right], \mathrm{E}_{1}=\left[\begin{array}{l}
\mathrm{E} \\
0_{1 \times \mathrm{n}_{\mathrm{w}}}
\end{array}\right] .
\end{aligned}
$$


Now, we want to determine the control input $\mu *(t)$ which minimizes (A-69) subjected to (A-67) \& (A-68). This is exactly the same type of problem discussed in section (A.2.2). In order to apply the results obtained in the previous sections, $A_{1}, \underline{b}_{1}, C_{1}, Q_{1}, E_{1}$ must replace $A, b, C, Q, E$, respectively. The optimal control input is

$$
\mu^{*}(\mathrm{t})=-\mathrm{F} \underline{\hat{\chi}}(\mathrm{t})
$$

where

$$
\begin{aligned}
& F=g^{-1} \underline{b}_{1}^{\prime} P=\left[\begin{array}{lllll}
f_{1} & . & f_{n} & f_{n+1}
\end{array}\right] \\
& P A_{1}+A_{1}^{\prime}+Q_{1}-g^{-1} P \underline{b}_{1} \underline{b}_{1}^{\prime} P=0(n+1) \times(n+1)
\end{aligned}
$$

The error covariance matrix is determined by Equation (A-36) with appropriate matrix replacements mentioned above.

$$
\mathrm{A}_{1} \Sigma_{1}+\Sigma_{1} \mathrm{~A}_{1}^{\prime}+\mathrm{E}_{1} \mathrm{WE}_{1}^{\prime}-\Sigma_{1} \mathrm{C}_{1}^{\prime} \mathrm{V}^{-1} \mathrm{C}_{1} \Sigma_{1}=0_{(\mathrm{n}+1) \times(\mathrm{n}+1)}
$$

Using (A-70) in (A-73) and solving for $\Sigma_{1}$, we have

$$
\Sigma_{1}=\left[\begin{array}{cc}
\Sigma & 0_{\mathrm{n} \times 1} \\
0_{1 \times n} & 0
\end{array}\right]
$$

where $\Sigma$ satisfies the equation (A-36). This implies

$$
\hat{\chi}_{n+1}=u, \hat{\chi}_{i}=\hat{x}_{i}, \quad i=1,2, \ldots, n
$$

(A-74) says that the consideration of control rate as a new control input has no effect on state estimator, $\underline{\hat{x}}(t)$ and the Kalman filter generates an exact estimate of control input $u(t)$. The latter is 
unrealistic from a human operator viewpoint, since he has no perfect knowledge of the controlled system input. Thus, as indicated in Figure 4.1, the motor noise which is white Gaussian process with intensity $V_{u}$, is added to the commanded input signal. The effect of the motor noise will be discussed later.

The human's time constant lag $\tau_{\mathrm{n}}$ and optimal controller gain $\mathrm{F}^{*}$ can be determined as follows. By using (A-72) in (A-71), we can write

$$
\mu^{*}=\dot{\mathrm{u}}=-\mathrm{f}_{\mathrm{n}+1} \mathrm{u}-\left[\mathrm{f}_{1} \cdot \mathrm{f}_{\mathrm{n}}\right] \underline{\hat{\mathrm{x}}} .
$$

By taking the Laplace transform of both sides of (A-75), we will have

$$
\mathrm{U}(\mathrm{s})=\frac{1}{\tau_{\mathrm{n}} \mathrm{s}+1}\left(-\mathrm{F}^{*} \underline{\hat{\mathrm{X}}}(\mathrm{s})\right)
$$

where

$$
\tau_{n}=\frac{1}{f_{n+1}}, F^{*}=\tau_{n}\left[\begin{array}{llll}
f_{1} & . & f_{n}
\end{array}\right] .
$$

As seen, both $F^{*}$ and $\tau_{n}$ are only dependent on the system parameters $A$ and $\underline{b}$ and cost weightings introduced in the performance index. For fixed values of $Q$ and $r$ there exists a 1-to-1 correspondence between $g$ and $\tau_{\mathrm{n}}$. As $\mathrm{g}$ becomes smaller the input magnitude becomes larger which in turn requests for the larger gain factors (e.g., $f_{n+1}$ ). This implies smaller $\tau_{n}$. Hence, the smaller $g$ yields the smaller $\tau_{n}$.

Equation (A-76) shows that the substitution of the control rate term in the performance index is equivalent to the introduction of a first order lag with time constant $\tau_{\mathbf{n}}$ into the optimal controller (as indicated in Figure (4.1)). 
In order to see the effect of the motor noise $v_{u}(t)$, which represents the imperfect knowledge of the control input imposed by human operator, we assume that the control input $u(t)$ is generated by

$$
\begin{aligned}
& \dot{u}(t)=-\frac{1}{\tau_{n}} u(t)+\frac{1}{\tau_{n}} u_{c}(t)+\frac{1}{\tau_{n}} v_{u}(t) \\
& u_{c}(t)=-F^{*} \underline{\hat{x}}(t)
\end{aligned}
$$

where $\mathrm{F}^{*}$ and $\tau_{\mathrm{n}}$ are defined in (A-77). By combining Equations (A-1) and (A-78), we can write

$$
\underline{\dot{\chi}}(\mathrm{t})=\mathrm{A}_{2} \underline{\chi}(\mathrm{t})+\underline{\mathrm{b}}_{2} \mathrm{u}_{\mathrm{c}}(\mathrm{t})+\mathrm{E}_{2} \underline{\mathrm{w}}_{1}(\mathrm{t})
$$

where

$$
\mathrm{A}_{2}=\left[\begin{array}{cc}
\mathrm{A} & \underline{\mathrm{b}} \\
0_{1 \times n} & -\frac{1}{\tau_{\mathrm{n}}}
\end{array}\right], \underline{\mathrm{b}}_{2}=\left[\begin{array}{c}
0_{\mathrm{n} \times 1} \\
\frac{1}{\tau_{\mathrm{n}}}
\end{array}\right], \underline{\mathrm{w}}_{1}(\mathrm{t})=\left[\begin{array}{c}
\underline{w}(\mathrm{t}) \\
\mathrm{v}_{\mathrm{u}}(\mathrm{t})
\end{array}\right], \mathrm{E}_{2}=\left[\begin{array}{cc}
\mathrm{E} & 0_{\mathrm{n} \times 1} \\
0_{1 \times \mathrm{n}_{\mathrm{w}}} & \frac{1}{\tau_{\mathrm{n}}}
\end{array}\right]
$$

with

$$
E\left\{\underline{w}_{1}^{\prime}(t) \underline{w}_{1}(\sigma)\right\}=\left[\begin{array}{cc}
W & 0_{n \times 1} \\
0_{1 \times n} & \frac{V_{u}}{\tau_{n}^{2}}
\end{array}\right] \delta(t-\sigma)=W_{1} \delta(t-\sigma) .
$$

For the system given by (A-79) and (A-68), we wish to find the control input minimizing the index of performance defined in (A-69). This problem has been solved in the previous sections. Using the previous results with appropriate matrix replacements, we can write the following equations for Kalman filter, least mean squared predictor, and the closed loop system state covariance matrix. 
The state estimate $\underline{\hat{x}}(t)$ is obtained by cascade combination of an optimal state estimator (Kalman filter) and a least mean squared predictor. The Kalman filter produces a least mean square delayed estimate, $\underline{\hat{x}}(t-\tau)$, of the delayed state $\underline{x}(t-\tau)$ b $y$

$$
\underline{\dot{\gamma}}(t)=A_{2} \underline{\gamma}(t)+\underline{b}_{2} u_{c}(t-\tau)+K\left[\underline{y}(t)-C_{2} \underline{\gamma}(t)\right]
$$

where

$$
\underline{\gamma}(\mathrm{t})=\left[\begin{array}{l}
\hat{\hat{x}}(\mathrm{t}-\tau) \\
\hat{\mathrm{u}}(\mathrm{t}-\tau)
\end{array}\right], \mathrm{C}_{2}=\left[\begin{array}{ll}
\mathrm{C} & \mathrm{d}
\end{array}\right] .
$$

The optimal estimator gain matrix, $\mathrm{K}$ is

$$
\mathrm{K}=\Gamma \mathrm{C}_{2}^{\prime} \mathrm{V}_{\mathrm{y}}^{-1}
$$

where $\Gamma$ is the error intensity matrix and the unique symmetric positive definite solution of the Riccati equation

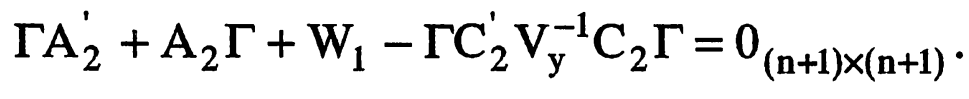

The predictor yields the optimum estimate $\underline{\hat{\chi}}(t)=\left[\begin{array}{l}\hat{\underline{x}}(t) \\ \hat{u}(t)\end{array}\right]$ from the Kalman filter output $\underline{\gamma}(t)$. The following equations define the linear dynamics of the predictor

$$
\begin{aligned}
& \underline{\hat{\chi}}(\mathrm{t})=\underline{\xi}(\mathrm{t})+\mathrm{e}^{\mathrm{A}_{2} \tau}[\underline{\gamma}(\mathrm{t})-\underline{\xi}(\mathrm{t}-\tau)] \\
& \underline{\dot{\xi}}(\mathrm{t})=\mathrm{A}_{2} \underline{\xi}(\mathrm{t})+\underline{\mathrm{b}}_{2} \mathrm{u}_{\mathrm{c}}^{*}(\mathrm{t})
\end{aligned}
$$

and the optimal commanded input is

$$
u_{c}^{*}(t)=-\left[\begin{array}{ll}
F^{*} & 0
\end{array}\right] \underline{\hat{\chi}}(t)
$$


The following expression is a closed-loop form for the covariance matrix of $\underline{\chi}(t)$.

$$
\begin{aligned}
X=E\left\{\underline{\chi}(t) \underline{\chi}(t)^{\prime}\right\}= & e^{A_{2} \tau} \Gamma e^{A_{2}^{\prime} \tau}+\int_{0}^{\tau} e^{A_{2} \sigma} E_{2} W_{1} E_{2}^{\prime} e^{A_{2}^{\prime} \sigma} d \sigma+ \\
& \int_{0}^{\infty} e^{\bar{A}_{2} \sigma} e^{A_{2} \tau} \Gamma C_{2}^{\prime} V_{y}^{-1} C_{2} \Gamma e^{A_{2}^{\prime} \tau} e^{\bar{A}_{2} \sigma} d \sigma
\end{aligned}
$$

where

$$
\overline{\mathrm{A}}=\mathrm{A}_{2}-\underline{\mathrm{b}}_{2}\left[\begin{array}{ll}
\mathrm{F}^{*} & 0
\end{array}\right]
$$

Since $\underline{\chi}()=.\left[\begin{array}{l}\underline{x}(.) \\ u(.)\end{array}\right]$, the variance of the system quantities are:

$$
\begin{aligned}
& \mathrm{E}\left[\mathrm{x}_{\mathrm{i}}^{2}(\mathrm{t})\right]=\mathrm{x}_{\mathrm{ii}} \quad \text { For } \mathrm{i}=1, \ldots, \mathrm{n} \\
& E\left[y_{i}^{2}(t)\right]=\left\{\left[\begin{array}{ll}
C & d
\end{array}\right] x\left[\begin{array}{l}
C^{\prime} \\
\underline{d}^{\prime}
\end{array}\right]\right\}_{i i} \quad \text { For } i=1, \ldots, m \\
& E\left[u^{2}(t)\right]=X_{(n+1),(n+1)} \text {. }
\end{aligned}
$$

The effects of human limitations on the overall closed-loop manmachine system are explicitly reflected in the equations (A-86) \& (A87).

Being linear and time-invariant, the structure of the OCM can be formulated in the frequency domain by a linear transfer function linking $y$ to $u$ in the form 


$$
\begin{aligned}
U(s)=\underline{h}(s) \underline{Y}(s) & =\left[\begin{array}{llll}
h_{1}(s) & \cdot & h_{m}(s)
\end{array}\right]\left[\begin{array}{c}
Y_{1}(s) \\
\cdot \\
\cdot \\
Y_{m}(s)
\end{array}\right] \\
& =\sum_{1}^{m} h_{i}(s) Y_{i}(s)
\end{aligned}
$$

where $T_{i}(s)$ denotes the Laplace transform of $t_{i}(s)$. The next section develops the vector transfer function $\underline{\mathbf{h}}(\mathrm{s})$.

\section{A.3, Human Operator's Transfer \\ Function Development}

The linear and time-invariant structure of optimal controller has been expressed in the time-domain by the Equations ( A-79)-(A-85). This section presents the frequency domain structure of feedback controller by a vector transfer function which relate the input, $y$, to the output, u, of the human operator, e. g.,

$$
u(s)=\underline{h}(s) \underline{y}(s) .
$$

Here, we derive the expression for $\underline{h}(s)$ from block-diagram manipulations as follows. Figure A-3 represents a frequency domain representation of the human operator. In order to simplify the blockdiagrams shown in Figure A-3, we use the block-diagram reduction rules given in table 1. By applying the reduction rules of table 1 to Figure A-3, we can determine the transfer function $\underline{h}(s)$ linking $y$ to $u$. Initially, we use Rule (a) to move the summing point (labeled with ${ }^{*}$ ) to 
the left of the block which contains $e^{A_{1} \tau}$. Next, we use Rule (b) to move the block labeled with \# to the right of the first summing point from left. The results of these block transformations are shown in Figure A4. Finally, we use Rule (c) to obtain the equivalent transfer function block in Figure A-4.c.

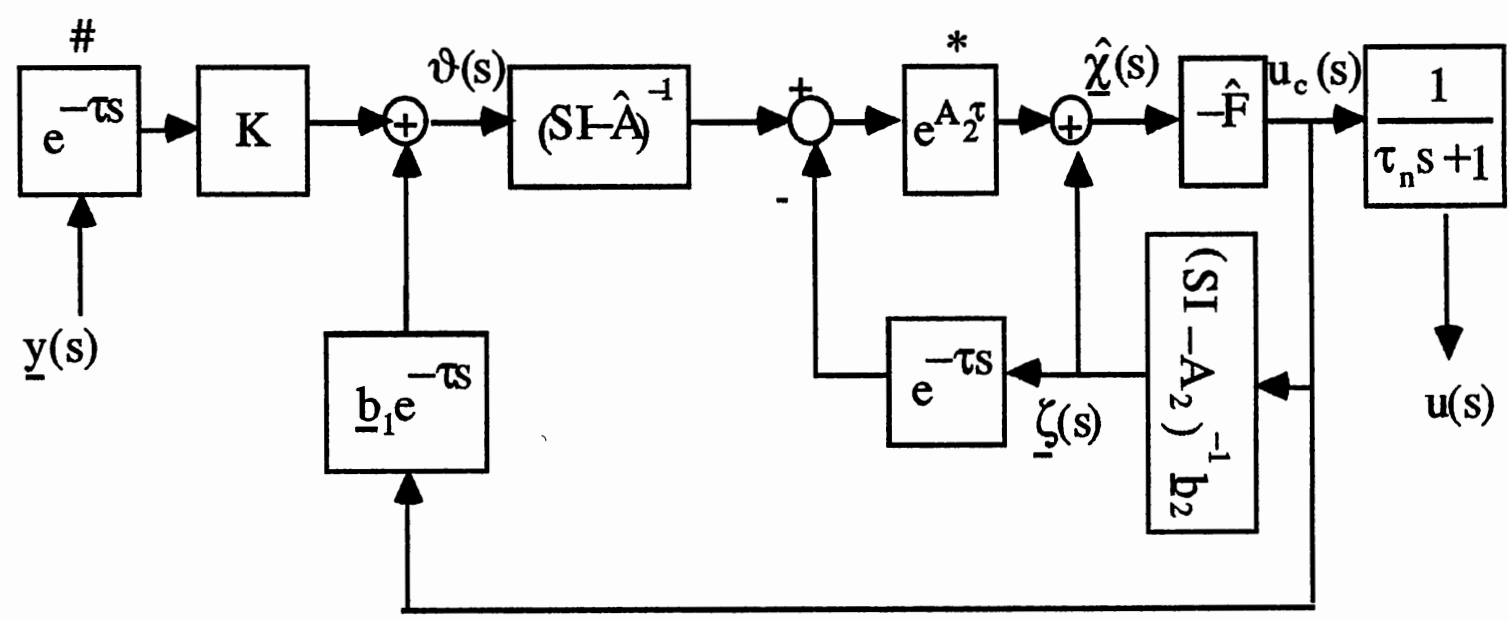

Figure A.3. Frequency Domain Representation of Human Operator 
Table A.1

Block-Diagram Matrix Reduction Rules

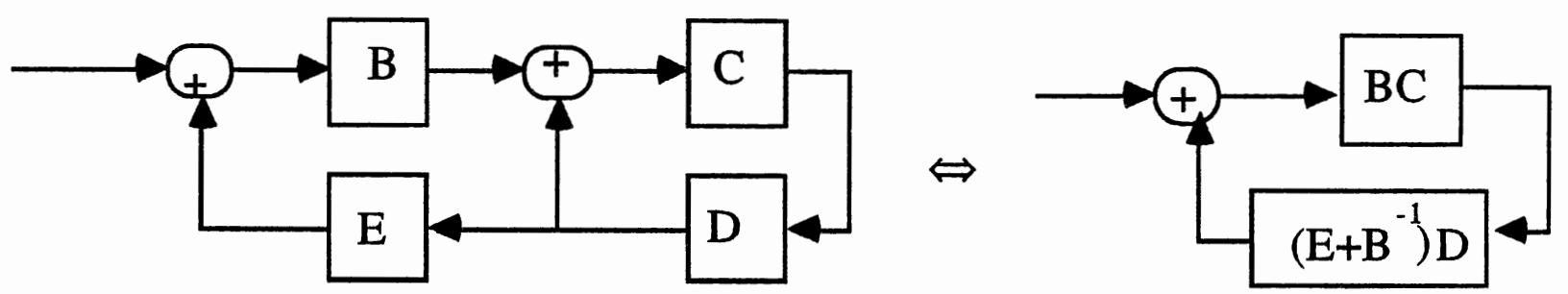

(a)
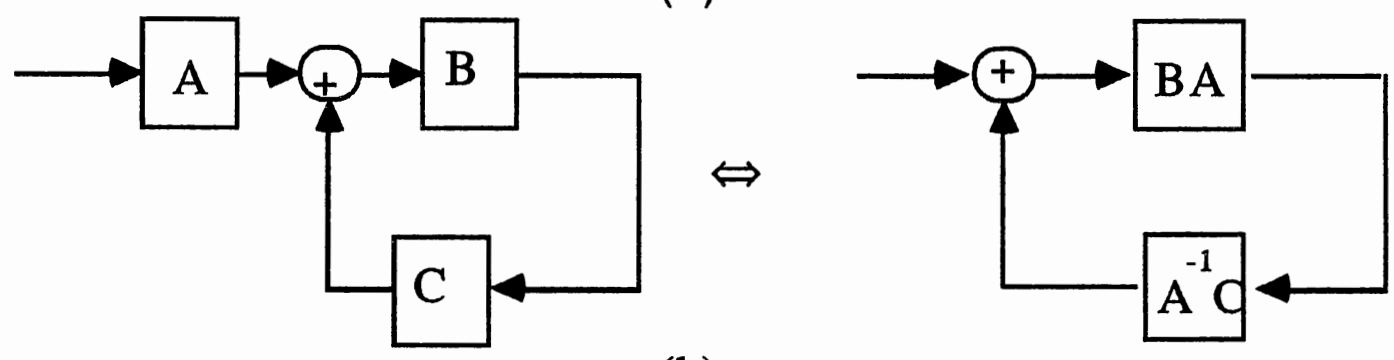

(b)

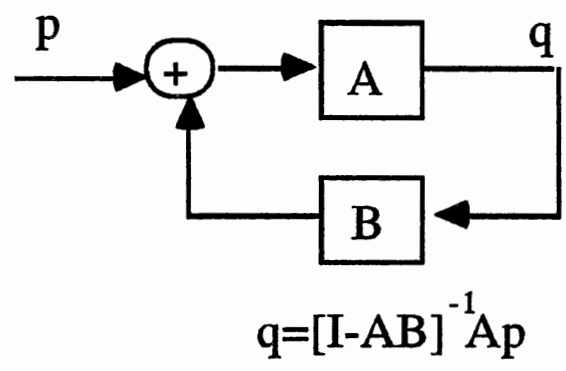




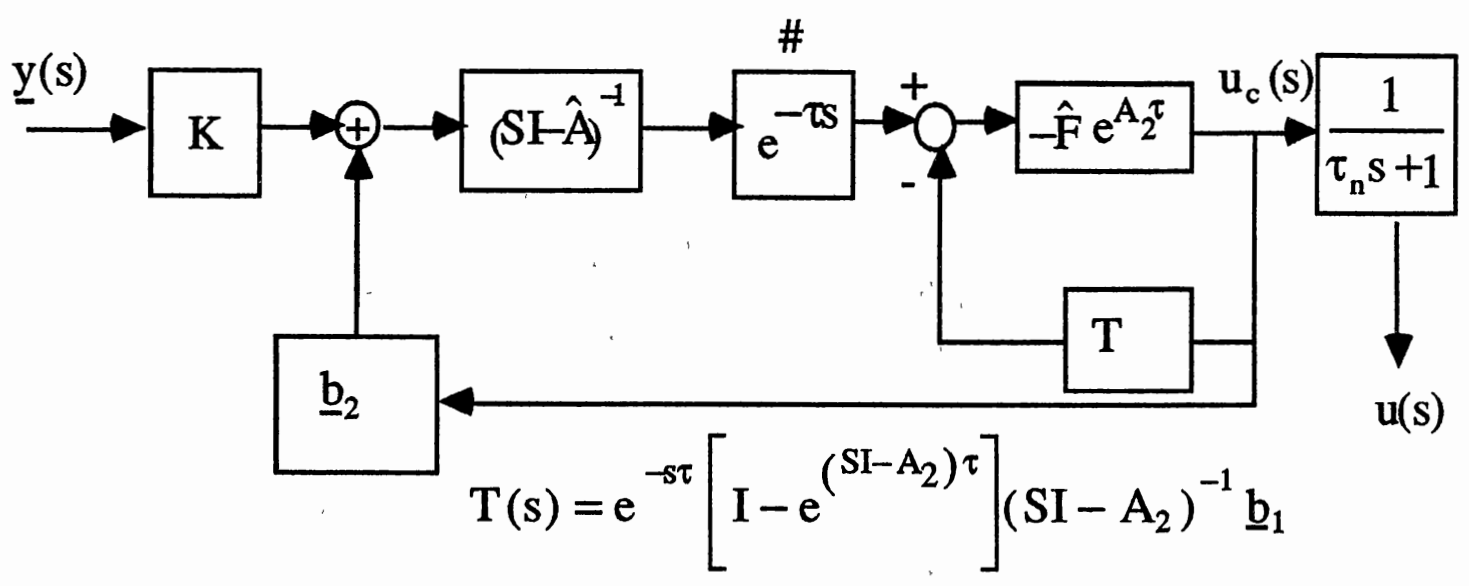

(a)

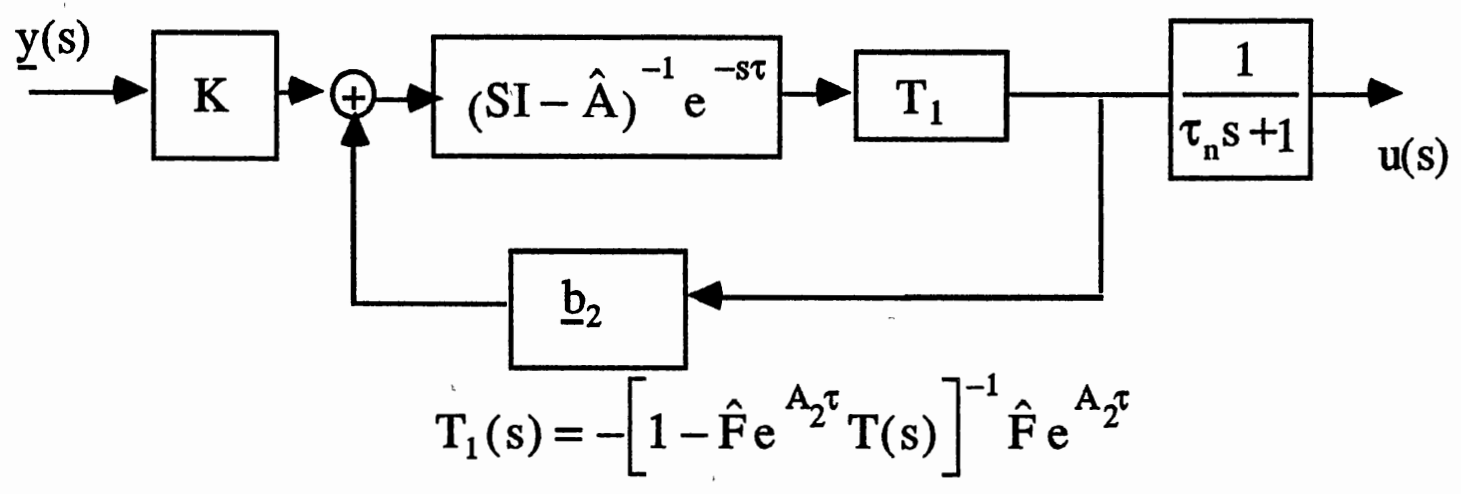

(b)

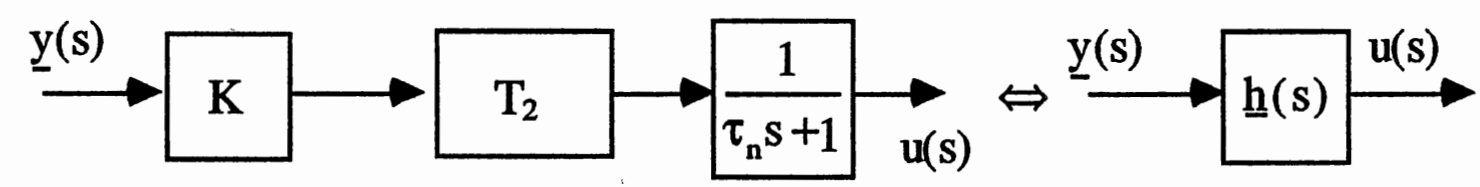

$T_{2}=\left[1-T_{1} e^{-s \tau}(S I-\hat{A})^{-1} \underline{b}_{2}\right]^{-1} T_{1} e^{-s \tau}(S I-\hat{A})^{-1} \Leftrightarrow \underline{h}(s)=\frac{1}{\tau_{n} s+1} T_{2} K$

(c)

Figure A-4. Application of Rules Given in Table A-1 to Figure A-3 
$T_{1}(s)$ and $T_{2}(s)$ can be simplified as:

$$
\begin{aligned}
\mathrm{T}_{1}(\mathrm{~s})= & -[1-\varphi(\mathrm{s})]^{-1} \hat{\mathrm{F}} \mathrm{e}^{-\mathrm{A}_{2} \tau} \\
\mathrm{T}_{2}(\mathrm{~s})= & -\left\{1+[1-\varphi(\mathrm{s})]^{-1} \hat{\mathrm{F}} \mathrm{e}^{\left(\mathrm{A}_{2}-\mathrm{SI}\right) \tau}(\mathrm{SI}-\hat{\mathrm{A}})^{-1} \underline{\mathrm{b}}_{2}\right\}^{-1} \times \\
& \left\{[1-\varphi(\mathrm{s})]^{-1} \hat{\mathrm{F}} \mathrm{e}^{\left(\mathrm{A}_{2}-\mathrm{SI}\right) \tau}(\mathrm{SI}-\hat{\mathrm{A}})^{-1}\right\}
\end{aligned}
$$

where

$$
\varphi(s)=\hat{F} e^{\left(A_{2}-S I\right) \tau}\left(I-e^{\left(s I-A_{2}\right) \tau}\right)\left(S I-A_{2}\right)^{-1} \underline{b}_{2}
$$

and

$$
\hat{\mathrm{F}}=\left[\begin{array}{ll}
\mathrm{F}^{*} & 0
\end{array}\right]
$$

Since $\varphi(s)$ is scalar, $T_{2}(s)$ can be written

$$
\mathrm{T}_{2}(\mathrm{~s})=-\left\{1-\varphi(\mathrm{s})+\hat{\mathrm{F}} \mathrm{e}^{\left(\mathrm{A}_{2}-\mathrm{SI}\right) \tau}(\mathrm{SI}-\hat{\mathrm{A}})^{-1} \underline{\mathrm{b}}_{2}\right\}^{-1} \hat{\mathrm{F}} \mathrm{e}^{\left(\mathrm{A}_{2}-\mathrm{SI}\right) \tau}(\mathrm{SI}-\hat{\mathrm{A}})^{-1}
$$

By Substituting (A-88) into (A-89), we will have

$$
\begin{aligned}
T_{2}(s)= & -\left\{1+\hat{F}\left[e^{\left(A_{2}-S I\right) \tau}\left((S I-\hat{A})^{-1}-\left(S I-A_{2}\right)^{-1}\right)+\left(S I-A_{2}\right)^{-1}\right] \underline{b}_{2}\right\}^{-1} \times \\
& \hat{F} e^{\left(A_{2}-S I\right) \tau}(S I-\hat{A})^{-1} .
\end{aligned}
$$


Finally, the vector transfer function $\underline{\mathrm{h}}(\mathrm{s})$ becomes

$$
\begin{aligned}
\underline{\mathrm{h}}(\mathrm{s})= & -\frac{1}{\tau_{\mathrm{n}} \mathrm{s}+1}\left\{\hat{\mathrm{F}} \mathrm{e}^{\left(\mathrm{A}_{2}-\mathrm{SI}\right) \tau}(\mathrm{SI}-\hat{\mathrm{A}})^{-1} \mathrm{~K}\right\} \times \\
& \left\{1+\hat{\mathrm{F}}\left[\mathrm{e}^{\left(\mathrm{A}_{2}-\mathrm{SI}\right) \tau}\left((\mathrm{SI}-\hat{\mathrm{A}})^{-1}-\left(\mathrm{SI}-\mathrm{A}_{2}\right)^{-1}\right)+\left(\mathrm{SI}-\mathrm{A}_{2}\right)^{-1}\right] \underline{\mathrm{b}}_{2}\right\}^{-1} .
\end{aligned}
$$

To summarize, the OCM consists of three key elements: Kalman filter, minimum mean squared predictor, and the optimal controller which assigns a set of control weights to the the elements of the predictor. For a given controlled system the OCM is completely determined by the quadratic cost weightings, the time delay $\tau$, and the intensities of the observation and motor noise; the effects of human limitations on the overall closed-loop man-machine system in time domain as well as in frequency domain are explicitly reflected in the equations (A-86) \& (A-90). 


\section{APPENDIX B}

\section{STABILIZABILITY OF THE SYSTEM}

Here, we shall show that the system defined by the state equation of the form

$$
\underline{\dot{x}}_{7}(t)=A_{8} \underline{x}_{7}(t)+\underline{b}_{8} u_{d_{2}}(t)
$$

where

$$
A_{8}=\left[\begin{array}{cc}
A_{7} & 0 n_{x_{6} \times n_{x_{6}}} \\
0 n_{\mathrm{n}_{2}} \times n_{x_{6}} & A_{2}
\end{array}\right], \underline{b}_{8}=\left[\begin{array}{c}
0 n_{x_{6} \times 1} \times 1 \\
\underline{b}_{2}
\end{array}\right],
$$

$A_{7}=\left[\begin{array}{cc}A_{6} & 0_{2 n_{x 4} \times n_{y 1} \cdot n_{x_{d} 3}} \\ B_{D_{3}} C_{5} & A_{D_{3}}^{\prime}\end{array}\right], A_{D_{3}}=\left[\begin{array}{llll}A_{d_{3}} & & & \\ & A_{d_{3}} & & \\ & & \cdot & \\ & & \cdot & \\ & & & A_{d_{3}}\end{array}\right]$,

is stabilizable. The matrices and parameters in (B-2) were defined in section 5.2.2.

To show the stabilizability of the system (B-1), the following theorem taken from [79] is used. 
Theorem. Consider the time-invariant linear system

$$
\underline{\dot{x}}(t)=\left[\begin{array}{cc}
0_{p \times q} & A_{12} \\
A_{21} & A_{22}
\end{array}\right] \underline{x}(t)+\left[\begin{array}{c}
0_{p \times r} \\
B
\end{array}\right] \underline{u}(t)
$$

where the pair $\left\{A_{21}, B\right\}$ is completely controllable. The system (B-3) is stabilizable iff the matrix $A_{12}$ is asymptotically stable (all its eigenvalues have negative real parts).

The system (B-1) is a special case of (B-3). Because, the pair $\left\{\mathrm{A}_{2}, \underline{\mathrm{b}}_{2}\right\}$ is completely controllable (see Equation (5.31a), we can apply the above theorem. The system (B-1) will be stabilizable iff the matrix $A_{7}$ is stable. Therefore, we need to show that all the eigenvalues of $A_{7}$ have negative real parts in order to prove the stabilizability of the system (B-1).

$A_{7}$ defined in Equation (B-2) is a lower triangular matrix.

Hence, the set of its eigenvalues consists of the eigenvalues of the matrices $A_{6}$ and $A_{D 3}$. As discussed in chapter III, the closed-loop matrix $A_{6}$ defined in Equation (5.26) is stable. The matrix $A_{D 3}$ is also stable, since each of its main diagonal elements defined by Equation (5.28) is stable. Therefore, all the eigenvalues the two matrices $A_{6}$ and $A_{D 3}$ have negative real parts. This implies the stability of the matrix $\mathrm{A}_{7}$. 


\title{
APPENDIX C \\ SOLUTION OF A SIMPLE OPTIMAL \\ TRACKING PROBLEM
}

\begin{abstract}
In this appendix we present a detailed solution of the tracking problem discussed in section 6.2, where we observed that for a fixed plant parameter, the error, as a function of reference parameter, monotonically decreases and will reach a minimum at point where the reference parameter equals the plant parameter and then monotonically increases to reach a steady state value. This nonmonotonic trend of the rms tracking error will be shown theoretically in this appendix.
\end{abstract}

\section{C.1 The Problem}

Consider the simple optimal stochastic tracking problem illustrated in Figure C.1. The upper block diagram represents the reference model with external disturbance input $w$ and the lower block diagram represents the plant whose output tracks the output of the reference model. The plant is disturbed by the same external input w. Both reference model and plant are represented by first order systems with model parameters $\tau_{3}$ and $\tau_{2}$, respectively. For this system, we wish to find the steady state rms tracking error as a function of model parameters $\tau_{3}$ and $\tau_{2}$. As seen in Figure C.1, this 


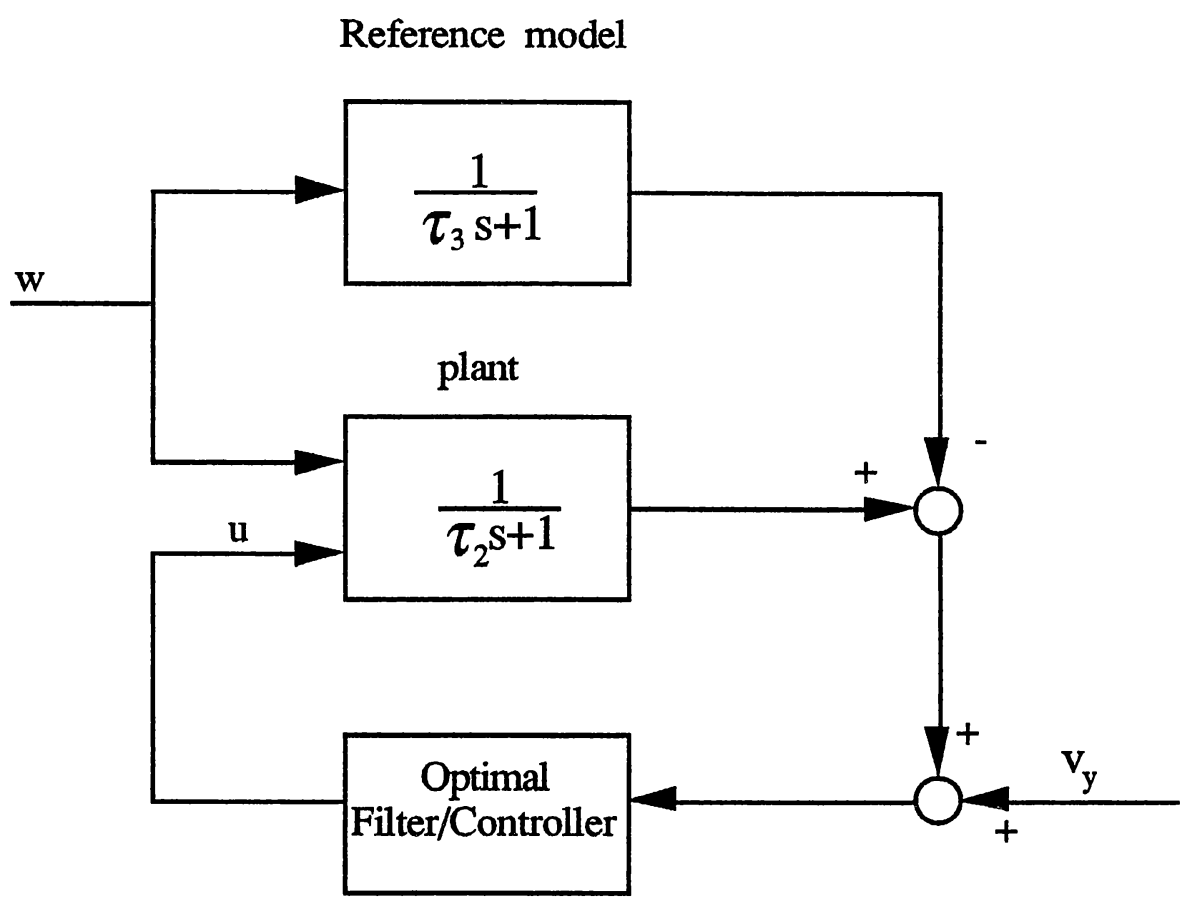

Figure C.1 A Simple Optimal Tracking Problem

\section{C.2 The Solution}

The reference model is defined by the following state equations

$$
\begin{aligned}
& \dot{\mathrm{x}}_{1}(\mathrm{t})=-\mathrm{ax}_{1}(\mathrm{t})+\mathrm{a} \mathrm{w}(\mathrm{t}) \\
& \mathrm{y}_{1}(\mathrm{t})=\mathrm{x}_{1}(\mathrm{t})
\end{aligned}
$$

and the plant is described by the state differential equations.

$$
\begin{aligned}
& \dot{x}_{2}(t)=-b x_{1}(t)+b u(t)+b w(t) \\
& y_{2}(t)=x_{2}(t)
\end{aligned}
$$

where 


$$
a=\frac{1}{\tau_{3}} \text { and } b=\frac{1}{\tau_{2}}
$$

and $w(t)$ is a Gaussian white noise with intensity $W$.

We wish the plant output $y_{2}$ to track the reference variable $y_{1}$ as closely as possible by choosing the input $u^{*}(t)$ which minimizes the performance index $\mathrm{J}$.

$$
\mathrm{J}=\mathrm{E}\left\{\left(\mathrm{y}_{2}-\mathrm{y}_{1}\right)^{2}+\mathrm{g} \mathrm{u}^{2}\right\}
$$

The plant equation and the reference model equation can be combined by defining the augmented state $x_{3}(t)$ which satisfies

$$
\underline{\dot{x}}_{3}(t)=A_{3} \underline{x}_{3}(t)+\underline{b}_{3} u(t)+\underline{e}_{3} w(t)
$$

with

$$
\underline{x}_{3}=\left[\begin{array}{l}
x_{1} \\
x_{2}
\end{array}\right], A_{3}=\left[\begin{array}{cc}
-a & 0 \\
0 & -b
\end{array}\right], \underline{b}_{3}=\left[\begin{array}{l}
0 \\
b
\end{array}\right], \underline{e}_{3}=\left[\begin{array}{l}
a \\
b
\end{array}\right], C_{3}=\left[\begin{array}{ll}
-1 & 1
\end{array}\right] .
$$

The observed variable for the augmented system is

$$
y(t)=y_{2}-y_{1}+v_{y}=C_{3} \underline{x}_{3}(t)+v_{y}(t)
$$

where $v_{y}(t)$ is a Gaussian white noise process with intensity $v_{y}$. In terms of $\underline{x}_{3}$, the performance index $\mathrm{J}$ becomes

$$
J=E\left\{\underline{x}_{3}^{\prime}(t) Q \underline{x}_{3}(t)+g u^{2}(t)\right\}
$$

with

$$
Q=\left[\begin{array}{cc}
1 & -1 \\
-1 & 1
\end{array}\right]
$$

Now, we have a linear stochastic optimal regulator problem with the augmented plant defined by Equations (C-4)-(C-5) and the index of 
performance J given in Equation (C-6). By referring to chapter III, we can determine the optimal linear controller as:

$$
u^{*}(t)=-F \underline{\hat{x}}_{3}(t)
$$

where the optimal controller gain $F$ is

$$
F=\frac{1}{g} \underline{b}_{3}^{\prime} P=\frac{1}{g}\left[\begin{array}{ll}
0 & b
\end{array}\right] P=\frac{b}{g}\left[\begin{array}{ll}
p_{12} & p_{22}
\end{array}\right]
$$

and the positive definite matrix $P$ is the steady state solution of the Riccati equation

$$
\mathrm{Q}-\frac{1}{\mathrm{~g}} \mathrm{P} \underline{\mathrm{b}}_{3} \underline{\mathrm{b}}_{3}^{\prime} \mathrm{P}+\mathrm{A}_{3}^{\prime} \mathrm{P}+\mathrm{PA}_{3}=0_{2 \times 2}
$$

The following nonlinear equations solve for $P$.

$$
\begin{aligned}
& 1-\frac{b^{2}}{g} p_{12}^{2}-2 a p_{11}=0 \\
& -1-\frac{b^{2}}{g} p_{12} p_{22}-(b+a) p_{12}=0 \\
& 1-\frac{b^{2}}{g} p_{22}^{2}-2 b p_{22}=0
\end{aligned}
$$

The last Equation in (B-10) solves for $\mathrm{p}_{22}$.

$$
p_{22}=\frac{1}{b}[-g \pm \sqrt{g(1+g)}]
$$

$\mathrm{p}_{22}$ must be positive, then

$$
\mathrm{p}_{22}=\frac{1}{\mathrm{~b}}[-\mathrm{g}+\sqrt{\mathrm{g}(1+\mathrm{g})}]
$$


As seen, $p_{22}$ is independent of reference model parameter $\tau_{3}$. It yields a feedback link of the optimal controller (see Equation C-8) which is independent of the reference model parameters. This property of tracking problems has been fully discussed in section 3.8. Using (C-11) in the first two Equations (C-10), we can solve for $\mathrm{p}_{11}$ and $\mathrm{P}_{12}$ as:

$$
\mathrm{p}_{12}=-\frac{1}{\gamma}, \quad \mathrm{p}_{11}=\frac{1}{2}\left[1-\frac{\mathrm{b}^{2}}{\mathrm{~g} \gamma^{2}}\right]
$$

with

$$
\gamma=(a+b)+\frac{b}{g}(-g+\sqrt{g(1+g)})
$$

By using (B-11)-(B-13) in (B-8), we can write optimal controller gains as:

$$
F=\left[\begin{array}{ll}
f_{1} & f_{2}
\end{array}\right]
$$

where

$$
f_{1}=-\frac{b}{g}\left(\frac{1}{a+b \sqrt{1+\frac{1}{g}}}\right), f_{2}=-1+\sqrt{1+\frac{1}{g}} .
$$

Figure C.2 illustrates the optimal tracking control of the augmented system $\underline{x}_{3}$ when we have perfect measurement. For the system depicted in Figure C.2, we wish to show that under what conditions the tracking error will be zero (perfect match). In other words, we need to check that when the the transfer function $x_{2} / w$ equals $\mathrm{x}_{1} / \mathrm{w}$. This can be done as follows. Let us denote 


$$
G=\frac{1}{\tau_{3} s+1} \text { and } H=\frac{1}{\tau_{2} s+1}
$$

Now, we can write the transfer functions $x_{2} / w$ and $x_{1} / w$ in terms of $G$, $F$, and $H$ as:

$$
\begin{aligned}
& \frac{x_{1}}{w}=G \\
& \frac{x_{2}}{w}=\frac{H\left(1-f_{1} G\right)}{1+H_{2}} .
\end{aligned}
$$

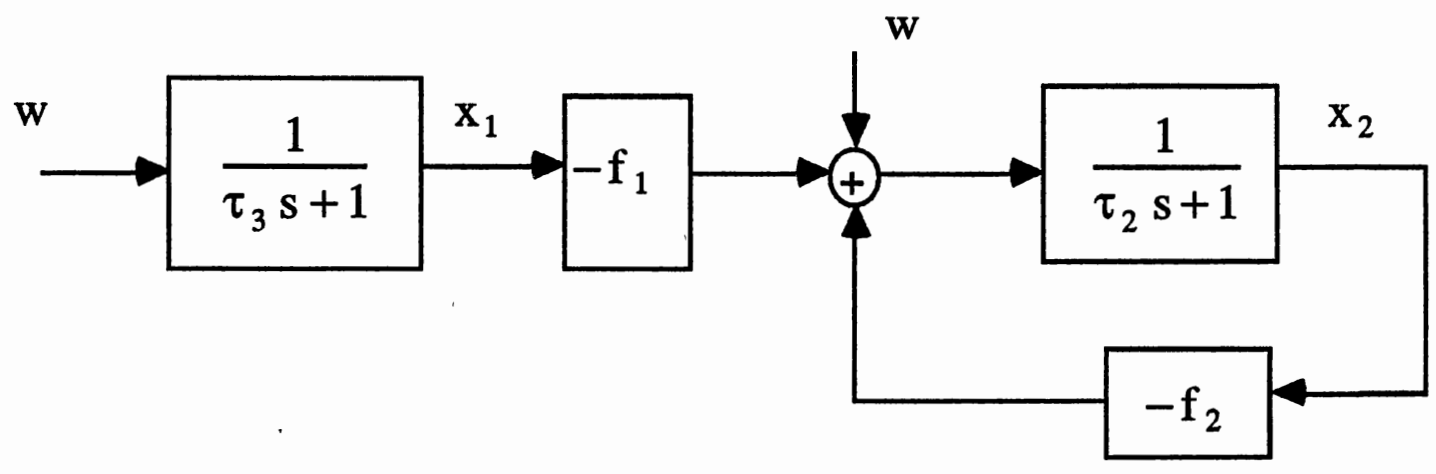

Figure C.2 Optimal Tracking Control of the System $\underline{x}_{3}$

By equating the right hand sides of the above two equations, we will have

$$
G\left(1+H f_{2}\right)=H\left(1-f_{1} G\right)
$$

or

$$
\frac{.1}{\tau_{3} s+1}\left(1+\frac{f_{2}}{\tau_{2} s+1}\right) \equiv \frac{1}{\tau_{2} s+1}\left(1-\frac{f_{1}}{\tau_{3} s+1}\right)
$$


By simplifying the above equation, we can have the following identity.

$$
\tau_{2} s+1+f_{2} \equiv \tau_{3} s+1-f_{1}
$$

This will be satisfied iff $\tau_{2}=\tau_{3} \& f_{1}+f_{2}=0$. From (C-14), we can see that if $\tau_{2}=\tau_{3}$ then we can write

$$
f_{1}=-\frac{b}{g}\left(\frac{1}{a+b \sqrt{1+\frac{1}{g}}}\right)=-\frac{1}{g}\left(\frac{1}{1+\sqrt{1+\frac{1}{g}}}\right)=-\frac{1}{g} \frac{1-\sqrt{1+\frac{1}{g}}}{1-1+\frac{1}{g}}=-f_{2} .
$$

Therefore, in order to have a zero rms tracking error, the plant parameter $\tau_{2}$ must be equal to reference model parameter $\tau_{3}$.

\section{C.2.1. Controller Poles:}

The controller poles are the eigenvalues of the closed loop matrix $A_{c}$.

$$
A_{c}=A_{3}-\underline{b}_{3} F=\left[\begin{array}{cc}
-a & 0 \\
-b f_{1} & -b\left(1+f_{2}\right)
\end{array}\right]
$$

$A_{c}$ is a diagonal matrix. Its eigenvalues are the main diagonal elements. Therefore, closed-loop poles will be $-a$ and $-b\left(1+f_{2}\right)$ or

Controller poles $=\left\{-\frac{1}{\tau_{3}},-\frac{\sqrt{1+\frac{1}{g}}}{\tau_{2}}\right\}$ 
C-8

\section{C.2.2 Observer Poles:}

The estimate of the augmented state $\underline{x}_{3}$ is determined by the following Kalman filter (see section 3.6):

$$
\underline{\hat{\mathbf{x}}}_{3}(t)=\mathrm{A}_{3} \underline{\hat{x}}_{3}(t)+\mathrm{B}_{3} \mathrm{u}(\mathrm{t})+\mathrm{KC} \mathrm{C}_{3}\left[\underline{\mathrm{x}}_{3}(\mathrm{t})-\underline{\hat{x}}_{3}(\mathrm{t})\right]
$$

where

$$
\mathrm{K}=\mathrm{RC}_{3}^{\prime} \mathrm{V}_{\mathrm{y}}^{-1}
$$

and the covariance positive definite symmetric matrix $R$ is the solution of the Ricaati Equation

$$
\mathrm{W}_{3}-\mathrm{RC}_{3}^{\prime} \mathrm{V}_{\mathrm{y}}^{-1} \mathrm{C}_{3} \mathrm{R}+\mathrm{A}_{3} \mathrm{R}+\mathrm{RA}_{3}^{\prime}=0
$$

with

$$
\mathrm{W}_{3}=\underline{\mathrm{e}}_{3} \mathrm{~W} \underline{\mathrm{e}}_{3}^{\prime}=\left[\begin{array}{ll}
\mathrm{a}^{2} & \mathrm{ab} \\
\mathrm{ba} & \mathrm{b}^{2}
\end{array}\right] \mathrm{W} .
$$

Let us denote $R=\left[\begin{array}{ll}r_{11} & r_{12} \\ r_{12} & r_{22}\end{array}\right]$.

By expanding (C-17), we will have the following equations which solve for $\mathbf{R}$.

$$
\begin{aligned}
& \mathrm{Wa} \mathrm{a}^{2}-\frac{1}{\mathrm{~V}_{\mathrm{y}}} \alpha^{2}-2 \mathrm{ar}_{11}=0 \\
& \mathrm{Wa} \mathrm{b}-\frac{1}{\mathrm{~V}_{\mathrm{y}}} \alpha \beta-(\mathrm{a}+\mathrm{b}) \mathrm{r}_{12}=0 \\
& \mathrm{~Wb}^{2}-\frac{1}{\mathrm{~V}_{\mathrm{y}}} \beta^{2}-2 \mathrm{br}_{22}=0
\end{aligned}
$$

where 


$$
\alpha=r_{11}-r_{12}, \beta=r_{12}-r_{22} .
$$

From (C-18b), we can write

$$
r_{12}=\frac{W a b V_{y}-\delta}{(a+b) V_{y}}
$$

where $\alpha \beta=\delta$.

By substituting (C-19) into (C-18a) and (C-18c), we can have the following nonlinear equations.

$$
\begin{aligned}
& \beta^{2}(a+b)-2 b(a+b) V_{y} \beta-W b^{2}(b-a) V_{y}-2 \delta=0 \\
& \alpha^{2}(a+b)+2 a(a+b) V_{y} \alpha+W a^{2}(b-a) V_{y}-2 a \delta=0
\end{aligned}
$$

Equations (C-20) solve for $\alpha$ and $\beta$. Knowing these two values, we can use (C-18d) and (C-19) to determine R. It can be shown that the following fourth order equation solves for $\beta$.

$$
\begin{aligned}
& {\left[-(a+b)^{3}+4 a b(a+b)\right] \beta^{4}+} \\
& {\left[4 a b(a+b)^{2} V_{y}+4 b(a+b)^{3} V_{y}-8 b^{2} a(a+b) V_{y}\right] \beta^{3}+} \\
& {\left[-4 b^{2} a^{2} W(b-a) V_{y}-4 b^{2}\left(a+b^{3}\right) V_{y}^{2}+2(a+b)^{2} b^{2} W(b-a) V_{y}-\right.} \\
& \left.8 b^{2} a(a+b)^{2} V_{y}^{2}-4 b^{3} a(b-a) W V_{y}\right] \beta^{2}+ \\
& -4 b^{3}\left(b^{2}-a^{2}\right) V_{y}^{2}[a+(a+b) W] \beta+ \\
& -b^{4} W^{2}(b-a)^{2}(a+b) V_{y}^{2}=0
\end{aligned}
$$

As seen, we should deal with highly nonlinear equations in order to determine $R$. Let us determine the corresponding values of $R$ for the following interesting values of reference parameter, $\tau_{3}$. 
i. $\mathrm{R}^{0}=\lim _{\tau_{3} \rightarrow 0} \mathrm{R}$

It can be shown that we can obtain the following results.

$$
\begin{aligned}
& \alpha^{0}=\lim _{\tau_{3} \rightarrow 0} \alpha=a_{y}\left(-1+\sqrt{1+\frac{W}{V_{y}}}\right) \\
& \beta^{0}=\lim _{\tau_{3} \rightarrow 0} \beta=b V_{y}\left(-1+\sqrt{1+\frac{W}{V_{y}}}\right)
\end{aligned}
$$

Knowing that $\tau_{3} \rightarrow 0 \Rightarrow a \rightarrow \infty$, from (C-18d) and (C-22) we will have

$$
\mathrm{R}^{0}=\left[\begin{array}{ll}
\infty & \mathbf{r}_{12}^{0} \\
\mathbf{r}_{12}^{0} & \mathbf{r}_{22}^{0}
\end{array}\right]
$$

where

$$
\begin{aligned}
& \mathrm{r}_{12}^{0}=\lim _{\tau_{3} \rightarrow 0} \mathrm{r}_{12}=\mathrm{Wb}-\beta^{0}\left(-1+\sqrt{1+\frac{\mathrm{W}}{\mathrm{V}_{\mathrm{y}}}}\right) \\
& \mathrm{r}_{22}^{0}=\lim _{\tau_{3} \rightarrow 0} \mathrm{r}_{22}=\mathrm{Wb}-\beta^{0} \sqrt{1+\frac{\mathrm{W}}{\mathrm{V}_{\mathrm{y}}}}
\end{aligned}
$$

ii. $\tau_{3}=\tau_{2} \Rightarrow a=b$

For this case the covariance matrix $\mathrm{R}$ becomes

$$
\begin{aligned}
& \mathrm{R}_{\mathrm{a}=\mathrm{b}}=\frac{\mathrm{Wa}}{2}\left[\begin{array}{ll}
1 & 1 \\
1 & 1
\end{array}\right] . \\
& \alpha^{\mathrm{a}=\mathrm{b}}=\beta^{\mathrm{a}=\mathrm{b}}=0 .
\end{aligned}
$$


iii. $\tau_{3} \rightarrow \infty \Rightarrow \mathrm{a} \rightarrow 0$

For this case, we can have the following error covariance matrix.

$$
\bar{R}=\lim _{\tau_{3} \rightarrow \infty} R=\left[\begin{array}{cc}
0 & 0 \\
0 & -\bar{\beta}
\end{array}\right]
$$

where

$$
\bar{\beta}=b V_{y}\left(1-\sqrt{1+\frac{W}{V_{y}}}\right)
$$

The observer gain is calculated as:

$$
\mathrm{K}=\frac{1}{\mathrm{~V}_{\mathrm{y}}} \mathrm{RC}_{3}^{\prime}=\frac{1}{\mathrm{~V}_{\mathrm{y}}}\left[\begin{array}{ll}
\mathrm{r}_{11} & \mathrm{r}_{12} \\
\mathrm{r}_{12} & \mathrm{r}_{22}
\end{array}\right]\left[\begin{array}{c}
-1 \\
1
\end{array}\right]=-\frac{1}{\mathrm{~V}_{\mathrm{y}}}\left[\begin{array}{l}
\alpha \\
\beta
\end{array}\right]
$$

The observer poles are the eigenvalues of the matrix

$$
A^{0}=A_{3}-K C_{3}=\left[\begin{array}{cc}
-a-\frac{\alpha}{V_{y}} & \frac{\alpha}{V_{y}} \\
\frac{-\beta}{V_{y}} & -b+\frac{\beta}{V_{y}}
\end{array}\right]
$$

The eigenvalues of the observer matrix $\mathrm{A}^{0}$ are the roots of characteristic polynomial

$$
\lambda^{2}+\left(a+b+\frac{\alpha-\beta}{V_{y}}\right) \lambda+a b+\frac{b \alpha-\alpha \beta}{V_{y}}-\frac{2 \alpha \beta}{V_{y}^{2}}=0
$$


Now, let us determine the observer gains and observer poles for the cases mentioned before.

$$
\begin{aligned}
& \mathrm{K}_{0}=\lim _{\tau_{3} \rightarrow 0} \mathrm{~K}=\frac{1}{\mathrm{~V}_{\mathrm{y}}}\left[\begin{array}{l}
-\alpha^{0} \\
-\beta^{0}
\end{array}\right] \\
& \mathrm{K}_{\mathrm{a}=\mathrm{b}}=\frac{1}{\mathrm{~V}_{\mathrm{y}}}\left[\begin{array}{l}
0 \\
0
\end{array}\right]=\left[\begin{array}{l}
0 \\
0
\end{array}\right] \\
& \overline{\mathrm{K}}=\lim _{\tau_{3} \rightarrow \infty}=-\frac{1}{\mathrm{~V}_{\mathrm{y}}}\left[\begin{array}{l}
0 \\
\bar{\beta}
\end{array}\right]
\end{aligned}
$$

From Equations (C-27), we can see that for small $\tau_{3}$ one of observer poles occurs on the left half of s-plane at a point far from the imaginary axis corresponding to $\mathrm{K}_{0}(2)=-\alpha_{0} / \mathrm{V}_{\mathrm{y}}$ which is a big negative number and the other pole occurs at a point on the left which is closer to the imaginary axis because of $\mathrm{K}_{0}(2)=-\beta_{0} / \mathrm{V}_{\mathrm{y}}$. For $\tau_{3}=\tau_{2}$, both observer poles occur at $-1 / \tau_{2}$. As $\tau_{3}$ approaches infinity one pole goes to origin and the other pole approaches $-b+\bar{\beta} / V_{y}$.

\section{C.2.3 Evaluation of the Performance Index}

The following dynamical equation describes the reconstruction error $\underline{e}(t)=\underline{x}_{3}(t)-\underline{\hat{x}}_{3}(t)$.

$$
\underline{\dot{e}}(t)=A^{0} \underline{e}(t)+\underline{e}_{3} \underline{w}_{3}(t)-K v_{y}(t)
$$

The estimator can be written in terms of $\underline{e}(t)$ as:

$$
\underline{\hat{x}}_{3}(t)=K C_{3} \underline{e}(t)-\left(A_{3}-\underline{b}_{3} F\right) \underline{\hat{x}}_{3}(t)+K v_{y}(t)
$$


By combining equations (C-28a)and (C-28b), we will have

$$
\begin{aligned}
{\left[\begin{array}{c}
\dot{\dot{e}}(t) \\
\underline{\hat{x}}_{3}(t)
\end{array}\right] } & =\left[\begin{array}{cc}
A_{3}-K^{0} C_{3} & 0_{2 \times 2} \\
K^{0} C_{3} & A_{3}-\underline{b}_{3} F
\end{array}\right]\left[\begin{array}{c}
\underline{e}(t) \\
\underline{\hat{x}}_{3}(t)
\end{array}\right] \\
& +\left[\begin{array}{cc}
\underline{e}_{3} & -K \\
0_{2 \times 1} & K
\end{array}\right]\left[\begin{array}{l}
\underline{w}_{3}(t) \\
v_{y}(t)
\end{array}\right] .
\end{aligned}
$$

The covariance matrix of the above augmented vector can be found and all mean square of interest can be calculated from this covariance matrix. Since $\underline{\hat{x}}_{3}$ and $\underline{\mathrm{e}}$ are uncorrelated regardless of input of $u(t)$, we may denote the covariance matrix of the above augmented vector as

$$
E\left\{\left[\begin{array}{c}
\underline{\mathrm{e}}(\mathrm{t}) \\
\underline{\hat{\mathrm{x}}}_{3}(\mathrm{t})
\end{array}\right]\left[\underline{\mathrm{e}}(\mathrm{t}) \quad \underline{\hat{x}}_{3}(\mathrm{t})\right]^{\prime}\right\}=\left[\begin{array}{cc}
\mathrm{R} & 0_{2 \times 2} \\
0_{2 \times 2} & \gamma
\end{array}\right] \text {. }
$$

where $\gamma=E\left[\underline{\hat{x}}_{3}(t) \underline{\hat{x}}_{3}^{\prime}(t)\right]$ and it can be determined as the steady state solution of the matrix equation (see Theorem 1.52 of [67])

$$
\overline{\mathrm{A}} \gamma+\gamma \overline{\mathrm{A}}^{\prime}+\mathrm{KV}_{\mathrm{y}} \mathrm{K}^{\prime}=0_{2 \times 2}
$$

where

$$
\bar{A}=A_{3}-\underline{b}_{3} F==\left[\begin{array}{cc}
-a & 0 \\
-b f_{1} & -b\left(1+f_{2}\right)
\end{array}\right] .
$$

By letting

$$
\gamma=\left[\begin{array}{ll}
\gamma_{11} & \gamma_{12} \\
\gamma_{12} & \gamma_{22}
\end{array}\right]
$$


and using Equations (C-25) and (C-32) in Equation (C-31), the following equations solve for $\gamma$.

$$
\begin{aligned}
& -2 a \gamma_{11}+\frac{\alpha^{2}}{V_{y}}=0 \\
& b f_{1} \gamma_{11}+\gamma_{12}\left(a+b\left(1+f_{2}\right)\right)-\frac{\alpha \beta}{V_{y}}=0 \\
& 2 b_{1} \gamma_{12}+2 b\left(1+f_{2}\right) \gamma_{22}-\frac{\beta^{2}}{V_{y}}=0
\end{aligned}
$$

Equations (C-33) yield

$$
\begin{aligned}
& \gamma_{11}=\frac{\alpha^{2}}{2 a V_{y}}, \gamma_{12}=\frac{2 a \alpha \beta-b f_{1} \alpha^{2}}{2 a\left(a+b \sqrt{1+\frac{1}{g}}\right) V_{y}} \\
& \gamma_{22}=\frac{2 a \beta^{2}\left(a+b \sqrt{1+\frac{1}{g}}\right)-2 b f_{1}\left(2 a \alpha \beta-b f_{1} \alpha^{2}\right)}{4 a b V_{y}\left(a b \sqrt{1+\frac{1}{g}}+b^{2}\left(1+\frac{1}{g}\right)\right)} .
\end{aligned}
$$

To obtain Equations (C-34), we used the fact that $1+\mathrm{f}_{2}=\sqrt{1+\frac{1}{g}}$ (see Equation (C-14)). Let us determine $\gamma$ for the following cases.

$$
\text { i. } \quad \tau_{3} \rightarrow 0
$$

As $\tau_{3}$ approaches zero both $a$ and $\alpha$ approach infinity, $f_{1}$ goes to 0 , and $\beta$ approaches $\beta^{0}$. Using $(C-22)$, then we can 
write

$$
\begin{aligned}
& \gamma_{11}^{0}=\lim _{\tau_{3} \rightarrow 0} \gamma_{11}=\infty \\
& \gamma_{12}^{0}=\lim _{\tau_{3} \rightarrow 0} \gamma_{12} \cong \frac{\beta^{0}}{V_{y}} \cong b\left(1-\sqrt{1+\frac{W}{V_{y}}}\right) \\
& \gamma_{22}^{0}=\lim _{\tau_{3} \rightarrow 0} \gamma_{22} \cong \frac{\left(\beta^{0}\right)^{2}}{4 b^{2} V_{y} \sqrt{1+\frac{1}{g}}} \cong \frac{V_{y}\left(1-\sqrt{1+\frac{W}{V_{y}}}\right)^{2}}{4 \sqrt{1+\frac{1}{g}}} .
\end{aligned}
$$

ii. $\tau_{3}=\tau_{2}$.

This implies that $a=b$ which in turn yields (see Equation (C-14))

$$
\mathrm{f}_{1}=-\mathrm{f}_{2}=1-\sqrt{1+\frac{1}{\mathrm{~g}}} .
$$

For $a=b$, the observer gain is zero (see Equation (C-27b)). Therefore, we will have

$$
\gamma_{\mathrm{a}=\mathrm{b}}=0_{2 \times 2} \text {. }
$$

iii. $\quad \tau_{3} \rightarrow \infty$.

For this case, "a" goes to zero, $\beta$ approaches $\bar{\beta}$, and $\alpha$ tends to 0 . Then, we can write

$$
\bar{\gamma}_{11}=\lim _{\tau_{3} \rightarrow \infty} \gamma_{11}=0, \bar{\gamma}_{12}=\lim _{\tau_{3} \rightarrow \infty} \gamma_{12}=0
$$




$$
\bar{\gamma}_{22}=\lim _{\tau_{3} \rightarrow \infty} \gamma_{22} \cong \frac{\bar{\beta}^{2}}{2 b^{2} V_{y} \sqrt{1+\frac{1}{g}}} \cong \frac{V_{y}\left(1-\sqrt{1+\frac{W}{V_{y}}}\right)^{2}}{2 \sqrt{1+\frac{1}{g}}}
$$

\section{C.2.4 Evaluating of the Mean Square Error, $\mathrm{E}_{\mathrm{r}}$.}

Knowing that $\underline{e}(t)$ and $\underline{\hat{x}}_{3}(t)$ are uncorrelated and $\underline{\mathrm{x}}_{3}(\mathrm{t})=\underline{\mathrm{e}}(\mathrm{t})+\underline{\hat{x}}_{3}(\mathrm{t})$, the covariance of $\underline{\mathrm{x}}_{3}(\mathrm{t})$ is

$$
\mathrm{E}\left[\underline{\mathrm{x}}_{3} \underline{\underline{\mathrm{x}}}_{3}^{\prime}\right]=\mathrm{E}\left[\underline{\mathrm{e}}_{3} \underline{\mathrm{e}}_{3}^{\prime}\right]+\mathrm{E}\left[\underline{\underline{\hat{x}}}_{3} \underline{\hat{x}}_{3}^{\prime}\right]=\mathrm{R}+\gamma .
$$

$E_{r}$ is found as

$$
\begin{aligned}
E_{r} & =C_{3}(R+\gamma) C_{3}^{\prime}=\left[\begin{array}{ll}
1 & -1
\end{array}\right]\left[\begin{array}{ll}
r_{11}+\gamma_{11} & r_{12}+\gamma_{12} \\
r_{12}+\gamma_{12} & r_{22}+\gamma_{22}
\end{array}\right]\left[\begin{array}{c}
1 \\
-1
\end{array}\right]= \\
& =r_{11}-r_{12}+r_{22}-r_{12}-2 \gamma_{12}+\gamma_{22}+\gamma_{11}= \\
& =\alpha-\beta-2 \gamma_{12}+\gamma_{22}+\gamma_{11} .
\end{aligned} .
$$

Let us evaluate the mean squared error for the following cases.

i. $\quad \tau_{3} \rightarrow 0$

For this case, using (C-22) and (C-35), Equation (C-38) becomes

$$
E_{r}^{0}=\lim _{\tau_{3} \rightarrow 0} E_{r}=\alpha^{0}-\beta^{0}-2 \gamma_{12}^{0}+\gamma_{11}^{0}+\gamma_{22}^{0} \cong \infty
$$


ii. $\tau_{3}=\tau_{2}$.

For this case, the mean square error is zero; use (C-23) and $(\mathrm{C}-36)$ in $(\mathrm{C}-39)$.

$$
E_{r}^{a=b}=0
$$

iii. $\quad \tau_{3} \rightarrow \infty$.

Using (C-24) and (C-27) in (C-39), the error becomes

$$
\begin{aligned}
\overline{\mathrm{E}}_{\mathrm{r}} & =\lim _{\tau_{3} \rightarrow \infty} \mathrm{E}_{\mathrm{r}}=\bar{\alpha}-\bar{\beta}-2 \bar{\gamma}_{12}+\bar{\gamma}_{11}+\bar{\gamma}_{22} \\
& =-\bar{\beta}+\frac{\bar{\beta}^{2}}{2 \mathrm{~b}^{2} \mathrm{~V}_{\mathrm{y}} \sqrt{1+\frac{1}{\mathrm{~g}}}}= \\
& =\frac{\mathrm{V}_{\mathrm{y}}\left(1-\sqrt{1+\frac{\mathrm{W}}{\mathrm{V}_{\mathrm{y}}}}\right)}{2 \sqrt{1+\frac{1}{\mathrm{~g}}}}\left(1-\sqrt{1+\frac{\mathrm{W}}{\mathrm{V}_{\mathrm{y}}}}-2 \mathrm{~b} \sqrt{1+\frac{1}{\mathrm{~g}}}\right) .
\end{aligned}
$$

From Equation (C-41), we can list the following two properties for $\overline{\mathrm{E}}_{\mathrm{r}}$.

1. $\overline{\mathrm{E}}_{\mathrm{r}}\left(\tau_{2}\right)$ is always positive because $\bar{\beta}$ is negative (see Equation $(C-24 b))$.

2. As $\tau_{2}$ increases, $\overline{\mathrm{E}}_{\mathrm{r}}\left(\tau_{2}\right)$ will decrease.

Therefore, for a fixed plant parameter $\tau_{2}$ the mean square error, $E_{r}$, as a function of $\tau_{3}$ is expected to have the shape of response 
depicted in Figure 6.9. It starts with a big positive number (when $\tau_{3}$ is very small) and decreases monotonically till becomes zero at $\tau_{3}=\tau_{2}$ before increasing and reaching its steady state value, $\overline{\mathrm{E}}_{\mathrm{r}}$. This value is dependent only on $\tau_{2}$ and will decrease as $\tau_{2}$ increases. 


\section{VITA \\ Mohammad B. Menhaj \\ Candidate for the Degree of \\ Doctor of Philosophy}

Thesis: ANALYSIS OF DELAYS IN NETWORKED FLIGHT SIMULATORS

MAJOR FIELD: Electrical Engineering

Biographical:

Personal Data: Born in Rasht, Iran, April 24, 1959, the son of Mr. Jamshid Menhaj and Mrs. Maryam Azizian.

Education: Graduated From Melli University, Tehran Iran, in June 1981; received Master of Science degree in Electrical Engineering from Oklahoma State University in Summer 1988; completed requirement for the Doctor of Philosophy degree at Oklahoma State University in May 1992.

Professional Experience: Computer System Designer, Depatment of Computer in Ministry of Power, Tehran Iran, 1984-1986; Graduate Teaching and Research Assistant, Department of Electrical and Computer Engineering, Oklahoma State University, 1987-1992.

Professional Organizations: Member of the Institute of Electrical and Electronic Engineers and Tau Beta Phi. 